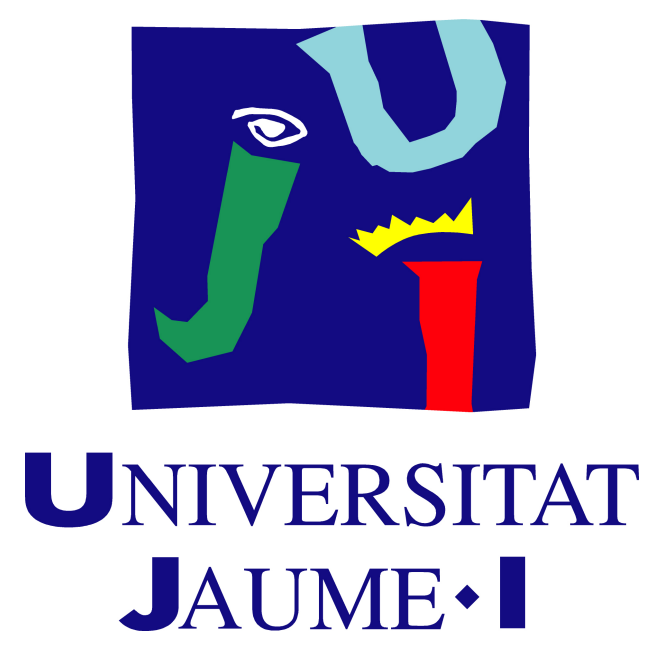

Apego, neurocognición y cognición
social en el espectro de la esquizofrenia

Doctoranda: Cristina Monfort Escrig

Director: Josep Pena Garijo

Enero 2021 



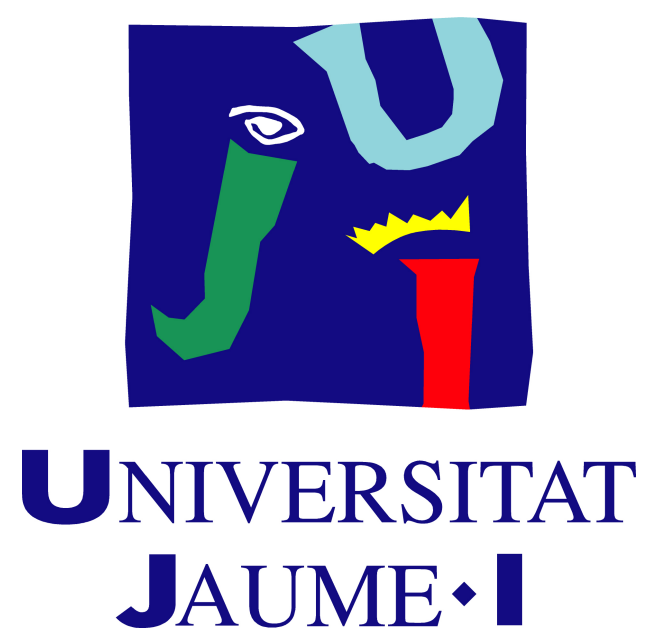

Programa de Doctorado en Psicología

Escuela de Doctorado de la Universitat Jaume I

Título de la tesis

Apego, neurocognición y cognición social en el espectro de la equizofrenia

Memoria presentada por Cristina Monfort Escrig para optar al grado de doctora por la Universitat Jaume I

Cristina Monfort Escrig

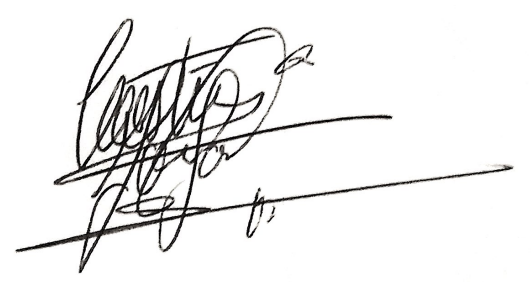

Josep Pena Garijo

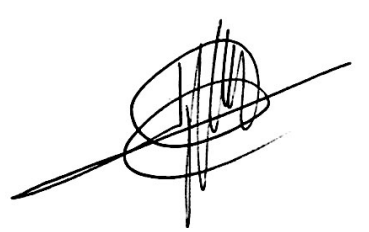

Castelló de la Plana, Enero 2021 
A mis padres, 


\section{Agradecimientos}

Desarrollar una tesis doctoral es un proceso largo y costoso, un camino angosto lleno de curvas, depresiones y cuestas, que exige mucha dedicación y competencia. Emprender un proyecto de este calibre no es fácil por todo lo que se deja en intermitencia, y por todo lo que se proyecta a un futuro de incerteza. La ambiciosa meta, a la que se dedica mucho tiempo vital, se visualiza en muchas ocasiones como lejana y difusa. Así que esta tesis no hubiera sido posible sin el apoyo de un sinfín de personas, que directa o indirectamente, han puesto su granito de arena para infundirme el ánimo necesario para persistir en este trabajo sin caer en un estado de desaliento.

En primer lugar, quisiera agradecer a mi director, el Dr. Josep Pena Garijo, por su enorme esfuerzo y constancia. Su entusiasmo por esta investigación, sumado a su gran profesionalidad y constante actualización en el campo, han sido un valioso incentivo para acometer este proyecto con la confianza necesaria. Sé que ha dedicado muchas horas al estudio y reflexión de cómo conseguir una íntegra y completa investigación, así como muchas horas a la orientación, revisión y actualización de contenidos. Pero sin duda, siento que le debo un profundo agradecimiento por haber sabido guiarme de la mejor manera posible, inspirándome confianza en mis propias capacidades.

Gracias a la Dra. María Ángeles Ruipérez, por aquella interesante conversación después de haber terminado mi defensa del Trabajo Final de Máster. Aquella charla inicial me permitió visualizar nuevas perspectivas y me encauzó a continuar en el ámbito de la investigación. Gracias a la Dra. Silvia Edo, por su amabilidad, cercanía y disponibilidad. Gracias también al Dr. David Gil, a la Dra. Marta Arrieta y a todo el equipo del Centro de Rehabilitación Psicosocial de Santander por su gentil acogimiento. 
Otro agradecimiento a mi grupo íntimo de amistades, que en esas veladas que se alargan sin una darse cuenta, me aportan esa sensación de comodidad, relajación y felicidad tan importantes para el día a día.

Especialmente, a mis padres, Juan y Piedad, porque desde su humildad y sencillez, siempre han respaldado mis decisiones y me han ayudado en todo cuanto han podido. A mis hermanos, Juan Carlos y David; a mi otra familia, Tere y Enrique, Xavi y Itziar; por su honestidad y afecto. Y, por supuesto, a mi compañero de viaje, Quico, porque el camino es más agradable y placentero cuando se comparte una vida de intereses y pasiones, ilusiones y sueños, logros y fracasos, desde el profundo y sincero cariño.

Por último, a todas aquellas personas anónimas que han participado en este estudio, a las cuáles quiero honestamente agradecer su aportación, así como el interés mostrado en los problemas de salud mental. Sin duda, la participación en cualquier tipo de investigación promueve el enriquecimiento de los recursos y las prácticas para mejorar la calidad de vida de las personas que los padecen.

En Castellón de la Plana, enero de 2021. 


\section{Resumen}

INTRODUCCIÓN: Las personas con trastornos del espectro de la esquizofrenia muestran déficits sustanciales en la neurocognición y la cognición social, así como problemas relacionados con el apego. El estudio de estos constructos parece clave para comprender la vulnerabilidad, el curso, el mantenimiento, la gravedad y el funcionamiento social en estas personas.

OBJETIVO: El objetivo general de esta tesis es el de explorar cómo estas variables se relacionan entre sí y cómo influyen en la sintomatología psicótica y en el funcionamiento social.

MÉTODO: Se diseñaron varios estudios para:

- Comprobar la adecuación del modelo reformulado de la Indefensión Aprendida para predecir los síntomas depresivos, y el funcionamiento social comparando un grupo de personas con trastornos del espectro de la esquizofrenia con otro de población sana.

- Explorar si una medida dimensional de apego diferenciaba entre ambos grupos y si algunas de estas dimensiones predecían distintas áreas del funcionamiento social dentro del grupo clínico.

- Generar un modelo de red interactivo entre los síntomas psicóticos, las dimensiones del apego y diferentes dominios cognitivos.

- Estudiar las diferencias en la gravedad de los déficits a través de un gradiente entre la esquizofrenia estabilizada, la psicosis temprana y la población normal.

- Proponer un modelo con las posibles vías en la que las variables de interés influían en el funcionamiento social.

Se utilizaron medidas de evaluación estandarizadas. Se analizaron los datos mediante pruebas clásicas como correlaciones bivariadas, comparaciones entre grupos (pruebas $t$, ANOVA) o regresiones lineales. Se probaron también métodos 
relativamente novedosos en el estudio de la psicopatología, como los análisis de redes y de mediación para estudiar las asociaciones entre las variables descritas. RESULTADOS: El modelo de la Indefensión resultó adecuado para predecir síntomas depresivos. Algunos estilos de atribución diferenciaron ambos grupos con grandes tamaños de efecto y predijeron algunos ámbitos del funcionamiento social en el grupo clínico. Además, se encontraron perfiles diferenciales en las dimensiones del apego, con un mayor apego inseguro (con predominio del estilo evitativo) en el grupo de personas con psicosis. Más allá de estos resultados, algunas dimensiones predijeron el funcionamiento social, independientemente de la gravedad de los síntomas. Se aporta evidencia preliminar sobre un modelo de red en el que el apego seguro resultó ser el nodo más central dentro de una red interactiva entre los síntomas, la cognición y las dimensiones del apego. Finalmente, los síntomas psicóticos mediaron la relación entre la "teoría de la mente" y el funcionamiento social, mientras que la neurocognición tuvo una influencia directa sobre dicho funcionamiento, independientemente de los síntomas.

CONCLUSIONES: Las personas con psicosis sufren importantes déficits en varios dominios de la cognición (básica y social) y exhiben un apego adulto inseguro. Dichas condiciones influyen significativamente en su funcionamiento social. La presente tesis ofrece evidencia preliminar acerca de la forma en que estas dimensiones se relacionan entre ellas y cómo influyen en el funcionamiento social de las personas con trastornos del espectro de la esquizofrenia. Se discuten las implicaciones teóricas y clínicas de estos hallazgos. 


\section{Abstract}

INTRODUCTION: People with schizophrenia spectrum disorders show substantial deficits in neurocognition, cognitive domains, social cognition, and attachment-related problems. The study of these constructs could be a key to understand the vulnerability, course, development and severity of the disease.

OBJECTIVE: The primary aim of this paper is to explore how these variables are related to each other and how they influence psychotic symptoms and social functioning.

METHOD: Several studies were designed to:

- Verify the adequacy of reformulated Learned Helplessness Model to predict depressive symptoms and social functioning by comparing a group of people with schizophrenia spectrum disorders with another group of healthy population.

- Explore if a dimensional measure of attachment differentiated between the two groups, and if some of these dimensions predicted different areas of social functioning within the clinical group.

- Generate an interactive network model between psychotic symptoms, attachment dimensions, and different cognitive domains.

- Study the differences in the severity deficits through a gradient between stabilized schizophrenia, early psychosis and the healthy population.

- Propose a model with the possible ways in which the variables of interest influenced social functioning.

Standardized evaluation measures were used. The data were analyzed using classical measures such as bivariate correlations, comparisons between groups ( $t$-tests, ANOVA) or linear regressions. Relatively novel methods in the study of psychopathology were also tested, such as network and mediation analyzes to study the associations between the described variables. 
RESULTS: The Helplessness model was adequate to predict depressive symptoms. Some attribution styles differentiated the two groups with large effect sizes and predicted some domains of social functioning in the clinical group. Furthermore, differential profiles were found in the attachment dimensions, with greater insecure attachment (with a predominance avoidance style) in the psychotic group. Beyond these results, some dimensions predicted social functioning, regardless of the symptoms severity. Preliminary evidence is provided on a network model in which secure attachment turned out to be the most central node within an interactive network between symptoms, cognition, and attachment dimensions. Finally, psychotic symptoms mediated the relationship between theory of mind and social functioning, while neurocognition had a direct influence on such functioning, regardless of symptoms.

CONCLUSIONS: People with psychosis suffer significant deficits in different domains of cognition (basic and social) and exhibit an adult insecure attachment. These conditions significantly influence their social functioning. This work offers preliminary evidence about the way in which these dimensions are related to each other and how they influence the social functioning of people with schizophrenia spectrum disorders. The theoretical and clinical implications of these findings are discussed. 


\section{Índice}

Agradecimientos

Resumen

Abstract

MARCO TEÓRICO

Introducción

Capítulo 1. Espectro de la esquizofrenia

1.1. Introducción

1.2. Clasificaciones diagnósticas

1.2.1. Clasificación diagnóstica DSM-5

1.2.2. Clasificación diagnóstica CIE-11 31

1.2.3. Comparación entre clasificaciones

1.3. Evaluación 37

1.4. Plan individualizado y adherencia al tratamiento 38

1.5. Intervención 39

1.5.1. Intervención farmacológica 39

1.5.2. Intervenciones psicosociales 41

Capítulo 2. Apego y psicosis

2.1. Introducción 43

2.2. Modelos teóricos del apego 44

2.2.1. Teoría del apego $\quad 44$

2.2.2. Modelos de trabajo $\quad 44$

2.3. Evaluación de los estilos de apego 47

2.4. Relaciones del apego con el espectro de las psicosis 49 
Capítulo 3. La cognición en la esquizofrenia (I): Concepto, métodos de evaluación y correlatos neurales

3.1. Introducción 51

3.2. La neurocognición en la esquizofrenia $\quad 52$

3.2.1. Evaluación $\quad 53$

3.2.2. Cerebro y neurocognición $\quad 55$

3.3. La cognición social en la esquizofrenia $\quad 59$

3.3.1 Procesos de cognición social en la esquizofrenia $\quad 60$

3.3.2. Evaluación de la cognición social 62

3.3.3. Bases neuroanatómicas asociadas a los procesos 63 sociales

Capítulo 4. La cognición en la esquizofrenia (II): Sesgos cognitivos, modelos explicativos y programas de intervención

4.1. Introducción 67

4.2. Sesgos cognitivos y sintomatología en la esquizofrenia $\quad 68$

4.2.1. Sesgos de razonamiento en las psicosis 68

4.2.2. Neurocognición y sesgo de salto a conclusiones 72

4.3. Relaciones entre neurocognición, cognición social, metacognición y funcionamiento social $\quad 73$

4.3.1. Modelos explicativos 73

4.3.2. Cognición y funcionamiento social 76

4.4. Programas de intervención $\quad 80$

4.4.1. Intervenciones sobre cognición $\quad 80$

4.4.2. Intervención sobre sesgos cognitivos 82

4.4.3. Correlatos neurales de las intervenciones sobre 83 cognición 
MARCO EXPERIMENTAL

Objetivos e hipótesis de la investigación

Descripción de los objetivos e hipótesis 87

Metodología $\quad 91$

Participantes y procedimiento $\quad 91$

Instrumentos de evaluación $\quad 92$

Análisis de datos $\quad 96$

Otras consideraciones $\quad 97$

Capítulo 5:

Estudio 1. Los estilos atribucionales y el funcionamiento

psicosocial en la esquizofrenia

$\begin{array}{ll}\text { Resumen } & 99\end{array}$

$\begin{array}{ll}\text { Artículo } & 100\end{array}$

Capítulo 6:

Estudio 2. Las dimensiones del apego como predictores del funcionamiento social en la esquizofrenia

$\begin{array}{ll}\text { Resumen } & 123\end{array}$

$\begin{array}{ll}\text { Artículo } & 124\end{array}$

Capítulo 7:

Estudio 3. La centralidad del apego seguro en una red interactiva entre los síntomas psicóticos, la cognición y las dimensiones del apego

Resumen

Artículo 
Capítulo 8:

Estudio 4. Predictores del funcionamiento social en personas con esquizofrenia

Resumen

Artículo

Discusión General

Principales hallazgos

Fortalezas

Limitaciones

Perspectivas de futuro

Conclusiones

Referencias Bibliográficas

ANEXOS

Anexo 1. Hoja informativa

Anexo 2. Hoja de consentimiento informado

Anexo 3. Hoja de datos

Anexo 4. PANSS

Anexo 5. PDI-21

Anexo 6. BDI-II

Anexo 7. SFS-R

Anexo 8. CAMIR-R

Anexo 9. ASQ

Anexo 10. Test de las Insinuaciones

Anexo 11. PERE

Anexo 12. Beads Task

Anexo 13. SCIP-S 


\section{Índice de tablas y figuras}

MARCO TEÓRICO

Capítulo 1. Espectro de la esquizofrenia

Tabla 1. Clasificación DSM-5 para los trastornos psicóticos

Tabla 2. DSM-5: Criterios diagnósticos para la esquizofrenia

Tabla 3. Clasificación CIE-11 para los trastornos psicóticos

Tabla 4. Codificación CIE-11 para la esquizofrenia

Capítulo 2. Apego y psicosis

Figura 1. Modelo de Bartholomew y Horowitz

Capítulo 3. La cognición en la esquizofrenia (I)

Tabla 5. Instrumentos de evaluación (MATRICS)

Tabla 6. Dominios de la cognición social

Figura 2. Procesos de cognición social alterados y preservados en la esquizofrenia

Figura 3. Regiones cerebrales relacionadas con diferentes áreas de la cognición social

Capítulo 4. La cognición en la esquizofrenia (II)

Tabla 7. Módulos del Entrenamiento Metacognitivo (MCT)

Figura 4. Modelo de Vauth et al.

Figura 5. Modelo biopsicosocial de Brekke et al.

Figura 6. Modelo de Green y Nuechterlein 


\section{MARCO EXPERIMENTAL}

\section{Capítulo 5:}

Estudio 1. Los estilos atribucionales y el funcionamiento psicosocial en la esquizofrenia

Tabla 1. Características de la muestra

Tabla 2. Comparación entre grupos en ASQ

Tabla 3a. Correlaciones clínica y atribución

Tabla 3b. Correlaciones clínica, social y atribución

Tabla 4. Matriz de correlaciones

Tabla 5. Resumen de los modelos de regresión

\section{Capítulo 6:}

Estudio 2. Las dimensiones del apego como predictores del funcionamiento social en la esquizofrenia

Tabla 1. Características de la muestra

Tabla 2a. Comparación entre grupos en estilos de apego

Tabla 2b. Comparación entre grupos en CAMIR-R

Tabla 3. Correlaciones CAMIR-R y SFS-R

Tabla 4. Matriz de correlaciones

Tabla 5. Resumen de los modelos de regresión

Tabla 6. Análisis de mediación del funcionamiento social

Figura 1. Estilos de apego: estrategia primaria y secundaria

Figura 2. Modelo de mediación

Figura 3. Modelo de mediación áreas funcionamiento social 


\section{Capítulo 7:}

Estudio 3. La centralidad del apego seguro en una red interactiva entre los síntomas psicóticos, la cognición y las dimensiones del apego

Tabla 1. Características de la muestra

Tabla 2. Matriz de correlaciones modelo de redes

Figura 1. Modelo de redes

Figura 2. Gráfico centralidad del modelo

Figura 3. Gráfico estabilidad del modelo

Figura 4. Gráfico centralidad-estabilidad

\section{Capítulo 8:}

Estudio 4. Predictores del funcionamiento social en personas con esquizofrenia

Tabla 1. Características de la muestra

Tabla 2. Comparación entre grupos. Resumen del ANOVA

Tabla 3. Modelo de regresión lineal

Figura 1. Comparación entre grupos en cognición

Figura 2. Comparación entre grupos en apego

Figura 3. Comparación entre grupos en funcionamiento social 
MARCO TEÓRICO 


\section{Introducción}

La investigación que aquí se presenta, se enmarca dentro de los estudios sobre los trastornos del espectro de la esquizofrenia. Con ella se pretende aportar evidencia, tanto conceptual como empírica, sobre diversos constructos que han demostrado tener un valor fundamental en la vulnerabilidad, desarrollo y mantenimiento de los síntomas psicóticos, así como su valor predictivo sobre el funcionamiento social de las personas con trastornos del espectro de la esquizofrenia.

El objetivo fundamental de este trabajo es el de realizar una aproximación a la concepción actual de la esquizofrenia, atendiendo a su heterogeneidad, explorando la interacción de variables como la cognición, la cognición social y las relaciones de apego que influyen en la sintomatología y el funcionamiento social de las personas que padecen estos trastornos.

Nuestra investigación ha tratado de actualizar a modo de revisión, las principales bases teóricas que subyacen a estos fenómenos y que han sido abordadas desde enfoques de investigación experimental. En primer lugar, el marco teórico proporciona una conceptualización actual del espectro de la esquizofrenia, atendiendo a su clasificación diagnóstica, evaluación y programas de intervención. En segundo lugar, se revisa el apego desde una perspectiva que enlaza los patrones de apego adulto con la psicosis y el funcionamiento social. Finalmente, los dos últimos capítulos exploran la relación de los diferentes dominios de la cognición con el espectro de la esquizofrenia, aproximándose tanto a los modelos teóricos, las bases neurales y los sesgos cognitivos que parecen caracterizar su expresión.

Por otro lado, en el desarrollo del marco experimental, se presentan cuatro estudios que nos permitirán explorar más concretamente estos procesos. El primero de los estudios explora el papel de los estilos atribucionales en la sintomatología psicótica y en la predicción de los estados depresivos, así como en 
el funcionamiento social, poniendo a prueba la adecuación del Modelo de la Indefensión Aprendida. El segundo estudio explora los patrones de apego en la esquizofrenia como predictores del funcionamiento social, testando la aplicabilidad de una medida dimensional, el CAMIR-R, para evaluar el apego en una muestra de población psicótica. El tercer estudio explora las asociaciones entre diferentes dimensiones de apego, dominios de la cognición y síntomas psicóticos dentro de una red interactiva, utilizando una relativamente novedosa metodología: el análisis de redes. Por último, el cuarto estudio, se propone demostrar, por una parte, las diferencias en la gravedad de los déficit cognitivos a través de un continuo que va desde la normalidad hasta la esquizofrenia crónica y, por otro lado, la posible mediación de la severidad de los síntomas psicóticos en la influencia de los déficit cognitivos y los estilos de apego sobre el funcionamiento social. 


\section{Capítulo 1 Espectro de la esquizofrenia}

\subsection{Introducción}

La esquizofrenia es un trastorno mental grave que afecta a más de 21 millones de personas en todo el mundo, según las cifras de la Organización Mundial de la Salud (OMS), y se asocia a un conjunto de problemas de salud y psicosociales importantes (American Psychiatric Association, 2013). Se estima que la prevalencia de vida es de aproximadamente 0,7 \% (McGrath, Saha, Chant y Welham, 2008; Moreno-Küstner, Martín y Pastor, 2018; van der Werf et al., 2014), aunque las tasas varían según la localización geográfica y las características demográficas de la muestra. Por otro lado, la esquizofrenia se asocia con un aumento de la mortalidad, influida por otros trastornos psiquiátricos concurrentes, trastornos de abuso de sustancias, el suicidio, los problemas cardiovasculares y respiratorios, y otras afecciones médicas como la obesidad, la diabetes o el tabaquismo.

Este capítulo se centrará en presentar las diferentes características de los trastornos psicóticos, en concreto de la esquizofrenia, a partir de la descripción que ofrecen los dos sistemas de clasificación diagnóstica actuales, el DSM-5 y la CIE-11, así como los principales instrumentos de evaluación empleados para su detección y seguimiento, y los programas de intervención más habituales y con mayor apoyo empírico.

\subsection{Clasificaciones diagnósticas}

\subsubsection{Clasificación diagnóstica DSM-5}

El DSM-5 (APA, 2013) engloba la psicosis en su capítulo el espectro de la esquizofrenia y otros trastornos psicóticos (Tabla 1) y lo describe en relación a estos cinco dominios: 
1) Los delirios, creencias fijas que no son susceptibles de cambio a la luz de las pruebas en su contra. Los delirios más comunes en las psicosis son los delirios persecutorios, los delirios referenciales, los delirios de grandeza, los delirios erotomaníacos, los delirios nihilistas y los delirios somáticos. Son extravagantes cuando son claramente inverosímiles, incomprensibles y no proceden de experiencias de la vida corriente (p. ej., delirios que expresan una pérdida de control sobre la mente o el cuerpo).

2) Las alucinaciones, percepciones vívidas y claras que tienen lugar sin la presencia de un estímulo externo, y no están sujetas al control voluntario. Las alucinaciones auditivas (voces, conocidas o desconocidas) son las más frecuentes en el espectro de la esquizofrenia, aunque pueden darse en cualquier modalidad sensorial. Para diferenciar las alucinaciones de una experiencia normal, éstas deben ocurrir en un contexto con adecuado nivel de conciencia.

3) El pensamiento (discurso) desorganizado, cuyo síntoma debe ser lo suficientemente grave como para dificultar la comunicación de manera sustancial. Destacan el descarrilamiento, las asociaciones laxas, la tangencialidad, la incoherencia y la "ensalada de palabras".

4) El comportamiento motor muy desorganizado o anómalo (incluida la catatonía). Su manifestación es diversa, y se pueden observar problemas tanto para realizar cualquier tipo de comportamiento dirigido a un objetivo como para llevar a cabo actividades cotidianas. En concreto, en la catatonía (disminución marcada de la reactividad al entorno) destaca el negativismo, la postura rígida, inapropiada o extravagante, y el mutismo y estupor. Destacan también la excitación catatónica (actividad motora excesiva sin finalidad ni causa aparente), los movimientos estereotipados repetidos, la mirada fija, las muecas y la ecolalia.

5) Los síntomas negativos, especialmente prominentes en la esquizofrenia. Destacan principalmente: la expresión emotiva disminuida, disminución de la expresión de la emociones mediante el rostro, el contacto ocular, la 
entonación del habla (prosodia) y los movimientos que acompañan al discurso; y la abulia, disminución de las actividades, realizadas por iniciativa propia y motivadas por un propósito. Otros síntomas negativos son la alogia (disminución del habla), la anhedonia (disminución de la capacidad para experimentar placer a partir de estímulos positivos o de la degradación del recuerdo del placer experimentado previamente) y la asocialidad (aparente falta de interés por las interacciones sociales).

Más específicamente, la esquizofrenia se considera un síndrome clínico heterogéneo, siendo sustancial la variabilidad de características que se pueden presentar de un sujeto a otro. De este modo, los criterios diagnósticos comprenden un amplio conjunto de síntomas característicos que incluyen disfunciones cognitivas, conductuales y emocionales, aunque ningún síntoma concreto es patognomónico del trastorno. Asimismo, el diagnóstico conlleva la identificación de diversos signos y síntomas asociados con un deterioro del funcionamiento laboral o social. (Tabla 2)

Tabla 1. Clasificación DSM-5 para los trastornos psicóticos

\begin{tabular}{|c|c|}
\hline & Espectro de la esquizofrenia y otros trastornos psicóticos \\
\hline $301.22(\mathrm{~F} 21)$ & Trastorno esquizotípico (de la personalidad) \\
\hline $297.1(\mathrm{~F} 22)$ & $\begin{array}{l}\text { Trastorno delirantea,c } \\
\text { Especificar si: Tipo erotomaníaco, Tipo de grandeza, Tipo celotípico, Tipo } \\
\text { persecutorio, Tipo somático, Tipo mixto, Tipo no especificado } \\
\text { Especificar si: Con contenido extravagante }\end{array}$ \\
\hline $298.8(\mathrm{~F} 23)$ & $\begin{array}{l}\text { Trastorno psicótico breveb,c } \\
\text { Especificar si: Con factor(es) de estrés notable(s), Sin factor(es) de estrés } \\
\text { notable(s), Con inicio periparto }\end{array}$ \\
\hline 295.40 (F20.81) & $\begin{array}{l}\text { Trastorno esquizofreniformeb,c } \\
\text { Especificar si: Con características de buen pronóstico, Sin características de buen } \\
\text { pronóstico }\end{array}$ \\
\hline 295.90 (F20.9) & $\begin{array}{l}\text { Esquizofrenia }{ }^{\mathrm{a}, \mathrm{b}, \mathrm{c}} \\
\text { Trastorno esquizoafectivo }{ }^{\mathrm{a}, \mathrm{b}, \mathrm{c}} \\
\text { Especificar si: }\end{array}$ \\
\hline $\begin{array}{l}295.70(F 25.0) \\
295.70(F 25.1)\end{array}$ & $\begin{array}{l}\text { Tipo bipolar } \\
\text { Tipo depresivo }\end{array}$ \\
\hline
\end{tabular}


$293.81(\mathrm{~F} 06.2)$

293.89 (F06.0)

293.89 (F06.1)

293.89 (F06.1)

293.89 (F06.1)

298.8 (F28)

298.9 (F29)
Trastorno psicótico inducido por sustancias / medicamentos ${ }^{\mathrm{c}}$

Especificar si: Con inicio durante la intoxicación, Con inicio durante la abstinencia

Trastorno psicótico debido a otra afección médicac

Especificar si:

Con delirios

Con alucinaciones

Catatonía asociada a otro trastorno mental (especificador de catatonía)

Trastorno catatónico debido a otra afección médica

Catatonía no especificada

Otro trastorno del espectro de la esquizofrenia especificado y otro trastorno psicótico

Trastorno del espectro de la esquizofrenia no especificado y otro trastorno psicótico

aEspecificar si: Los siguientes especificadores del curso sólo se utilizarán después de un año de duración del trastorno: Primer episodio, actualmente en episodio agudo; Primer episodio, actualmente en remisión parcial; Primer episodio, actualmente en remisión total; Episodios múltiples, actualmente en episodio agudo; Episodios múltiples, actualmente en remisión parcial; Episodios múltiples, actualmente en remisión total; Continuo; No especificado.

bEspecificar si: Con catatonía (utilizar código adicional 293.89 [F06.1])

'Especificar la gravedad actual de los delirios, alucinaciones, habla desorganizada, conducta psicomotora anómala, síntomas negativos, deterioro cognitivo, depresión y síntomas maníacos.

Tabla 2. DSM-5: Criterios diagnósticos para la esquizofrenia

Esquizofrenia

Criterios diagnósticos

295.90 (F20.9)

A. Dos (o más) de los síntomas siguientes, cada uno de ellos presente durante una parte significativa de tiempo durante un período de un mes (o menos si se trató con éxito). Al menos uno de ellos ha de ser (1), (2) o (3):

1. Delirios

2. Alucinaciones.

3. Discurso desorganizado (p. ej., disgregación o incoherencia frecuente).

4. Comportamiento muy desorganizado o catatónico.

5. Síntomas negativos (es decir, expresión emotiva disminuida o abulia)

B. Durante una parte significativa del tiempo desde el inicio del trastorno, el nivel de funcionamiento en uno o más ámbitos principales, como el trabajo, las relaciones interpersonales o el cuidado personal, está muy por debajo del nivel alcanzado antes del inicio (o cuando comienza en la infancia o la adolescencia, fracasa la consecución del nivel esperado de funcionamiento interpersonal, académico o laboral).

C. Los signos continuos del trastorno persisten durante un mínimo de seis menses. Este período de seis meses ha de incluir al menos un mes de síntomas (o menos si se trató con éxito) que cumplan el Criterio A (es decir, síntomas de fase activa) y puede incluir períodos de síntomas prodrómicos o residuales. Durante estos períodos prodrómicos o residuales, los signos del trastorno se pueden manifestar únicamente por síntomas negativos o por dos o más síntomas enumerados en el Criterios A presentes de forma atenuada (p. ej., creencias extrañas, experiencias perceptivas habituales). 
D. Se han descartado el trastorno esquizoafectivo y el trastorno depresivo o bipolar con características psicóticas porque 1) no se han producido episodios maníacos o depresivos mayores de forma concurrente con los síntomas de fase activa, o 2) si se han producido episodios del estado de ánimo durante los síntomas de fase activa, han estado presentes sólo durante una mínima parte de la duración total de los períodos activo y residual de la enfermedad.

E. El trastorno no se puede atribuir a los efectos fisiológicos de una sustancia (p. ej., una droga o medicamento) o a otra afección médica.

F. Si existen antecedentes de un trastorno del espectro autista o de un trastorno de la comunicación de inicio en la infancia, el diagnóstico adicional de esquizofrenia sólo se hace si los delirios o alucinaciones notables, además de los otros síntomas requeridos para la esquizofrenia, también están presentes durante un mínimo de un mes (o menos si se trató con éxito).

Especificar si:

Los siguientes especificadores del curso de la enfermedad sólo se utilizarán después de un año de duración del trastorno y si no están en contradicción con los criterios de evolución diagnósticos.

- Primer episodio, actualmente en episodio agudo: La primera manifestación del trastorno cumple los criterios definidos de síntoma diagnóstico y tiempo. Un episodio agudo es el período en que se cumplen los criterios sintomáticos.

- Primer episodio, actualmente en remisión parcial: Remisión parcial es el período durante el cual se mantiene una mejoría después de un episodio anterior y en el que los criterios que definen el trastorno sólo se cumplen parcialmente.

- Primer episodio, actualmente en remisión total: Remisión total en el período después de un episodio anterior durante el cual los síntomas específicos del trastorno no están presentes.

- Episodios múltiples, actualmente en episodio agudo: Los episodios múltiples se pueden determinar después de un mínimo de dos episodios (es decir, después de un primer episodio, una remisión y un mínimo de una recidiva).

- Episodios múltiples, actualmente en remisión parcial

- Episodios múltiples, actualmente en remisión total

- Continuo: Los síntomas que cumplen los criterios de diagnóstico del trastorno están presentes durante la mayor parte del curso de la enfermedad, y los períodos sintomáticos por debajo del umbral son muy breves en comparación con el curso global.

- No especificado

Especificar si:

- Con catatonía

Especificar la gravedad actual:

La gravedad se clasifica mediante evaluación cuantitativa de los síntomas primarios de psicosis: ideas delirantes, alucinaciones, habla desorganizada, comportamiento psicomotor anormal y síntomas negativos. Cada uno de estos síntomas se puede clasificar por su gravedad actual (máxima gravedad en los últimos siete días) sobre una escala de 5 puntos de 0 (ausente) a 4 (presente y grave).

Nota: El diagnóstico de esquizofrenia se puede hacer sin utilizar este especificador de gravedad. 
A parte de estos criterios, existen otras características asociadas que apoyan el diagnóstico de esquizofrenia. Los individuos con esquizofrenia pueden mostrar problemas en el estado de ánimo, alteraciones del sueño, preocupaciones somáticas, problemas en la conducta alimentaria, ansiedad, fobia, así como déficits cognitivos y de la cognición social. Además, algunos individuos con psicosis muestran ausencia de introspección; es decir, la falta de conciencia de los síntomas de la esquizofrenia. Este síntoma puede estar presente a lo largo de todo el curso de la enfermedad. Aunque poco comunes, también pueden asociarse la hostilidad y la agresión. Por otro lado, cabe destacar que existe también una disfunción social, académica y laboral significativa, aunque el sujeto posea las habilidades cognitivas pertinentes.

Los índices de prevalencia de la esquizofrenia a lo largo de la vida son aproximadamente del 0,3 al 0,7\%. Los varones muestran más incidencia de síntomas negativos, que se asocian a un peor pronóstico, mientras que los síntomas del estado de ánimo y cuadros breves asociados a un mejor pronóstico, muestran un riesgo equivalente para ambos sexos.

Los síntomas psicóticos de la esquizofrenia suelen aparecer entre la adolescencia tardía y la mitad de la treintena, siendo el pico de inicio en la primera mitad de la veintena en los varones y a finales de la veintena en las mujeres; un inicio temprano suele predecir un peor pronóstico. Los casos de inicio tardío (a partir de los 40 años de edad) suelen ser más comunes en la mujeres. El inicio puede ser brusco o insidioso, pero la mayoría de los individuos presenta un desarrollo lento y gradual, refiriendo la mitad de ellos síntomas depresivos. En general, los síntomas psicóticos tienden a disminuir a lo largo de la vida, aunque los síntomas negativos tienden a ser más persistentes. Además, los déficits cognitivos que se asocian a la enfermedad pueden no mejorar a lo largo del curso de la misma.

Se consideran algunos factores de riesgo ambientales como son la estación de nacimiento, el medio urbano y los grupos étnicos minoritarios, así como una predisposición de alelos de riesgo como factor genético. Por otro lado, también 
deben considerarse las complicaciones del embarazo, los factores culturales y socioeconómicos, y otros aspectos relacionados con el género.

Las personas con esquizofrenia tienen una tasa alrededor del 5-6\% de fallecimiento por suicidio y aproximadamente del $20 \%$ de intento de suicidio al menos en una ocasión; no obstante, la mayoría de personas presenta una significativa ideación suicida. El riesgo de suicidio se mantiene alto a lo largo de toda la vida en los varones y en las mujeres, aunque podría ser especialmente elevado en los varones jóvenes con consumo comórbido de sustancias.

Es necesario establecer diagnóstico diferencial con los siguientes trastornos: trastorno depresivo mayor o trastorno bipolar con características psicóticas o catatónicas, trastorno esquizoafectivo, trastorno esquizofreniforme y trastorno psicótico breve, trastorno delirante, trastorno de la personalidad esquizotípica, trastorno obsesivo-compulsivo y trastorno dismórfico corporal, trastorno de estrés postraumático, trastorno del espectro autista o trastornos de la comunicación y otros trastornos mentales asociados con un episodio psicótico.

En cuanto a la comorbilidad, es significativa con los trastornos relacionados con sustancias, especialmente con el trastorno por consumo de tabaco (50\%). También existe comorbilidad con los trastornos de ansiedad, siendo las tasas elevadas en comparación con la población general de trastorno obsesivo-compulsivo y de trastorno de pánico. El trastorno de la personalidad esquizotípica o paranoide a veces puede preceder al inicio de una esquizofrenia. La esquizofrenia se asocia también a diversas afecciones médicas como el aumento de peso, la diabetes, el síndrome metabólico, enfermedades cardiovasculares y enfermedades pulmonares, por lo que la esperanza de vida en las personas con esquizofrenia es más corta.

\subsubsection{Clasificación diagnóstica CIE-11}

La CIE-11 (OMS, 2018) incluye dentro del capítulo sobre trastornos mentales y conductuales, la clasificación de síntomas para la esquizofrenia u otros trastornos psicóticos primarios (Tabla 3). En esta sección se indica que 
estos trastornos se caracterizan por distorsiones significativas en el juicio de la realidad y por alteraciones en el comportamiento que se manifiestan a través de síntomas positivos como delirios y alucinaciones persistentes, pensamiento desorganizado (que suele manifestarse como lenguaje desorganizado), comportamiento gravemente desorganizado, experiencias de pasividad y de control, síntomas negativos como afecto embotado o sin variación y abulia, y alteraciones psicomotoras. Los síntomas ocurren con suficiente frecuencia e intensidad para desviarse de lo esperado según las normas culturales y subculturales. Estos síntomas no se presentan como una característica de otro trastorno mental y del comportamiento (p. ej., trastorno del estado de ánimo, delirium o trastorno por consumo de sustancias). Las categorías en esta agrupación no deben utilizarse para clasificar la expresión de ideas, creencias o comportamientos que están sancionados culturalmente.

\section{Tabla 3. Clasificación CIE-11 para los trastornos psicóticos}

Esquizofrenia u otros trastornos psicóticos primarios

6A20 Esquizofrenia

Codificar: primer episodio, episodios múltiples, continua, otra esquizofrenia especificada, sin especificación

Codificar: actualmente sintomática, en remisión parcial, en remisión completa, sin especificación

6A21 Trastorno esquizoafectivo

Codificar: primer episodio, episodios múltiples, continuo, otro trastorno esquizoafectivo especificado, sin especificación

Codificar: actualmente sintomático, en remisión parcial, en remisión completa, sin especificación

6A22 Trastorno esquizotípico

6A23 Trastorno psicótico agudo y transitorio

Codificar: primer episodio, episodios múltiples, otro trastorno psicótico agudo y transitorio especificado, sin especificación

Codificar: actualmente sintomático, en remisión parcial, en remisión completa, sin especificación

6A24 Trastorno delirante

Codificar: actualmente sintomático, en remisión parcial, en remisión completa, sin especificación

6A25 Manifestaciones sintomáticas en los trastornos psicóticos primarios

Codificar: síntomas positivos, síntomas negativos, síntomas depresivos, síntomas maníacos, síntomas psicomotores, síntomas cognitivos 
Esquizofrenia u otros trastornos psicóticos primarios

6C40.6 Trastorno psicótico inducido por el consumo de alcohol

Codificar: con alucinaciones, con delirios, con síntomas psicóticos mixtos, sin especificación

6C41.6 Trastorno psicótico inducido por el consumo de cannabis

6C42.6 Trastorno psicótico inducido por el consumo de canabinoides sintéticos

6C43.6 Trastorno psicótico inducido por el consumo de opioides

6C44.6 Trastorno psicótico inducido por sedantes, hipnóticos o ansiolíticos

6C45.6 Trastorno psicótico inducido por cocaína

Codificar: con alucinaciones, con delirios, con síntomas psicóticos mixtos, sin especificación

6C46.6 Trastorno psicótico inducido por estimulantes, incluidas las anfetaminas, las metanfetaminas y la metcatinona

Codificar: con alucinaciones, con delirios, con síntomas psicóticos mixtos, sin especificación

6C47.6 Trastorno psicótico inducido por catinonas sintéticas

Codificar: con alucinaciones, con delirios, con síntomas psicóticos mixtos, sin especificación

6C49.5 Trastorno psicótico inducido por alucinógenos

6C4B.6 Trastorno psicótico inducido por inhalantes volátiles

6C4C.6 Trastorno psicótico inducido por MDMA u otras drogas relacionadas, incluida la MDA

6C4D.5 Trastorno psicótico inducido por drogas disociativas, incluidas la ketamina y la fenciclidina [PCP]

6C4E.6 Trastorno psicótico inducido por otras sustancias psicoactivas especificadas

6C4G.6 Trastorno psicótico inducido por sustancias psicoactivas desconocidas o no especificadas

6E61 Síndrome psicótico secundario

Codificar: con alucinaciones, con delirios, con alucinaciones y delirios, con síntomas con especificados

6A2Y Otra esquizofrenia u otros trastornos psicóticos primarios especificados

6A2Z Esquizofrenia u otros trastornos psicóticos primarios, sin especificación

En concreto, la esquizofrenia se caracteriza por trastornos en múltiples modalidades mentales, incluido el pensamiento (por ejemplo, ideas delirantes, desorganización en la forma de pensamiento), la percepción (por ejemplo, alucinaciones), la experiencia personal (por ejemplo, la experiencia de que los sentimientos, impulsos, pensamientos o comportamientos propios están bajo el control de una fuerza externa), la cognición (por ejemplo, problemas de atención, 
memoria verbal y cognición social), la volición o voluntad (por ejemplo, pérdida de motivación), el afecto (por ejemplo, expresión emocional embotada) y el comportamiento (por ejemplo, comportamientos que parecen bizarros o sin propósito, y respuestas emocionales impredecibles o inapropiadas que interfieren con la organización del comportamiento). Pueden presentarse alteraciones psicomotoras, incluida la catatonia. Las ideas delirantes persistentes, las alucinaciones persistentes, los trastornos del pensamiento y las experiencias de influencia, pasividad o control se consideran síntomas cerebrales. Los síntomas deben haber persistido durante al menos un mes para que se pueda asignar un diagnóstico de esquizofrenia. Los síntomas no son una manifestación de otra afección de salud (por ejemplo, un tumor cerebral) y no se deben al efecto de una sustancia o medicamento en el sistema nervioso central (por ejemplo, corticosteroides), incluida la abstinencia (por ejemplo, la abstinencia de alcohol). Por lo tanto, se excluyen el trastorno esquizotípico, el trastorno psicótico agudo y transitorio, y la reacción esquizofrénica. (Tabla 4)

Tabla 4. Codificación CIE-11 para la esquizofrenia

Criterios de codificación para la esquizofrenia

6A20.0 Esquizofrenia, primer episodio

El primer episodio de esquizofrenia se debe utilizar para identificar a las personas que experimentan síntomas que cumplen los requisitos de diagnóstico de la esquizofrenia (incluida la duración), pero que nunca antes han experimentado un episodio en el que se cumplan los requisitos de diagnóstico de la esquizofrenia.

Codificar si:

- actualmente sintomática (6A20.00)

- en remisión parcial (6A20.01)

- en remisión completa (6A20.02)

- sin especificación (6A20.0Z)

6A20.1 Esquizofrenia, episodios múltiples Codificar si:

- actualmente sintomática (6A20.10)

- en remisión parcial (6A20.11)

- en remisión completa (6A20.12)

- sin especificación (6A20.1Z) 


\begin{tabular}{ll}
\hline $\mathbf{6 A 2 0 . 2}$ & $\begin{array}{l}\text { Esquizofrenia continua } \\
\text { Codificar si: } \\
\\
\end{array}$ \\
& - actualmente sintomática (6A20.20) \\
& en remisión parcial (6A20.21) \\
& - sin especificación (6A20.2Z) \\
& Otra esquizofrenia especificada \\
$\mathbf{6 A 2 0 . Y}$ & Esquizofrenia, sin especificación
\end{tabular}

\subsubsection{Comparación entre clasificaciones}

Ambas clasificaciones han realizado cambios similares tanto en los criterios diagnósticos de la esquizofrenia al retirar los síntomas de primer rango de Schneider, como en la descripción de la esquizofrenia, ya que han retirado los subtipos del trastorno y han incorporado un especificador de síntomas en la evaluación de las manifestaciones clínicas de la esquizofrenia (delirios, alucinaciones, comportamiento psicomotor anormal, lenguaje desorganizado y síntomas negativos) (Valle, 2020). Además, el manejo de la catatonía ha sido también similar; por un lado, la CIE-11 considera que ésta puede producirse por trastornos mentales como la esquizofrenia, sustancias psicoactivas (incluidos medicamentos) y por condiciones médicas (Reed et al., 2019) y, por otro lado, el DSM-5 considera que la catatonía puede deberse tanto por trastornos mentales como por condiciones médicas, y tener una forma no especificada (Tandon et al., 2013). La modificación del epecificador de curso de la esquizofrenia ha sido también similar, lo que permite comparar los distintos patrones de curso que puede seguir la esquizofrenia entre ambos sistemas clasificatorios (Valle, 2020). Por otro lado, cabe destacar la influencia del enfoque dimensional en ambas clasificaciones, más evidente en el DSM-5 al incorporar en el título del capítulo el espectro de la esquizofrenia, pero también observable en la CIE-11 que, aunque conceptualiza la esquizofrenia bajo un enfoque categórico (Valle, 2020), se asemeja en el sistema de evaluación del especificador de síntomas (graduación de la severidad) a la clasificación DSM-5. 
No obstante, se distinguen algunas diferencias entre ambos sistemas clasificatorios: 1) el DSM-5 requiere adicionalmente a la duración de los síntomas psicóticos durante al menos un mes, que estos síntomas junto con los síntomas prodrómicos o residuales estén presentes por 6 meses (APA, 2013; Biedermann y Fleischhacker, 2016) y 2) la CIE-11 no considera los déficits funcionales específicos del trastorno, mientras que el DSM-5 mantiene el deterioro en la funcionalidad como un criterio diagnóstico (Gaebel, 2012).

El DSM-5 establece algunos cambios significativos en la concepción de la esquizofrenia en comparación al DSM-IV-TR: 1) se eleva el umbral de síntomas a, al menos, dos de los síntomas especificados, debiendo ser uno de estos síntomas: delirios, alucinaciones o habla desorganizada; 2) los criterios de diagnóstico pasan de identificarse en subtipos a utilizarse como especificadores para ayudar a proporcionar más detalles en el diagnóstico; 3) se elimina el criterio de que un solo delirio extravagante o alucinación auditiva de primer rango para cumplir con el criterio A, de esta forma cualquier tipo de delirio o alucinación tiene un mismo valor en el diagnóstico (Tandon, 2012).

Por lo que se refiere a la clasificación CIE-11, destacan tres cambios en la caracterización de la esquizofrenia en comparación a la CIE-10: 1) se retiran los subtipos de la esquizofrenia que se sustituyen por, 2) la introducción de un especificador de síntomas que registra información sobre la presencia o ausencia de síntomas, su curso longitudinal, la respuesta al tratamiento y el pronóstico en el trastorno (Tandon y Bruijnzeel, 2014). Sus categorías son los síntomas positivos, negativos, depresivos, maníacos, psicomotores y déficits cognitivos (Gaebel, Zielasek, Cleveland, 2013; Bucci, 2017; Keeley et al., 2018). 3) Se modifica el especificador de curso por la introducción de las categorías: primer episodio, múltiples episodios, curso continuo y no especificado, permitiendo las tres primeras especificar si el paciente presenta un episodio agudo, una remisión parcial o total de síntomas o es no especificado. Por otro lado, un cambio significante a nivel conceptual ha sido el restar preponderancia a los síntomas de primer rango propuestos por Scheneider (Ihara et al., 2009; Peralta y Cuesta, 1999), lo que permite hacer una valoración igual de estos síntomas con las 
alucinaciones y delirios de cualquier tipo como criterios diagnósticos de la esquizofrenia (OMS, 2018).

\subsection{Evaluación}

La evaluación del estado clínico en la esquizofrenia puede realizarse mediante diferentes instrumentos de evaluación. La misma clasificación DSM-5 (APA, 2013) aporta un instrumento de valoración de la severidad de los síntomas, que evalúa ocho dominios siguiendo una escala de 0 (no presente) a 4 (presente y grave) en función de los síntomas detectados los anteriores siete días. Los instrumentos más comunes utilizados para medir la psicopatología en la investigación, incluyen la Escala de Síndrome Positivo y Negativo (PANSS; Kay, Fiszbein y Opler, 1987), la Escala para la Evaluación de Síntomas Negativos (SANS; Andreasen, 1984a) y la Escala para la Evaluación de Síntomas Positivos (SAPS; Andreasen, 1984b), y la Escala Breve de Evaluación Psiquiátrica (BPRS; Overall y Gorham, 1962).

La evaluación no sólo se limita a los síntomas psicóticos sino que debe identificar y rastrear otros síntomas de la psicosis, incluidas las relacionadas con trastornos concurrentes. En este sentido, se recomienda para la medición del funcionamiento social y la calidad de vida la Escala de Discapacidad de la Organización Mundial de la Salud (WHODAS 2.0) para evaluar las dificultades de funcionamiento debidas a problemas de salud y de salud mental (Üstün et al. 2010). Otras opciones para evaluar el funcionamiento incluyen la Escala de Evaluación del Funcionamiento Social y Ocupacional (SOFAS; APA, 2000) y la Escala de Desempeño Personal y Social (Morosini, Magliano, Brambilla, Ugolini y Pioli, 2000). Por otro lado, el sistema PROMIS (del inglés, Patient-Reported Outcomes Measurement Information System), iniciativa del NIH, proporciona diferentes escalas de valoración que incluyen otros informantes para evaluar el nivel de funcionamiento del paciente, ya que las personas con esquizofrenia a menudo tienen una visión diferente de su funcionamiento que los miembros de la familia u otras personas involucradas en sus vidas (Harvey, 2011). 


\subsection{Plan individualizado y adherencia al tratamiento}

Los objetivos generales de la planificación del tratamiento se pueden resumir en: 1) promover y mantener la recuperación, 2) incrementar la calidad de vida y el funcionamiento adaptativo, y 3) reducir o eliminar los síntomas. Para lograr estos objetivos, es crucial identificar las aspiraciones del paciente, los objetivos del tratamiento y las preferencias relacionadas con el mismo, que en gran medida sirven para animar a los pacientes a observar y expresar sus preferencias sobre las opciones de tratamiento (Easter et al. 2017; Kemp, Zelle y Bonnie, 2015; Shields, Pathare, van der Ham y Bunders, 2014; Wilder, Elbogen, Moser, Swanson y Swartz, 2010). Las conversaciones con la persona afectada, el contacto con otros profesionales de la salud, miembros de la familia y otras personas relacionadas, es de gran utilidad para desarrollar una imagen completa del paciente y formular un plan de tratamiento individualizado y focalizado en la persona, a través de la toma de decisiones compartida. El plan de tratamiento debe estar actualizado, por lo que cobra todavía más importancia el compromiso de los familiares y de otras personas de apoyo (Cohen et al., 2013; Drapalski et al., 2018; Mueser et al., 2015). A nivel general, el plan de tratamiento se centrará en desarrollar tratamientos dirigidos a mejorar el funcionamiento, reducir los síntomas positivos y negativos, e intervenir sobre otros síntomas o trastornos concurrentes.

Por otro lado, también son importantes las estrategias para promover la adherencia al tratamiento en el desarrollo del plan individualizado (Ferrando, Owen y Levenson, 2014). La mejora de la adherencia puede conseguirse mediante diferentes enfoques. Algunas pautas destacables pueden ser llevar a cabo un seguimiento continuo de la adherencia a medida que avanza el tratamiento, centrarse en optimizar la eficacia del tratamiento, abordar los efectos secundarios y las preocupaciones sobre el tratamiento, ajustar la dosis para minimizar los efectos secundarios manteniendo la eficacia, proporcionando información sobre la enfermedad y sus tratamientos, participando en la toma de decisiones compartida, fomentando una sólida alianza terapéutica e involucrando 
a los miembros de la familia y otros apoyos comunitarios y sociales (Acosta et al., 2014; Hamann y Heres, 2019; Kane, Kishimoto y Correll, 2013; Rezansoff et al., 2017).

El plan de tratamiento debe también incluir una revaluación cuando existe riesgo de comportamientos agresivos o de acometer suicidio. A su vez, el plan individualizado debe abordar los trastornos de abuso de sustancias cuando estén presentes, como modelos de tratamiento integral (si es posible) o con la colaboración entre profesionales. Para pacientes que no reconocen la necesidad de tratamiento de un trastorno por uso de sustancias, se puede seguir un enfoque motivacional por etapas (Catley et al., 2016; Levounis, Arnaoutt y Marienfeld, 2017).

Por último, considerando que los síntomas depresivos son comunes en personas con esquizofrenia, deberán también abordarse con planificación y con un acurado diagnóstico diferencial. Parece ser que la adición a los fármacos antidepresivos resulta en pequeños beneficios sobre los síntomas de la depresión, la calidad de vida, así como en los síntomas positivos, negativos y generales (Helfer et al., 2016). Por otro lado, se debe considerar también en la planificación del tratamiento, el impacto de experiencias postraumáticas como la victimización violenta (de Vries et al., 2019; Morgan et al., 2016; Roy et al., 2014) o la adversidad infantil (Bonoldi et al., 2013; Schalinski et al., 2019; Trotta, Murray y Fisher, 2015; Varese et al., 2012).

\subsection{Intervención}

\subsubsection{Intervención farmacológica}

Los fármacos antipsicóticos son la intervención más utilizada en el tratamiento de la esquizofrenia, y su elección depende de muchos factores que son específicos del plan individual del paciente. En líneas generales, no existe una preferencia establecida por el uso de antipsicóticos de primera generación (FGA; del inglés, first generation antipsychotics) o de antipsicóticos de segunda 
generación (SGA; del inglés, second generation antipsychotics), ya que existe evidencia definitiva de que un antipsicótico en concreto tenga una eficacia consistentemente superior en comparación con otro, con la posible excepción de la clozapina. Por lo tanto, la elección de un antipsicótico particular dependerá de los probables beneficios y posibles efectos secundarios de las opciones de medicación. La Agencia Española de Medicamentos y Productos Sanitarios (AEMPS) proporciona una guía como marco de referencia para el uso de los antipsicóticos.

La clozapina se recomienda para personas con esquizofrenia resistente al tratamiento, que se basa en la persistencia de síntomas significativos a pesar de un tratamiento farmacológico adecuado (Howes et al., 2017). Por otro lado, el tratamiento con clozapina puede ser también eficaz para reducir el riesgo considerable de comportamiento suicida, independientemente de los criterios formales de resistencia al tratamiento, así como para reducir el comportamiento agresivo incipiente.

Con el uso de los antipsicóticos inyectables de larga duración (LAI; del inglés, long-acting injectable) existe una mayor seguridad de que un paciente reciba medicación continua, lo que permite prevenir la reaparición de los síntomas cuando se ha omitido alguna de las dosis (Correll et al., 2016; Velligan et al., 2010; West et al., 2008). Los LAI tienen una mejor adherencia al tratamiento y sus ventajas incluyen un menor riesgo de mortalidad, un reducido riesgo de hospitalización, y una tasa menor de interrupción del tratamiento.

Por último, otras intervención farmacológicas que cabe mencionar son los fármacos anticolinérgicos para la distonía aguda, la medicación para el tratamiento del parkinsonismo inducido por fármacos, fármacos para la acatisia aguda inducida por medicamentos, y los inhibidores VMAT2 para la discinesia tardía. 


\subsubsection{Intervenciones psicosociales}

La terapia cognitivo-conductual (TCC) para personas con esquizofrenia es la intervención psicosocial más utilizada y que presenta potenciales beneficios, como la mejora en la calidad de vida y el funcionamiento psicosocial, así como en la reducción de los síntomas centrales de la enfermedad y los síntomas positivos. Más específicamente, estos beneficios se relacionan a la TCC adaptada a la psicosis, que en concreto está enfocada a guiar a los pacientes para que desarrollen sus propias explicaciones a los supuestos cognitivos desadaptativos. Es decir, se centra en un enfoque colaborativo y sin prejuicios en la relación terapéutica, por lo que los pacientes pueden aprender a controlar las relaciones entre pensamientos, sentimientos, percepciones, comportamientos y síntomas y, de este modo, evaluar las percepciones, creencias y procesos de pensamiento que contribuyen a los síntomas (Beck y Rector, 2005; Beck, Rector, Stolar y Grant, 2009; Beck Institute, 2019; Hardy, 2019; Kingdon y Turkington, 2019; Landa, 2019; Lecomte, Leclerc y Wykes, 2016; Morrison, 2017; Turkington, Kingdon y Weiden, 2006; Wright, Turkington, Kingdon y Basco, 2009).

Los programas psicoeducativos varían en su formato, duración y ámbito, pudiendo impartirse en formato individual o grupal, y a menudo con miembros de la familia u otras personas relacionadas. El objetivo de estos programas es transmitir la información clave sobre el diagnóstico, los síntomas, las intervenciones psicosociales, el tratamiento farmacológico y los efectos secundarios, así como información sobre el estrés y el afrontamiento, planes de prevención de crisis, identificar señales de alerta, y prevención de recaídas y suicidio (Bäuml et al., 2006). Los programas de psicoeducación se asocian a mejoras en el funcionamiento global, disminución de recaídas, mejor adherencia al tratamiento y satisfacción con los servicios de salud mental.

Las intervenciones familiares, mediante un enfoque estructurado, se centran en la solución de problemas, manejo de la comunicación, apoyo emocional, estrategias de reducción de estrés y redes de apoyo social (McDonagh et al., 2017; McFarlane, 2016; Mueser, Deavers, Penn y Cassisi, 2013). Aunque 
pueden ser útiles durante cualquier fase del tratamiento, parecen ser particularmente relevantes al principio del curso de la esquizofrenia (McFarlane, 2016). Pueden reducir los síntomas centrales de la enfermedad, minimizar las recaídas y la rehospitalización, así como mostrar beneficios para los miembros de la familia al reducir la carga y la angustia y mejorar la relación intrafamiliar (McDonagh et al., 2017; McFarlane, 2016; Sin et al., 2017).

Los programas comunitarios de tratamiento asertivo se centran en un enfoque multidisciplinar basado en el equipo donde los pacientes reciben atención individualizada desde sus casas, lugares de trabajo u otras ubicaciones comunitarias. Esta intervención parece beneficiar a la persona a vivir independientemente y mantener un trabajo.

La terapia de remediación cognitiva se asocia con mejoras moderadas en aspectos específicos de la cognición (Harvey, McGurk, Mahncke y Wykes, 2018; Wykes, Huddy, Cellard, McGurk y Czobor, 2011), y parece tener efectos positivos en el funcionamiento psicosocial y los síntomas negativos (McDonagh et al., 2017). El entrenamiento en habilidades sociales parece mejorar el funcionamiento social, así como los síntomas centrales y los síntomas negativos, y reduce las recaídas y la rehospitalización (McDonagh et al., 2017). Mientras que la psicoterapia de apoyo tiene como objetivo ayudar a los pacientes a afrontar los síntomas, mejorar las habilidades adaptativas y aumentar la autoestima, y suele llevarse a cabo junto al tratamiento farmacológico.

Por último, otras intervenciones, como el empleo con apoyo, producen mejoras en los resultados laborales. $\mathrm{Y}$ los programas para desarrollar habilidades y las intervenciones centradas en la recuperación, proporcionan mayor activación y calidad de vida, y pueden promover una mayor recuperación, esperanza y empoderamiento (Le Boutillier et al., 2011; Mueser et al., 2013; Thomas, Despeaux, Drapalski y Bennet, 2018). 


\section{Capítulo 2 Apego y psicosis}

\subsection{Introducción}

Los patrones de apego y las adversidades en la infancia son relevantes para entender el papel de los factores ambientales y psicosociales en la vulnerabilidad y el desarrollo de las psicosis (Sheinbaum, Bedoya, Ros-Morente, Kwapil y Barrantes-Vidal, 2013; Bailey et al., 2018). En particular, la investigación se ha centrado en encontrar los mecanismos a través de los cuales, el trauma interpersonal infantil agrava el riesgo de desarrollar psicosis (Bailey et al., 2018).

Se considera que a través de las relaciones de apego se forman fuertes vínculos emocionales con los cuidadores en los primeros 1-2 años de vida (Bowlby, 1969). Estas relaciones pueden conducir a que en las etapas infantiles se experimente estrés y ansiedad por separación cuando los cuidadores están ausentes, siendo este malestar únicamente paliado por estas figuras de apego, no por cualquier otro cuidador (Harder, 2014). De acuerdo con la teoría del apego parece existir una necesidad evolutiva para el apego relacionada con el incremento de la supervivencia, ya que a través de los contactos cercanos se generan las condiciones óptimas para el desarrollo cognitivo y socioemocional del niño y la niña. Las experiencias en las relaciones de apego conllevan eventualmente al desarrollo de un modelo de trabajo interno para la regulación del afecto, la exploración, y el funcionamiento interpersonal; esto es, un patrón de apego que tiende a ser relativamente estable en el tiempo.

Este capítulo tiene como objetivo conceptualizar el constructo de apego desde la perspectiva de investigación actual, prestando atención a las características que lo relacionan con las psicosis, la cognición y el funcionamiento psicosocial. 


\subsection{Modelos teóricos del apego}

\subsubsection{Teoría del apego}

La teoría del apego define el apego como la tendencia de los seres humanos a crear fuertes lazos afectivos con determinadas personas en particular (Bowlby, 1977). Más en concreto, el apego se conceptualiza como la propensión de los seres humanos a formar vínculos afectivos fuertes con los demás y de extender las diversas maneras de expresar emociones de angustia, depresión y enfado cuando son abandonados o viven una separación o pérdida (Bowlby, 1988). Las conductas de apego son desencadenadas por amenazas del entorno, angustia, enfermedad o fatiga; y son definidas como cualquier forma de conducta que resulta en el individuo que recupera o mantiene el contacto con su figura de apego (Bowlby, 1982). La teoría del apego sugiere que la calidad de las primeras experiencias con los cuidadores influyen de tal manera que estas relaciones se internalizan como representaciones cognitivo-afectivas o modelos de trabajo internos de sí mismo y de los demás, que proporcionan los patrones para las relaciones sociales posteriores que permanecen durante el curso de la vida (Bowlby, 1980; Ainsworth, 1989; Bifulco y Thomas, 2012).

Algunos autores proponen que este modelo proporciona un fuerte marco teórico para entender el impacto de las primeras experiencias traumáticas a través de una distorsión de los esquemas mentales, desregulación afectiva y alteración de los patrones interpersonales (Hardy et al., 2016). Según la hipótesis de Rajkumar (2014) de apego y desarrollo cognitivo de la esquizofrenia, el apego también puede representar un constructo capaz de mediar entre los niveles de desarrollo, neurobiológicos e interpersonales en psicosis.

\subsubsection{Modelos de trabajo}

Tomando como base la teoría del apego, Bartholomew y Horowitz (1991) desarrollaron dos tipos de modelos de trabajo: 1) El modelo de sí mismo (del inglés: model of the self) se define como el grado en que un individuo ha 
internalizado un sentido de su propia autoestima y, en consecuencia, espera que los demás reaccionen ante él de una manera positiva. Este modelo se asocia con el grado de ansiedad y dependencia experimentado en relaciones cercanas; es decir, indica la capacidad del individuo de regular el grado de dependencia hacia los demás. 2) El modelo de los demás (del inglés: model of the others) se refiere al grado en que un individuo espera que otros puedan estar disponibles y mostrar apoyo. Este modelo se expresa en términos de confianza y se asocia a la tendencia a buscar o evitar cercanía en las relaciones (Grifin y Bartholomew, 1994).

El modelo de Bartholomew y Horowitz resulta en cuatro estilos de apego: 1) El apego seguro, que consiste en un modelo de sí mismo y de los demás positivo, que indica un juicio de valor junto a una expectativa de que los otros son generalmente tolerables y receptivos. 2) El apego preocupado, que relaciona de manera negativa los dos modelos; esto es, un juicio de ausencia de mérito con una evaluación positiva de los demás, lo que conlleva a la persona a tener que esforzarse para autoaceptarse mediante la aceptación de la valoración de los demás. 3) El apego evitativo se caracteriza por un modelo positivo de sí mismo y un modelo negativo de los otros; es decir, un juicio de amor-mérito relacionado con una negativa propensión hacia los otros. De esta manera, estas personas tratan de protegerse contra la decepción evitando las relaciones cercanas manteniendo su sentido de independencia e invulnerabilidad. 4) El apego temeroso, que consiste en un sentido negativo de ambos modelos; un juicio de descrédito unido a una expectativa de que los demás estarán negativamente dispuestos. Así, estas personas evitan participar estrechamente con los demás para protegerse contra un posible rechazo anticipado. (Figura 1)

Dado que el sistema de apego humano organiza experiencias personales dentro de modelos internos de trabajo que filtran y canalizan nuevas experiencias, un individuo tiende a seguir la misma trayectoria a lo largo de la vida (Mayseless, 1996). 


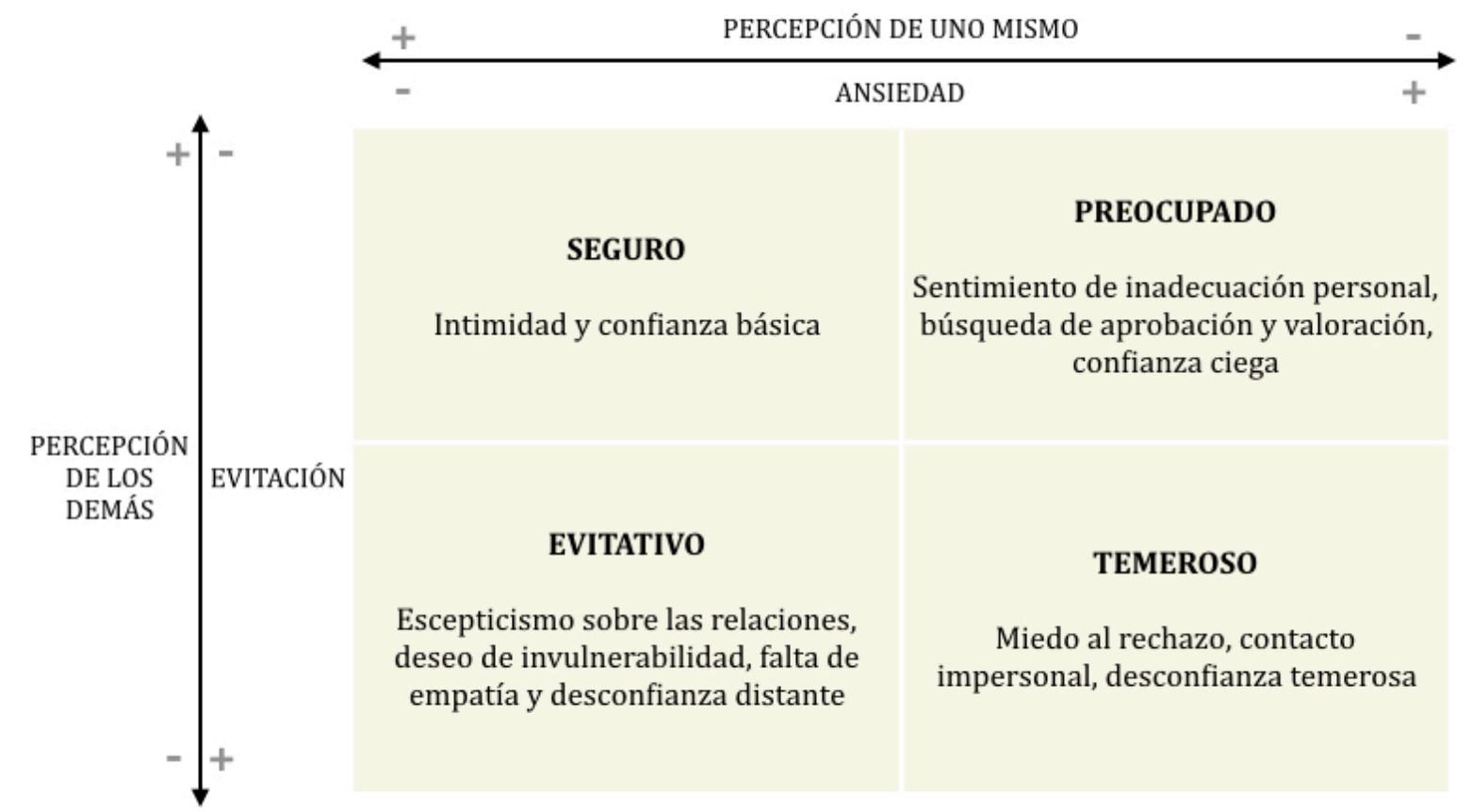

Figura 1. Modelo de Bartholomew y Horowitz (1991).

Otro de los modelos a considerar es el de Stein et al. (2002) que combina las dimensiones ansiedad y evitación con las categorías tradicionales de apego. Este modelo representa los estilos preocupado y evitativo como alternativas de estrategia para enfrentar las relaciones interpersonales en la ausencia de una habilidad para formar relaciones cercanas y seguras (Martínez y Santelices, 2005). El primer componente, inseguridad (seguridad vs temor), se relaciona con el nivel de ansiedad que experimenta la persona frente a las relaciones interpersonales. El segundo, estrategia (rechazante vs preocupado), se relaciona con las estrategias que los individuos utilizan para enfrentar las dificultades con las relaciones interpersonales. De ellos se derivan tres supuestos: 1) baja inseguridad que no necesita la estrategia de enfrentamiento, 2) moderada a gran inseguridad que requiere de estrategias específicas (preocupadas o evitativas) para sustentar las relaciones, y 3) extrema inseguridad (temor) que expresa un sistema desorganizado de apego que evita una estrategia específica. 


\subsection{Evaluación de los estilos de apego}

En general, la literatura describe tres patrones organizados del apego adulto (Hesse, 2008; Harder, 2014), entendidos como adaptaciones del tipo de cuidado recibido en la infancia; estos son: 1) Apego seguro, caracterizado por una regulación adaptativa del afecto, una capacidad de ser emocionalmente cercano, pero a la vez autónomo en las relaciones interpersonales y de comprender los estados mentales (mentalización). 2) Apego evitativo, se caracteriza por una tendencia a desactivar las emociones y la atención hacia los estados mentales del yo y del otro, por lo que se prefiere mantener a los demás a distancia, valorando los éxitos por encima de las relaciones. Parece ser que la persona se ha adaptado a una expresión de angustia debida a las experiencias tempranas de rechazo, por lo que expresa una regulación excesiva de las emociones y estrategias de distracción con el fin de hacer frente a la angustia solo. 3) Apego preocupado, describe una expresión exagerada de las emociones y mantiene la atención hacia las figuras de apego a costa de explorar y desarrollar su propia autonomía. Las emociones tienden a desregularse y los sentimientos positivos a menudo se mezclan con sentimientos de ira y ansiedad. 4) Además de estos tres patrones, algunos individuos desarrollan un carácter de apego desorganizado caracterizado por fracturas transitorias o por una profunda ruptura de un patrón de apego organizado. No hay una estrategia para regular con éxito las emociones, lo que deriva a incoherencias en los estados mentales. Este tipo de apego se ha vinculado a experiencias adversas en la infancia.

La teoría de apego ha generado diversos instrumentos para evaluar diferencias individuales en el apego adulto (Lacasa y Muela, 2014). Distintas medidas evalúan diferentes niveles de consciencia sobre las representaciones de apego, como es el caso de la entrevista Adult Attachment Interview (AAI) (George, Kaplan y Main., 1985; Crowell y Treboux, 1995; Crowell, 2014) para evaluar los patrones clásicos del apego, mencionados anteriormente. Este instrumento narrativo facilita la evaluación de los aspectos no conscientes del apego a través de claves externas de respuestas. Consiste en una entrevista semi-estructurada 
de mínimo una hora de duración que determina el apego a partir de las narraciones que la persona hace sobre sus relaciones con las figuras de apego (Lucena, Cifre, Castillo-Garayoa y Aragonés, 2015). Además, permite guiar la intervención terapéutica y evaluar su resultado (Steele, Steele y Murphy, 2009; Yárnoz, Alonso-Arbiol, Plazaola y Sainz de Murieta, 2001; Yárnoz y Comino, 2011). Recientemente, Lucena et al. (2015) crearon el Perfil Clínico de Apego (PCA-n), un sistema de categorías para evaluar el estilo de apego a partir de las narrativas que el paciente hace sobre sí mismo y sobre los demás en el curso de entrevistas clínicas o sesiones de psicoterapia.

Por otro lado, los cuestionarios de tipo autoinforme se centran en las ideas conscientes sobre el apego y las relaciones (Molina, Casonato, Sala y Testa, 2018). Para la evaluación del apego adulto, encontramos desde los clásicos, el cuestionario de relación-CR (Bartholomew y Horowitz, 1991) y el cuestionario del estilo de apego adulto (Hazan y Shaver, 1987), hasta los instrumentos más actuales como, el cuestionario del apego adulto (CCA; Melero y Cantero, 2008), la versión española de la Experiences in Close Relationships (ECR) de Brennan, Clark y Shaver (1998) (ECR-S; Alonso-Arbiol, Balluerka y Shaver, 2007) y la versión revisada (ECR-R; Fraley, Waller y Brennan, 2000; Fernández-Fuertes, Orgaz, Fuertes y Carcedo, 2011), la escala de preferencias y expectativas en las relaciones interpersonales cercanas (EPERIC; Fontanil, Ezama y Alonso, 2013), o la adaptación española del cuestionario para la evaluación del apego CAMIR (Pierrehumbert et al., 1996; Balluerka, Lacasa, Gorostiaga, Muela y Pierrehumbert, 2011). Aunque el uso de autoinformes es más sencillo y económico, pueden resultar simples en la evaluación de las representaciones inconscientes y en el caso concreto del apego temeroso (Molina et al., 2018). No obstante, la literatura está de acuerdo en que ambos tipos de instrumentos de evaluación, entrevista y autoinformes, son útiles para evaluar los diferentes tipos de constructo (Roisman et al., 2007).

Más en concreto, destacamos la versión reducida del cuestionario para la evaluación del apego CAMIR-R (Balluerka et al., 2011) por ser el instrumento elegido para evaluar el apego en los estudios empíricos llevados a cabo en esta 
investigación. El mérito de este instrumento radica en la riqueza y complejidad del modelo teórico que lo sustenta: un modelo del yo y del otro que impulsa las relaciones personales a lo largo de la vida y de la edad adulta, generadas por la experiencia temprana con las personas cuidadoras; aunque estas experiencias pueden modificarse por posteriores experiencias en las relaciones (Molina et al., 2018). Por otro lado, el CAMIR-R puede administrarse independientemente de la estructura familiar de la persona; proporciona medición continua de las dimensiones del apego, lo que permite evaluar los fenómenos clínicos más a fondo que con instrumentos basados en categorías; y presenta adecuadas propiedades psicométricas para evaluar el apego en contextos clínicos y de investigación con adolescentes y adultos, tanto en su versión original como reducida (Balluerka et al., 2011; Molina et al., 2018).

\subsection{Relaciones del apego con el espectro de las psicosis}

La mayoría de la investigación se ha centrado en pacientes crónicos y poco se sabe acerca del rol del apego en los estadios iniciales de la psicosis (Quijada, 2012). En general, la mayoría de las personas con trastornos del espectro de la esquizofrenia tienden a mostrar un estilo de apego inseguro (Bailey et al., 2018); no obstante, las asociaciones con los síntomas no están del todo claras. Algunos estudios relacionan estas asociaciones entre el apego evitativo y las dimensiones positiva y negativa de los síntomas psicóticos y el apego ansioso con los síntomas

positivos (Chatziioannidis et al., 2019), mientras el apego preocupado y el apego temeroso se relaciona con los síntomas positivos, aunque no se hallan relaciones con el apego evitativo (Ponizovsky, Vitenberg, Baumgarten-Katz y Grinshpoon, 2013). La investigación ha mostrado que el apego evitativo oscila entre el 48 \% y el $71 \%$ en personas con trastornos del espectro psicótico, el apego preocupado oscila entre el 12-20 \%, y el apego seguro entre el 27-32 \% en comparación con muestras de población general (Harder, 2014).

Más en concreto, el apego evitativo se asocia significativamente con los síntomas positivos y negativos, paranoia, experiencias delirantes (Varghese, Scott 
y McGrath, 2008; Korver-Nieberg, Berry, Meijer y MacBeth, 2014; Wickman, Sitko y Bentall, 2015) y angustia al escuchar voces (Berry et al., 2012; Pilton et al., 2016). Además, las formas de apego inseguro parecen correlacionar con el sesgo atribucional (Donohoe et al., 2008) y la mentalización (MaBeth, Gumley, Schwannauer y Fisher, 2011; Debbané et al., 2016; Gumley, Taylor, Schwannauer y MacBeth, 2014); se relacionan con un pobre ajuste premórbido, mayores problemas interpersonales y de funcionamiento psicosocial (Berry, Barrowclough y Wearden, 2008); se asocian con la cognición social que sirve como mediador entre el apego inseguro y los problemas clínicos y de funcionamiento en la psicosis (Korver-Nieberg et al., 2014); y también se relacionan con individuos sanos con experiencias psicóticas (Gawęda et al., 2018; Blair et al., 2018). Por otro lado, el apego seguro parece estar relacionado en la forma en que las personas con psicosis interactúan con los servicios de salud mental (McGonagle et al., 2019) y las necesidades de relación íntima de los usuarios con psicosis (White, Haddock y Varese, 2020).

Existe un claro predominio de patrones de apego inseguro, principalmente de apego temeroso y de rechazo-evitación, en pacientes psicóticos (Carr, Hardy y Fornell-Ambrojo, 2018). Por otro lado, los estudios destacan el impacto y el riesgo del trauma infantil en el desarrollo de experiencias psicóticas, principalmente alucinaciones y delirios (Bayley et al., 2018; Rösler et al., 2015), y en el desarrollo de habilidades cognitivas sociales, que pueden representar un mediador entre las adversidades tempranas de la vida y la severidad de los síntomas posteriores (Rokita, Dauvermann y Donohoe, 2018). Además, los factores psicológicos están relacionados con el apego inseguro y las vías neurobiológicas reconocidas que se vinculan con el riesgo de desarrollar psicosis y la vulnerabilidad a la esquizofrenia (Debbané et al., 2016; Pilton et al., 2016; Popovic et al., 2019; Aydin et al., 2019; Speck et al., 2019). 


\section{Capítulo 3 La cognición en la esquizofrenia (I) ${ }^{1}$ :}

Concepto, métodos de evaluación y correlatos neurales

\subsection{Introducción}

Las manifestaciones clínicas de la esquizofrenia involucran un rango de limitaciones interrelacionadas que afectan de forma "semindependiente" a los síntomas y deficiencias en diferentes dominios de la cognición incluyendo, además de la cognición social, a la neurocognición y a la metacognición, y está comúnmente aceptado que todo ello afecta de algún modo al funcionamiento psicosocial (Green, Horan y Lee, 2015).

Por otro lado, algunos estudios muestran que los síntomas y los déficits neurocognitivos se influyen mutuamente, y que se relacionan a su vez con la cognición social y la metacognición (Schmidt, Mueller y Roder, 2011; Lysaker y Dimaggio, 2014; Lysaker, Hamm, Hasson-Ohayon, Pattison y Leonhardt, 2018; Lysaker y Hasson-Ohayon, 2014). No obstante, no está claro si estos constructos se afectan entre sí, ni si algunos de estos fenómenos desempeñan un papel más o menos central que otros a medida que interactúan.

Las alteraciones en estos procesos se han vinculado a perturbaciones estructurales y funcionales en los constituyentes del llamado "cerebro social". Este incluye un conjunto de áreas prefrontales mediales, parietales y temporales que se han asociado a algunas anomalías en la teoría de la mente (ToM, del inglés theory of mind), la percepción de emociones y la capacidad para considerar la perspectiva de los otros, fenómenos comúnmente encontrados en la esquizofrenia (Green et al., 2015; García, Aliste y Soto, 2018).

\footnotetext{
${ }^{1}$ El presente capítulo ha sido publicado como artículo de revisión en:

Pena-Garijo, J., \& Monfort-Escrig, C. (2020). Cognition in schizophrenia. State of the art (Part I): assessment methods and neural correlates. Revista Asociación Española de Neuropsiquiatría, 40, 109-130. Disponible en: http://www.revistaaen.es/index.php/aen/article/view/17121
} 
En este y en el posterior capítulo se intentarán describir los diferentes aspectos de la cognición (social y no social), de las teóricas bases neurales de dichos procesos, de los sesgos cognitivos característicos de la esquizofrenia, de su evaluación y de sus implicaciones con el funcionamiento social, así como las posibles intervenciones terapéuticas para mejorarlos. En el presente capítulo, se revisarán los conceptos de neurocognición y cognición social, así como se describirán los métodos de evaluación recomendados y los posibles correlatos neurales implicados, atendiendo a la investigación más reciente.

\subsection{La neurocognición en la esquizofrenia}

El concepto de neurocognición hace referencia a los procesos de enlazar y evaluar información, e incluye habilidades como la velocidad de procesamiento, la atención, el aprendizaje, la memoria verbal y visual, la memoria de trabajo, y el razonamiento y la resolución de problemas (Nuechterlein et al., 2004; Roder y Medalia, 2010; Schmidt et al., 2011). Los déficits en la esquizofrenia se asocian con el bloqueo o deterioro de importantes funciones mentales que se caracterizan por: iniciar y mantener actividades; fluidez del habla y pensamiento; expresión de emociones; comunicación no verbal; sentimientos de alegría y placer; e interacción con otras personas. Parece ser que la presencia de estos déficits conduce a la apatía y reduce la expresión emocional (Wojciechowska, Kłosińska, Kur y Wichniak, 2016).

En las últimas décadas ha habido un interés significativo en la identificación de los factores que pueden moderar o influir en la clínica y el funcionamiento de las personas con esquizofrenia. La medida del desempeño en habilidades cognitivas como la memoria, la atención y las funciones ejecutivas, se ha relacionado de manera consistente con los resultados en recuperación funcional y con los síntomas clínicos (Lepage, Bodnar y Bowie, 2014). Sin embargo, los mismos autores que describen esta relación ponen de manifiesto que hacer comparaciones entre diferentes estudios es, cuando menos, 
cuestionable. Una posible limitación tiene que ver con las discrepancias en la propia definición de las variables clínicas y de funcionamiento. Por ejemplo, los dominios cognitivos no se miden utilizando las mismas pruebas neuropsicológicas. Y, además, la distinción entre la competencia para realizar las tareas cognitivas (condición "experimental") y lo que realmente se hace en la vida real, ha recibido poca atención, a pesar de su evidente importancia (Lepage et al., 2014).

\subsubsection{Evaluación}

Con el fin de paliar algunas de estas limitaciones, el NIMH desarrolló la Iniciativa de Medición y Tratamiento para Mejorar la Cognición en la Esquizofrenia: MATRICS (del inglés: Measurement and Treatment Research to Improve Cognition in Schizophrenia). Una de las claves de esta iniciativa fue la creación de la MATRICS Consensus Cognitive Battery (MCCB) para ensayos clínicos sobre esquizofrenia con el objetivo de evaluar la mejora cognitiva tras implementar un tratamiento (Kern et al., 2011; Horan, Blanchard, Clark y Green, 2008; Green et al., 2008). Esta batería estándar incluyó la cognición social como uno de los dominios, a pesar de que en el momento en que se tomó la decisión de incluirla, había relativamente pocos estudios sobre ella (Green et al., 2008). Cabe destacar, que la cognición social parece estar afectada en personas con esquizofrenia y es de importancia crítica para el desempeño social y vocacional de las personas que la padecen, teniendo correlaciones algo más altas que los déficits cognitivos no sociales (Fett et al., 2011). Por este motivo, la cognición social se ha convertido en un objetivo de tratamiento en la esquizofrenia, como lo demuestra el desarrollo creciente de intervenciones psicosociales (Kamel y AlQahtani, 2019; Kurtz, Gagen, Rocha, Machado y Penn, 2016; Liu, Tang, Hung, Tsai y Lin, 2018) y tratamientos farmacológicos prometedores (Vita et al., 2018).

El objetivo de la MCCB es proporcionar una evaluación relativamente breve de los dominios cognitivos relevantes para la esquizofrenia y trastornos relacionados, y ha sido diseñada para ser utilizada: a) como medida de resultado 
de ensayos clínicos con fármacos para la mejora de la cognición en la esquizofrenia, b) como medida de resultado para estudios de recuperación cognitiva, c) como medida de cambio cognitivo en la aplicación de pruebas repetidas y, d) como punto de referencia cognitiva para estudios de nointervención en esquizofrenia y trastornos relacionados (Rodríguez-Jiménez et al., 2012a).

En resumen, es una batería de instrumentos de evaluación de la cognición en la esquizofrenia y consta de pruebas que evalúan los siguientes 7 dominios: a) velocidad de procesamiento, b) atención y vigilancia, c) memoria de trabajo (verbal y no verbal), d) aprendizaje verbal, e) aprendizaje visual, f) razonamiento y resolución de problemas, y g) cognición social. Requiere aproximadamente 70 minutos para su administración y consta de 10 pruebas independientes (Tabla 5). La batería ha sido estandarizada para su uso en población española (RodríguezJiménez et al., 2012b).

Tabla 5. Instrumentos de evaluación (MATRICS)

\begin{tabular}{|c|c|c|}
\hline Dominio & Prueba & Descripción \\
\hline \multirow{3}{*}{$\begin{array}{l}\text { Velocidad de } \\
\text { procesamiento }\end{array}$} & $\begin{array}{l}\text { Breve evaluación de la } \\
\text { cognición en la } \\
\text { esquizofrenia (BACS): } \\
\text { codificación de símbolos }\end{array}$ & $\begin{array}{l}\text { Prueba cronometrada de papel y lápiz } \\
\text { en la que el sujeto utiliza una clave para } \\
\text { escribir dígitos que corresponden a } \\
\text { símbolos sin sentido. }\end{array}$ \\
\hline & $\begin{array}{l}\text { Fluidez verbal por } \\
\text { categorías: nombres de } \\
\text { animales }\end{array}$ & $\begin{array}{l}\text { Prueba oral en la que el sujeto nombra } \\
\text { tantos animales como puede durante } 1 \\
\text { minuto. }\end{array}$ \\
\hline & Trail Making Test: Parte A & $\begin{array}{l}\text { Prueba cronometrada de papel y lápiz } \\
\text { que consiste en dibujar una línea para } \\
\text { conectar círculos numerados } \\
\text { consecutivamente colocados } \\
\text { irregularmente en una hoja de papel. }\end{array}$ \\
\hline Atención / Vigilancia & $\begin{array}{l}\text { Test de rendimiento } \\
\text { continuo-Pares idénticos } \\
\text { (CPT-IP) }\end{array}$ & $\begin{array}{l}\text { Evaluación computarizada de atención } \\
\text { sostenida en la que el sujeto presiona un } \\
\text { botón de respuesta a números } \\
\text { coincidentes consecutivos. }\end{array}$ \\
\hline $\begin{array}{l}\text { Memoria de trabajo } \\
\text { (no verbal) }\end{array}$ & $\begin{array}{l}\text { Escala de memoria de } \\
\text { Wechsler-III (WMS-III): } \\
\text { span espacial }\end{array}$ & $\begin{array}{l}\text { Mediante un tablero y } 10 \text { cubos } \\
\text { distribuidos irregularmente, la persona } \\
\text { debe reproducir la misma secuencia (o } \\
\text { inversa) que la persona administradora } \\
\text { de la prueba. }\end{array}$ \\
\hline
\end{tabular}




\begin{tabular}{|c|c|c|}
\hline Dominio & Prueba & Descripción \\
\hline $\begin{array}{l}\text { Memoria de trabajo } \\
\text { (verbal) }\end{array}$ & $\begin{array}{l}\text { Escala de memoria de } \\
\text { Wechsler-III (WMS-III): } \\
\text { span de Letra-Número }\end{array}$ & $\begin{array}{l}\text { Prueba oral que consiste en reordenar } \\
\text { mentalmente cadenas de números y } \\
\text { letras, y repetírselas a la persona que } \\
\text { administra la prueba. }\end{array}$ \\
\hline Aprendizaje verbal & $\begin{array}{l}\text { Test de aprendizaje verbal } \\
\text { de Hopkins-Revisado } \\
\text { (HVLT-R) }\end{array}$ & $\begin{array}{l}\text { Test oral donde se presenta una lista de } \\
12 \text { palabras de tres categorías } \\
\text { taxonómicas y se pide al sujeto que } \\
\text { recuerde la mayor cantidad posible de } \\
\text { palabras después de cada una de las tres } \\
\text { pruebas de lenguaje. }\end{array}$ \\
\hline Aprendizaje visual & $\begin{array}{l}\text { Prueba de memoria } \\
\text { visuoespacial breve- } \\
\text { Revisada (BVMT-R) }\end{array}$ & $\begin{array}{l}\text { El objetivo de esta prueba es reproducir } \\
\text { seis figuras geométricas de memoria. }\end{array}$ \\
\hline $\begin{array}{l}\text { Razonamiento y } \\
\text { solución de } \\
\text { problemas }\end{array}$ & $\begin{array}{l}\text { Batería de evaluación } \\
\text { neuropsicológica: } \\
\text { laberintos (NAB) }\end{array}$ & $\begin{array}{l}\text { Prueba cronometrada de papel y lápiz } \\
\text { de siete laberintos de dificultad } \\
\text { creciente que mide la previsión y la } \\
\text { planificación. }\end{array}$ \\
\hline Cognición social & $\begin{array}{l}\text { Test de inteligencia } \\
\text { emocional Mayer-Salovery- } \\
\text { Carusso (MSCEIT): manejo } \\
\text { de emociones }\end{array}$ & $\begin{array}{l}\text { Test de selección múltiple que evalúa } \\
\text { cómo las personas manejan sus } \\
\text { emociones. }\end{array}$ \\
\hline
\end{tabular}

La evaluación de la cognición social se recomienda realizarse con la prueba de Inteligencia Emocional Mayer-Salovey-Caruso (Mayer, Salovery y Caruso, 2002; Extremera y Fernández-Berrocal, 2009). El MSCEIT (Mayer-Salovey-Caruso Emotional Intelligence Test) es una medida estandarizada del procesamiento de emociones que incluye las habilidades para percibir, facilitar el pensamiento, comprender y controlar dichas emociones (Mayer, Salovery y Caruso, 2003). Según el protocolo MATRICS se recomienda utilizar únicamente la rama de manejo de emociones, lo que a valoración personal puede limitar y dejar sin evaluar aspectos importantes de la cognición social como la percepción de emociones, la Teoría de la Mente o la empatía.

\subsubsection{Cerebro y neurocognición}

El deterioro de las funciones ejecutivas se ha atribuido a un funcionamiento anormal de la corteza prefrontal dorsolateral en pacientes psiquiátricos $\mathrm{y}$, en pacientes con esquizofrenia, este funcionamiento anómalo 
podría estar relacionado con déficits neuropsicológicos, especialmente en las funciones ejecutivas y en la memoria de trabajo (Palomares, Portella, Díaz-Marsá y López-Picó, 2019). Las regiones cerebrales asociadas con estas deficiencias en el funcionamiento cognitivo, la activación reducida en la corteza prefrontal dorsolateral se ha replicado consistentemente (Aleman, 2014). Sin embargo, varios estudios sugieren un aumento en la actividad prefrontal durante las tareas de memoria de trabajo que los pacientes aún son capaces de realizar. Pero, cuando la tarea se vuelve más difícil, su desempeño es peor que el de los sujetos controles y, típicamente, muestran menor activación (Aleman, 2014). Con respecto a la corteza temporal, se ha observado sistemáticamente su participación durante tareas que exploran la memoria episódica (Achim y Lepage, 2005).

Las anomalías en la corteza prefrontal dorsolateral, el hipocampo y la sustancia blanca se han asociado a un deterioro cognitivo global en la esquizofrenia; no obstante, Jirsaraie, Sheffield y Barch (2018) advierten que algunas de estas estructuras cerebrales también pueden estar específicamente relacionadas con déficits en dominios cognitivos concretos (principalmente, la función ejecutiva), más allá del déficit cognitivo global.

Se ha sugerido también que existe un mecanismo común que contribuye a los déficits cognitivos e incluye un deterioro en el control proactivo que puede influir en el rendimiento en una amplia variedad de dominios cognitivos (Barch y Ceaser, 2012). A nivel neural, un denominador común de dichos déficits parece ser la función alterada de la corteza prefrontal dorsolateral, su conectividad con otras áreas cerebrales y los sistemas de neurotransmisión que ayudan a la actualización y mantenimiento de las representaciones de objetivos que permiten el control proactivo (Barch y Ceaser, 2012). Así mismo, también se ha observado una relación entre los déficits neurocognitivos y una dinámica reducida en la llamada "red neural por defecto" (Kottaram et al., 2019).

Por otro lado, las alteraciones de la sustancia blanca también se han documentado en la esquizofrenia. En un reciente estudio mediante tractografía, 
Gómez-Gastiasoro et al. (2019) concluyeron que las anormalidades de la sustancia blanca podrían contribuir a una forma central de disfunción cognitiva que incluiría el procesamiento del lenguaje y la memoria episódica.

Respecto a la especificidad del perfil neurocognitivo en la esquizofrenia, los datos no son concluyentes. Varios estudios que compararon el funcionamiento neuropsicológico en pacientes con primeros episodios de esquizofrenia, trastorno bipolar y otros trastornos psicóticos encontraron diferencias significativas entre los grupos, siendo más graves en los pacientes con esquizofrenia (Jabben, Arts, Van Os y Krabbendam, 2010). Sin embargo, otras investigaciones mostraron un deterioro similar en la esquizofrenia y en el trastorno bipolar (Hill et al., 2009), y otros hallaron un funcionamiento cognitivo similar en el trastorno límite de la personalidad, la esquizofrenia y el trastorno bipolar (Lozano, Soriano, Aznarte, Gómez-Ariza y Bajo, 2016), lo que pondría en duda la supuesta especificidad del déficit cognitivo en la esquizofrenia. Recientemente, Palomares et al. (2019) compararon dos muestras de pacientes con un primer episodio psicótico o con trastorno límite de la personalidad a través de una amplia batería de evaluación neuropsicológica. Ambos grupos de pacientes mostraron un deterioro significativo de las funciones cognitivas en comparación con una muestra de controles sanos, pero no se diferenciaron entre ellos ni en el tipo ni en la gravedad del deterioro neuropsicológico. Estos resultados sugieren que los déficits neuropsicológicos podrían representar una diátesis patológica común e inespecífica, lo que llevaría a una desregulación clínica, emocional y comportamental en los trastornos mentales graves en general, más que en la esquizofrenia en particular (Palomares et al., 2019).

En suma, la investigación reciente señala que los déficits neurocognitivos, especialmente en la memoria verbal, podrían ser de gran importancia y tener mayor responsabilidad que los síntomas clínicos sobre el funcionamiento en la vida real (Lepage et al., 2014). Además, numerosos estudios muestran que el funcionamiento neuropsicológico, en particular la memoria verbal, la velocidad de procesamiento y las funciones ejecutivas, se ven alteradas incluso en las fases iniciales de la enfermedad (Ludwig, Pinkham, Harvey, Kelsven y Penn, 2017); que 
casi todos los pacientes con esquizofrenia se desempeñan por debajo de las expectativas en comparación con sus familiares no afectados (Keefe et al., 2006); y que las personas de alto riesgo rinden peor en memoria de trabajo en comparación con individuos sanos (Brüne et al., 2011). En pacientes jóvenes se ha encontrado que el volumen de la materia gris cerebelar se asocia con la función cognitiva y la psicopatología en la adolescencia (Moberget et al., 2019), y que el envejecimiento acelerado de estas redes cerebrales estaría a la base de la función cognitiva en los trastornos psicóticos (Sheffield, Rogers, Blackford, Heckers y Woodward, 2019). La integración funcional de las redes cognitivas de orden superior está intacta en la psicosis temprana, pero muestra signos de envejecimiento acelerado, lo que advierte del valor potencial de intervenciones dirigidas a la cognición durante las fases tempranas de la enfermedad (Sheffield et al., 2019).

Sin embargo, algunos autores han argumentado a favor de un modelo de neurodesarrollo frente a la clásica visión neurodegenerativa, incluso cómo los déficits neurocognitivos en la esquizofrenia se han podido sobredimensionar, atendiendo a que factores contextuales como la pobre motivación, la ansiedad de ejecución o la distracción debida a los propios síntomas durante las pruebas de evaluación, pudieran estar enmascarando los resultados, lo cual ha sido escasamente considerado en la mayoría de los estudios de investigación (Moritz et al., 2017). A nuestro parecer, se trata de una limitación importante. El rendimiento en las medidas neuropsicológicas podría deberse en gran medida a estos factores secundarios. En el estudio llevado a cabo por Moritz et al. (2017), la falta de motivación, los temores y las distracciones durante las pruebas, distinguieron a los pacientes de los controles y estas variables afectaron en gran medida al rendimiento. Por lo tanto, antes de concluir que los déficits neurocognitivos están presentes en los pacientes psiquiátricos, los clínicos deberían tener en cuenta estas influencias. Aunque las personas con esquizofrenia suelen, en promedio, obtener peores puntuaciones que los controles, un gran número de ellas muestra un rendimiento cognitivo preservado (Moritz et al., 2017). Por último, el impacto de la medicación antipsicótica en las 
redes neurales, en la morfología cerebral y en las capacidades cognitivas y emocionales está bien reconocida y cuenta con abundantes pruebas (Moncrieff, 2013; Jackson, 2008).

\subsection{La cognición social en la esquizofrenia}

La cognición social se define como la capacidad de las personas para pensar sobre sí mismas y sobre los demás en el contexto de las interacciones (Adolphs, 2009; Ochsner, 2008) e incluye funciones cognitivas que se emplean en situaciones socialmente relevantes (Harvey y Penn, 2010). Se trata de procesos que permiten a las personas comprender tanto las intenciones como las emociones de los demás y se relacionan con habilidades a menudo referidas como Teoría de la Mente (ToM, del inglés: Theory of Mind) e identificación del afecto (Brüne, 2005; Brüne, Schaub, Juckel y Langdon, 2011; Penn, Sanna y Roberts, 2008).

Tabla 6. Dominios de la cognición social

\begin{tabular}{ll}
\hline Teoría de la Mente & Habilidad para inferir las interacciones y creencias de los otros. \\
\hline Percepción social & $\begin{array}{l}\text { Habilidad para identificar roles sociales, reglas sociales y } \\
\text { contexto social. }\end{array}$ \\
\hline Conocimiento social & $\begin{array}{l}\text { Conciencia o conocimiento de los roles, reglas y objetivos que } \\
\text { caracterizan las situaciones sociales. }\end{array}$ \\
\hline Sesgo de atribución & $\begin{array}{l}\text { Cómo las personas infieren las causas de eventos concretos } \\
\text { positivos o negativos. }\end{array}$ \\
\hline Pensamiento emocional & $\begin{array}{l}\text { Cómo las personas identifican, facilitan, entienden y manejan las } \\
\text { emociones: } \\
\text { - percepción emocional }\end{array}$ \\
& $\begin{array}{l}\text { - facilitación emocional } \\
\text { comprensión emocional }\end{array}$ \\
& manejo emocional \\
\hline
\end{tabular}

Se reconocen cinco dominios de la cognición social (CS) (Tabla 6) que son: a) la teoría de la mente, b) la percepción social, c) el conocimiento social, d) el estilo atribucional y e) el procesamiento emocional (Rodríguez-Jiménez et al., 2012b). En líneas generales, la cognición social engloba la percepción emocional, 
la regulación emocional, la percepción social, la mentalización y el estilo atributivo, así como otras complejas capacidades sociales como la empatía (Pinkham, Harvey y Penn, 2017). No obstante, las personas con trastorno mental grave podrían no mostrar un déficit generalizado de la empatía, aunque sí que lo tendrían a la hora de reconocer estados emocionales mediante la mirada, lo que indicaría un procesamiento de la información para las caras diferente a los sujetos de la población normal (Martín-Cantero, Secades-Villa, Aparicio-Migueza y Tirapu-Ustárroz, 2017).

Mancuso et al. (Mancuso, Horan, Kern y Green, 2011) identificaron tres factores que integran elementos de los dominios anteriores: estilo atribucional; detección de señales sociales de nivel inferior; y un nivel superior de procesamiento inferencial. En la misma línea, Browne et al. (Browne et al., 2016) identifican un único factor que agrupa medidas de reconocimiento de emociones y de ToM (no incluyeron el estilo atribucional), y que se obtiene tanto en personas con psicosis como en sujetos sanos. Cuando se compara el rendimiento de pacientes frente a población general, se obtiene una estructura factorial diferente para cada grupo (Mancuso et al., 2011): dos factores en población con esquizofrenia y tres factores en población control. En esquizofrenia serían: a) estilo atribucional y b) habilidades de CS (incluyen percepción de emociones y ToM). En población normal coinciden con la solución factorial encontrada por Mancuso et al. (Mancuso et al., 2011) para pacientes: a) estilo atribucional, b) detección de señales sociales de nivel inferior y c) nivel superior de procesamiento inferencial.

\subsubsection{Procesos de cognición social en la esquizofrenia}

Green et al. (2015) incluyeron varios dominios del procesamiento social que se han investigado en personas con esquizofrenia: a) percepción de señales sociales (incluida la percepción de caras y voces); b) intercambio de experiencias (incluida la resonancia motora y el intercambio de afecto); c) mentalización, y d) experiencia y regulación emocional. 
Estos procesos pueden dividirse conforme al grado de deterioro. Cuatro de estos procesos se encuentran consistentemente deteriorados: la percepción de caras, la percepción de la voz, la mentalización y la regulación de emociones. Uno, la experiencia emocional, se encuentra frecuentemente intacto. Y los resultados son inconsistentes para dos subprocesos: la resonancia motora y el intercambio de afecto, pudiendo estar en gran parte intactos o solo sutilmente dañados (Figura 2). Los procesos deteriorados se consideran "procesos sociales reflejos", requieren mayor esfuerzo en el control del proceso, mientras que los procesos relativamente intactos se consideran "procesos sociales reflexivos", requieren menor esfuerzo que otros procesos sociales (Green et al., 2015).

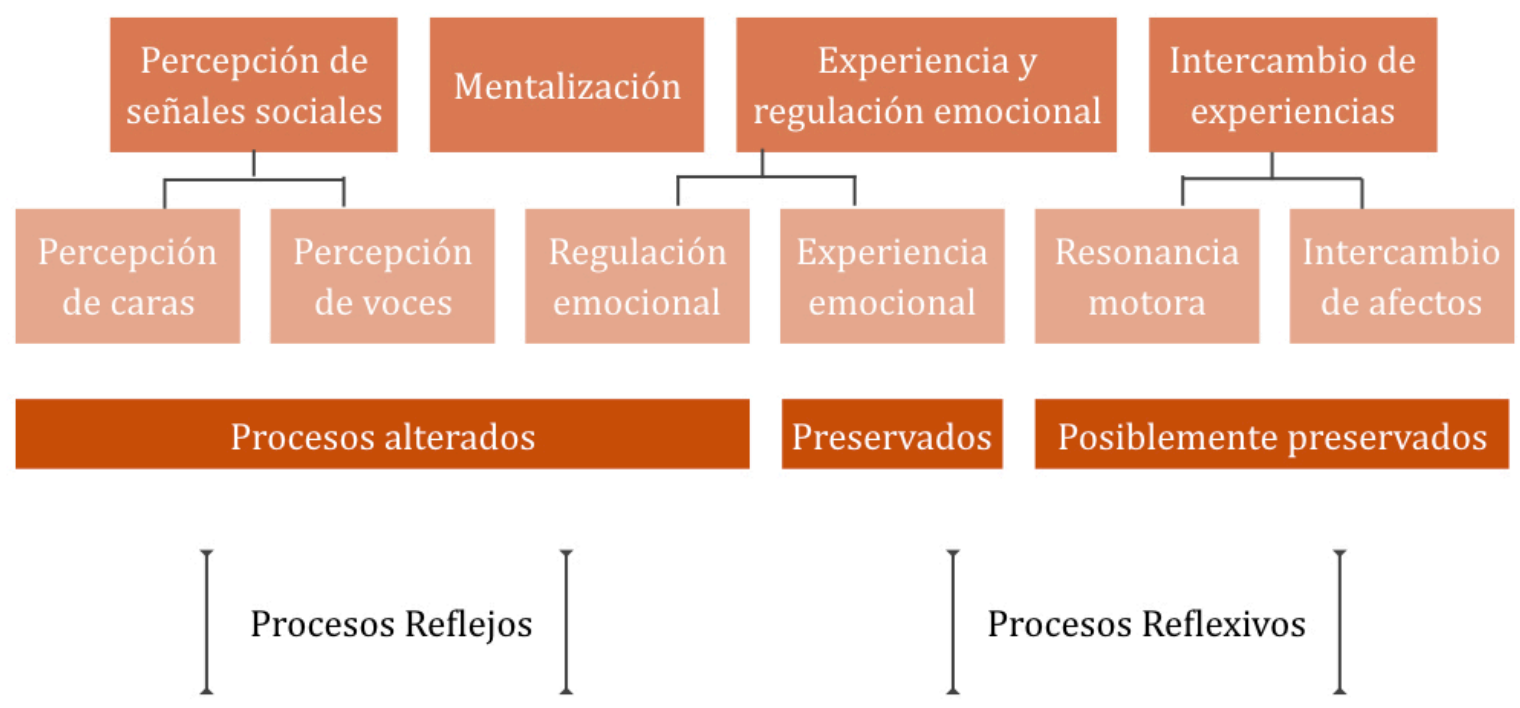

Figura 2. Procesos de cognición social alterados y preservados en la esquizofrenia

En relación con todo ello, se supone la existencia de distintos grados de deterioro cognitivo social en la esquizofrenia, por lo que los esfuerzos de recuperación pueden no ser necesarios para todos los individuos. Los resultados actuales demuestran que aproximadamente una cuarta parte de los pacientes no muestran deterioro (Hajdúk, Harvey, Penn y Pinkham, 2018), lo que cuestionaría la consideración del déficit en cognición social como un marcador generalizado de la esquizofrenia.

Finalmente, cabe destacar que varios modelos psicológicos integrados sobre la psicosis han vinculado el contexto, las experiencias adversas y los 
procesos afectivos y cognitivos al inicio de la psicosis. En estos modelos, los efectos del estrés contribuyen al desarrollo de las experiencias psicóticas a través de perturbaciones afectivas, sesgos cognitivos y experiencias anómalas (Klippel et al., 2017).

Los modelos psicológicos de la psicosis proporcionan una especificación detallada de los procesos cognitivos asociados con las respuestas emocionales al estrés y consideran algunos sesgos cognitivos, como la hipervigilancia, implicados en la reactividad al estrés. De este modo, atribuyen un papel destacado a las dificultades afectivas inducidas por el estrés (p.ej., los síntomas de ansiedad) que impulsarían a las personas a una mayor "anticipación de la amenaza" y, a su vez, esa mayor anticipación de amenaza estaría vinculada a la formación de las experiencias psicóticas. Dado que las personas intentan dar significado a tales experiencias, los sesgos en procesos cognitivos resultan en la valoración de estas experiencias anómalas como incontrolables, atemorizantes, atribuibles a causas "externas" y, finalmente, conducen a creencias extrañas (Howes y Murray, 2014; van Os y Linscott, 2012).

Los modelos cognitivos basados en enfoques neurobiológicos sostienen que estas experiencias están estrechamente vinculadas a un sistema dopaminérgico sensibilizado, secundario a variantes genéticas, a daños neurológicos tempranos y a la exposición a acontecimientos sociales adversos (Garety, Bebbington, Fowler, Freeman y Kuipers, 2007).

De esta manera, la interacción entre los sesgos cognitivos, las perturbaciones afectivas y el apego inseguro podría ser de una importancia excepcional en el inicio de las experiencias psicóticas (Sheinbaum et al., 2015).

\subsubsection{Evaluación de la cognición social}

La CS se reconoce como un importante objetivo en el tratamiento de la esquizofrenia. No obstante, la escasez de medidas bien validadas para el uso en ensayos clínicos sigue siendo una importante limitación. El estudio SCOPE (del inglés: Social Cognition Psychometric Evaluation) abordó esta limitación mediante 
la evaluación sistemática de las propiedades psicométricas de instrumentos de medida prometedores (Pinkham et al., 2014). Los resultados obtenidos en las distintas fases del estudio SCOPE recomiendan los instrumentos Hinting Task (Test de las insinuaciones) (Corcoran, Mercer y Frith, 1995), ER-40 (Penn Emotion Recognition Task) (Kohler et al., 2003) y BLERT (Bell Lysaker Emotion Recognition Task) (Bryson, Bell y Lysaker, 1997) dirigidos a la evaluación de la CS. Estas tareas juntas evalúan los dominios del procesamiento de las emociones y la atribución del estado mental. No obstante, no se recomienda el uso de estas tareas para abordar en concreto la percepción social o el estilo atribucional. Por otro lado, se considera que puede tener utilidad el Cuestionario de Intenciones Hostiles Ambiguas (Ambiguous Intentions Hostility Questionnaire) (Combs, Penn, Wicher y Waldheter, 2007). Estos tres instrumentos mostraron propiedades psicométricas más fuertes y se recomiendan para su uso en ensayos clínicos. Otros instrumentos evaluados mostraron propiedades psicométricas más pobres que sugieren tener precaución a la hora de utilizarlos (Pinkham et al., 2017).

En suma, los autores hacen hincapié sobre la necesidad de seguir desarrollando nuevas medidas para generar una batería más fiable y válida para la evaluación de la CS, puesto que el estudio identificó más medidas que no deberían usarse (o usarse con precaución) que instrumentos que sí deberían usarse. Además, la escasa validez ecológica de las medidas habitualmente utilizadas aparece como otra importante limitación.

\subsubsection{Bases neuroanatómicas asociadas a los procesos sociales}

Hace años, se sugirió que las bases neurales de la CS en la esquizofrenia residían en un conjunto de estructuras que constituirían el denominado "Cerebro Social". Éste incluía áreas cerebrales distribuidas a través de regiones frontales, parietales y temporales que se integran en una red extensa asociada con varios aspectos de la cognición. El córtex prefrontal medial, el córtex cingulado anterior, la unión temporoparietal, el córtex superior temporal posterior y el polo temporal se convertían en el núcleo del cerebro social implicado en la CS y se 
asociaban a algunas anomalías en la ToM, la percepción de emociones y la capacidad para considerar la perspectiva de los otros, fenómenos comúnmente encontrados en la esquizofrenia (García et al., 2018).

Estudios recientes proponen nuevos modelos neuroanatómicos y cognitivos que relacionan un conjunto de áreas del cerebro, en su mayoría componentes del cerebro social, con varios aspectos de la CS. Sobre la base de estos modelos, Billeke y Aboitiz (2013) afirman que las personas con esquizofrenia tendrán un alto grado de deterioro en conductas como el procesamiento de emociones o la comunicación de intenciones.

Además, las técnicas de neuroimagen proporcionan algunas evidencias de un deterioro significativo en regiones como la amígdala, el giro cingulado anterior y la corteza prefrontal medial, lo que establecería una base estructural para el deterioro que se ha observado en pacientes con esquizofrenia en el procesamiento emocional y la ToM, respectivamente (García et al., 2018).

La cognición social se puede dividir en varios procesos distintos que involucran regiones cerebrales diferentes, habiendo cierta superposición entre procesos en algunas de estas áreas (Green et al., 2015) (Figura 3):

A) La percepción de señales sociales incluye, por un lado, la percepción de caras, que se asocia con la actividad de la amígdala y el área fusiforme facial (FFA) y, por otro lado, la percepción de la voz, que activa el giro temporal superior y el giro frontal inferior.

B) El intercambio de experiencias incluye los procesos de resonancia motora, que activan el lóbulo parietal inferior y la corteza premotora, así como el intercambio de afectos, donde se activan la corteza cingulada anterior dorsal y la ínsula anterior.

C) La mentalización se relaciona con varias áreas, incluyendo la unión temporoparietal (JPT), el lóbulo temporal, el precuneus y el córtex prefrontal medial (CPFm).

D) En la experiencia emocional se activan la amígdala, el hipocampo anterior (no se muestra en la figura), la ínsula anterior y la corteza cingulada anterior. La 
regulación de emociones activa el córtex prefrontal dorsolateral, el córtex prefrontal ventrolateral (CPFvl) y la amígdala.

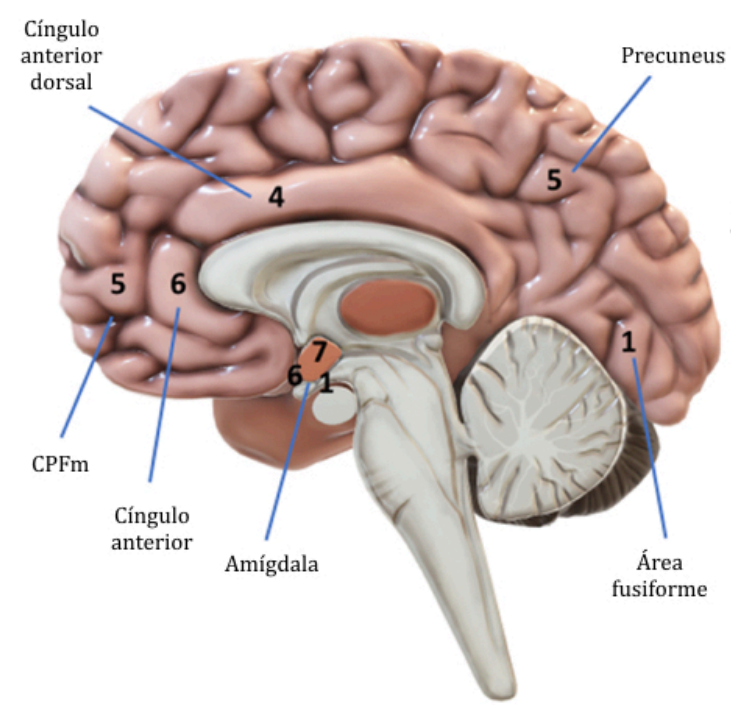

1. Percepción facial

2. Percepción de voces

\begin{abstract}
3. Resonancia motora
\end{abstract}
4. Afecto compartido

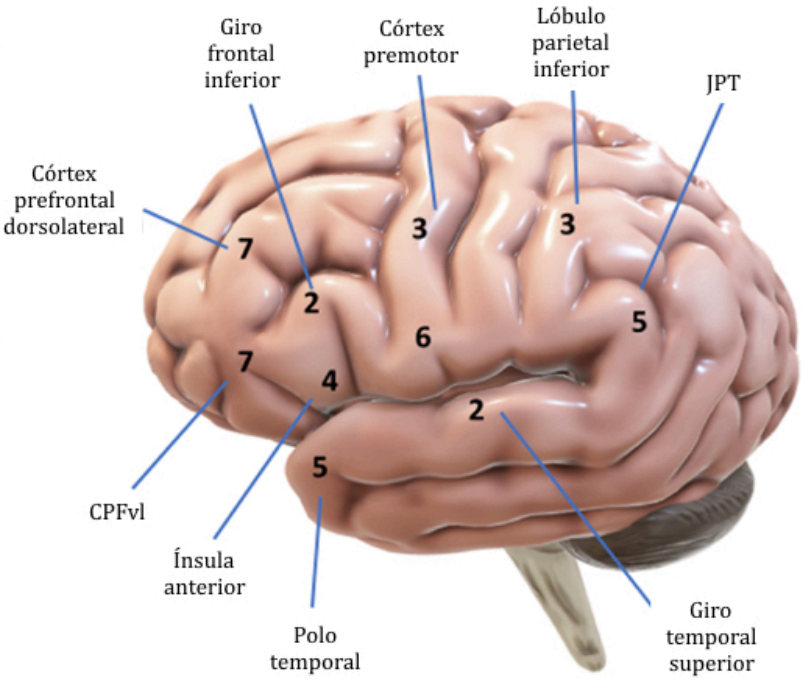

5. Mentalización

6. Experiencia emocional

7. Regulación emocional

Figura 3. Regiones cerebrales relacionadas con diferentes áreas de la cognición social

Estas regiones cerebrales y los procesos sociales asociados no están completamente separados; por ejemplo, la ínsula anterior está involucrada tanto en el intercambio de afectos como en la experiencia emocional y la amígdala participa en la percepción de caras y la experiencia y regulación emocionales. Es importante entender que estas áreas son representativas, pero no exhaustivas, de las áreas cerebrales consideradas relevantes para cada proceso cognitivo social (Green et al., 2015).

Los déficits cognitivo-sociales se han llegado a considerar marcadores de vulnerabilidad para la esquizofrenia y otros trastornos del espectro psicótico, y podrían ser determinantes en la recuperación, incluyendo las relaciones interpersonales, el éxito educativo y los resultados vocacionales (Javed y Charles, 2018). No obstante, a pesar de la evidencia, todavía no podemos establecer firmemente que la cognición social sea, más allá de una observación comúnmente aceptada, un marcador endofenotípico de la psicosis (al menos, no de todas sus 
formas) y da cuenta, tan solo, de una pequeña parte de la varianza de los resultados a largo plazo en lo referente a la recuperación (Beck, Himelstein, Bredemeier, Silverstein y Grant, 2018; Jobe y Harrow, 2010).

Finalmente, cabe también destacar un amplio estudio longitudinal llevado a cabo por Jobe y Harrow (2010) durante un periodo de 26 años. En él se estudiaron varios factores de riesgo relacionados con el estrés o con la vulnerabilidad a la psicosis que podrían explicar el curso episódico que experimentan la mayoría de las personas con esquizofrenia. Estos factores interactúan con la personalidad, el temperamento y los sesgos cognitivos que, si bien no causan la psicosis, influyen en su evolución. La investigación se centró, entre otros, en factores como la ansiedad-rasgo, un pobre desarrollo psicosocial antes de la enfermedad, rasgos de personalidad como el locus de control, estilos cognitivos, el nivel de deterioro neurocognitivo y la duración de la psicosis no tratada. Además, resulta interesante cómo el mencionado estudio encontró que, a pesar de la eficacia probada de los fármacos antipsicóticos a corto plazo, hubo un subgrupo de pacientes con esquizofrenia que, pocos años después de la fase aguda, mantenían un buen funcionamiento o experimentaban períodos largos de recuperación, sin tratamiento neuroléptico (Harrow, Jobe, Grossman y Faull, 2015). Este estudio representa un ejemplo paradigmático de cómo enfocar el estudio de la influencia de las variables revisadas en este capítulo, en la recuperación a largo plazo de las personas con psicosis. 


\section{Capítulo 4 \\ Cognición en la esquizofrenia (II) ${ }^{2}$}

Sesgos cognitivos, modelos explicativos

y programas de intervención

\subsection{Introducción}

A pesar de que los déficits neurocognitivos y de cognición social no se incluyen en el diagnóstico de esquizofrenia de la clasificación DSM-5 (APA, 2013), es clara su importancia para el diagnóstico, la recuperación y el funcionamiento diario del paciente. El deterioro cognitivo sí se incluye como especificador diagnóstico en la clasificación diagnóstica CIE-11 (OMS, 2018) y, además, está comúnmente aceptado que los diferentes dominios de la cognición afectan de algún modo al funcionamiento psicosocial de las personas con esquizofrenia (Green et al., 2008; Green, Olivier, Crawley, Penn y Silverstein, 2005; Schmidt et al., 2011).

En el anterior capítulo, se intentó dar cuenta del estado de la cuestión en lo referente a los diferentes aspectos de la cognición, de su evaluación, de las bases neurales de dichos procesos, y de sus implicaciones en el funcionamiento social de las personas con esquizofrenia En el presente capítulo se describen los diferentes sesgos cognitivos que parecen caracterizar a la psicosis. Asimismo, se dará cuenta de los diferentes modelos que relacionan los distintos dominios de la neurocognición, la cognición social y la metacognición, y su vínculo con los síntomas y el funcionamiento social. Finalmente, se consideran algunos de los programas de intervención que han demostrado eficacia en la mejora de la

\footnotetext{
2El presente capítulo ha sido publicado como artículo de revisión en:

Pena-Garijo, J., \& Monfort-Escrig, C. (2020). Cognition in schizophrenia. State of the art (Par II): cognitive biases, explanatory models, and intervention programs. Revista Asociación Española de Neuropsiquiatría, 40, 131-154. Disponible en: http://www.revistaaen.es/index.php/aen/article/ view/17122
} 
cognición en la esquizofrenia y las bases neurales que podrían sustentar el cambio.

\subsection{Sesgos cognitivos y sintomatología en la esquizofrenia}

\subsubsection{Sesgos de razonamiento en las psicosis}

La metacognición se puede definir como el proceso de "pensar acerca de la forma en que pensamos", por lo que resulta de gran relevancia como herramienta de intervención cognitiva en el manejo de los sesgos de razonamiento y problemas en la cognición social. Según Flavell (1979) se trata de un conocimiento adquirido para manejar y actuar sobre los propios procesos cognitivos, lo que permite reconocer limitaciones, analizar problemas y crear nuevas estrategias para resolverlos. El autor distingue cuatro componentes que interactúan de manera compleja: a) conocimiento metacognitivo, b) experiencias metacognitivas, c) metas (o tareas) metacognitivas, y d) acciones metacognitivas (Moritz y Lysaker, 2018).

Según un modelo integrador, la metacognición se refiere a un espectro de actividades mentales que pueden ir desde procesos discretos que involucran notar pensamientos y sentimientos específicos, a actos más sintéticos en los que la información sobre uno mismo y sobre los otros se integra en representaciones complejas que permiten a las personas reconocer y responder a los desafíos de la experiencia cotidiana (Lysaker y Dimaggio, 2014; Lysar y Hasson-Ohayon, 2014)). Los procesos metacognitivos se conceptualizan como procesos que permiten que surja un sentido coherente del yo y de los otros dentro del flujo de la vida (Lysaker et al. 2018). Así, desde una perspectiva fenomenológica, algunos estudiosos consideran la esquizofrenia como un trastorno del yo más que del cerebro (...), rechazando la visión cerebrocentrista que se impone desde la psiquiatría más biológica y entendiendo a la persona en su contexto histórico, interpersonal y social. 
La relación entre los sesgos cognitivos y la esquizofrenia se encuentra ampliamente fundamentada en la literatura científica. En este sentido, los sesgos cognitivos serían un factor de desviación en el juicio, donde las inferencias que hacemos sobre otras personas y/o situaciones pueden ser ilógicas (Prike, Arnold y Williamson, 2018).

Desde una óptica dimensional, la "hipótesis de la continuidad" (van Os y Reininghaus, 2016) afirma que la psicosis no es un constructo dicotómico (salud vs. psicosis), sino que incluye una amplia gama de fenómenos que varían en términos de frecuencia, intensidad, grado de angustia o necesidad de ayuda médica que requieren. Así, se ha demostrado un aumento de los sesgos cognitivos en sujetos con síntomas subclínicos de paranoia (Klein, Kelsven y Pinkham, 2018).

Los modelos psicológicos que explican la formación de creencias extrañas ya fueron descritos en el capítulo anterior. Estos modelos han vinculado el contexto, las experiencias adversas y los procesos afectivos y cognitivos al inicio de la psicosis. Además, asociaron los efectos del estrés con el desarrollo de las experiencias psicóticas a través de perturbaciones afectivas, sesgos cognitivos y experiencias anómalas (Howes y Murray, 2014; Klippel et al., 2017; van Os y Linscott, 2012).

Numerosas investigaciones ponen de relieve la importancia de un sesgo de razonamiento cognitivo denominado "salto a conclusiones" (JTC, del inglés: jumping to conclusions) por su influencia en la formación y mantenimiento de los delirios (Moritz, Woodward y Lambert, 2007; Moritz y Woodward, 2005). Describe un estilo de razonamiento caracterizado por una recopilación de información limitada para tomar decisiones, por lo que se tiende a tomar decisiones apresuradas en base a una evidencia incompleta. Está presente en pacientes no delirantes con esquizofrenia, en pacientes con delirios en remisión, en familiares de primer grado de individuos psicóticos (Moritz y Woodward, 2005), en personas con experiencias psicóticas (Gawęda et al., 2018) y es capaz 
de predecir las fluctuaciones en los síntomas paranoicos a lo largo del día (Lüdtke, Kriston, Schröder, Lincoln y Moritz, 2017).

Relacionado con el JTC, en las personas con esquizofrenia existe también una disminución del umbral de confianza sobre sus decisiones que, finalmente, acaban tomando con poca evidencia (Moritz et al., 2016). Algunas hipótesis vinculan este sesgo con la motivación y el afecto, mientras que otras consideran que los mecanismos subyacentes tienen que ver con los déficits neurocognitivos (Fine, Gardner, Craigie y Gold, 2007; Freeman, 2007; Moritz, Woodward, Jelinek y Klinge, 2008; Lincoln, Peter, Schäfer y Moritz, 2010; Lunt et al., 2012).

Además, estos sesgos están asociados con la existencia de delirios en múltiples diagnósticos; esto es, no son patognomónicos del diagnóstico de esquizofrenia. Sin embargo, esta asociación es capaz de explicar (al menos, en parte) la severidad de los delirios, su persistencia en el tiempo, su grado de convicción, su desarrollo y su mantenimiento durante las fases agudas de la enfermedad (McLean, Mattiske y Balzan, 2017).

También se ha explorado la posible relación del sesgo JTC con el sesgo contra la evidencia no confirmatoria (BADE, del inglés: bias against disconfirmatory evidence) y el sesgo contra la evidencia confirmatoria (BACE, del inglés: bias against confirmatory evidence). El BADE es un sesgo cognitivo donde, independientemente de la información inconsistente, la hipótesis se sostiene a pesar de la evidencia contraria. La investigación sugiere que este sesgo se extiende más allá del delirio en sí mismo y contribuye a su persistencia al disminuir la probabilidad de que las creencias delirantes se vuelvan a evaluar cuando se proporcionan pruebas en contra (McLean et al., 2017). Por otro lado, en el BACE, los sujetos, independientemente de que la información sea inconsistente, mantienen su creencia o hipótesis debido a la evidencia a favor de esta (Moritz, Woodward y Hausmann, 2006; Júarez et al., 2014; Buchy, Woodward y Liotti, 2007).

También el estilo atribucional ha sido repetidamente relacionado con la presencia de sintomatología positiva en la esquizofrenia. Bentall et al. (Bentall, 
Corcoran, Howard, Blackwood y Kinderman, 2001) diferencian dos sesgos: una tendencia general a hacer atribuciones "no-propias", incluyendo a otras personas y a las circunstancias, para los eventos positivos (sesgo externalizante), y una tendencia específica a culpar a los demás por los eventos negativos (sesgo personalizador). Las personas que tienden a explicar eventos perturbadores en términos de causas externas (no-propias) pueden ser particularmente propensas a desarrollar creencias delirantes sobre tales experiencias (Langdon, Still, Connors, Ward y Catts, 2013). Por otro lado, varios estudios observaron una mayor tendencia a atribuir eventos positivos (pero no negativos) a uno mismo (So, Tang y Leung, 2015). Además, se ha hipotetizado que los delirios persecutorios causados por un sesgo atributivo externo ayudan a mantener preservada la autoestima en las personas delirantes (Murphy, Bentall, Freeman, O'Rourke y Hutton, 2018). Sin embargo, investigaciones recientes desde este "modelo de defensa" de los delirios persecutorios no sustentan la idea inicial y, por el contrario, predicen que las personas con este tipo de delirios tienen una autoestima más baja y un mayor sesgo externalizante que las personas con depresión (Murphy et al., 2018).

En todo caso, la relación entre los estilos atribucionales y los síntomas psicóticos ha sido explicada satisfactoriamente por modelos firmemente establecidos en el campo de estudio (Bentall et al., 2001; Murphy et al., 2018; Garety y Freeman, 2013; Moritz, Bentall, Kolbeck y Roesch-Ely, 2018). Por último, se ha sugerido que los individuos con psicosis establecen atribuciones "monocausales" y que éstas podrían jugar un papel importante en la patogénesis o, incluso, representar un factor de vulnerabilidad para la psicosis (Moritz et al., 2018; Randjbar, Veckenstedt, Vitzhum, Hottenrott y Moritz, 2011).

Otros sesgos de razonamiento se han estudiado con menor profundidad. Por ejemplo, en el sesgo de sobreconfianza (overconfidence bias) las personas con esquizofrenia muestran mayores juicios erróneos respecto a la confiabilidad de su propia respuesta. No obstante, otros estudios han obtenido datos menos significativos, así como datos similares en pacientes con otros trastornos, como el 
trastorno obsesivo-compulsivo (Juárez-Ramos et al., 2014; Moritz, Thompson y Andreou, 2014).

Además, algunos trabajos han investigado si el apego inseguro y los sesgos cognitivos operan como mediadores entre eventos traumáticos y experiencias psicóticas en muestras no clínicas (Gawęda et al., 2018).

\subsubsection{Neurocognición y sesgo de salto a conclusiones}

Los modelos cognitivos postulan que los efectos del estrés contribuyen al desarrollo de las experiencias psicóticas a través de perturbaciones afectivas y sesgos cognitivos. Las implicaciones neurobiológicas de estos modelos apuntan a que las experiencias psicóticas están estrechamente vinculadas a un sistema dopaminérgico sensibilizado, secundario a variantes genéticas, a daños neurológicos tempranos y a la exposición a acontecimientos sociales adversos (Garety et al., 2007).

Se ha encontrado que las personas que saltan a conclusiones tienen un funcionamiento deficiente en tareas de memoria de trabajo y memoria verbal (González et al., 2018). A su vez, tanto la neurocognición como la CS podrían estar involucradas en la formación de los delirios mediante la influencia del sesgo JTC y la confianza en la toma de decisiones (Takeda et al., 2018), lo que enlaza la disfunción neurocognitiva con los sesgos de razonamiento para explicar la formación de los síntomas psicóticos.

La evaluación del funcionamiento cognitivo en personas con esquizofrenia muestra una relación del JTC con la memoria de trabajo, la memoria verbal y la velocidad de procesamiento (Ochoa et al., 2014). Además, diversas intervenciones como la terapia de rehabilitación cognitiva y el entrenamiento metacognitivo (MCT, del inglés: Metacognitive Training) podrían mejorar la memoria y reducir el sesgo JTC (Penadés et al., 2010; Moritz et al., 2011). Por último, se ha observado que el JTC está presente incluso en etapas tempranas de la enfermedad y se ha relacionado con el funcionamiento neuropsicológico (González et al., 2018). 


\subsection{Relaciones entre neurocognición, cognición social, metacognición y funcionamiento social}

\subsubsection{Modelos explicativos}

La relación entre neurocognición y cognición social se ha convertido en un área de interés dentro del estudio de la esquizofrenia. Diferentes modelos se han propuesto para explicar estas relaciones.

El modelo de Vauth et al. (Vauth, Rüsch, Wirtz y Corrigan, 2004) hipotetiza que la cognición social es un mediador entre la neurocognición básica y el funcionamiento social. En la Figura 4 se muestran los valores que reflejan la influencia de unos factores sobre otros (Rebolleda, 2017).

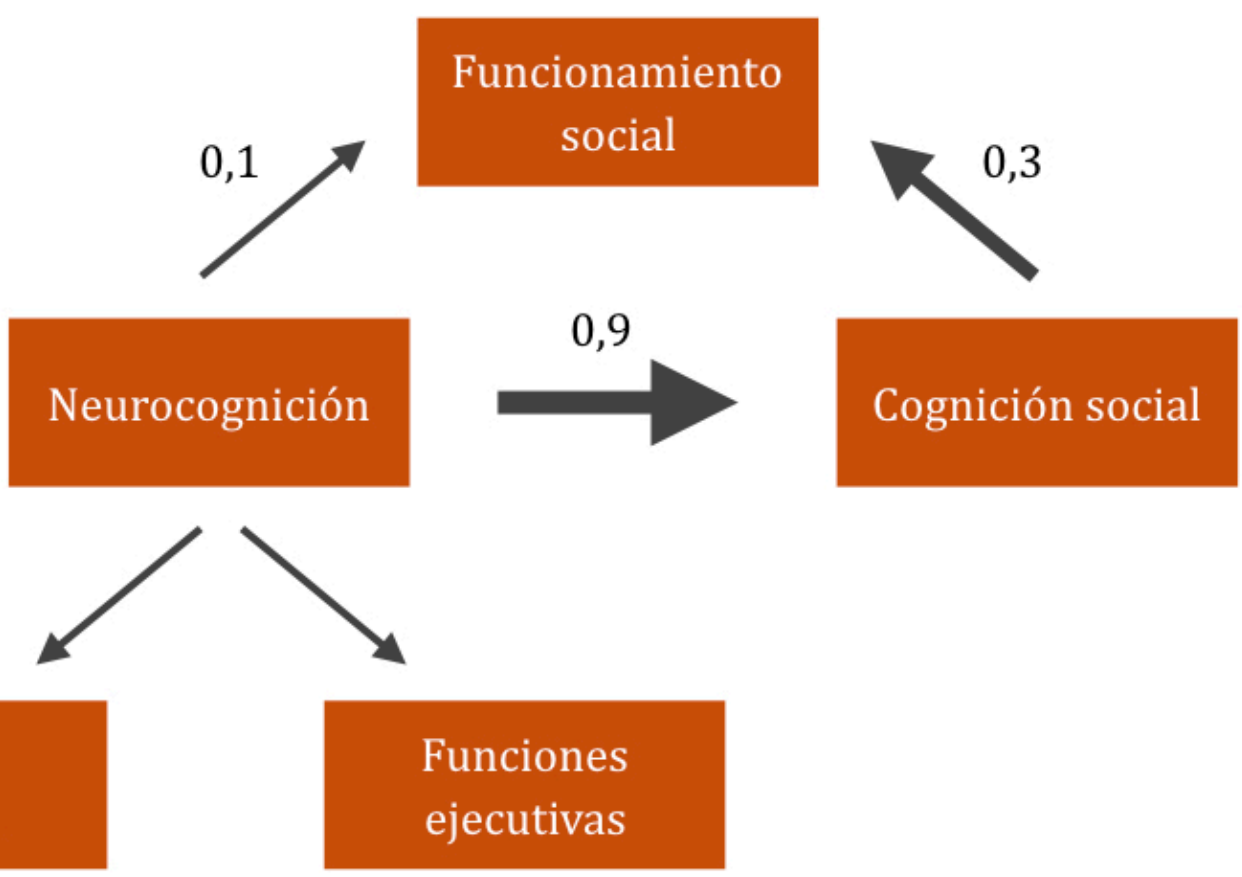

Figura 4. Modelo de Vauth et al. Relaciones entre cognición y funcionamiento social en la esquizofrenia

El modelo de Brekke et al. (Brekke, Kay, Lee y Green, 2005) es un modelo biosocial causal del funcionamiento social en la esquizofrenia (Figura 5). La neurocognición, la CS, la competencia social y el soporte social actúan como predictores del funcionamiento social global, siendo el grosor de las líneas proporcional a la influencia de unos factores sobre otros (Rebolleda, 2017). 


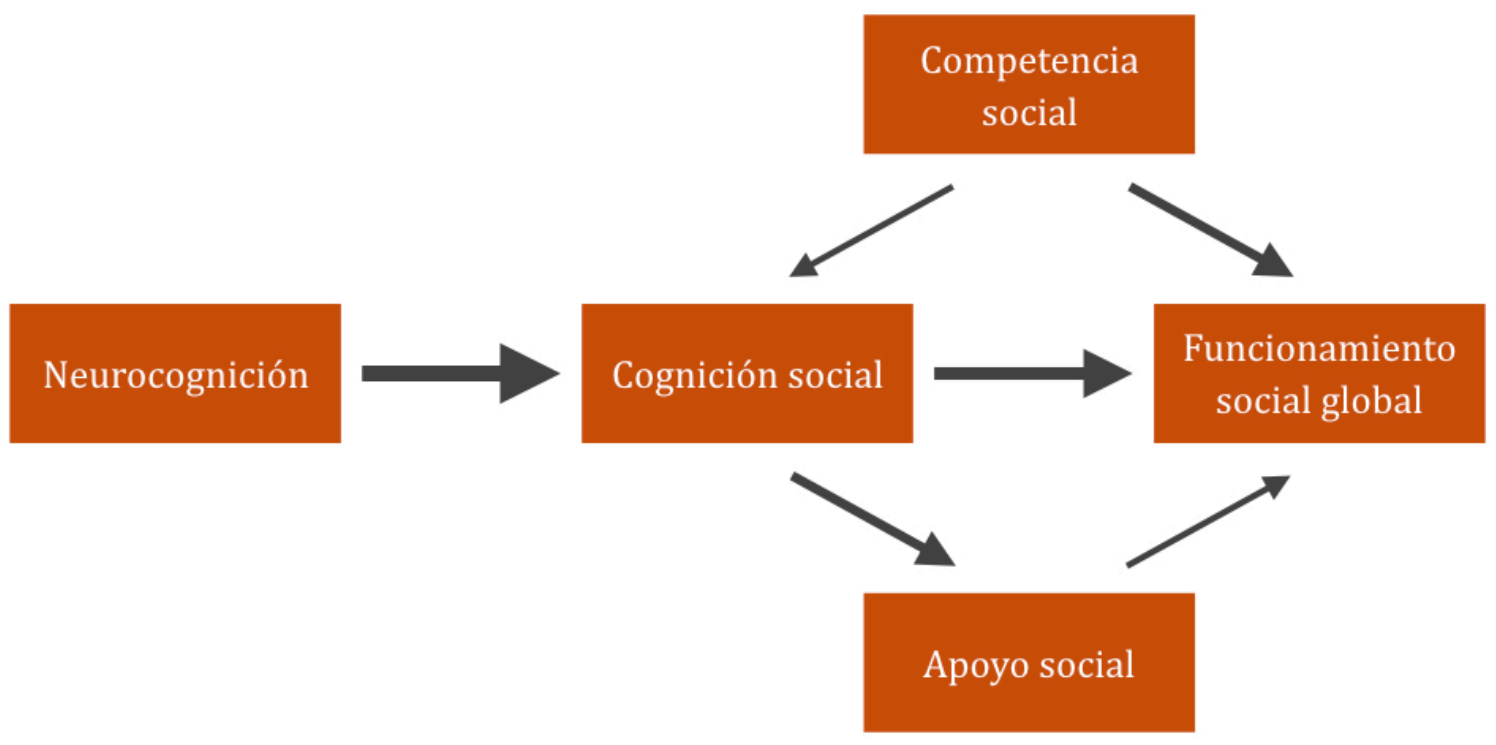

Figura 5. Modelo biopsicosocial de Brekke et al. La neurocognición explica el funcionamiento social global a través de la cognición social

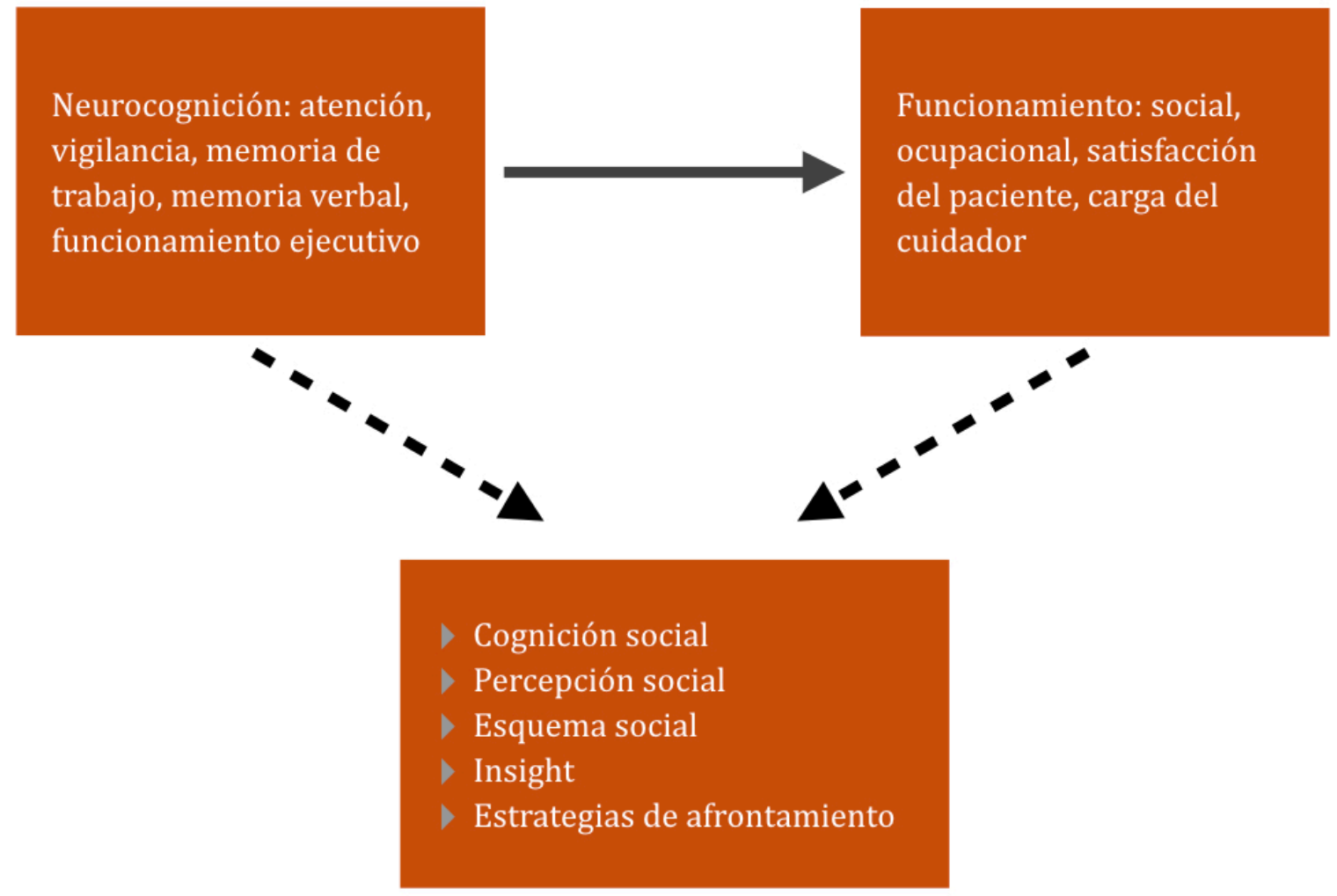

Figura 6. Modelo de Green y Nuechterlein. Las asociaciones entre la cognición social y la neurocognición se presentan como potenciales predictores del funcionamiento social. 
El modelo de Green y Nuechterlein (1999) es un modelo complejo que presenta por separado los subcomponentes de la neurocognición, la CS y el funcionamiento psicosocial (Figura 6). En este modelo, las asociaciones entre la CS y la neurocognición se presentan como potenciales predictores del funcionamiento social (Rebolleda, 2017).

Más recientemente, Green et al. (2018) proponen un modelo que se enfoca en un aspecto funcional de la discapacidad social en la esquizofrenia, al que denominan "desconexión social". Parte de dos ámbitos de la investigación: la discapacidad social en la esquizofrenia y la desconexión social en la comunidad. Ambas líneas de investigación han actuado en paralelo con escasa interacción entre ellas. Es posible que la desconexión social en la comunidad y el espectro de las psicosis compartan factores de riesgo o que la desconexión social sea un factor de riesgo para el desarrollo de la esquizofrenia. En cualquier caso, la desconexión social es una condición general que supone un problema de salud pública y que se debe considerar, tanto entre los trastornos psiquiátricos como en otras condiciones de salud.

Además, diferentes autores han encontrado que, desde el inicio de los síntomas, los déficits en cognición social son independientes del Cociente Intelectual o del funcionamiento cognitivo (Fett et al., 2011; Mancuso et al., 2011), y varios análisis factoriales han confirmado que la CS y la neurocognición son dominios independientes (Sergi et al., 2007; van Hooren et al., 2008).

Por último, un reciente trabajo (Hasson-Ohayon, Goldzweig, LaviRotenberg, Luther y Lysaker, 2018) formula un modelo de red en el que se relacionan los síntomas (positivos, negativos, desorganización, hostilidad y malestar emocional), con la neurocognición, la CS y la metacognición. Los resultados sugieren que el nodo representado por los síntomas cognitivos es el más central en la red. Las habilidades de metacognición tienen las medidas de centralidad más fuertes, seguidas por el aprendizaje visual y la identificación de emociones. Estos resultados apoyan también la necesidad de integrar la recuperación cognitiva en el tratamiento y están en la línea de las terapias que se 
orientan a la intervención sobre los procesos de CS y en el entrenamiento metacognitivo de la esquizofrenia (Moritz et al., 2006; Horan y Green, 2019).

\subsubsection{Cognición y funcionamiento social}

La cognición social, en general, podría tener más peso que la neurocognición como predictor del funcionamiento diario de los pacientes. Los déficits en Teoría de la Mente (ToM) se han sugerido ampliamente como un buen predictor de los síntomas psicóticos en personas con trastornos del espectro de la esquizofrenia (Green y Nuechterlein, 1999; Green et al., 2018; Brown, Tan, Can, Esen-Danaci y Brüne, 2014; Fretland et al., 2015; Pickup y Frith, 2001; Rominger et al., 2016; Vaskinn, Andersson, Østefjells, Andreassen y Sundet, 2018) y se han propuesto también como un posible predictor de la recuperación (Ventura et al., 2015).

Al analizar tanto las variables clínicas como las relacionadas con la cognición, los síntomas negativos y la CS parecen ser los dos aspectos que más influyen en el funcionamiento social de las personas con esquizofrenia (Kalin et al., 2015). Incluso niveles bajos de síntomas negativos mostraron correlación con el funcionamiento social. Factores como la abulia, actitudes disfuncionales, el esfuerzo, el estrés, emociones negativas y los síntomas desorganizados explican un porcentaje importante de la varianza en los síntomas negativos, el rendimiento neurocognitivo y los resultados funcionales (Beck et al., 2018). Así mismo, existen distintos perfiles de déficit en cognición social según la presencia de síntomas positivos y negativos (Peyroux et al., 2019; Strassnig et al., 2018). No obstante, estos perfiles tan solo dan cuenta de una parte de las personas con síntomas psicóticos y sus resultados no pueden ser considerados definitivos.

Hay pocos estudios en sujetos de alto riesgo y los resultados obtenidos son poco concluyentes, si bien parece que la ToM es el dominio que más se relaciona con el funcionamiento social (Barbato et al., 2013; Cotter et al., 2017) aunque, en uno de los estudios revisados, los cortos periodos de seguimiento (apenas 6 meses) ponen en duda su valor predictivo. Sin embargo, los déficits en CS se 
siguen sugiriendo como un marcador de riesgo para padecer esquizofrenia, están presentes antes del inicio de la enfermedad y resultan más significativos que los déficits neurocognitivos (Barbato et al., 2015; Lee, Hong, Shin y Kwon, 2015).

Además, los sujetos que transitan desde un episodio psicótico hacia un diagnóstico de esquizofrenia muestran un mayor déficit en ToM que los que no lo hacen (Lee et al., 2015) y este déficit, influido a su vez por el razonamiento y la memoria verbal, ya se encuentra en los individuos con primeros episodios psicóticos (Catalan et al., 2018; Mallawaarachchi, Cotton, Anderson, Killackey y Allot, 2019).

Otros estudios muestran una consistente relación entre el procesamiento emocional y una amplia gama de actividades y conductas relacionadas con el desempeño sociolaboral y la vida independiente (Kee, Green, Mintz y Brekke, 2003). Por otro lado, la hostilidad, como estilo atributivo, también parece influir en la calidad de vida y correlaciona con altos niveles de síntomas positivos, ansiedad, depresión y malestar emocional general (Buck, Healey, Gagen, Roberts y Penn, 2016).

Por último, los déficits en CS se mantienen estables 12 meses después del primer brote psicótico, independientemente de la mejora de los síntomas, y son comparables a los que se constatan en las fases crónicas de la enfermedad (Green et al., 2012; Horan et al., 2012).

De esta manera, el déficit en CS no podría entenderse como el resultado de la cronicidad o de la gravedad de la enfermedad y algunos autores lo llegan a considerar como una característica prototípica de la esquizofrenia (Green et al., 2015). Además, parece que influye en la recuperación, incluyendo áreas como las relaciones interpersonales, el rendimiento educativo y los resultados vocacionales (Javed y Charles, 2018).

Sin embargo, la falta de consistencia de algunos de los hallazgos expuestos y el diseño transversal de muchos de ellos no permite establecer conclusiones firmes respecto a la capacidad predictiva de la CS sobre la recuperación a largo plazo. 
Por otro lado, cabe destacar el estudio longitudinal llevado a cabo durante 26 años por Jobe y Harrow (2010) donde observaron varios factores de riesgo relacionados con el estrés o con la vulnerabilidad a la psicosis y que explicarían el curso episódico que experimenta la mayoría de las personas con esquizofrenia. Estos factores interactúan con la personalidad, el temperamento y los sesgos cognitivos que, si bien no son los causantes de la psicosis, influyen en su evolución. La investigación se centró en dimensiones como la ansiedad-rasgo, un pobre desarrollo antes de la enfermedad, rasgos de personalidad como el locus de control, estilos cognitivos, el deterioro neurocognitivo, la duración de la psicosis no tratada o la evolución natural sin fármacos de algunos de los participantes (Harrow et al., 2015).

En conjunto, los estudios realizados sugieren que los déficits en CS no pueden explicarse únicamente por los déficits en funciones cognitivas básicas. Sin embargo, algunos autores (Fanning, Bell y Fiszdon, 2012) afirman que una "buena neurocognición" es necesaria, aunque no suficiente, para una adecuada CS y cuantifican que la neurocognición, sobre todo las habilidades en memoria y funciones ejecutivas, podría explicar hasta el 39\% de la varianza del rendimiento en pruebas de CS (Mehta, Bhagyavathi, Thirthalli, Kumar y Gangadhar, 2014).

Contrariamente a ello, algunos autores advierten cómo los déficits neurocognitivos en la esquizofrenia se han podido sobredimensionar, atendiendo a que, factores contextuales como la pobre motivación, la ansiedad de ejecución o la distracción debida a los propios síntomas durante las pruebas de evaluación, pudieran estar enmascarando los resultados, lo cual ha sido escasamente considerado en la investigación (Moritz et al., 2017) y representa una importante limitación a la hora de interpretar estos hallazgos.

Pocos trabajos investigan específicamente el impacto del JTC sobre la capacidad funcional en la esquizofrenia. Se ha estudiado la asociación de psicopatología, neuropsicología y JTC con la calidad de vida subjetiva, el rendimiento profesional y el ambiente familiar, encontrando que la sintomatología positiva es el mejor predictor del rendimiento profesional y los 
cambios a largo plazo (Andreou et al., 2014). Los déficits de memoria verbal se asociaron con el estado funcional actual, mientras que la capacidad cognitiva general predijo mejor los cambios funcionales a lo largo del tiempo. La mejora en el sesgo JTC afectó positivamente al rendimiento laboral (Andreou et al., 2014).

Además, diferentes perfiles metacognitivos se han relacionado con la presencia de sintomatología negativa (Popolo et al., 2017) y la metacognición, en general, con el funcionamiento social en la esquizofrenia crónica, independientemente de la psicopatología (James et al., 2016).

Por último, cabe destacar cómo la historia de abusos, los traumas infantiles y la sobreprotección parental se han relacionado con la metacognición, apareciendo el apego ansioso como predictor de las capacidades metacognitivas (Aydin et al., 2016). Los patrones de apego y las adversidades durante la infancia son relevantes para entender el papel de los factores psicosociales y ambientales en la vulnerabilidad y el desarrollo de la psicosis (Bailey et al., 2018; Sheinbaum, Bedoya, Ros-Morente, Kwapil y Barrantes-Vidal, 2013). Las medidas de apego se correlacionan con fenómenos psicóticos, incluida la paranoia (Wickham y Bentall, 2016) y la angustia al escuchar voces (Berry, Wearden, Barrowclough, Oakland y Bradley, 2012). Además, el estilo de apego se asocia con los sesgos atribucionales (Donohoe et al., 2008) y la mentalización (MacBeth, Gumley, Schwannauer y Fisher, 2011); con problemas interpersonales y de funcionamiento social (Berry, Barrowclough y Wearden, 2008); con la CS como mediador entre el apego inseguro y los problemas clínicos y de funcionamiento social (Korver-Nieberg, Berry, Meijer y De Haan, 2014), y con experiencias psicóticas en población subclínica (Gawęda et al., 2018). Algunas investigaciones también han sugerido que el tipo de apego puede estar relacionado con la forma en que las personas con psicosis se involucran con los servicios de salud mental (McGonagle, Bucci, Varese, Raphael y Berry, 2019; White, Haddock y Varese, 2019). 


\subsection{Programas de intervención}

\subsubsection{Intervenciones sobre la cognición}

Numerosas intervenciones han sido diseñadas específicamente para mejorar la cognición, tales como la Terapia Psicológica Integrada (Brenner, Hodel, Roder y Corrigan, 1992), la Terapia Neurocognitiva Integrada (Müller y Roder, 2010), la Terapia de Rehabilitación Cognitiva (Penadés et al., 2012) y la Cognitive Enhancement Therapy (Hogarty y Flesher, 1999). Estas intervenciones han mostrado un éxito limitado en la esquizofrenia, pero animan al desarrollo de terapias más específicas (Brown, Tas y Brüne, 2012). Por ejemplo, el Programa de Rehabilitación Cognitiva en Psicosis (REHACOP) aborda áreas como la atención, lenguaje, aprendizaje y memoria, actividades de la vida diaria, funciones ejecutivas, habilidades sociales, cognición social y psicoeducación (Ojeda et al., 2012). Los resultados sugieren que puede mejorar el rendimiento neuropsicológico independientemente del grado de deterioro y del estado evolutivo de la enfermedad (Ojeda et al., 2012). En líneas generales, los programas de rehabilitación cognitiva se muestran más efectivos cuando se integran con programas de rehabilitación psicosocial, ya que permiten que los individuos practiquen las habilidades cognitivas adquiridas en entornos del mundo real (Brown et al., 2012).

A continuación se detallan algunos de los programas que muestran un especial interés en el tratamiento de la cognición social en las psicosis:

A) El programa de Entrenamiento en Interacción y CS (SCIT, del inglés: Social Cognition and Interaction Training) es una intervención grupal manualizada dirigida a entrenar la percepción emocional, la ToM y el sesgo atribucional (Penn, Roberts, Combs y Sterne, 2007). Los resultados de un estudio de seguimiento mostraron que los efectos sobre el funcionamiento social se mantuvieron durante 6 meses (Combs et al., 2009). 
B) El Entrenamiento en cognición social y Metacognición (MSCT, del inglés: Metacognitive and Social Cognition Training) se diseñó para "eliminar los déficits y corregir los sesgos en CS" y ha demostrado mejorar la ToM, percepción social, reconocimiento de emociones y funcionamiento social, además de reducir significativamente la tendencia a saltar a conclusiones (Rocha y Queirós, 2013).

C) La Metacognitive Reflection and Insight Therapy (MERIT) se diseñó para mejorar los dominios de la metacognición. Los resultados de un estudio indicaron que las personas que siguieron el programa mejoraron en autorreflexión y control metacognitivo, pero no en medidas de síntomas, calidad de vida y funcionamiento social (De Jong et al., 2019).

D) El Programa de Entrenamiento en Cognición Social (PECS), desarrollado en España dentro del Proyecto SCORES (Social Cognition on Rehabilitation in Schizophrenia) se diseñó para entrenar de manera específica la CS en pacientes con esquizofrenia. El PECS está disponible de manera gratuita en la página web del proyecto (http://www.proyectoscores.es/pecs.php). Se mostró útil en la mejora de la ToM, así como en el reconocimiento de emociones en pacientes ambulatorios con esquizofrenia (Gil-Sanz, FernándezModamio, Bengochea-Seco, Arrieta-Rodríguez y Pérez-Fuentes, 2016). Para medir la capacidad de reconocimiento de emociones, se diseñó y validó una nueva herramienta de aplicación en la esquizofrenia. La Prueba de Evaluación del Reconocimiento de Emociones (PERE) está formada por 56 fotografías que valoran la percepción de las 6 emociones básicas: alegría, tristeza, enfado, sorpresa, miedo y asco (Gil-Sanz et al., 2017).

E) El e-Motional Training $®$ es un nuevo programa de entrenamiento online en CS, aplicado y supervisado por un clínico. Incluye varios módulos de entrenamiento en percepción emocional y un corto de animación para entrenar la ToM y el estilo atribucional. Los resultados preliminares muestran la viabilidad de la intervención y su posible eficacia en la mejora del 
reconocimiento emocional, ToM y estilo atribucional (Vázquez-Campo et al., 2016).

Los resultados de un metaanálisis sobre la eficacia de los programas de entrenamiento en CS mostraron apoyo preliminar para su uso en la esquizofrenia (Kurtz et al., 2016) y reconocen la CS como un componente básico en el proceso de rehabilitación (Horan y Green, 2019). La evidencia actual, aunque limitada, indica que la mayoría de los programas desarrollados hasta la fecha mejoran los dominios de la CS para los que fueron diseñados (Mehta et al., 2014). Sin embargo, algún estudio encuentra que la mejoría se produjo en habilidades distintas a las pretendidas (Vaskinn et al., 2019), lo que resta validez a los hallazgos descritos. Como se describió en el capítulo anterior, recientes hallazgos demuestran que una cuarta parte de los pacientes no presentan deterioro en la CS. La existencia de distintos grados de deterioro indica que los esfuerzos de rehabilitación pueden no ser necesarios para todos los individuos (Hajdúk et al., 2018). En este sentido, se recomienda mayor investigación acerca de las dianas terapéuticas y los componentes específicos de cada programa (Wykes, 2018).

\subsubsection{Intervención sobre sesgos cognitivos}

El entrenamiento metacognitivo para la esquizofrenia (MCT) es una intervención basada en conceptos de la psicoeducación, la rehabilitación cognitiva, el entrenamiento en CS, la ToM y la terapia cognitivo-conductual. El programa tiene como objetivo modificar errores y sesgos cognitivos comunes en la esquizofrenia, partiendo del supuesto de que estas distorsiones pueden evolucionar hasta la creación de falsas creencias o, en última instancia, convertirse en delirios (Monfort-Escrig y Pena-Garijo, 2016). Se desarrolla a través de ocho módulos y dos módulos adicionales (Tabla 7). Tiene por objeto aumentar la conciencia sobre cada una de las distorsiones y enseñar a reflexionar sobre ellas de una forma crítica, para cambiar y ampliar el actual repertorio de habilidades cognitivas (Monfort-Escrig y Pena-Garijo, 2016). 
Varios estudios han demostrado una reducción moderada en la gravedad de los delirios y una mejoría en la atribución causal, en el conocimiento de la enfermedad y en la reducción de los síntomas depresivos (Favrod et al., 2014). Dos recientes metaanálisis mostraron que el MCT ejercía un efecto entre pequeño y moderado en los síntomas positivos, y una muy buena aceptabilidad (Eichner y Berna, 2016). Además, la modalidad individual (MCT+) mostró un mayor efecto beneficioso para ser aplicado a pacientes con esquizofrenia o trastorno delirante, por lo que los autores recomiendan su uso (Liu et al., 2018).

\section{Tabla 7. Módulos del Entrenamiento Metacognitivo (MCT)}

\begin{tabular}{ll}
\hline Sesiones & Sesgo cognitivo \\
\hline Módulo 1 & Atribuciones \\
Módulos 2 y 7 & Salto a conclusiones \\
Módulo 3 & BADE \\
Módulos 4 y 6 & Empatizar \\
Módulo 5 & Exceso de confianza en los errores de memoria \\
Módulo 8 & Patrones depresivos \\
Módulo adicional I & Autoestima \\
Módulo adicional II & Estigma \\
\hline
\end{tabular}

\subsubsection{Correlatos neurales de las intervenciones sobre cognición}

Una revisión sistemática sobre los correlatos neurales de la rehabilitación cognitiva en la esquizofrenia proporciona observaciones prometedoras sobre su posible efecto de neuroplasticidad (Isaac y Januel, 2016). Los resultados mostraron que, tras la terapia, se producía un aumento de la actividad en regiones prefrontales, cíngulo anterior y regiones occipitales durante el desempeño de tareas ejecutivas y de memoria de trabajo. Varios estudios encuentran una conectividad funcional mejorada después del entrenamiento, lo que sugiere dicho efecto neuroplástico a través de mecanismos de reorganización funcional. En este sentido, la suma del entrenamiento en CS y en metacognición 
podrían tener un efecto acumulativo sobre las redes neuronales involucradas en la CS (Isaac y Januel, 2016). También es interesante señalar cómo la integración funcional de las redes cognitivas de orden superior aparece intacta en la psicosis temprana, pero muestra signos de envejecimiento acelerado, lo que advierte del valor potencial de intervenciones dirigidas a la cognición durante las fases iniciales de la enfermedad (Sheffield et al., 2019). 
MARCO EXPERIMENTAL 


\section{Objetivos e hipótesis de la investigación}

\section{Descripción de los objetivos e hipótesis}

El marco experimental se compone de cuatro estudios que intentan dar cuenta de las relaciones entre los diferentes componentes desarrollados en el marco teórico, que parecen influir en la sintomatología y el funcionamiento social de las personas con trastornos del espectro de la esquizofrenia.

- El objetivo general del presente trabajo es explorar las asociaciones entre los constructos descritos en el marco teórico (las dimensiones del apego, la neurocognición y los dominios de la cognición social) con las variables clínicas y de funcionamiento social en una muestra de personas con trastornos psicóticos.

- Como objetivos específicos, pretendemos estudiar la influencia de los estilos atribucionales (estudio 1) y las diferentes dimensiones del apego (estudio 2) sobre distintas áreas del funcionamiento social. Por otro lado, desde una perspectiva dimensional en el estudio de la psicopatología, nos proponemos investigar el papel más o menos central que los aspectos incluidos en el objetivo general desempeñan dentro de una red interactiva formada por las dimensions del apego, la cognición y los síntomas psicóticos (estudio 3). Finalmente, intentaremos resumir cómo todas las variables de interés evaluadas en los anteriores estudios diferencian a la población clínica de la población normal y en qué sentido influyen en el funcionamiento social de los individuos con trastornos psicóticos (estudio 4).

A continuación se detallan los objetivos generales y específicos, así como las hipótesis generadas para cada estudio en concreto. 


\section{Estudio 1: Los estilos atribucionales y el funcionamiento social en la}

esquizofrenia. ¿Es adecuado el Modelo de la Indefensión Aprendida?

El objetivo general del estudio es confirmar la adecuación del Modelo de Indefensión Aprendida para predecir la sintomatología depresiva en personas con trastornos del espectro de la esquizofrenia, y su posible utilidad para predecir los síntomas psicóticos y el funcionamiento social.

Objetivos específicos:

1. Comparar los estilos atribucionales entre un grupo de personas con diagnóstico de trastornos del espectro de la esquizofrenia y un grupo no clínico.

2. Estudiar las posibles relaciones entre los estilos atribucionales y la severidad de síntomas de depresión, las creencias delirantes y otros síntomas psicóticos en el grupo clínico.

3. Generar modelos predictivos de funcionamiento social a través de los estilos de atribución.

Hipótesis:

1. Se encontrarán diferencias significativas en los estilos atribucionales entre los grupos, siendo éste más negativo en el grupo clínico.

2. El grupo clínico mostrará un "patrón depresivo":

2.1. Una atribución interna, estable y global para las situaciones negativas,

2.2. Un patrón de atribución externo, inestable y específico para las situaciones positivas,

2.3. Un estilo "pesimista" mayor.

3. Los resultados replicarán los encontrados en los estudios clásicos (Lysaker et al. 2004) que observaron que un sesgo de atribución inestable se asocia con un peor funcionamiento social en la psicosis, independientemente de los síntomas. 


\section{Estudio 2: Las dimensiones del apego como predictores del funcionamiento}

social en la esquizofrenia

El objetivo principal del estudio es comprobar si una medida dimensional como el CAMIR-R puede diferenciar los estilos de apego comparando un grupo de individuos con trastornos del espectro de la esquizofrenia con una muestra no clínica, y si, además, estas dimensiones de apego pueden predecir el funcionamiento social dentro del grupo clínico.

Objetivos específicos:

1. Dada la ausencia de estudios previos con población psicótica, el primer objetivo es probar que el instrumento para la evaluación del apego adulto, CAMIR-R, es adecuado para evaluar las dimensiones de apego en personas con trastornos del espectro de la esquizofrenia.

2. Diferenciar perfiles de apego específicos entre ambos grupos.

3. Observar las relaciones de los síntomas y las dimensiones de apego sobre el funcionamiento social en el grupo clínico.

Hipótesis:

1. El CAMIR-R resultará ser un instrumento adecuado para evaluar las dimensiones de apego en personas con psicosis.

2. El CAMIR-R diferenciará perfiles de apego entre el grupo clínico y el grupo no clínico, observándose mayor apego inseguro en el grupo clínico.

3. Se encontrará que:

3.1. Los síntomas y las dimensiones de apego predecirán áreas del funcionamiento social en el grupo clínico.

3.2. Las dimensiones de apego estarán directamente relacionadas con el funcionamiento social, independientemente de la severidad de los síntomas. 
Estudio 3: La centralidad del apego seguro en una red interactiva entre los síntomas psicóticos, la cognición y las dimensiones del apego

El objetivo general del estudio es explorar la interacción entre un conjunto de variables relacionadas con el apego, los dominios de la cognición y los síntomas psicóticos en una muestra de personas con trastornos del espectro de la esquizofrenia.

Objetivos:

1. Generar un modelo de red interactiva entre las variables de interés.

2. Explorar qué variables juegan un papel más o menos central dentro de la red (nodos).

3. Testar la fiabilidad de nuestro modelo, utilizando algunos nuevos métodos recomendados para estimar las redes psicológicas y su precisión.

Hipótesis:

1. Aunque el estudio es parcialmente exploratorio, teniendo en cuenta las teorías que vinculan el apego con la cognición social y los síntomas psicóticos, proponemos que el apego seguro/inseguro ocupará un lugar central dentro de la red interactiva de síntomas, dominios cognitivos y dimensiones del apego.

2. El apego seguro se relacionará con los síntomas y mediará parcialmente la relación con los demás nodos.

\section{Estudio 4: Predictores del funcionamiento social en personas con} trastornos del espectro de la esquizofrenia

El principal objetivo de este estudio es el de explorar cómo la neurocognición, la cognición social, las dimensiones de apego y el funcionamiento social se ven afectados a través de varias condiciones clínicas. 
Objetivos específicos:

1. Explorar cómo las variables de funcionamiento social, neurocognición, cognición social y dimensiones de apego están afectadas en los grupos clínicos en comparación con la muestra de personas no clínicas.

2. Observar las diferencias entre las tres condiciones de estudio, atendiendo a la gravedad de los déficits.

3. Investigar cómo los síntomas, los dominios de la cognición y las dimensiones de apego influyen en el funcionamiento social.

\section{Hipótesis:}

1. Las personas con trastornos del espectro de la esquizofrenia mostrarán significativas deficiencias en el funcionamiento social, la neurocognición, la cognición social y las dimensiones de apego en comparación con el grupo de personas no clínicas.

2. Se observará una "gradación" de los déficits a través de las tres condiciones estudiadas.

3. La influencia del apego y la cognición en el funcionamiento social estará parcialmente mediada por la gravedad de los síntomas.

\section{Metodología}

\section{Participantes y procedimiento}

Las características clínicas y demográficas de los sujetos participantes se describen en cada uno de los estudios.

Los pacientes del grupo clínico con trastornos del espectro de la esquizofrenia según los criterios del DSM-5 (APA, 2013) fueron reclutados en dispositivos de salud mental de Castellón y Valencia. Los criterios de inclusión fueron: 1) diagnóstico de esquizofrenia, trastorno esquizoafectivo u otro trastorno psicótico; 2) edad entre 18-65 años; 3) estabilidad en los criterios 
clínicos; 4) estabilidad en la medicación; 5) sin historia de drogas en las últimas cuatro semanas; 6) habilidad para proveer el consentimiento informado por escrito; 7) completar la batería de evaluación; y 8) poseer un nivel de español nativo.

El grupo no clínico incluyó a participantes sin historia de problemas de salud mental, reclutados como muestra de conveniencia en la misma área geográfica.

El procedimiento que se llevó a cabo consistió en diversas fases: 1) desarrollo y definición de los criterios de inclusión de las personas participantes; 2) búsqueda de la muestra clínica en unidades de salud mental y dispositivos de atención comunitaria; 3) toma de contacto, explicación de la investigación y consentimiento informado de las personas participantes; 4) pase de los instrumentos de evaluación; y 5) creación de la matriz de datos codificada.

La evaluación se realizó en una única sesión de 120 minutos o en dos sesiones dependiendo de la persona participante en concreto. Su aplicación se llevó a cabo de manera individual o, cuando fue posible, en grupos de máximo cuatro personas. Algunos instrumentos permitían la administración de un dossier de cuestionarios autoaplicables, como el CAMIR-R o el ASQ, y otras pruebas como la PERE y la Beads Task podían aplicarse colectivamente con la ayuda de un soporte visual. La evaluación clínica en la mayoría de los casos se había llevado a cabo previamente por el/la profesional, actualizando los datos a la situación actual.

\section{Instrumentos de evaluación}

\section{Evaluación clínica:}

- Escala del síndrome positivo y negativo (PANSS; Kay et al., 1987; Peralta y Cuesta, 1994). Se trata de una entrevista semi-estructurada de 30 ítems que evalúan el síndrome esquizofrénico desde la doble perspectiva dimensional y categorial. Se obtiene puntuación para los síntomas positivos, síntomas negativos y psicopatología general. Además se puede obtener una puntuación 
global que sirve como medida de la gravedad de los síntomas psiquiátricos. El coeficiente alfa de Cronbach es de .73 para la escala positiva, .83 para la escala negativa y .87 para la escala de psicopatología general.

- Adaptación española del inventario de ideas delirantes de Peters (PDI-21; Peters, Joseph, Day y Garety, 2004; López-Ilundain, Pérez-Nievas, Otero y Mata, 2006). Es un instrumento heteroaplicado que consta de un total de 21 ítems. Se obtiene una puntuación global y tres subescalas que miden el grado de convicción, preocupación y estrés. Este instrumento ha mostrado una consistencia interna con coeficiente alfa de .91.

- Inventario de depresión de Beck II (BDI-II; Beck, Ward, Mendelson, Mock y Erbaugh, 1961; Sanz, Perdigón y Vázquez, 2003). Es una escala autoaplicada de 21 ítems que proporciona mediante una puntuación total, una medida de la presencia de depresión y su gravedad. El BDI-II ha obtenido coeficientes alfa para población española de .88 en estudiantes universitarios, .88 en población general, y .92 en pacientes.

\section{Evaluación del funcionamiento social:}

- Versión reducida de la escala de funcionamiento social (SFS-R; Alonso et al., 2008). La escala original (SFS; Torres y Olivares, 2005) fue diseñada para la evaluación del funcionamiento social en personas con esquizofrenia. La SFS reducida proporciona una puntuación total y también incluye puntuaciones para cinco áreas: 1) aislamiento y actividades sociales, 2) comunicación interpersonal, 3) independencia-ejecución, 4) independencia-competencia, y 5) empleo-ocupación. La escala se ha mostrado válida y fiable para la práctica clínica con un coeficiente alfa de Cronbach de .76. En esta investigación se ha prescindido de la escala de empleo-ocupación. La puntuación de empleoocupación se ha reemplazado por el estado laboral actual. 


\section{Evaluación de la cognición social:}

- Test de las Insinuaciones, adaptación española de la prueba Hinting Task (Corcoran, Mercer y Frith, 1995; Gil, Fernández-Modamio, Bengochea y Arrieta, 2012). La prueba, compuesta por diez historias breves, ha sido diseñada de manera específica para la evaluación de la teoría de la mente en personas con diagnóstico de esquizofrenia. Se puede obtener una puntuación global para las diez historias y una puntuación global para cinco historias. La versión reducida de cinco historias ha obtenido mejor consistencia interna con coeficiente alfa de Cronbach de .73 para sujetos control y .78 para participantes.

\section{Evaluación del estilo atribucional:}

- Cuestionario del Estilo Atribucional, adaptación española (ASQ; Peterson, Semmel, Seligman et al., 1982; Sanjuán y Magallares, 2005, 2006). Consiste en 12 situaciones hipotéticas, 6 positivas y 6 negativas. Se puede obtener dos puntuaciones globales para situaciones positivas y para situaciones negativas; tres puntuaciones para ambas situaciones (interna, externa y global); y una puntuación compuesta (la diferencia entre las puntuaciones globales positiva y negativa) para obtener una medida del estilo de explicación del sujeto en términos de pesimismo u optimismo. De acuerdo con Sanjuán et al. (2013) la versión original obtuvo una consistencia interna de coeficiente alfa entre .70 y .73 para el estilo de atribución negativo, obteniendo mejores propiedades la versión española (alfa de Cronbach entre .74 y .80).

\section{Evaluación del sesgo de razonamiento saltar a conclusiones:}

- Prueba Beads Task (Dudley y Over, 2003). Se trata de una tarea de razonamiento probabilístico en que se muestra al sujeto una imagen de dos tarros llenos de bolas de dos colores distintos en distinta proporción. En el presente estudio se utilizó una versión computarizada con proporción de bolas 60:40. Se informa a los participantes que aleatoriamente se ha 
seleccionado uno de los frascos y se extraerán bolas de él que serán presentadas una a una en la pantalla. La tarea del sujeto consiste en decidir de qué frasco provienen las bolas, pudiendo ver tantas bolas como quiera hasta estar completamente seguro de su decisión. Se considera la existencia del sesgo de salto a conclusiones cuando la persona emite su juicio después de extraer 1 o 2 bolas (Garety et al., 2011).

\section{Evaluación del reconocimiento de emociones:}

- Prueba de Evaluación de Reconocimiento de Emociones (PERE; Gil-Sanz et al., 2017). Se trata de una test formado por 56 fotografías ( 28 de hombres y 28 de mujeres), que valora la percepción de seis emociones básicas: alegría, tristeza, enfado, sorpresa, miedo y asco, además de incluir una expresión neutra. Las fotografía obtuvieron una precisión superior al 89 \% y una fiabilidad testretest entre 0.80 y 1.32 para los controles y entre 0.61 y 1.92 para los pacientes ambulatorios.

\section{Evaluación del apego:}

- Versión corta del cuestionario de apego CAMIR-R (Pierrehumbert et al., 1996; Balluerka, Lacasa, Gorostiaga, Muela y Pierrehumbert, 2011). Este cuestionario mide las representaciones de apego y la concepción del funcionamiento familiar en la adolescencia y principio de la edad adulta. Consta de 32 ítems y evalúa 7 dimensiones: 1) Seguridad: Disponibilidad y apoyo de las figuras de apego; 2) Preocupación familiar; 3) Interferencia de los padres; 4) Valor de la autoridad de los padres; 5) Permisividad parental; 6) Autosuficiencia y rencor contra los padres; y 7) Traumatismo infantil. Estas dimensiones se relacionan con tres estilos de apego: seguro, preocupado y evitativo. Los índices de consistencia interna se sitúan entre .60 y .85 . 


\section{Evaluación de la neurocognición:}

- Screening del Deterioro Cognitivo en Psiquiatría (SCIP-S; Pino, Guilera, Rojo, Gómez-Benito y Purdon, 2014). Se trata de una prueba breve de cribado del deterioro cognitivo especialmente diseñada para la detección de niveles bajos de rendimiento cognitivo en pacientes psiquiátricos diagnosticados de esquizofrenia y otras psicosis, y trastorno bipolar, delirante. La prueba ofrece una puntuación general de la ejecución global y puntuaciones para 5 factores: aprendizaje verbal inmediato y diferido, memoria de trabajo, fluidez verbal y velocidad de procesamiento. La consistencia interna se sitúa en .74. Para el propósito de la investigación solo se ha incluido en el análisis la puntuación global.

\section{Análisis de datos}

Los análisis estadísticos se llevaron a cabo mediante los paquetes estadísticos SPSS (versiones 23.0 y 25.0) y el programa JASP (versión 0.13.1) que incluye el paquete R para análisis de redes y ecuaciones estructurales.

Los estadísticos descriptivos y las comparaciones entre grupos se calcularon con pruebas paramétricas o no paramétricas según lo requirieran las características de distribución de los datos en cada estudio. Usamos pruebas $t$ para comparaciones entre grupos con distribución normal y la $d$ de Cohen para calcular el tamaño del efecto. Se utilizaron ANOVAs para comparaciones múltiples. La prueba de Kruskal-Wallis se utilizó para comprobar las diferencias significativas entre los grupos. Cuando no se cumplió la presunción de homogeneidad, se utilizó la corrección de Welch, y se seleccionó la $\eta^{2}$ parcial (etacuadrada parcial) como una estimación del tamaño del efecto. Para las comparaciones posthoc, se aplicó la corrección de Dunn.

Se usaron correlaciones bivariadas y análisis de regresión para estudiar las relaciones entre las variables estudiadas. 
Para el análisis de red se utilizó el método de correlaciones parciales. Para minimizar las posibles correlaciones espúreas, se utilizó una técnica llamada LASSO: "least absolute shrinkage and selection operator".

Para probar si existían efectos indirectos en el análisis de mediación, utilizamos el método bootstrap (1000 replicaciones). El análisis se llevó a cabo mediante modelado de ecuaciones estructurales (SEM) utilizando la sintaxis lavaan (Rosseel, 2012) con el software proporcionado por el paquete JASP.

Para todos los casos se consideró un umbral mínimo de significación estadística de $p<0.05$.

La explicación detallada de cada método se describe en el estudio correspondiente.

\section{Otras consideraciones}

Los estudios se detallan a modo de artículos de investigación; ya que, o bien han sido publicados, o están en procesos de revisión para su posible publicación. Por este motivo, se mantiene el idioma y el formato de la revista científica en cuestión, aunque se ha editado el diseño de los mismos para la correcta adecuación con este trabajo. No obstante, para facilitar la comprensión de los mismos, aquí hemos aportado los aspectos más importantes en cuanto a los objetivos y la metodología. Por otro lado, al principio de cada estudio se facilita un resumen en castellano. Las consideraciones referentes a los resultados más relevantes se volverán a resumir al final de este marco experimental en el apartado de discusión general. 


\section{Capítulo 5 Estudio 1}

\section{Los estilos atribucionales y el funcionamiento social en la esquizofrenia. ¿Es adecuado el modelo de la indefensión aprendida? ${ }^{3}$}

\section{Resumen}

El estudio analizó la adecuación del modelo de Indefensión Aprendida y la Teoría de la Desesperanza para predecir síntomas y funcionamiento social en personas con trastornos psicóticos comparando un grupo clínico $(n=25)$ con una muestra no-clínica $(n=30)$. Se calcularon correlaciones bivariadas y regresiones lineales para evaluar relaciones entre el cuestionario de Estilos Atribucionales (ASQ), variables clínicas y funcionamiento social dentro del grupo clínico. Se encontraron diferencias para la mayoría de las dimensiones atribucionales. Un "estilo pesimista" predijo la depresión dentro del grupo clínico. Un sesgo de atribución inestable predijo los síntomas positivos. La psicopatología y un estilo de atribución global predijeron un $50 \%$ de la varianza del funcionamiento social. El modelo de Indefensión Aprendida resultó adecuado para predecir la depresión independientemente de los síntomas psicóticos y fue útil prediciendo los síntomas clínicos y el funcionamiento social en personas con trastornos del espectro de la esquizofrenia. Se discuten las implicaciones teóricas y clínicas.

\footnotetext{
${ }^{3} \mathrm{El}$ presente estudio ha sido publicado como artículo empírico en:

Monfort-Escrig, C., \& Pena-Garijo, J. (2020). Attributional styles and social functioning in schizophrenia. Is the learned helplessness model suitable? Clínica y Salud. Ahead of print. https://doi.org/10.5093/ clysa2020a21
} 


\title{
Attributional Styles and Social Functioning in Schizophrenia. Is the Learned Helplessness Model Suitable?
}

\author{
Cristina Monfort-Escrig and Josep Pena-Garijo \\ Jaume I University, Castellón de la Plana, Spain
}

\begin{abstract}
We tried to verify whether the reformulated "Learned Helplessness Model" and its more fully articulated form, the "Hopelessness Theory of Depression", were adequate to predict symptoms and Social Functioning (SF) in persons with schizophrenia-spectrum disorders by comparing a clinical group $(N=25)$ with a non-clinical one $(N=30)$. Bivariate correlations and multiple linear regressions were conducted to assess relationships between the Attribution Styles Questionnaire (ASQ), clinical variables and SF within the clinical group. Differences between groups were found for most of attributional dimensions. A "pessimistic style" predicted depression within the clinical group. An unstable attribution bias predicted positive symptoms. General psychopathology and a global attribution style predicted $50 \%$ of the variance in general SF. The Learned Helplessness Model was suitable to predict depression regardless of psychotic symptoms and may be useful to predict clinical symptoms and SF in persons with schizophrenia spectrum disorders. Clinical and theoretical implications were discussed.
\end{abstract}

Keywords: Attributional style. ASQ. Learned helplessness. Schizophrenia. Social functioning. 


\section{Introduction}

Attributions and related biases are particularly relevant to the development and maintenance of positive symptoms in psychosis (Howes \& Murray, 2014) and depressive symptoms in mood and psychotic disorders (Davidson et al., 2018). Some studies in psychosis show that the attributional style is probably a separate construct of social cognition since it minimally correlates with other domains (Buck et al., 2016). Bentall et al. (2001) differentiate two biases: a general tendency to make non-self-attributions, including people and circumstances, for positive events ("externalising bias"); and a specific tendency to blame others for negative events ("personalising bias"). Individuals who usually tend to explain disturbing events in terms of external (non-self) causes may be particularly prone to develop delusional beliefs about such experiences (Langdon et al., 2013). On the other hand, several studies observed a higher tendency to attribute positive events (but not negative) to oneself (So et al., 2015), but other studies did not (Achim et al., 2016). Moreover, persecutory delusions caused by an externalising attributional bias maintain selfesteem preserved in persons with delusions (Murphy et al., 2018). By contrast, later research from the "defence model" of persecutory delusions predicts that people with this kind of delusions have lower self-esteem than people with depression and a greater externalising bias (Murphy et al., 2018). Connecting depression and psychosis, the Learned Helplessness Model (Abramson et al., 1978) and the Hopelessness Theory of Depression (Liu et al., 2015), provided an important framework for explaining the development of depressive symptoms through specific attributional patterns and had an initial use in the field of psychosis. These initial investigations showed that persons with depression present an abolished "self-serving bias", often named "depressive realism", or a tendency to internalize blame for negative events (Moritz et al., 2007) and found the opposite pattern in persons with paranoid delusions, including an excessive tendency to attribute positive events to oneself and negative events to external causes, indicating an increased "self-serving bias" (Moritz et al., 2018). However, 
the way these biases influenced social functioning (SF) remained unexplored. Lysaker et al. (2004) using the Attributional Style Questionnaire (ASQ; Peterson et al., 1982), the main instrument derived from the attributional theory of depression, revealed inconsistencies of the model when relating attributive style to positive psychotic symptoms (externalising bias) and found that it is the tendency to see life events as the result of unstable or unpredictable causes (unstable attribution bias) which is associated with a worse SF in psychosis, regardless of symptoms. However, recent studies notice that several attributional biases as hostility, guilt and aggressiveness are not associated with SF, unlike other social cognition domains, such as Theory of Mind or emotion perception (Saffarian et al., 2018).

Currently, the prevalence of depressive disorders in schizophrenia is estimated at around $40 \%$ and is correlated with poorer outcomes (Berardelli et al., 2019). Beyond this, "hopelessness", regardless of depressive symptoms, predicts a worse global functioning and is an important risk factor for suicidal behaviour (Cassidy et al., 2018). Given the importance of depressive symptoms (Berardelli et al., 2019; Davidson et al., 2018) and the increased "helplessness" (a bias to externalise both positive and negative events) found in persons with psychosis (Lincoln et al., 2010), the present study aimed to verify whether the reformulated Learned Helplessness Model and the more fully articulated form, Hopelessness Theory of Depression, may be specifically suitable to predict depressive symptoms in persons with schizophrenia-spectrum disorders. Moreover, our principal focus is not to demonstrate the relationship between attribution styles and psychotic symptoms that influent models satisfactorily explain (Bentall et al., 2001; Garety \& Freeman, 2013; Moritz et al., 2018; Murphy et al., 2018) but to test their utility to explain increased depressive patterns and predict SF in persons with schizophrenia-spectrum disorders. A cross-sectional study was designed with the following objectives: 1) to compare attributional styles between a group of persons diagnosed with schizophrenia-spectrum disorders versus a non-clinical group; 2) to check possible relationships between attributional styles and the severity of depression, delusional beliefs and other 
psychotic symptoms within the clinical group; and 3) to generate prediction models of SF through attributional dimensions.

We hypothesize that: 1) there will be significant differences in attribution styles between groups; 2) the clinical group will show an increased "depressive pattern" (internal, stable and global attribution for negative situations, and the opposite pattern for positive ones) and a more "pessimistic" style; 3) an internal, stable and global attribution for negative events (and the opposite pattern for positive ones) will predict depressive symptoms within the clinical group; 4) results will replicate classical studies in the field (Lysaker et al., 2004), finding that an unstable attribution bias is associated with a worse SF in psychosis, regardless of symptoms. We expect to confirm the suitability of the Learned Helplessness Model to predict depression and a "hopelessness pattern" in persons with schizophrenia-spectrum disorders, and its possible utility in predicting psychotic symptoms and SF.

\section{Method}

\section{Participants and Procedure}

A total of 55 adults participated voluntarily in the study. The initial clinical group involved thirty individuals diagnosed with a psychotic disorder according to Diagnostic and Statistical Manual of Mental Disorders, 5th edition (American Psychiatric Association, 2013). Five patients didn't complete the full evaluation and were removed from the study. Finally, 25 participants composed the clinical sample. They were recruited via public mental health centres from Castellon and Valencia (Spain). The inclusion criteria were: 1) diagnosis of schizophrenia, schizoaffective or other psychotic disorder; 2) aged 18-65; 3) clinical stability; 4) medication-stabilised condition; 5) no history of drug abuse in the last four weeks; 6) ability to provide the written informed consent; 7) native Spanish language; and 8) complete all assessment measures. Seventeen participants (68\%) had a diagnosis of Schizophrenia. 6 (24\%) were outpatients; 9 (36\%) 
were attended in a day rehabilitation centre, and 10 (40\%) were inpatients. The non-clinical group involved 30 individuals with no history of mental health problems, recruited as a convenience sample (age and gender) in the same geographic area. Demographic data were collected and the Attribution Style Questionnaire (ASQ) was completed. No measure of psychiatric symptoms was obtained, except the general question: "Have you ever had mental health problems?". Assessment procedure was conducted by the first author (a Psychologist with a master's degree and $\mathrm{PhD}$ student) who was trained and supervised by the second author (a senior Clinical Psychologist with a PhD degree). The study complied with the Declaration of Helsinki ethical standards. The aim and conditions were verbal and written described to prospective participants and all of them provided written informed consent.

\section{Instruments}

Attributional styles were assessed using the Spanish version of the Attributional Style Questionnaire (ASQ; Peterson et al., 1982; Sanjuán et al., 2009). It consists of 12 hypothetical situations, 6 positive and 6 negative. A score can be obtained for each of the attributional dimensions (internal, stable and global) for both positive and negative events separately; two global scores, corresponding to the attributional styles for positive and negative situations; three dimensions for internal, stable or global bias for all events, a self-serving bias, a score that would reflect the tendency to over-attribute positive events to internal causes and negative events to external causes, and a composite index that fits the subject's explanatory style in terms of pessimism or optimism. According to Sanjuán et al. (2013), original version obtained internal consistency of alpha coefficients between .70 and .73 for the negative attributional style, with better properties for the Spanish version (Cronbach' alpha between .74 and .80).

Symptom severity was evaluates using the Positive and Negative Syndrome Scale (PANSS) (Kay et al., 1987; Peralta \& Cuesta, 1994). It is a semi-structured interview comprising 30 items, which assess positive and negative symptoms and 
general psychopathology. Summing subscales scores can derive a total symptom score. The instrument was shown to be reliable with Cronbach' alpha coefficients of .73 for the Positive Syndrome scale, .83 for the Negative Syndrome and .79 for the General Psychopathology scale (Kay et al., 1987).

Peters Delusions Inventory (PDI-21; Peters et al., 2004; López-Ilundain et al., 2006) is a hetero-applied instrument consisting of a total of 21 items. A total score and three subscales (conviction, worry and stress) can be obtained. The inventory has shown an internal consistency coefficient of .91.

The Beck Depression Inventory-II (BDI-II; Beck et al., 1996; Sanz et al., 2003 ) is a self-applied 21-item scale. Its objective is to identify and measure the severity of the typical symptoms of depression in adults and adolescents. The scale provides a total score that is the sum of the scores on each of the items. BDIII obtained alpha coefficients of .88 for general population and .92 for patients.

Social functioning was assessed using the Spanish short version of the Social Functioning Scale (SFS-R; Alonso et al., 2008). SFS (Torres \& Olivares, 2005 ) was designed to evaluate SF in schizophrenia through a medical interview. The original' 78 items were reduced to 15 . The SFS-R includes five areas: 1) social isolation, 2) interpersonal communication, 3) independence-execution, 4) independence-competence and 5) employment-occupation and allows a total score. The short form had a Cronbach' alpha of .76 and proved to be reliable for use in clinical practice. In the present study, we excluded employment related items and preferred to use a more objective measure (current employment status).

\section{Statistical analysis}

Statistical analyses were performed using SPSS version 25.0. Mean and standard deviation were used to evaluate descriptive data. Differences between the means of the two groups were evaluated through Student's $t$-test for two independent samples and Cohen's $d$ to calculate the effect size. Bivariate correlations were used to observe the relationships between attributional styles, 
clinical variables and SF within the clinical group. Multiple linear regressions with stepwise method were conducted to check possible predictors of clinical variables. The criterion was: probability of $F$ to enter $\leq .05$; probability of $F$ to eliminate $\geq .10$. Psychotic symptoms (PANSS scores) and delusion severity (PDI-21scores) were considered as dependent variables. Age and gender were forced to enter in the first step as possible confounding variables. In the second step, BDI-II and ASQ scores were introduced. In the case of Depression (BDI-II), same method was used in the first step. In the second step, PANSS scores were introduced. In the last step, ASQ scores were introduced. Finally, multiple linear regressions with the same stepwise method were conducted to check possible predictors of SF areas controlling by BDI-II (weighted least squares regression). To avoid possible collinearity between variables, only ASQ' composite indexes were included as possible predictors in the last step.

\section{Results}

Demographic and clinical characteristics of the participants are shown in Table 1.

Differences between groups in attributional styles

Statistically significant differences were found between both groups for most attributional styles with moderate to large effect sizes (Cohen's $d$ ). Table 2 shows differences between groups in attributional styles. The clinical group was significantly more "pessimistic" $(t=-5.69 ; p<.001)$. 
Table 1. Clinical and Demographic Characteristics of the Samples

\begin{tabular}{|c|c|c|c|c|c|}
\hline & & Clinical & Control & TOTAL & $t / z$ \\
\hline & & $M(S D)$ & $M(S D)$ & $M(S D)$ & \\
\hline \multirow[t]{2}{*}{ Age } & & $39.76(7.32)$ & 38 (10.73) & 38.80 (9.29) & 0.72 \\
\hline & & $N(\%)$ & $N(\%)$ & $N(\%)$ & \\
\hline \multirow[t]{2}{*}{ Gender } & Male & $22(40.7)$ & $16(29.1)$ & $38(69.8)$ & 1.28 \\
\hline & Female & $3(5.9)$ & $14(24.3)$ & $17(30.2)$ & \\
\hline \multirow[t]{3}{*}{ Education } & Elementary & $8(11.1)$ & $0(0)$ & $8(11.1)$ & $1.70^{*}$ \\
\hline & Middle & $11(19.4)$ & $9(16.7)$ & $20(36.1)$ & \\
\hline & University & $6(8.3)$ & $21(44.4)$ & $27(52.8)$ & \\
\hline \multirow[t]{3}{*}{ Marital status } & Single & $20(29.2)$ & $9(19.4)$ & $29(48.6)$ & $1.85^{*}$ \\
\hline & Partner & $4(6.9)$ & $19(37.5)$ & $23(44.4)$ & \\
\hline & Separated & $1(2.8)$ & $2(4.2)$ & $3(6.9)$ & \\
\hline \multirow[t]{4}{*}{ Employment } & Active/student & $2(2.8)$ & 27 (55.6) & $29(58.3)$ & $3.03^{*}$ \\
\hline & Unemployed & $7(9.7)$ & $2(4.2)$ & $9(13.9)$ & \\
\hline & Pensioner & $16(26.4)$ & $1(1.4)$ & $17(27.8)$ & \\
\hline & \multicolumn{3}{|c|}{$M(S D)$} & & \\
\hline \multicolumn{2}{|l|}{ PANSS Positive } & $15.08(5.02)$ & & & \\
\hline \multicolumn{2}{|l|}{ PANSS Negative } & $16.28(4.96)$ & & & \\
\hline \multicolumn{2}{|l|}{ PANSS Psychopath. } & $30.40(7.54)$ & & & \\
\hline \multicolumn{2}{|l|}{ PANSS Composite } & $-1.20(6.78)$ & & & \\
\hline \multicolumn{2}{|l|}{ PANSS TOTAL } & $61.76(14.00)$ & & & \\
\hline \multicolumn{2}{|l|}{ PDI-21 Stress } & $2.90(1.27)$ & & & \\
\hline \multicolumn{2}{|l|}{ PDI-21 Worry } & $2.64(1.22)$ & & & \\
\hline \multicolumn{2}{|l|}{ PDI-21 Conviction } & $2.71(1.20)$ & & & \\
\hline \multicolumn{2}{|l|}{ PDI-21 TOTAL } & $8.60(4.44)$ & & & \\
\hline \multicolumn{2}{|l|}{ BDI-II } & $11.96(5.76)$ & & & \\
\hline
\end{tabular}

Note. $z$ (Kolmogorov-Smirnoff). PANSS = Positive and Negative Syndrome Scale; PDI-21 = Peters Delusions Inventory; BDI-II = Beck Depression Inventory.

$* p<.01$.

Correlations between attributional styles and clinical variables (clinical group)

An unstable attribution tendency (for both negative and positive events) correlated with positive symptomatology (PANSS positive) and PANSS general psychopathology. Although no significant relation was found between any attributional style and negative symptoms, a stable attribution for negative events $(r=-.49 ; p<.01)$ and positive events $(r=-.37 ; p<.05)$ was significantly 
related to the predominance of negative symptomatology measured by PANSS composite (result not shown in tables).

Table 2. Comparison between Groups in the Attributional Styles (ASQ)

\begin{tabular}{lcccc}
\hline & Clinical $(n=25)$ & Control $(n=30)$ & $t$ & Cohen's $d$ \\
\hline Negative style & $12.91(2.53)$ & $10.48(1.87)$ & $4.09^{* * *}$ & 1.09 \\
Internal-External (-) & $4.23(0.96)$ & $4.26(0.65)$ & -0.10 & 0.04 \\
Stable-Unstable (-) & $4.52(1.13)$ & $3.36(0.89)$ & $4.19^{* * *}$ & 1.14 \\
Global-Specific (-) & $4.15(1.20)$ & $2.82(0.84)$ & $4.71^{* * *}$ & 1.28 \\
Positive style & $16.53(1.59)$ & $14.74(2.11)$ & $-3.60^{* *}$ & 1.49 \\
Internal-External (+) & $4.93(1.07)$ & $5.64(0.59)$ & $-3.01^{* *}$ & 0.82 \\
Stable-Unstable (+) & $5.14(0.79)$ & $5.59(0.50)$ & $-2.46^{*}$ & 0.68 \\
Global-Specific (+) & $4.67(1.01)$ & $5.20(0.91)$ & $-2.35^{*}$ & 0.65 \\
Composite index & $1.83(2.89)$ & $6.06(2.55)$ & $-5.69^{* * *}$ & 1.55 \\
Internal-external bias & $9.16(1.76)$ & $9.90(0.72)$ & -1.97 & 0.55 \\
Stable-unstable bias & $9.66(1.58)$ & $8.94(1.13)$ & 1.89 & 0.52 \\
Global-specific bias & $8.82(1.90)$ & $8.09(1.37)$ & 1.60 & 0.44 \\
Self-serving bias & $0.69(1.02)$ & $1.39(1.02)$ & $-2.52^{*}$ & 0.69 \\
\hline
\end{tabular}

Note. ASQ scores range from 1 to 7 . The closer to 7, the more indicative of an internal, stable and global attribution. (-) means "attribution for negative events". (+) means "attribution for positive events". The Composite index is a general indicator of "optimism-pessimism": lower scores suggest a general pessimistic trend.

${ }^{*} p<.05,{ }^{* *} p<.01,{ }^{* * *} p<.001$.

Regarding relationships between attribution and delusional beliefs, significant correlations were found between the severity of delusions (PDI-21 total score) and an external attribution for positive events, a positive attribution style (inversely), an externality bias and a pessimistic style (ASQ composite index).

A stable attribution for negative events and an external, unstable and specific attribution for positive events correlated with depression (BDI-II). A selfserving bias also correlated (inversely) with depression. A general "pessimistic" trend, measured by the ASQ' positive-negative composite index, highly correlated with depression $(r=-.642 ; p<.001)$. Correlations are shown in Table 3a. To 
demonstrate that relations between attributional styles and depression were not influenced by psychotic symptoms, partial correlations were calculated controlling by positive, negative and general symptoms. Correlations remained significant (see Table 3a).

Table 3a. Bivariate Correlations within the Clinical Symptom. Attribution Styles and Clinical Symptoms

\begin{tabular}{|c|c|c|c|c|c|c|c|c|c|}
\hline & $\begin{array}{l}\text { PDI } \\
\text { Total }\end{array}$ & Stress & Worry & $\begin{array}{c}\text { Convicti } \\
\text { on }\end{array}$ & Positive & Negative & General & BDI-II & BDI-II ${ }^{1}$ \\
\hline $\begin{array}{l}\text { Negative } \\
\text { style }\end{array}$ & .115 & .099 & -.045 & .109 & -.209 & -.020 & -.224 & .267 & .312 \\
\hline $\begin{array}{l}\text { Internal } \\
\text { negative }\end{array}$ & -.180 & .062 & .035 & .203 & -.253 & -.062 & -.132 & .032 & .148 \\
\hline $\begin{array}{c}\text { Stable } \\
\text { negative }\end{array}$ & .205 & .108 & -.097 & .023 & $-.370^{*}$ & .298 & -.290 & $.335^{*}$ & .339 \\
\hline $\begin{array}{c}\text { Global } \\
\text { negative }\end{array}$ & .195 & .058 & -.031 & .047 & .110 & -.274 & -.093 & .223 & .274 \\
\hline $\begin{array}{l}\text { Positive } \\
\text { style }\end{array}$ & $-.342^{*}$ & -.297 & -.212 & -.152 & -.093 & -.055 & -.016 & $-.561^{* *}$ & $-.557^{* * *}$ \\
\hline $\begin{array}{l}\text { Internal } \\
\text { positive }\end{array}$ & $-.433^{*}$ & -.201 & -.077 & -.011 & -.070 & .065 & .090 & $-.392^{*}$ & -.382 \\
\hline $\begin{array}{c}\text { Stable } \\
\text { positive }\end{array}$ & -.058 & -.215 & -.232 & -.141 & $-.426^{*}$ & .071 & -.316 & $-.383^{*}$ & $-.425^{*}$ \\
\hline $\begin{array}{c}\text { Global } \\
\text { positive }\end{array}$ & -.212 & -.249 & -.187 & -.201 & .221 & -.230 & .124 & $-.465^{* *}$ & $-.498^{*}$ \\
\hline $\begin{array}{c}\text { Composi } \\
\text { te }\end{array}$ & $-.350 *$ & -.303 & -.115 & -.206 & .115 & -.022 & .183 & $-.642^{* * *}$ & $-.682^{* * *}$ \\
\hline $\begin{array}{l}\text { Internal } \\
\text { bias }\end{array}$ & $-.361^{*}$ & -.087 & -.028 & .105 & -.181 & .005 & -.017 & -.220 & -.153 \\
\hline $\begin{array}{c}\text { Stable } \\
\text { bias }\end{array}$ & .117 & -.030 & -.185 & -.054 & $-.477^{* *}$ & .248 & $-.365^{*}$ & .048 & .018 \\
\hline $\begin{array}{c}\text { Global } \\
\text { bias }\end{array}$ & .010 & -.096 & -.119 & -.077 & .187 & -.296 & .008 & -.107 & -.094 \\
\hline $\begin{array}{l}\text { Self- } \\
\text { serving } \\
\text { bias }\end{array}$ & -.283 & -.269 & -.113 & -.204 & .166 & .126 & .219 & $-.441^{*}$ & $-.536^{* *}$ \\
\hline
\end{tabular}


Tabla 3b. Bivariate Correlations within the Clinical Symptom. Atrribution Styles and Clinical Symptoms with Social Functioning

\begin{tabular}{|c|c|c|c|c|c|c|}
\hline & Total & Isolation & $\begin{array}{c}\text { Communicati } \\
\text { on }\end{array}$ & $\begin{array}{l}\text { Independence } \\
\text {-Execution }\end{array}$ & $\begin{array}{l}\text { Independence } \\
\text {-Competence }\end{array}$ & Employment \\
\hline $\begin{array}{l}\text { PANSS } \\
\text { Positive }\end{array}$ & -.196 & .034 & .015 & -.265 & -.271 & -.014 \\
\hline $\begin{array}{l}\text { PANSS } \\
\text { Negative }\end{array}$ & -.202 & -.025 & -.109 & -.084 & -.307 & .001 \\
\hline $\begin{array}{l}\text { Psychopath } \\
\text { ology }\end{array}$ & $-.341^{*}$ & .041 & -.098 & $-.348^{*}$ & $-.418^{*}$ & -.116 \\
\hline BDI-II & .078 & -.279 & -.053 & .268 & .159 & .262 \\
\hline $\begin{array}{l}\text { Negative } \\
\text { Style }\end{array}$ & .292 & .057 & .007 & .307 & $.397^{*}$ & -.309 \\
\hline $\begin{array}{l}\text { Internal } \\
\text { Negative }\end{array}$ & .070 & .067 & -.128 & .106 & .221 & $-.417^{*}$ \\
\hline $\begin{array}{l}\text { Stable } \\
\text { Negative }\end{array}$ & .113 & -.021 & -.102 & .143 & $.330^{*}$ & -.082 \\
\hline $\begin{array}{l}\text { Global } \\
\text { Negative }\end{array}$ & $.453^{*}$ & .087 & .214 & $.429 *$ & $.350^{*}$ & -.240 \\
\hline $\begin{array}{l}\text { Positive } \\
\text { Style }\end{array}$ & .096 & $.379 *$ & .174 & -.085 & -.171 & $-.574^{* *}$ \\
\hline $\begin{array}{l}\text { Internal } \\
\text { Positive }\end{array}$ & -.161 & .135 & -.117 & -.106 & -.260 & $-.398^{*}$ \\
\hline $\begin{array}{l}\text { Stable } \\
\text { Positive }\end{array}$ & .137 & $.403^{*}$ & .255 & -.195 & -.034 & $-.362^{*}$ \\
\hline $\begin{array}{l}\text { Global } \\
\text { Positive }\end{array}$ & .258 & $.335^{*}$ & .292 & .077 & -.068 & $-.497^{* *}$ \\
\hline $\begin{array}{l}\text { Composite } \\
\text { Index }\end{array}$ & -.185 & .225 & .120 & $-.330 *$ & $-.471^{* *}$ & -.147 \\
\hline $\begin{array}{l}\text { Internal } \\
\text { bias }\end{array}$ & -.059 & .118 & -.141 & -.006 & -.036 & $-.470^{* *}$ \\
\hline Stable bias & .149 & .187 & .054 & .005 & .219 & -.239 \\
\hline Global bias & $.424^{*}$ & .234 & .291 & .312 & .185 & $-.416^{*}$ \\
\hline $\begin{array}{l}\text { Self-serving } \\
\text { bias }\end{array}$ & -.235 & .078 & -.002 & -.212 & $-.481^{* *}$ & -.022 \\
\hline
\end{tabular}

Note. PDI-21 = Total, Stress, Worry, and Conviction. PANSS positive symptoms, negative symptoms, and general psychopathology. ${ }^{1}$ BDI-II $=$ score. ${ }^{1}$ BDI-II: Partial correlations $(d f=20)$ controlling by PANSS positive, negative, and general psychopathology. SFS-R: Social Functional Scale. Total (general social functioning), social isolation, interpersonal communication, independence-execution, independencecompetence. Employment status (negative correlations mean better employment status).

${ }^{*} p<.05,{ }^{* *} p<.01,{ }^{* * *} p<.001$. 
Correlations between attributional styles and clinical symptoms with social functioning

General psychopathology inversely correlated with General SF, independence-execution and independence-competence. Correlations between clinical symptoms and attributional styles with SF areas are shown in Table $3 \mathrm{~b}$.

Correlations between educational level, employment, attributional styles and social functioning

A stable and global attribution for positive events correlated to educational level. The employment status was related to an internal, stable and global attribution for positive events, and with an internal attribution for negative events. Employment was also related with general SF. A higher educational level and a better employment situation correlated with a lower social isolation. The full correlation matrix is shown in Table 4.

Employment status was considered as a nominal variable $(1=$ study or work; 2 = unemployed; 3 = pensioner). For practical purposes we have considered condition 1 as the most satisfactory and condition 3 as the least satisfactory. Therefore, negative correlations would indicate a positive relationship.

\section{Multiple linear regressions}

A general "pessimistic" style predicted over $40 \%$ of the variance in depression, $F(3,21)=5,04 ; p=.009$.

An unstable attribution bias predicted more than $25 \%$ of the variance in positive psychotic symptoms, $F(3,21)=2.55: p=.08$. No model predicted adequately negative symptoms, general psychopathology, or delusion severity. 
Table 4. Correlation matrix.

\begin{tabular}{|c|c|c|c|c|c|c|c|c|c|c|c|c|c|c|c|c|c|c|c|c|c|c|c|c|c|c|c|c|}
\hline & 1 & 2 & 3 & 4 & 5 & 6 & 7 & 8 & 9 & 10 & 11 & 12 & 13 & 14 & 15 & 16 & 17 & 18 & 19 & 20 & 21 & 22 & 23 & 24 & 25 & 26 & 27 & 28 \\
\hline 1. Education & & .18 & -30 & .22 & -.11 & .12 & -.33 & .16 & -.01 & .12 & -30 & -19 & -.20 & $-.55^{* *}$ & -.33 & .11 & .21 & -.11 & .17 & $.44^{*}$ & 39 & .11 & $.42^{*}$ & .22 & $.35^{*}$ & -.02 & .33 & .21 \\
\hline 2. SFS Total & & & $.69^{* *}$ & $.65^{* *}$ & $.61^{* *}$ & $.57^{* *}$ & $-39^{*}$ & -.20 & -.20 & $-34^{*}$ & -.27 & -30 & -.26 & -.01 & .08 & .29 & .07 & .11 & $.45^{*}$ & .10 & -16 & 18 & .26 & -19 & -.06 & .15 & $.42^{*}$ & -.24 \\
\hline 3. Isolation & & & & $.59^{* *}$ & .12 & .20 & $-.50^{* *}$ & .03 & -.03 & .04 & $-.60^{* *}$ & $*-.55^{* *}$ & $-36^{*}$ & -.29 & -.28 & .06 & .07 & -.02 & .09 & $.38^{*}$ & .14 & $.40^{*}$ & $.34^{*}$ & .23 & .12 & 19 & .23 & .08 \\
\hline 4. Communica. & & & & & -.06 & -.02 & -.17 & .02 & -.11 & -.10 & -.15 & -.07 & -.11 & .00 & -.05 & .01 & -.13 & -.10 & .21 & $.17-$ & -.12 & .26 & .29 & .12 & -.14 & .05 & .29 & -.00 \\
\hline 5. Indep-Exe. & & & & & & $.44^{*}$ & -.20 & -.27 & -.08 & $-.35^{*}$ & -14 & -.23 & -19 & .07 & .27 & .31 & .12 & .14 & $.43^{*}$ & $-.09-$ & -.11 & -.20 & .08 & -.33 & -.01 & .01 & .31 & -.21 \\
\hline 6. Indep-Comp. & & & & & & & -.23 & -.27 & -.31 & $-42^{*}$ & .12 & -.03 & -.06 & .09 & 16 & $.40^{*}$ & .22 & .33 & $.35^{*}$ & $-.17 \quad-$ & -.26 & -.03 & -.07 & $-47^{* *}$ & -.04 & .22 & 19 & $-.48^{* *}$ \\
\hline 7. Employment & & & & & & & & -.01 & .00 & -.12 & $.48^{* *}$ & ${ }^{*} .31$ & .13 & .30 & .26 & -.31 & $-42^{*}-$ & $-.08-$ & -.24 & $-.57^{* *}$ & $-40^{*}$ - & $-36^{*}$ & $-.50^{* *}$ & $-.15-$ & $-.47^{* *}$ & -.24 & $-42^{*}$ & -.02 \\
\hline 8. PANSS P. & & & & & & & & & .08 & $.77^{* *}$ & -.27 & -.16 & .02 & -.07 & .18 & -.21 & $-.25-$ & $-\left.3\right|^{*}$ & .11 & $-.09-$ & $-.07-$ & $-43^{*}$ & .22 & .12 & -.18 & $-48^{* *}$ & .19 & .17 \\
\hline 9. PANSS N. & & & & & & & & & & $.37^{*}$ & -.16 & -15 & -.17 & -.04 & $.28-$ & -.02 & -.06 & .30 & -.27 & -.06 & .07 & .07 & -.23 & -.02 & .01 & .25 & -30 & .13 \\
\hline 10. Psychop. & & & & & & & & & & & -.23 & -.07 & .05 & -.29 & .06 & -.22 & -.13 & $-.29-$ & -.09 & -.02 & .09 & -.32 & .12 & .18 & $-.02-$ & $.37^{*}$ & .01 & .22 \\
\hline 11. PDI Stress & & & & & & & & & & & & $.90^{* *}$ & $.69^{* *}$ & $.46^{*}$ & $.40^{*}$ & .10 & .06 & .11 & .06 & $-30-$ & $-.20-$ & -.22 & -.25 & -.30 & -.09 & -.03 & -10 & -.27 \\
\hline 12. PDI Conc. & & & & & & & & & & & & & $.77^{* *}$ & $.34^{*}$ & $.35^{*}-$ & -.05 & .04 & -.10 & -.03 & -.21 & $-.08-$ & -.23 & -.19 & -.12 & -.03 & -.19 & -.12 & -.11 \\
\hline 13. PDI Conv. & & & & & & & & & & & & & & .30 & $.41^{*}$ & .11 & .21 & .02 & .10 & $-15-$ & $-.01-$ & -.14 & -.20 & -.21 & .11 & -.05 & -.11 & $-.44^{*}$ \\
\hline 14. PDI Total & & & & & & & & & & & & & & & $.41^{*}$ & .12 & -18 & .21 & .20 & $-.34^{*}-$ & $-.43^{*}-$ & -.06 & -.21 & $-.35^{*}-$ & -.36 & .12 & .01 & -.28 \\
\hline 15. BDI-II & & & & & & & & & & & & & & & & .27 & .03 & $.34^{*}$ & .22 & $-.56^{* *}$ & $-39^{*}$ & $-38^{*}$ & $-.47^{*}$ & $-64^{* *}$ & -.22 & .05 & -.11 & $-.44^{*}$ \\
\hline 16. Neg. style & & & & & & & & & & & & & & & & & $.76^{* *}$ & $.79^{* *}$ & $.76^{* *}$ & .23 & .06 & .28 & .19 & $-.71^{* *}$ & $.45^{*}$ & $.70^{* *}$ & $.58^{* *}$ & $*^{*}-.65^{* *}$ \\
\hline 17. Internal (-) & & & & & & & & & & & & & & & & & & $.47^{* *}$ & $.35^{*}$ & $.52^{* *}$ & $.50^{* *}$ & $.43^{*}$ & .21 & -.28 & $.85^{* *}$ & $.55^{* *}$ & .33 & $-.42^{*}$ \\
\hline 18. Stable (-) & & & & & & & & & & & & & & & & & & & $.35^{*}$ & -.08 & -.17 & $.34^{*}$ & -.26 & $-.75^{* *}$ & .16 & $.88^{* *}$ & $.00^{* *}$ & $*-45^{*}$ \\
\hline 19. Global (-) & & & & & & & & & & & & & & & & & & & & .14 & -.11 & -.08 & $.47^{* *}$ & $-.56^{* *}$ & .12 & .21 & & $*-.45^{*}$ \\
\hline 20. Pos. style & & & & & & & & & & & & & & & & & & & & & $.84^{* *}$ & $.58^{* *}$ & $.76^{* *}$ & $.53^{* *}$ & $.79^{* *}$ & .23 & $.49^{* *}$ & * $.39^{*}$ \\
\hline 21. Internal $(+)$ & & & & & & & & & & & & & & & & & & & & & & .29 & $.47^{* *}$ & $.56^{* *}$ & $.88^{* *}$ & .03 & 18 & $.57^{* *}$ \\
\hline 22. Stable $(+)$ & & & & & & & & & & & & & & & & & & & & & & & .11 & .18 & $.41^{*}$ & $.74^{* *}$ & .01 & -.10 \\
\hline 23. Global $(+)$ & & & & & & & & & & & & & & & & & & & & & & & & $.39^{*}$ & $.40^{*}$ & -.13 & $.83^{* *}$ & $* .30$ \\
\hline 24. Pessimism & & & & & & & & & & & & & & & & & & & & & & & & & .18 & $-.45^{*}$ & -.15 & $.85^{* *}$ \\
\hline 25. Inter. bias & & & & & & & & & & & & & & & & & & & & & & & & & & .32 & .29 & .12 \\
\hline 26. Stab. bias & & & & & & & & & & & & & & & & & & & & & & & & & & & .6 & $-.50^{* *}$ \\
\hline 27. Glob. bias & & & & & & & & & & & & & & & & & & & & & & & & & & & & -.12 \\
\hline 28. Self-s. bias & & & & & & & & & & & & & & & & & & & & & & & & & & & & \\
\hline
\end{tabular}

Note. 1. Educational level; 2. General social functioning; 3. Social isolation; 4. Interpersonal communication; 5. Independence-execution; 6. Independence-competence; 7. Employment status; 8. PANSS positive; 9. PANSS negative; 10. PANSS general psychopathology; 11. PDI-21 stress; 12 . PDI-21 worry; 13. PDI-21 conviction; 14. PDI-21 Total; 15. BDI-II; 16 . Negative attribution style; 17. Internal negative; 18. Stable negative; 19. Global negative; 20. Positive attribution style; 21. Internal positive; 22. Stable positive; 23. Global positive; 24. Pessimistic style; 25. Internal bias; 26. Stable bias; 27. Global bias; 28. Self-serving bias.

$* p<.05 . * * p<.01$.

Regarding SF, after weighting for depression, PANSS general psychopathology predicted a worse general SF and a global attribution style predicted a better one, $F(4,20)=4.49 ; p=.009$. A global attribution predicted better Interpersonal communication, $F(3,21)=2.40 ; p=.09$. General psychopathology predicted a worse independence-execution, $F(3,21)=4.39$; $p$ $=.015$, and a worse independence-competence, $F(3,21)=4.19 ; p=.018$. A positive attribution style predicted a better employment status, $F(3,21)=5.72 ; p$ $=.005$. No model predicted adequately social isolation. Table 5 summarizes all regression models that were predictive. 
Table 5. Summary of Regression Models

\begin{tabular}{|c|c|c|c|c|c|c|}
\hline \multicolumn{7}{|c|}{ Predicted variable: Depression (BDI-II) } \\
\hline Model & Predictors & $B$ & $\beta$ & $R^{2}$ & Change in $R^{2}$ & $p$-value \\
\hline 2 & Pessimism $^{1}$ & -1.25 & -.63 & .419 & .369 & .001 \\
\hline \multicolumn{7}{|c|}{ Predicted variable: Positive symptoms (PANSS positive) } \\
\hline Model & Predictors & $B$ & $\beta$ & $R^{2}$ & Change in $R^{2}$ & $p$-value \\
\hline 2 & Stable bias & -1.54 & -.49 & .267 & .231 & .018 \\
\hline \multicolumn{7}{|c|}{ Predicted variable: General Social Functioning² } \\
\hline \multirow{3}{*}{$\begin{array}{l}\text { Model } \\
3\end{array}$} & Predictors & $B$ & $\beta$ & $R^{2}$ & Change in $R^{2}$ & $p$-value \\
\hline & Psychopathology & -0.25 & -.39 & .291 & .193 & .026 \\
\hline & Global attribution & 1.08 & .44 & .473 & .182 & .016 \\
\hline \multicolumn{7}{|c|}{ Predicted variable: Interpersonal communication ${ }^{2}$} \\
\hline Model & Predictor & $B$ & $\beta$ & $R^{2}$ & Change in $R^{2}$ & $p$-value \\
\hline 2 & Global attribution & -0.60 & .49 & .255 & .224 & .020 \\
\hline \multicolumn{7}{|c|}{ Predicted variable: Independence-execution ${ }^{2}$} \\
\hline \multirow{3}{*}{$\begin{array}{l}\text { Model } \\
2\end{array}$} & Predictors & $B$ & $\beta$ & $R^{2}$ & Change in $R^{2}$ & $p$-value \\
\hline & Gender & 2.64 & .44 & .385 & .171 & .019 \\
\hline & Psychopathology & -0.13 & -.41 & & & .025 \\
\hline \multicolumn{7}{|c|}{ Predicted variable: Independence-competence ${ }^{2}$} \\
\hline Model & Predictor & B & $\beta$ & $R^{2}$ & Change in $R^{2}$ & $p$-value \\
\hline 2 & Psychopathology & -0.10 & -.51 & .375 & .254 & .008 \\
\hline \multicolumn{7}{|c|}{ Predicted variable: Employment status ${ }^{2}$} \\
\hline Model & Predictors & $B$ & $\beta$ & $R^{2}$ & Change in $R^{2}$ & $p$-value \\
\hline 2 & Positive style & -0.15 & -.45 & .450 & .182 & .016 \\
\hline
\end{tabular}

Note. $B=$ non standardized coefficients; $\beta=$ standardized coefficients. All models included contributions of age and gender to the prediction. ${ }^{1}$ Pessimism = ASQ' Composite positive-negative index. ${ }^{2}$ Weighted least squares regression by BDI-II.

\section{Discussion}

Results provide evidence for the utility and adequacy of both reformulated Learned Helplessness Model (Abramson et al., 1978) and its more fully articulated form, the Hopelessness Theory of Depression (Davidson et al., 2018; Haeffel et al., 2017), to predict depression in persons with schizophreniaspectrum disorders. Comparing to a non-clinical group, persons with psychosis show an increased negative attribution bias. Moreover, the clinical group shows a 
"pessimistic" attribution bias as an important differential trait comparing to controls, with a great effect size. The finding agrees with some studies linking optimism to better mental health levels (Yuan \& Wang, 2016). Within the clinical group, "pessimism" predicted more than $40 \%$ of the variance in depressive symptoms according to predictions of the Theory of Hopelessness of Depression (Haeffel et al., 2017). Although our results demonstrate the hypothesis that people with psychotic disorders have a "depressive" attribution style, we find no difference in the internal attribution for negative situations. The internal-external dimension has shown low-reliability coefficients, which could be due to a problem of consistency of internality attributions itself (Sanjuán et al., 2013) and should be considered when interpreting the results of this and other studies.

Interestingly, in the current study, an abolished "self-serving bias" (a tendency to internalize blame for negative events) (Moritz et al., 2007), is related to depressive symptoms and to a pessimistic style. Other previous works find the opposite pattern in persons with paranoid delusions, including an excessive tendency to attribute positive events to oneself and negative events to external causes, indicating an increased "self-serving bias" (Candido \& Romney, 1990; cited by Moritz et al., 2018). Additionally, to demonstrate that relations found between a "depressive" attributional style and depression severity are not influenced by psychotic symptoms, we calculated partial correlations controlling by positive, negative and general psychopathology, and results remained significant. This could be an important finding to support our hypothesis about the suitability of the Learned helplessness model to predict depression in persons with psychosis, regardless of psychotic symptoms.

Regarding delusions, some studies employing the ASQ provide support for the hypothesis that delusional persons show an externality bias when attributing causes for negative events, although internal attributions for positive events are not found (Aakre et al., 2009). This bias is more linked to psychosis in general (e.g., Jolley et al., 2006), while more recent studies find that persons who tend to explain negative events by external causes are more specifically prone to delusional beliefs about such experiences (Langdon et al., 2013). In our study, it 
is the tendency to view life events as the result of unstable or unpredictable causes (unstable attributional bias) and not an externalising bias for negative events, which better predicts the severity of positive symptoms, according to our hypothesis and to Lysaker et al.'s (2004) findings. Some studies find increased helplessness (a bias to externalise both positive and negative events) in psychotic patients (Lincoln et al., 2010). An external attribution bias for positive events (but not for negative ones) is related to the severity of delusional beliefs, which is partially in line with these previous studies (Achim et al., 2016; So et al., 2015).

The belief that the causes of both positive and negative events are generalisable and relate to many areas of our lives (a global attribution style) predicts a better general SF and better interpersonal communication in people with psychotic symptoms. However, contrary to Lysaker et al. (2004), negative symptoms are not related to a worse $\mathrm{SF}$, but an unstable attribution bias is significantly related to the predominance of negative symptoms. Higher scores in general psychopathology also predict lower independence for daily life activities. A higher educational level, a better employment status and a stable attribution for positive situations are related to less social isolation. Persons with psychosis who believe that the positive events of their lives are stable and predictable, enjoy better social relationships and are less isolated, according to Lysaker et al. (2004) that suggest a stable attribution as the most related style to different measures of SF.

Finally, people with psychosis who have a positive attributional style (internal, stable and global attribution for positive situations) are more likely to remain active.

The results presented here seek to update classical studies based on the theory of attribution from the learned helplessness model and should be put in the context of recent research. So, Moritz et al. (2018) notice that, despite the heterogeneity of attributional styles, there is growing evidence to suggest that persons with schizophrenia share a tendency to "monocausal" or one-sided attributions: they do not spread the potential causes for events across different factors ("myself", other persons or circumstances), confine the causal search to 
one predominant source (Mehl et al., 2014; Moritz et al., 2015) and is related to a widely studied reasoning bias such as the "jumping to conclusions" (Moritz et al., 2018). The attributional style seems more "a thinking style" related to personality than a social cognition domain and it's associated with paranoid traits and not exactly with psychotic symptoms (Savla et al., 2013). Some people in the early stages of the disease are characterised by a paranoid or hostile cognitive style that could be considered as a risk factor for developing delusional belief systems (Garety \& Freeman, 2013) and predisposes individuals to develop a psychotic disorder (Healey et al., 2016). Cognitive-behavioural interventions and others addressed to social cognition and reasoning skills have demonstrated that changes in these domains could improve symptoms and SF in psychiatric patients (Horan \& Green, 2019; Javed \& Charles, 2018).

Our study presents some limitations. First, the small sample size does not allow us to easily generalise the results. Also, a significant portion of clinical subjects was long-term patients treated in psychosocial rehabilitation services, so young patients or those with early psychosis would be underrepresented in the sample. Similarly, since we aimed to test the validity of the Hopelessness Theory of Depression, a group with depression disorders would have been more suitable as a control. Another limitation is the cross-sectional study design that hinders the determination of causal relationships since it is impossible to know whether the attribution styles that patients relate preceded the onset of the symptoms and influenced SF outcomes or, on the contrary, the symptoms modified the attribution styles at the present moment. Given that persecutory delusions are especially related to some attributional styles, another problem of our study is the impossibility to compare the differences between delusional and nondelusional participants due to the small sample size. We partially improved this limitation by relating attributional styles to delusion dimensions assessed by the PDI-21.

Finally, future research based on longitudinal design studies and more appropriate samples could improve some of these limitations, as well as more ecological assessment methods to avoid the shortcomings of the attributional 
style self-report measures. These future studies should attempt to address the issue of how attribution biases relate to the development of psychosis and their interaction with social cognition and other psychological or environmental factors, such as attachment experiences or childhood trauma, to predict SF in persons with schizophrenia-spectrum disorders.

The reformulated Learned Helplessness model provides utility to predict depression in persons with schizophrenia-spectrum disorders regardless of psychotic symptoms. The mechanism seems to involve a "pessimistic" bias towards the interpretation of life events. An unstable attribution bias (but not an externalising one) results more predictive to explain positive symptoms and a global attribution style predicts a better social functioning in persons with psychotic disorders. Recent models highlighting the existence of a "monocausal" attribution of events seem to be more adequate to understand the mechanisms of delusions through reasoning biases and could be a risk factor to develop psychosis. How attribution biases and impairments in cognition dimensions interact modifying social functioning remain unclear. If attribution biases and other social cognition domains are involved in the formation of psychotic symptoms and predict social functioning, their inclusion as a core target within psychological intervention programs would be mandatory. Moreover, since hopelessness predicts a worse global functioning and is a risk factor for suicidal behaviour, its assessment must be an essential issue to protect and promote the recovery of persons with schizophrenia-spectrum disorders.

\section{Conflicts of interest}

All authors declare no conflict of interests.

\section{References}

Aakre, J. M., Seghers, J. P., St-Hilaire, A., \& Docherty, N. (2009). Attributional style in delusional patients: A comparison of remitted paranoid, remitted nonparanoid, and 
current paranoid patients with nonpsychiatric controls. Schizophrenia Bulletin, 35(5), 994-1002. https://doi.org/10.1093/schbul/sbn033

Abramson, L. Y., Seligman, M. E., \& Teasdale, J. D. (1978). Learned helplessness in humans: Critique and reformulation. Journal of Abnormal Psychology, 87(1), 49-74. https://doi.org/10.1037/0021-843X.87.1.49

Achim, A. M., Sutliff, S., Samson, C., Montreuil, T. C., \& Lecomte, T. (2016). Attribution bias and social anxiety in schizophrenia. Schizophrenia Research: Cognition, 4, 1-3. https://doi.org/10.1016/j.scog.2016.01.001

Alonso, J., Olivares, J. M., Ciudad, A., Manresa, J. M., Casado, A., \& Gilaberte, I. (2008). Desarrollo y validación de la versión corta de la Escala de Funcionamiento Social en esquizofrenia para su uso en la práctica clínica. Actas Espanolas de Psiquiatria, 36(2), 102-110.

American Psychiatric Association (2013). Diagnostic and Statistical Manual of Mental Disorders (5th ed.). Arlington, VA: American Psychiatric Association.

Beck, A. T., Steer, R. A., \& Brown, G. K. (1996). Manual for the Beck depression inventoryII. San Antonio, TX: Psychological Corporation.

Bentall, R. P., Corcoran, R., Howard, R., Blackwood, N., \& Kinderman, P. (2001). Persecutory delusions: A review and theoretical integration. Clinical Psychology Review, 21, 1143-1192. https://doi.org/10.1016/S0272-7358(01)00106-4

Berardelli, I., Sarubbi, S., Rogante, E., Hawkins, M., Cocco, G., Erbuto, D., Lester, D., \& Pompili, M. (2019). The Role of Demoralization and Hopelessness in Suicide Risk in Schizophrenia: A Review of the Literature. Medicina, 55(5), 200. https://doi.org/ 10.3390/medicina55050200

Buck, B. E., Healey, K. M., Gagen, E. C., Roberts, D. L., \& Penn, D. L. (2016). Social cognition in schizophrenia: factor structure, clinical and functional correlates. Journal of Mental Health, 25, 330-337. https://doi.org/10.3109/09638237.2015.1124397

Candido, C. L., \& Romney, D. M. (1990). Attributional style in paranoid vs. depressed patients. British Journal of Medical Psychology, 63(4), 355-363. https://doi.org/ 10.1111/j.2044-8341.1990.tb01630.x

Cassidy, R. M., Yang, F., Kapczinski, F., \& Passos, I. C. (2018). Risk Factors for Suicidality in Patients With Schizophrenia: A Systematic Review, Meta-analysis, and Meta- 
regression of 96 Studies. Schizophrenia bulletin, 44(4), 787-97. https://doi.org/ $10.1093 /$ schbul/sbx131

Davidson, C. A., Lesser, R., Parente, L. T., \& Fiszdon, J. M. (2018). Psychometrics of social cognitive measures for psychosis treatment research. Schizophrenia Research, 193, 51-57. https://doi.org/10.1016/J.SCHRES.2017.06.018

Garety, P. A., \& Freeman, D. (2013). The past and future of delusions research: From the inexplicable to the treatable. British Journal of Psychiatry, 203, 327-333. https:// doi.org/10.1192/bjp.bp.113.126953

Haeffel, G. J., Hershenberg, R., Goodson, J. T., Hein, S., Square, A., Grigorenko, E. L., \& Chapman, J. (2017). The Hopelessness Theory of Depression: Clinical Utility and Generalizability. Cognitive Therapy and Research, 41, 543-555. https://doi.org/ $10.1007 / \mathrm{s} 10608-017-9833-1$

Healey, K. M., Bartholomeusz, C. F., \& Penn, D. L. (2016). Deficits in social cognition in first episode psychosis: A review of the literature. Clinical Psychology Review, 50, 108137. https://doi.org/10.1016/J.CPR.2016.10.001

Horan, W. P., \& Green, M. F. (2019). Treatment of social cognition in schizophrenia: Current status and future directions. Schizophrenia Research, 203, 3-11. https:// doi.org/10.1016/j.schres.2017.07.013

Howes, O. D., \& Murray, R. M. (2014). Schizophrenia: an integrated sociodevelopmentalcognitive model. Lancet, 383(9929), 1677-87. https://doi.org/10.1016/ S0140-6736(13)62036-X

Javed, A., \& Charles, A. (2018). The importance of social cognition in improving functional outcomes in schizophrenia. Frontiers in Psychiatry, 9, 1-14. https:// doi.org/10.3389/fpsyt.2018.00157

Jolley, S., Garety, P., Bebbington, P., Dunn, G., Freeman, D., Kuipers, E., ... Hemsley, D. (2006). Attributional style in psychosis-The role of affect and belief type. Behaviour Research and Therapy, 44(11), 1597-1607. https://doi.org/10.1016/j.brat. 2005.12.002

Kay, S. R., Fiszbein, A., \& Opler, L. A. (1987). The positive and negative syndrome scale (PANSS) for schizophrenia. Schizophrenia Bulletin, 13, 261-276. https://doi.org/ $10.1093 /$ schbul/13.2.261 
Langdon, R., Still, M., Connors, M. H., Ward, P. B., \& Catts, S. V. (2013). Attributional biases, paranoia, and depression in early psychosis. British Journal of Clinical Psychology, 52(4), 408-423. https://doi.org/10.1111/bjc.12026

Lincoln, T. M., Mehl, S., Exner, C., Lindenmeyer, J., \& Rief, W. (2010). Attributional style and persecutory delusions. Evidence for an event independent and state specific external-personal attribution bias for social situations. Cognitive Therapy and Research, 34(3), 297-302. https://doi.org/10.1007/s10608-009-9284-4

Liu, R. T., Kleiman, E. M., Nestor, B. A., \& Cheek, S. M. (2015). The Hopelessness Theory of Depression: A Quarter-Century in Review. Clinical Psychology: Science and Practice, 22(4), 345-365. https://doi.org/10.1111/cpsp.12125

López-Ilundain, J. M., Pèrez-Nievas, F., Otero, M., \& Mata, I. (2006). Peters delusions inventory in Spanish general population: Internal reliability, factor structure and association with demographic variables (dimensionality of delusional ideation). Actas Espanolas de Psiquiatria, 34(2), 94-104.

Lysaker, P. H., Lancaster, R. S., Nees, M. A., \& Davis, L. W. (2004). Attributional style and symptoms as predictors of social function in schizophrenia. Journal of Rehabilitation Research and Development, 41(2), 225-232. https://doi.org/10.1682/JRRD. 2004.02.0225

Mehl, S., Landsberg, M. W., Schmidt, A. C., Cabanis, M., Bechdolf, A., Herrlich, J., ... Wagner, M. (2014). Why do bad things happen to me? Attributional style, depressed mood, and persecutory delusions in patients with schizophrenia. Schizophrenia Bulletin, 40, 1338-1346. https://doi.org/10.1093/schbul/sbu040

Moritz, S., Bentall, R. P., Kolbeck, K., \& Roesch-Ely, D. (2018). Monocausal attribution and its relationship with reasoning biases in schizophrenia. Schizophrenia Research, 193, 77-82. https://doi.org/10.1016/j.schres.2017.06.057

Moritz, S., Köther, U., Hartmann, M., \& Lincoln, T. M. (2015). Stress is a bad advisor. Stress primes poor decision making in deluded psychotic patients. European Archives of Psychiatry and Clinical Neuroscience, 265, 461-469. https://doi.org/10.1007/ s00406-015-0585-1

Moritz, S., Woodward, T. S., Burlon, M., Braus, D. F., \& Andresen, B. (2007). Attributional style in schizophrenia: Evidence for a decreased sense of self-causation in currently 
paranoid patients. Cognitive Therapy and Research, 31(3), 371-383. https://doi.org/ 10.1007/s10608-006-9070-5

Murphy, P., Bentall, R. P., Freeman, D., O'Rourke, S., \& Hutton, P. (2018). The paranoia as defence model of persecutory delusions: a systematic review and meta-analysis. The Lancet Psychiatry, 5(11), 913-29. https://doi.org/10.1016/S2215-0366(18)30339-0

Peralta, V., \& Cuesta, M. J. (1994). Psychometric properties of the Positive and Negative Syndrome Scale (PANSS) in schizophrenia. Psychiatry Research, 53(1), 31-40. https:// doi.org/10.1016/0165-1781(94)90093-0

Peters, E., Joseph, S., Day, S., \& Garety, P. (2004). Measuring delusional ideation: The 21item Peters et al. Delusions Inventory (PDI). Schizophrenia Bulletin, 30, 1005-1022. https://doi.org/10.1093/oxfordjournals.schbul.a007116

Peterson, C., Semmel, A., von Baeyer, C., Abramson, L. Y., Metalsky, G. I., \& Seligman, M. E. P. (1982). The attributional Style Questionnaire. Cognitive Therapy and Research, 6, 287-299. https://doi.org/10.1007/BF01173577

Saffarian, Z., Doulatshahi, B., Pourshabaz, A., \& Kerdar, N. R. (2018). The role of social cognition in predicting the social functioning in patients with chronic schizophrenia. Iranian Rehabilitation Journal, 16(3), 239-246. https://doi.org/10.32598/irj. 16.3.239

Sanjuán, P., Fraguas, D., Magallares, A., \& Merchán-Naranjo, J. (2009). Depressive symptomatology and attributional style in patients with schizophrenia. Clinical Schizophrenia and Related Psychoses, 3, 31-38. https://doi.org/10.3371/CSRP.3.1.3

Sanjuán, P., Magallares, A., González, J. L., \& Pérez-García, A. (2013). A study on the validity of the Spanish version of the Attributional Style Questionnaire for negative situations. Revista de Psicopatologia y Psicologia Clinica, 18(1), 61-71. https:// doi.org/10.5944/rppc.vol.18.num.1.2013.12763

Sanz, J., Perdigón, A. L., \& Vázquez, C. (2003). [Spanish adaptation of the Beck-II Depression Inventory (BDI-II): 2. Psychometric properties in the general population]. Clinica y Salud, 14, 249-280.

Savla, G. N., Vella, L., Armstrong, C. C., Penn, D. L., \& Twamley, E. W. (2013). Deficits in domains of social cognition in schizophrenia: A meta-analysis of the empirical evidence. Schizophrenia Bulletin, 9(5), 979-992. https://doi.org/10.1093/schbul/ sbs080 
So, S. H. W., Tang, V., \& Leung, P. W. L. (2015). Dimensions of Delusions and Attribution Biases along the Continuum of Psychosis. PLoS ONE, 10(12). https://doi.org/ 10.1371/journal.pone.0144558

Torres, A., \& Olivares, J. M. (2005). Validation of the Spanish version of the social functioning scale. Actas Espanolas de Psiquiatria, 33(4), 216-220. 


\section{Capítulo 6 Estudio 2}

\section{Las dimensiones del apego como predictores del funcionamiento social en la esquizofrenia ${ }^{4}$}

\section{Resumen}

Los patrones de apego y las adversidades en la infancia son relevantes para entender la vulnerabilidad y el desarrollo de la psicosis. El objetivo del estudio fue probar si el CAMIR, puede diferenciar los estilos de apego comparando un grupo de pacientes psicóticos con una muestra no clínica. Se hipotetizó que las dimensiones del apego predecirían el funcionamiento social (FS) dentro del grupo clínico. 79 personas fueron evaluadas con el CAMIR-R. El alfa de Cronbach se utilizó para probar la fiabilidad y las pruebas $t$ para evaluar diferencias entre grupos. Se ejecutaron múltiples regresiones lineales y análisis de mediación para explorar los posibles predictores del FS. Los resultados mostraron un mayor apego inseguro en el grupo clínico (13.8 \% versus $33.3 \%$ ). La psicopatología y la interferencia parental pronosticaron el FS general $\left(R^{2}=0.30 ; p<0.05\right)$ y la preocupación familiar predijo mayor aislamiento social $\left(R^{2}=0.19 ; p<0.02\right) \mathrm{y}$ peor situación ocupacional $\left(R^{2}=0.45 ; p<0.01\right)$. Permisividad parental $\mathrm{y}$ traumatismo infantil predijeron peor nivel educativo $\left(R^{2}=0.31 ; p<0.05\right)$. Los análisis de mediación revelaron un efecto directo de las dimensiones del apego sobre el FS, independientemente de los síntomas. En suma, el CAMIR-R resultó fiable para evaluar el apego en psicosis. Las personas con trastornos psicóticos muestran importantes déficits en FS y mayor prevalencia de apego inseguro. Varias dimensiones del apego predicen áreas específicas del FS, independientemente de la gravedad de los síntomas.

\footnotetext{
4 El presente estudio ha sido enviado para publicación a la revista Actas de Psiquiatría Española y se encuentra en proceso de revisión.
} 


\title{
Attachment Dimensions predict Social Functioning in persons with Schizophrenia-Spectrum Disorders, regardless of Symptom Severity
}

\author{
Cristina Monfort-Escrig ${ }^{1}$ and Josep Pena-Garijo ${ }^{1,2}$ \\ 1 Jaume I University. Castellon de la Plana (Spain); ${ }^{2}$ Mental Health Service. University Hospital \\ Doctor Peset. Valencia (Spain).
}

\section{Summary}

Introduction: Attachment patterns and early-life adversities are relevant to understand the role of psychosocial factors in the vulnerability and the development of psychosis. The first aim of the study was to test whether a dimensional attachment instrument, the CAMIR (from French; Cartes: Modèles Individuels de Relation), may differentiate attachment styles by comparing a group of psychotic patients with a non-clinical sample. Also, we hypothesised that attachment dimensions would predict Social Functioning (SF) within the clinical group.

Methods: Seventy-nine persons were assessed by the reduced Spanish version (CAMIR-R). We used Cronbach' alpha to test reliability. A $t$-test was performed to assess differences between groups. Multiple linear regressions and Mediation analyses were conducted within the clinical group to explore attachment dimensions as possible predictors of SF.

Results: A more insecure attachment was found in the clinical group (73.8\% versus 33.3\%). Attachment dimensions differentiated between both samples with large effect sizes. Psychopathology and parental interference predicted general SF $\left(R^{2}=0.30 ; p<0.05\right)$ as family concern predicted more social isolation $\left(R^{2}=\right.$ $0.19 ; p=0.02)$ and worse employment status $\left(R^{2}=0.45 ; p<0.01\right)$. Parental permissiveness and child traumatism inversely predicted educational level $\left(R^{2}=\right.$ 
$0.31 ; p<0.05)$. Mediation analyses revealed that attachment dimensions were linked to SF regardless of symptoms.

Conclusions: CAMIR-R was reliable to assess attachment in psychosis. Persons with schizophrenia-spectrum disorders show a significant impaired SF and a higher prevalence of insecure attachment. Several attachment dimensions predict specific areas of SF, regardless of symptom severity.

Keywords: Schizophrenia, psychosis, CAMIR-R, attachment, social functioning, mediation analysis.

\section{Introduction}

Attachment patterns and early life adversities are relevant to understand the role of psychosocial and environmental factors in the vulnerability and the development of psychosis ${ }^{1,2}$.The attachment was conceptualized as the "propensity to make intimate emotional bonds to particular individuals as a basic component of human nature"3. Attachment theory suggests that early experiences with caregivers become internalized as cognitive-affective representations or internal working models of the self and others; these models serve as templates for future relationships across the life course ${ }^{4}$. Some authors propose that this model provides a robust theoretical framework for understanding the impact of distressing or traumatic early personal experiences through a distortion of mental schemes, affective deregulation and alteration of interpersonal patterns 5 . According to Rajkumar' "attachment-developmentalcognitive" hypothesis of schizophrenia ${ }^{6}$, the attachment may also represent a construct capable of bridging developmental, neurobiological and interpersonal levels of analysis in psychosis 7,8 .

Most research shows that patients with schizophrenia tend to have an insecure attachment style ${ }^{1}$. The associations with symptom dimensions are not clear. Some studies have shown associations between anxious attachment and positive symptoms, as well as avoidant attachment and both positive and 
negative symptoms ${ }^{9}$, while no associations were found with the dismissing style ${ }^{10}$. Attachment measures correlate with psychotic phenomena, including paranoia ${ }^{11}$ and distress when hearing voices ${ }^{12}$. Moreover, attachment measures correlate with attributional biases ${ }^{13}$ and mentalizing 14 ; social, community functioning and interpersonal problems ${ }^{15}$; social cognition as a mediator between insecure attachment and clinical and functioning problems ${ }^{8}$, and to psychotic-like experiences ${ }^{16}$. Furthermore, some investigations have likewise suggested that secure adult attachment may be related to how individuals with psychosis engage with mental health services ${ }^{17,18}$.

Beyond the predominance of insecure attachment patterns in psychotic patients ${ }^{19}$, it is commonly admitted that childhood trauma has a significant impact in the development of psychotic experiences, mainly hallucinations and delusions ${ }^{1,20}$, and in the development of social cognitive abilities, which may represent a mediator between early life adversities and later symptom severity ${ }^{21}$. Further, psychological factors are related to insecure attachment and recognized neurobiological underpinnings $22-27$.

A final important issue regards how attachment theory has generated several tools for assessing adult attachment 28 . Different measures evaluate different levels of conscious awareness concerning attachment representations: typically, narrative instruments such as the Adult Attachment Interview ${ }^{29}$ facilitate the evaluation of non-conscious aspects of attachment via the external coding of responses. At the same time, self-report questionnaires focused on conscious ideas about attachment and relationships ${ }^{30}$. Self-report instruments are easier and more economical to use but can be over-simplistic, especially about unconscious representations as in the case of fearful attachment ${ }^{30}$. The literature agrees that both types of measures are useful, each serving to evaluate different kinds of construct ${ }^{30}$. In the current study, our interest focused on the CAMIR (from French; Cartes: Modèles Individuelles de Relation), a self-report instrument designed to tap into the relational strategies of adults ${ }^{31}$. The CAMIR's merit lies in the richness and complexity of the theoretical model underpinning it: a self-and-other model drives personal relationships throughout life and 
adulthood generated by early experience with caregivers. However, it may be modified by subsequent relational experiences ${ }^{30}$. It can be administered regardless of the subject' family structure; provides continuous measurements of attachment dimensions, that allows evaluating clinical phenomena more thoroughly than categories-based measures; and presents adequate psychometric properties for assessing attachment in clinical and research contexts with adolescents and adults, both in its original or reduced versions ${ }^{32}$. Initially, the instrument was developed in France by Blaise Pierrehumbert' research team ${ }^{31}$ and has been translated into several languages (for further information, you can visit the author' web page: https://www.sites.google.com/ site/bpierreh/home). A 32-item abbreviated version has been developed and validated for the Spanish population (CAMIR-R) ${ }^{32}$.

This work is, from our knowledge, the first study using the CAMIR to assess attachment dimensions in persons with schizophrenia-spectrum disorders. The initial objective was to differentiate attachment styles by comparing a group of individuals with psychosis with a non-clinical sample using the reduced Spanish version (CAMIR-R). First, we hypothesised that CAMIR-R would result in a suitable instrument to assess attachment dimensions in individuals with psychosis and differentiate attachment profiles between both groups, with more insecure attachment in patients. Additionally, we report exploratory analyses relating attachment, psychotic symptoms and Social Functioning (SF), hypothesising that symptoms and attachment dimensions would predict SF areas within the clinical group. Finally, we conducted several mediation analyses to verify whether psychotic symptoms mediate the influence of attachment dimensions on different areas of SF. We hypothesised that attachment dimensions are directly linked to SF in persons with schizophreniaspectrum disorders, regardless of symptom severity. 


\section{Method}

\section{Participants and procedure}

A total of Seventy-nine adults participated voluntarily in the study, completing the self-administered CAMIR-R questionnaire. The clinical group involved forty-three individuals diagnosed with a psychotic disorder, according to the Diagnostic and Statistical Manual of Mental Disorders, fifth edition ${ }^{33}$. Patients were recruited via public mental health centres from Castellon and Valencia (Spain). The inclusion criteria were: 1) diagnosis of schizophrenia, schizoaffective or other psychotic disorder; 2) aged 18-65; 3) clinical stability; 4) medicationstabilised condition; 5) no history of drug abuse in the last four weeks; 6) complete assessment measures, and 7) native Spanish language. Twenty-four patients (55.8\%) had a diagnosis of schizophrenia; 5 (11.6\%) of schizoaffective disorder, and 14 (32.6\%) were non-specified psychosis. Fourteen (32.6\%) were outpatients; 19 (51.4\%) were attended in a day rehabilitation centre, and 10 (27\%) were inpatients. Ten (23.3\%) had spent less than five years since the onset of the disease; 10 (23.3\%) between 5 and 10 years and 23 (53.5\%) for more than ten years. The non-clinical group involved thirty-six individuals with no history of mental health problems, recruited as a convenience sample (age and gender) in the same geographic region. The study is part of a doctoral thesis approved by the Jaume I University Deontological Committee and complied with the Declaration of Helsinki ethical standards. The aim and conditions of the study were verbal and written described to prospective participants, and all of them provided written informed consent.

\section{Measures}

We used the short version of CAMIR to assess attachment styles and dimensions ${ }^{32}$. The CAMIR-R is a self-report questionnaire aimed at measuring attachment cognitions. It is based on subjects' evaluations of past and present attachment experiences and family functioning. It consists of 32 items rated on a 
five-point Likert-type scale. The CAMIR-R evaluates seven factors: 1) Security: Availability and support of the attachment figures, 2) Family concern, 3) Parental interference, 4) Value of parental authority, 5) Parental permissiveness, 6) Selfsufficiency and resentment against parents, and 7) Child traumatism. These factors are related to three attachment styles: secure, preoccupied or anxious, and dismissing or avoidant, then it does not evaluate disorganized (fearful) style because it may involve lapses in monitoring of logical thinking or discourse ${ }^{28}$. Internal consistency indexes ranged between 0.60 and 0.85 . CAMIR-R dimensions provide a valid and reliable assessment of attachment representations and the family functioning conception ${ }^{32}$.

Symptom severity was assessed using the Positive and Negative Syndrome Scale (PANSS) ${ }^{34,35}$. It is a semi-structured interview, comprising 30 items, which measure positive and negative symptoms and general psychopathology. Summing subscale scores can derive a total symptom score, and this was used as a measure of severity of psychiatric symptoms. The measure has shown to be reliable with Cronbach' alpha coefficients of 0.73 for the Positive scale, 0.83 for the Negative scale and 0.79 for the General Psychopathology scale ${ }^{34}$.

We assessed Social Functioning utilizing the short Spanish version of the Social Functioning Scale (SFS-R) ${ }^{36}$. SFS ${ }^{37}$ was designed to assess social functioning essential in schizophrenic patients through a medical interview. The original' 78 items were reduced to 13 items and then to 15 since two items related to the employment capacity were added. The SFS-R includes five areas: 1) social isolation, 2) interpersonal communication, 3) independence-execution, 4) independence-competence, and 5) employment-occupation, and allows a total score. The short form had a Cronbach' alpha of 0.76 and proved to be reliable and valid for use in clinical practice. In the present study, we excluded employmentrelated items and preferred to use more objective measures (current occupational status). 


\section{Data analyses}

Statistical analyses were performed using IBM SPSS version 23.0. Differences between the means of the two groups were evaluated through Student's $t$-test for two independent samples and Cohen's $d$ to calculate the effect sizes. We used non-parametrical $\chi^{2}$ to compare nominal variables, and Kolmogorov-Smirnov's $z$ to check whether variables followed a normal distribution.

Given the absence of previous studies using CAMIR-R in samples of individuals with psychosis, we calculated internal consistency indexes (Cronbach' alpha coefficients) to test the adequacy of the instrument within the clinical sample.

To determinate attachment styles, Lacasa and Muela's strategy was followed ${ }^{28}$ : dimension $1 T$ score $\geq 50$ in CAMIR-R was considered as suggestive of "secure attachment", and $T$ scores < 50 as "insecure attachment". The insecure style was divided into "preoccupied" (dimensions 2 and $3>$ dimension 6), or "avoidant" (dimension 6 > dimensions 2 and 3). Figure 1 shows the strategy.

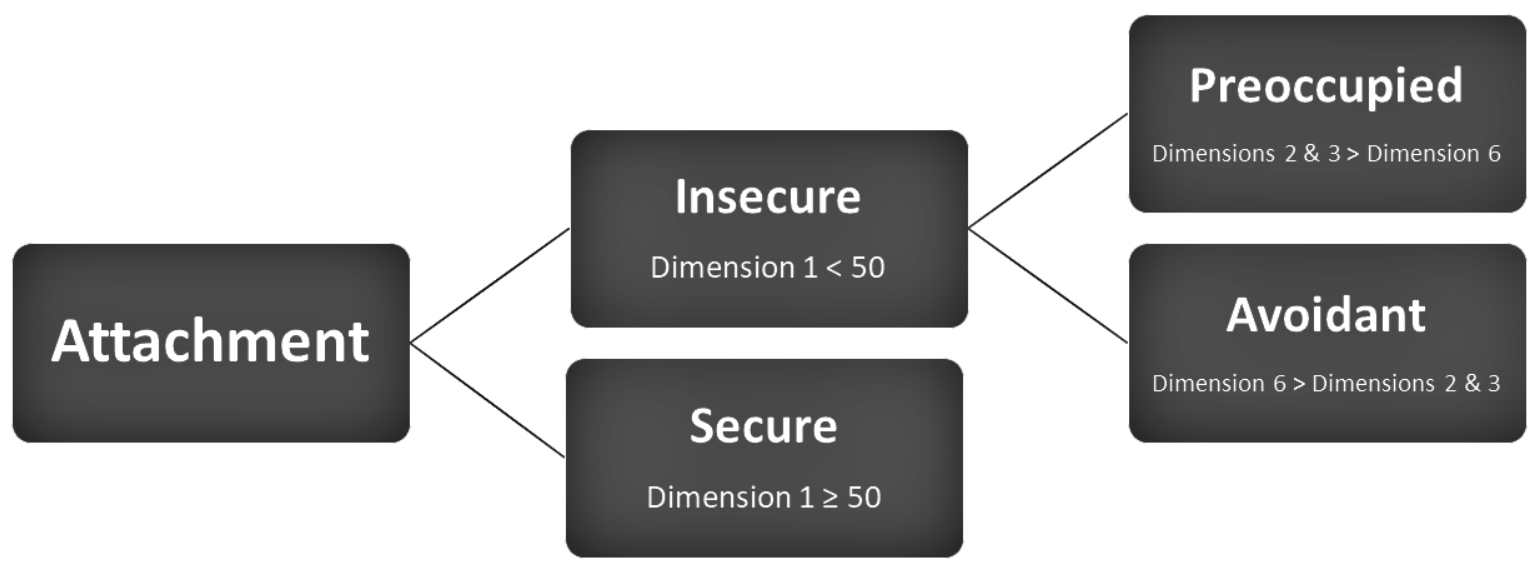

Primary strategy

Secondary strategy

Figure 1. Attachment styles: Primary and secondary strategies. 
Bivariate correlations were used to observe the relationships among variables. Multiple linear regressions with a stepwise method were performed to check possible predictors of SF areas. The criterion was: the probability of $F$ to enter $\leq 0.05$; the probability of $F$ to eliminate $\geq 0.10$. Age and gender were forced to enter in the first step as possible confounding variables. In the second step, PANSS scores were introduced. In the final step, CAMIR-R dimensions were introduced.

Additionally, we conducted a Mediation Analysis using JASP ${ }^{38}$ software version 0.11.1 to test total, direct and indirect effects of attachment dimensions to SF through symptom severity. Rosseel ${ }^{39}$ describes the mediation analysis in JASP. Attachment dimensions were introduced as predictors. Symptom severity (PANSS total score) was introduced as the mediator variable. SF areas were introduced in each model as the target output (predicted variable). Command options to calculate estimates were turned into standardized estimates, and missing values were handled as Full Information Maximum Likelihood. Testing whether an indirect effect exists, the bootstrap method (1000 replications) was applied. The confidence intervals were then computed using the bias-corrected percentile method, as suggested by Biesanz et al ${ }^{40}$. (see FOOT NOTE ${ }^{5}$ ).

\section{Results}

Table 1 shows the demographic and clinical characteristics of the participants. The clinical group was drawn mainly up of men, but a gender difference was not significant between the groups. No differences in age were found. Differences in all SF areas were found with large effect sizes. All CAMIR-R' dimensions follow a normal distribution (except parental interference) within the clinical group.

\footnotetext{
${ }^{5}$ Child traumatism was removed from the mediation analyses because collinearity problems prevent to run the bootstrap method.
} 
Table 1. Demographic and clinical characteristics of the sample

\begin{tabular}{|c|c|c|c|c|}
\hline & & Clinical & Control & $t / \chi^{2}$ \\
\hline \multicolumn{2}{|l|}{$\operatorname{Age}^{1}$} & 36.33 (9.28) & 36.19 (10.79) & 0.06 \\
\hline \multirow[t]{2}{*}{ Gender $^{2}$} & Male & 33 (76.7) & $22(61.1)$ & 2.26 \\
\hline & Female & $10(23.3)$ & $14(38.9)$ & \\
\hline \multirow[t]{3}{*}{ Education $^{2}$} & Elementary & $12(27.9)$ & $5(13.9)$ & $8.79 *$ \\
\hline & Middle & $21(48.8)$ & $11(30.6)$ & \\
\hline & University & 10 (23.3) & $20(55.6)$ & \\
\hline \multirow[t]{3}{*}{ Marital status $^{2}$} & Single & 30 (69.8) & $11(30.6)$ & $12.52^{* *}$ \\
\hline & Partner & $11(25.6)$ & $23(63.9)$ & \\
\hline & Separated & $2(4.7)$ & $2(5.6)$ & \\
\hline \multirow[t]{3}{*}{ Employment $^{2}$} & Active/student & $10(23.3)$ & $29(80.6)$ & $26.911^{* * *}$ \\
\hline & Unemployed & $14(32.6)$ & 5 (13.9) & \\
\hline & Pensioner & $19(43.2)$ & $2(5.6)$ & \\
\hline \multicolumn{2}{|l|}{ SFS Total ${ }^{1}$} & $21.95(4.64)$ & $32.14(1.46)$ & $-13.62^{* * *}$ \\
\hline \multicolumn{2}{|l|}{ Social Isolation } & $3.53(1.10)$ & $6.00(0.00)$ & $-14.71^{* * *}$ \\
\hline \multicolumn{2}{|c|}{ Interpersonal communication } & $5.63(2.51)$ & $9.64(1.07)$ & $-9.59 * * *$ \\
\hline \multicolumn{2}{|c|}{ Independence-execution } & $6.63(1.98)$ & $8.97(0.17)$ & $-7.75^{* * *}$ \\
\hline \multicolumn{2}{|c|}{ Independence-competence } & $6.16(1.53)$ & $7.53(0.74)$ & $-7.75^{* * *}$ \\
\hline \multicolumn{2}{|l|}{ PANSS Positive 1} & $14.02(5.66)$ & & \\
\hline \multicolumn{2}{|l|}{ PANSS Negative } & $15.37(5.00)$ & & \\
\hline \multicolumn{2}{|c|}{ PANSS General Psychopathology } & $29.21(7.93)$ & & \\
\hline \multicolumn{2}{|l|}{ TOTAL PANSS } & $58.37(16.19)$ & & \\
\hline
\end{tabular}

1 Mean (Standard deviation). ${ }^{2}$ Number (\%).

$* p<0.05$; $^{* *} p<0.01$; *** $p<0.001$

\section{CAMIR-R' internal consistency indexes}

Cronbach' alpha coefficients were calculated to test the adequacy of CAMIR-R. The results indicated that the instrument is reliable for use in patients with psychosis: Secure attachment $(a=0.90)$; Family concern $(a=0.76)$; Parental interference ( $a=0.78)$; Parental permissiveness ( $a=0.63$ ); Self-sufficiency ( $a=$ 0.64 ) and Childhood trauma $(a=0.81)$. Value of parental authority was not adequate $(a=0.45)$, so it was not used in the analyses. Alpha coefficients from 0.63 to 0.90 were considered appropriate for scales of less than eight items. 
Differences in attachment styles and dimensions

Tables 2 show the comparisons between groups. The clinical group obtained higher mean on insecure attachment with more avoidant than preoccupied style. Student's $t$-test indicated significant differences between groups in all the CAMIR-R dimensions, except parental permissiveness, with moderate to large effect sizes (Cohen's $d$ ).

Table 2a. Comparison between groups in attachment styles

\begin{tabular}{cll}
\hline & Clinical $^{1}$ & Control \\
\hline Attachment style & $\mathrm{N}(\%)$ & $\mathrm{N}(\%)$ \\
\hline Secure attachment & $11(26.2)$ & $24(66.7)$ \\
Insecure attachment & $31(73.8)$ & $12(33.3)$ \\
Preoccupied & $13(31.0)$ & $2(5.6)$ \\
Avoidant & $18(4.29)$ & $10(27.8)$ \\
\hline
\end{tabular}

${ }^{1}$ One subject lost by the system

Table $2 \mathrm{~b}$. Differences between groups in CAMIR-R' attachment dimensions

\begin{tabular}{lllll}
\hline & Clinical & Control & $\boldsymbol{t}$ & Cohen $\boldsymbol{d}$ \\
\hline Dimensions & Mean $(S D)$ & Mean $(S D)$ & & \\
\hline Secure & $41.12(15.24)$ & $52.11(5.86)$ & $-4.32^{* *}$ & 0.95 \\
Family concern & $49.38(12.11)$ & $37.20(10.49)$ & $4.71^{* *}$ & 1.08 \\
Interference & $56.11(13.18)$ & $46.65(9.88)$ & $3.62^{* *}$ & 0.31 \\
Permissiveness & $61.97(15.18)$ & $56.96(10.28)$ & 1.73 & 0.39 \\
Self-sufficiency & $55.29(10.91)$ & $48.64(7.83)$ & $3.12^{*}$ & 0.70 \\
Child traumatism & $67.17(15.80)$ & $47.87(6.14)$ & $7.30^{*}$ & 1.61 \\
\hline
\end{tabular}

$* p<0.01 ; * * p<0.001$

\section{Bivariate correlations}

No correlation between CAMIR-R dimensions and PANSS scales were found within the clinical group, except for self-sufficiency to general psychopathology subscale $(r=0.25 ; p<0.05)$. Gender (male) correlated with the PANSS negative $(r=0.29 ; p<0.05)$ and PANSS general psychopathology $(r=0.30$; 
$p<0.05)$. Years of evolution since the onset of the disease correlated with the employment status ( $r=0.72 ; p<.0001)$, family concern $(r=0.46 ; p<0.01)$ and self-sufficiency $(r=0.39 ; p<0.01)$. (see FOOT NOTE $\left.{ }^{6}\right)$.

Table 3 shows the bivariate correlations between CAMIR-R and SFS-R $(n=$ 79).

Table 4 shows the correlation matrix $(n=43)$.

Table 3. Bivariate correlations between CAMIR-R ${ }^{1}$ and SFS- $R^{2}(n=79)$

\begin{tabular}{lllllll}
\hline & Secure & $\begin{array}{l}\text { Fam. } \\
\text { Concern }\end{array}$ & Interference & Permissive & Self-surf & Trauma \\
\hline Total & $0.36^{* *}$ & $-0.47^{* * *}$ & $-0.22^{* *}$ & $-0.20^{*}$ & $-0.27^{* *}$ & $-0.48^{* * *}$ \\
Isolation & $0.34^{* *}$ & $-0.53^{* * *}$ & $-0.32^{* *}$ & $-0.20^{*}$ & $-0.27^{* *}$ & $-0.53^{* * *}$ \\
Communic. & $0.33^{* *}$ & $-0.42^{* * *}$ & -0.18 & -0.10 & $-0.27^{* *}$ & $-0.44^{* * *}$ \\
Indep-exec. & $0.22^{*}$ & $-0.29^{* *}$ & -0.13 & -0.15 & $-0.21^{*}$ & $-0.32^{* *}$ \\
Indep-com. & $0.25^{*}$ & $-0.27^{* *}$ & -0.14 & $-0.26^{*}$ & -0.06 & $-0.27^{* *}$ \\
\hline
\end{tabular}

${ }^{*} p<0.05 ;{ }^{* *} p<0.01 ;{ }^{* * *} p<0.001$ (2-tailed)

${ }^{1}$ CAMIR-R: Secure attachment; Familiy concern; Parental interference; Parental permissiveness; Selfsufficiency; Child traumatism.

${ }^{2}$ SFS-R: Social functioning (Total score); Social Isolation; Interpersonal communication; Independenceexecution; Independence-competence.

\section{Predictors of social functioning}

PANSS general psychopathology and parental interference conformed the best model $[F(4,38)=4.18 ; p=0.007]$ to predict general SF. Family concern predicted more social isolation $[F(3,39)=2.95 ; p<0.05]$. Negative symptoms predicted worse interpersonal communication $[F(3,39)=2.52 ; p=0.07]$ and, added to parental permissiveness, predicted a worse independence-competence $[F(4,38)=4.86 ; p=0.003]$. Educational level was inversely predicted by parental permissiveness and child traumatism $[F(4,38)=4.30 ; p=0.006]$. Age and family

\footnotetext{
6 The employment situation was considered as a nominal variable (1. active; 2. unemployed; 3 . pensioner) but it would be understood as a continuum where condition 1 is the most satisfactory, and condition 3 is the least satisfactory. Therefore, positive relations mean a worse situation.
} 
concern inversely predicted the employment status $[F(4,38)=10.82 ; p<.0001]$. Table 5 summarizes the regression models.

Table 4. Correlation Matrix $(n=43)$

\begin{tabular}{|c|c|c|c|c|c|c|c|c|c|c|c|c|c|c|c|c|c|c|}
\hline & 1 & 2 & 3 & 4 & 5 & 6 & 7 & 8 & 9 & 10 & 11 & 12 & 13 & 14 & 15 & 16 & 17 & 18 \\
\hline \multicolumn{19}{|l|}{ General SF } \\
\hline Isolation & $0.79^{* * *}$ & & & & & & & & & & & & & & & & & \\
\hline Communic & $0.71^{* * *}$ & $0.71^{* * *}$ & & & & & & & & & & & & & & & & \\
\hline Indep-exec & $0.46^{* *}$ & 0.07 & -0.19 & & & & & & & & & & & & & & & \\
\hline Indep-comp & $0.71^{* * *}$ & $0.42^{*}$ & $0.26^{*}$ & $0.34^{* *}$ & & & & & & & & & & & & & & \\
\hline Education & 0.01 & 0.06 & -0.05 & -0.01 & 0.09 & & & & & & & & & & & & & \\
\hline Employ & $-0.27^{*}$ & $-0.29^{*}$ & -0.10 & -0.18 & -0.22 & $-0.27^{*}$ & & & & & & & & & & & & \\
\hline Years onset & -0.16 & -0.13 & -0.06 & -0.10 & -0.17 & 0.06 & $0.72^{* * *}$ & & & & & & & & & & & \\
\hline PANSS P & $-0.37^{* *}$ & -0.24 & $-0.29^{*}$ & -0.19 & -0.24 & 0.21 & 0.15 & -0.00 & & & & & & & & & & \\
\hline PANSS N & $-0.39^{* *}$ & -0.19 & $-0.27^{*}$ & -0.09 & $-0.49^{* * *}$ & -0.12 & 0.09 & 0.20 & 0.25 & & & & & & & & & \\
\hline PANSS G & $-0.46^{* *}$ & -0.19 & -0.22 & $-0.33^{*}$ & $-0.47^{* * *}$ & -0.02 & 0.14 & 0.07 & $0.69^{* * *}$ & $0.55^{* * *}$ & & & & & & & & \\
\hline PANSS T & $-0.51^{* * *}$ & -0.26 & $-0.31^{*}$ & $-0.27^{*}$ & $-0.51^{* * *}$ & 0.02 & 0.17 & 0.12 & $0.79^{* * *}$ & $0.71^{* * *}$ & $0.93^{* * *}$ & & & & & & & \\
\hline CAMIR 1 & 0.00 & -0.03 & 0.03 & -0.06 & 0.08 & 0.08 & -0.04 & -0.04 & 0.09 & 0.01 & 0.04 & 0.09 & & & & & & \\
\hline CAMIR 2 & -0.18 & $-0.34^{*}$ & -0.14 & 0.03 & -0.10 & -0.19 & $0.58^{* * *}$ & $0.51^{* * *}$ & 0.13 & 0.17 & 0.14 & 0.19 & 0.18 & & & & & \\
\hline CAMIR 3 & 0.21 & 0.00 & 0.21 & 0.20 & 0.05 & -0.15 & $0.29^{*}$ & 0.19 & -0.01 & 0.03 & 0.12 & 0.06 & -0.04 & $0.40^{* *}$ & & & & \\
\hline CAMIR 5 & -0.08 & -0.09 & 0.08 & -0.06 & -0.22 & $-0.43^{* *}$ & 0.06 & -0.17 & 0.04 & -0.14 & 0.19 & 0.07 & 0.17 & -0.03 & 0.08 & & & \\
\hline CAMIR 6 & -0.06 & -0.03 & -0.13 & -0.04 & 0.10 & -0.13 & 0.21 & $0.30^{*}$ & 0.16 & 0.10 & $0.27^{*}$ & 0.22 & $-0.34^{*}$ & $0.33^{*}$ & $0.35^{*}$ & -0.00 & & \\
\hline CAMIR 7 & 0.10 & 0.01 & 0.05 & 0.12 & 0.07 & $-0.31^{*}$ & 0.17 & 0.03 & -0.16 & -0.19 & -0.10 & -0.17 & $-0.72^{* * *}$ & 0.17 & $0.35^{*}$ & 0.02 & $0.45^{* *}$ & \\
\hline
\end{tabular}

\section{Mediation analyses}

Table 6 shows the bootstrapping analyses of direct, indirect and total effects from attachment dimensions to SF via symptom severity. Family concern and parental interference were significantly linked to general SF. After testing whether indirect effects exist, total effects remained significant. Figure 2 shows the model with paths from attachment dimensions to general SF via symptom severity as mediator. The model explained $38 \%$ of the variance in general SF.

Mediation analyses for each specific SF area showed that family concern was linked to social isolation and, after testing whether indirect effects exist, total effects remained significant $(\beta=-0.46 ; p<0.01)$. Parental interference was linked to interpersonal communication $(\beta=0.34 ; p<0.05)$. Secure attachment and parental permissiveness directly linked to independence-competence, but their 
effects were mediated by symptom severity. Nevertheless, total effects for parental permissiveness remained close to significant levels $(\beta=-0.28 ; p=0.06)$. Parental permissiveness was linked to educational level $(\beta=-0.47 ; p<0.001)$, and family concern was strongly linked to employment status, regardless of symptoms $(\beta=0.61 ; p<0.001)$. Figure 3 shows plots with models describing links between attachment, symptom severity and specific areas of SF.

Table 5. Summary of the linear regression models

\begin{tabular}{|c|c|c|c|c|c|c|}
\hline \multicolumn{7}{|c|}{ Predicted variable: General social functioning } \\
\hline Model & Predictor & $B$ & $\beta$ & $R^{2}$ & Change in $R^{2}$ & $p$-value \\
\hline \multirow[t]{2}{*}{3} & Psychopathology & -0.31 & -0.53 & 0.23 & 0.23 & 0.001 \\
\hline & Parental interference & 0.10 & 0.28 & 0.3 & 0.08 & 0.050 \\
\hline \multicolumn{7}{|c|}{ Predicted variable: Social isolation } \\
\hline Model & Predictor & $B$ & $\beta$ & $R^{2}$ & Change in $R^{2}$ & $p$-value \\
\hline 2 & Family concern & -0.04 & -0.40 & 0.19 & 0.14 & 0.015 \\
\hline \multicolumn{7}{|c|}{ Predicted variable: Interpersonal communication } \\
\hline Model & Predictor & $B$ & $\beta$ & $R^{2}$ & Change in $R^{2}$ & p-value \\
\hline 2 & PANSS negative & -0.17 & -0.36 & 0.16 & 0.12 & 0.023 \\
\hline \multicolumn{7}{|c|}{ Predicted variable: Independence-competence } \\
\hline Model & Predictor & $B$ & $\beta$ & $R^{2}$ & Change in $R^{2}$ & $p$-value \\
\hline \multirow[t]{3}{*}{3} & PANSS negative & -0.15 & -0.53 & 0.25 & 0.22 & $<.000$ \\
\hline & Parental & & & 0.34 & & \\
\hline & Permissiveness & -0.13 & -0.32 & & 0.9 & 0.027 \\
\hline
\end{tabular}

Predicted variable: Educational level

\begin{tabular}{|c|c|c|c|c|c|c|}
\hline Model & Predictor & $B$ & $\beta$ & $R^{2}$ & Change in $R^{2}$ & $p$-value \\
\hline \multirow[t]{3}{*}{3} & Parental & & & 0.22 & & \\
\hline & Permissiveness & -0.02 & -0.42 & 0.31 & 0.17 & 0.004 \\
\hline & Child traumatism & -0.01 & -0.31 & & 0.09 & 0.029 \\
\hline
\end{tabular}

\begin{tabular}{|c|c|c|c|c|c|c|}
\hline \multicolumn{7}{|c|}{ Predicted variable: Employment status } \\
\hline Model & Predictor & $B$ & $\beta$ & $R^{2}$ & Change in $R^{2}$ & $p$-value \\
\hline \multirow[t]{2}{*}{3} & Age & 0.04 & 0.40 & 0.31 & 0.31 & 0.003 \\
\hline & Family concern & 0.03 & 0.41 & 0.45 & 0.15 & 0.002 \\
\hline
\end{tabular}

$B$ : non standardised coefficients. $\beta$ : standardised coefficients. All models included contributions of gender and age. 
Direct effects

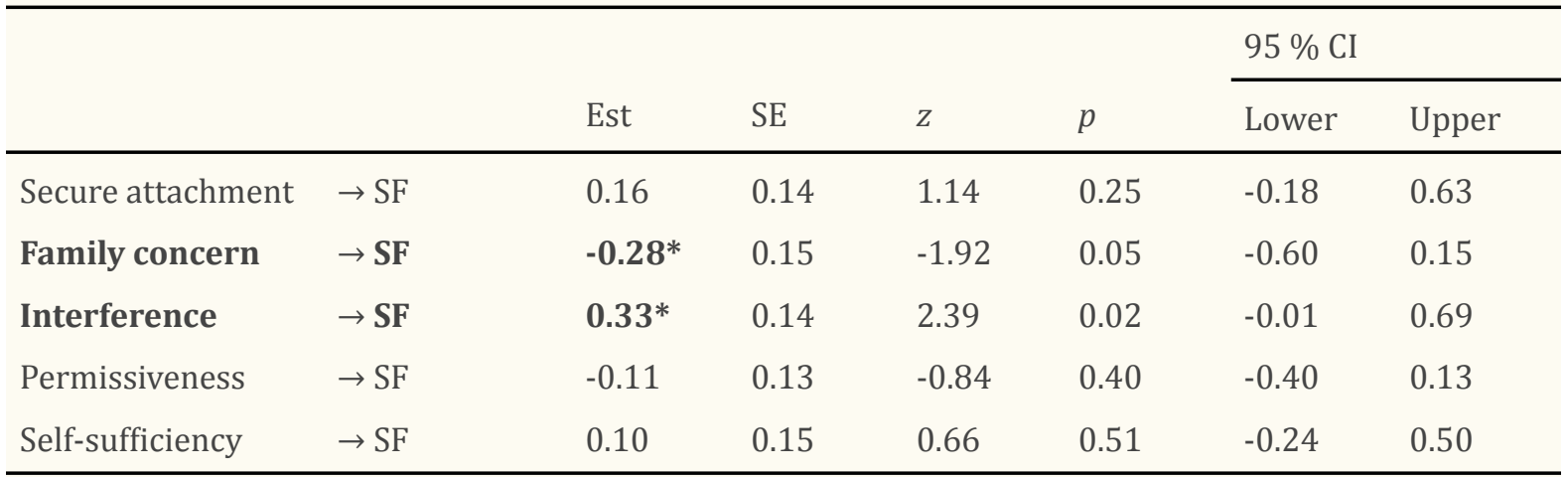

Indirect effects

\begin{tabular}{llllllll}
\hline & & & & & \multicolumn{2}{c}{$95 \%$ CI } \\
\cline { 5 - 8 } & & Est & SE & $z$ & $p$ & Lower & Upper \\
\hline Secure attachment & $\rightarrow$ PANSS $\rightarrow$ SF & -0.06 & 0.09 & -0.70 & 0.49 & -0.22 & 0.16 \\
Family concern & $\rightarrow$ PANSS $\rightarrow$ SF & -0.06 & 0.09 & -0.69 & 0.49 & -0.34 & 0.12 \\
Interference & $\rightarrow$ PANSS $\rightarrow$ SF & 0.04 & 0.09 & 0.41 & 0.68 & -0.13 & 0.25 \\
Permissiveness & $\rightarrow$ PANSS $\rightarrow$ SF & -0.03 & 0.08 & -0.39 & 0.70 & -0.20 & 0.14 \\
Self-sufficiency & $\rightarrow$ PANSS $\rightarrow$ SF & -0.11 & 0.09 & -1.17 & 0.24 & -0.35 & 0.12 \\
\hline
\end{tabular}

\section{Total effects}

\begin{tabular}{llllllll}
\hline & & & & & & \multicolumn{2}{c}{$95 \%$ CI } \\
\cline { 5 - 7 } & & Est & SE & $z$ & $p$ & Lower & Upper \\
\hline Secure attachment & $\rightarrow$ SF & 0.10 & 0.17 & 0.61 & 0.54 & -0.24 & 0.71 \\
Family concern & $\rightarrow$ SF & $-\mathbf{0 . 3 4 *}$ & 0.17 & -2.00 & 0.05 & -0.67 & 0.13 \\
Interference & $\rightarrow$ SF & $\mathbf{0 . 3 7 ^ { * }}$ & 0.16 & 2.25 & 0.03 & -0.06 & 0.78 \\
Permissiveness & $\rightarrow$ SF & -0.14 & 0.15 & -0.92 & 0.36 & -0.44 & 0.13 \\
Self-sufficiency & $\rightarrow$ SF & -0.01 & 0.17 & -0.06 & 0.95 & -0.38 & 0.46 \\
\hline
\end{tabular}

Delta method standard errors, bias-corrected percentile bootstrap confidence intervals.

PANSS: PANSS Total score (symptom severity). SF: General social functioning. Est: Estimates (standardised coefficients). SE: Standard error. CI: Confidence interval. Bold characters mean significant effects.

$* p<0.05$. 


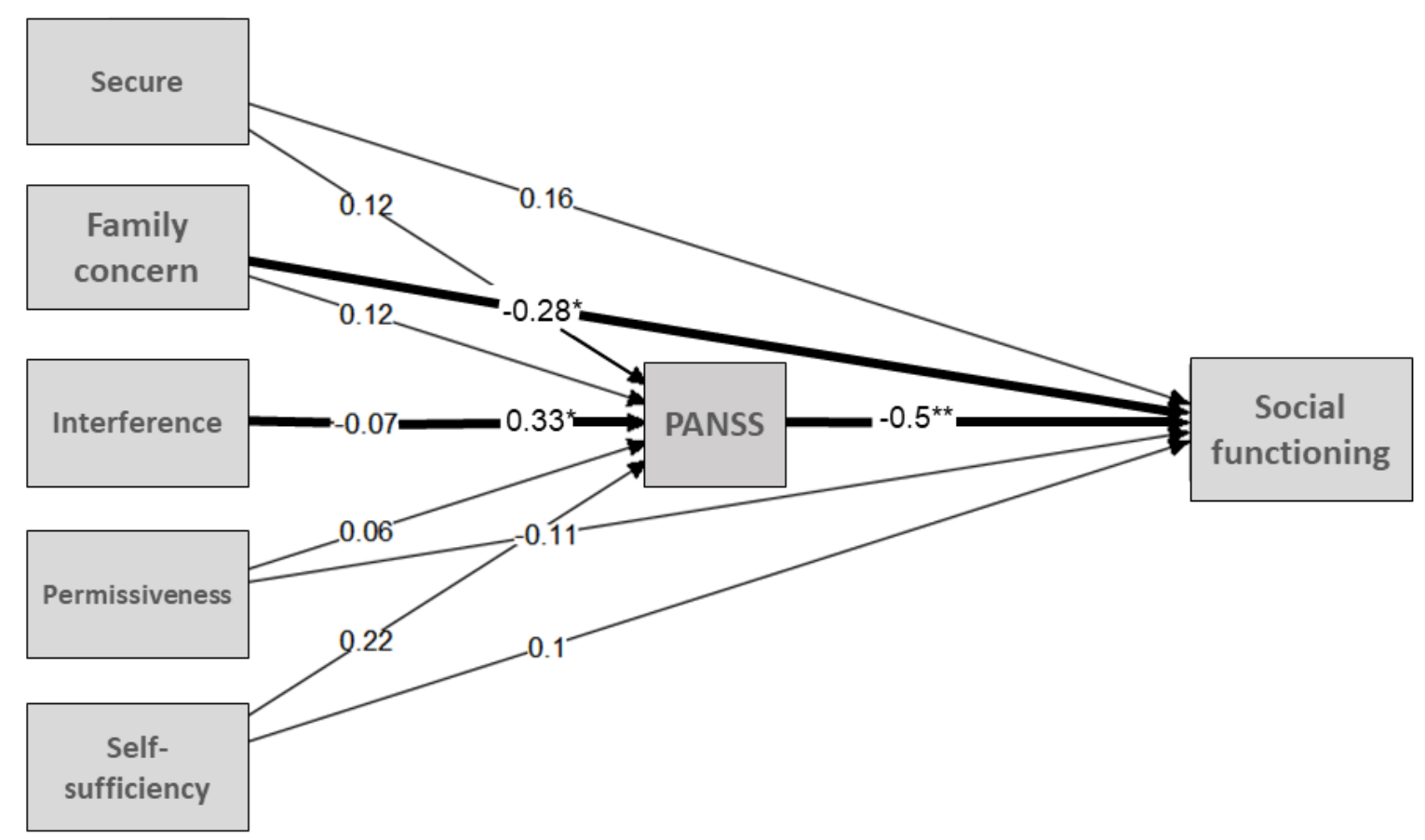

Figure 2. Model with paths from attachment dimensions to General Social Functioning via symptom severity as mediator.

Attachment dimensions (family concern and parental interference) are linked to SF, regardless of symptoms. The model explains $38 \%$ of the variance in general SF.

${ }^{*} p<0.05 ;{ }^{* *} p<0.01$ 


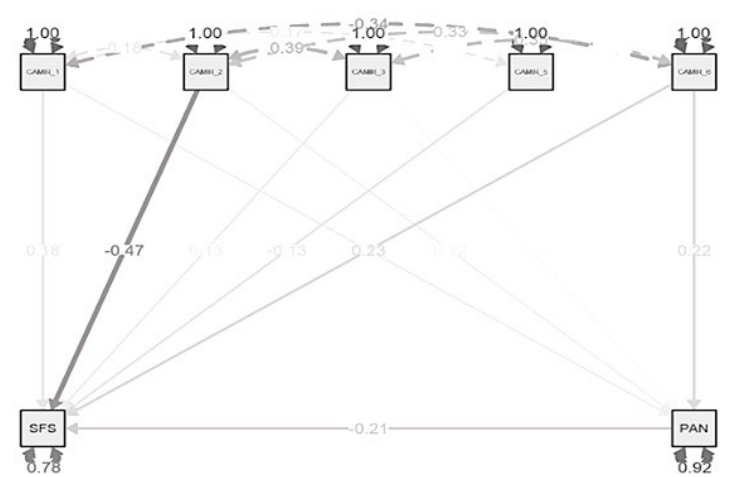

Model with paths from attachment dimensions to Social isolation. The model explains $23 \%$ of the variance in Social Isolation.

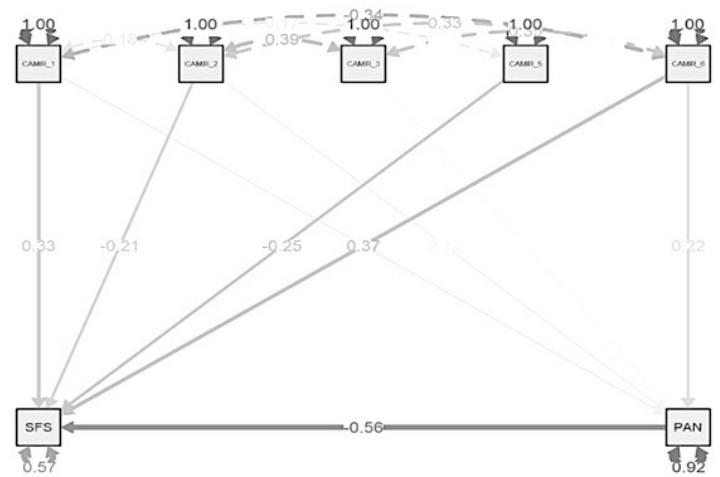

Model with paths from attachment dimensions to Independence-competence. The model explains $42 \%$ of the variance in Independencecompetence. NOTE: After testing whether indirect effects exist, no total effect remained significant from attachment dimensions to Independencecompetence.

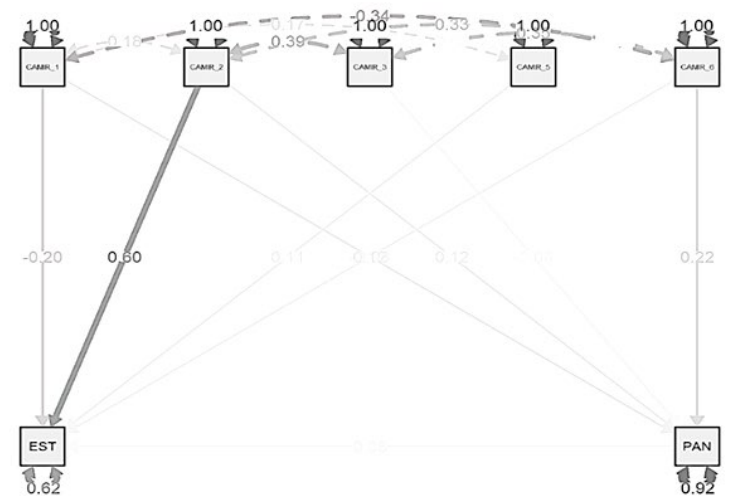

Model with paths from attachment dimensions to Employment status. The model explains 34\% of the variance in the Employment situation.

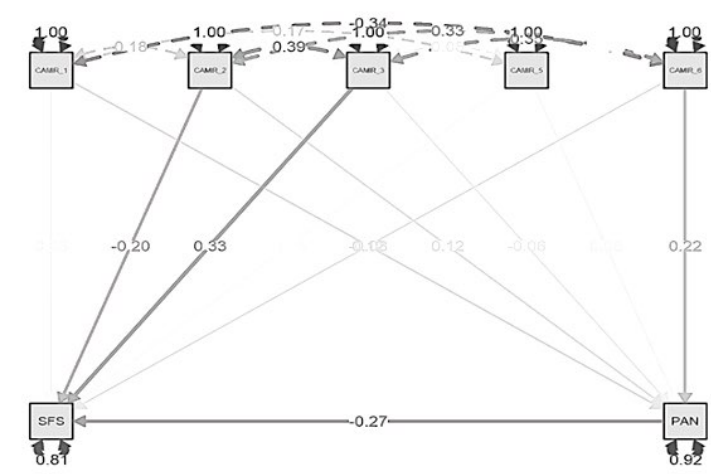

Model with paths from attachment dimensions to Interpersonal communication. The model explains $19 \%$ of the variance in Interpersonal communication.

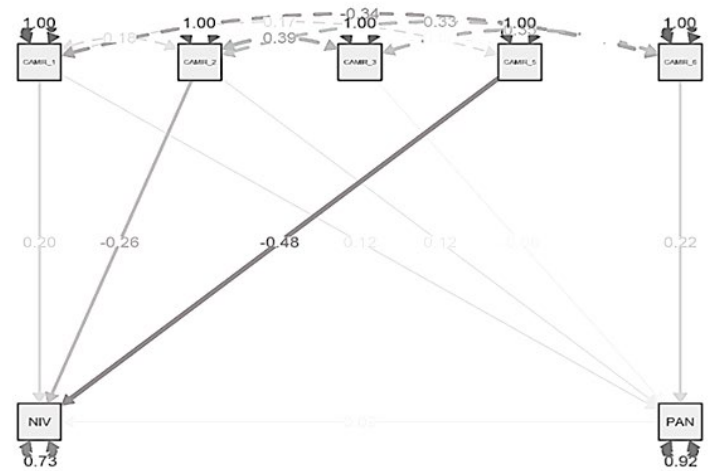

Model with paths from attachment dimensions to Educational level. The model explains $27 \%$ of the variance in Educational level.

NOTE: Continuous lines link independent variables (attachment dimensions) to the dependent variable or the mediator (PANSS total score), and from the mediator (PANSS total score) to the dependent variable. Discontinuous lines link independent variables among them.

Figure 3. Model plots with the Mediation analyses for each specific area of Social Functioning. 


\section{Discussion and Conclusions}

The instrument we focused on to assess attachment patterns (CAMIR-R) obtained good internal consistency coefficients and is considered reliable to use in samples of persons with schizophrenia-spectrum disorders. As expected, attachment differentiates between people with psychosis and healthy population, being more insecure in people with psychosis. Moreover, attachment dimensions predict several SF areas and do it regardless of psychotic symptoms. Differences between groups in all SF areas were found with large effect sizes, indicating relevant impairments in SF in persons with psychosis. As well, gender (male) predicts symptom severity.

The results show a greater insecure attachment in the clinical group with a higher percentage of avoidant style, according to recent reviews ${ }^{7,8,19}$. Negative symptoms of schizophrenia often present as "avoidant" types of behaviour and result in a lack of meaningful interpersonal relationships ${ }^{29}$. Moreover, higher scores in child traumatism differentiate people with psychosis from the healthy population, according to the high rates of attachment disrupting events that individuals with psychosis tend to experience2,20. Exposure to traumatic life experiences can significantly impact the pathogenesis of psychotic experiences as a precipitating or exacerbating factor and can lead to psychosis through many pathways that intersect with other genetic or environmental risk factors ${ }^{41}$. Having suffered traumatic experiences is related to the development of psychosis, but not necessary to symptom severity 42 . When we consider the complete sample, secure attachment positively correlates to all SF areas. Family concern (preoccupied attachment), self-sufficiency (avoidant attachment) and child traumatism relate to worse SF. How attachment style differences are expressed in real-time as individuals navigate their real-life settings has remained an area mostly untapped by research in the field ${ }^{43}$. Our results are consistent with recent findings demonstrating how predictions derived from attachment theory play a role in the natural flow of real-life ${ }^{43}$. Previous works demonstrate a significant positive association between preoccupied attachment and symptom severity ${ }^{44}$. Contrary to this previous literature, the preoccupied attachment does not 
correlate with positive or negative symptoms, but self-sufficiency (representing avoidant style) correlates to general psychopathology. As cited above, symptoms of schizophrenia often present as "avoidant" types of behaviour29.

General psychopathology predicts worse general SF, while parental interference predicts better outcomes in it. According to Balluerka et al. ${ }^{32}$, this dimension refers to the ambivalence with the attachment figures and is related to the preoccupied attachment style. Although people with a preoccupied attachment style may have maladaptive interpersonal patterns, their continued engagement in social relationships may act as a buffer against symptoms becoming clinically significant 45 and, due to the characteristics of the sample (most long-term stable patients), we might suppose that family relationships would be able to provide enough social support. Findings also demonstrate that persons who experienced preoccupied attachment tend to have higher levels of social isolation ${ }^{8}$. The family concern is associated with preoccupied attachment and is characterised by the perception of intense separation anxiety of loved ones and an excessive current concern for attachment figures. The person avoids moving away from them by hyperactivating his/her attachment system; this scheme aims to maintain proximity and ensure the accessibility of their attachment figures ${ }^{28}$.

Moreover, family concern predicts worse employment status and is related to the years since the onset of the disease that also has a negative impact on the employment situation. Thus, separation anxiety hinders personal autonomy. However, other studies find that most individuals with an avoidant attachment pattern also lack flexibility in relationships and are very isolated ${ }^{46}$. We find that negative symptoms predict worse interpersonal communication and lower levels of independence-competence in daily life, according to the previous studies ${ }^{29}$. Parental permissiveness is also related to less independence-competence and is the best predictor of a worse educational level. This dimension refers to the memories of having suffered from a lack of limits and insufficient parental guidance in childhood. This parental representation is typical of an insecure 
style ${ }^{28}$. Persons who remember having adequate limits during childhood achieved a better educational level.

Beyond the findings discussed above, the direction in which attachment dimensions influence SF is an important question to clarify. Some works have investigated whether insecure attachment and cognitive biases serve as mediators between traumatic life events and psychotic-like experiences in nonclinical samples ${ }^{16}$ and others, in line with the theory of an affective pathway to psychosis after exposure to childhood trauma, suggest multiple paths between trauma and psychosis using a network and pathway analyses ${ }^{47}$. Nevertheless, on that point is not enough research about the direct influence of attachment on SF. In the current study, we conducted mediation analyses to explore pathways from attachment dimensions to SF via symptom severity as mediator. According to our hypothesis, the analyses confirm that several attachment dimensions are linked to SF, regardless of symptoms. The overall model explains $38 \%$ of the variance in general SF. The detailed models for specific SF areas demonstrate how the family concern is linked to social isolation and employment status, regardless of symptoms. As well, parental interference is linked to the interpersonal communication and parental permissiveness to the educational level. Despite its potential role as a predictor of SF, attachment only accounts for a limited part in real-life functioning, and thus, more research is needed to clarify this issue. Previous findings indicate that the interplay between cognitive biases, selfdisturbances and insecure attachment may be of outstanding importance in the onset of psychotic experiences ${ }^{43}$.

\section{Main findings}

Social functioning is highly impaired in persons with schizophreniaspectrum disorders. As far as we know, this is the first study that uses CAMIR to assess attachment styles in people with psychotic disorders. The instrument demonstrated suitability for use in psychotic samples, showing acceptable internal consistency indexes. Moreover, our findings support our hypotheses: 
according to the literature, persons with psychosis show an insecure attachment pattern with higher scores in all dimensions and a higher prevalence of the avoidant style. Most previous studies focused on the relationships between attachment styles and psychotic symptomatology, but their results remain unclear. Our results suggest the idea that having suffered insecure attachment is related to the vulnerability to develop psychosis, but not necessary to symptom severity. General psychopathology, negative symptoms and some attachment dimensions predict a worse social functioning. Parental interference may act as a protective factor to keep a better social functioning, but separation anxiety hinders personal autonomy, explaining why patients with higher levels of family concern tend to have more social isolation and worse employment status. Finally, persons with psychosis who remember having adequate limits during childhood tend to be more competent and autonomous in their daily-life activities and achieve a better educational level.

Beyond that, our findings highlight a direct link between attachment and social functioning in schizophrenia-spectrum disorders. The methodology of the study, using mediation analyses, allows concluding that several attachment dimensions influence social functioning, regardless of symptom severity.

\section{Limitations and future research}

Our study presents some limitations. First, the small sample size does not allow us to generalize the results easily. Likewise, a significant portion of clinical subjects were long-term patients that were being treated in psychosocial rehabilitation services, and younger patients or those with early psychosis episodes would be underrepresented in the sample. Another limitation is the cross-sectional design that hinders the determination of causal relationships, since it is impossible to know whether the attachment experiences that patients remember preceded the disease or, on the contrary, the disease modified the attachment-related cognitions and memories. Nevertheless, previous studies 
have demonstrated that retrospective self-reports can be used to assess attachment disrupting events reliably ${ }^{42}$.

Future research should attempt to address the issue of how attachment relates to the development of psychosis and the interaction among attachment dimensions, basic and social cognitive domains or other environmental risk and protective factors. Attachment patterns account for a limited part of real-life functioning, and therefore, there is a need to investigate them more ecologically, using better measures, longitudinal designs, more appropriate samples and testing additional moderators to improve these limitations.

\section{Clinical implications}

If attachment dimensions predict social functioning in individuals with schizophrenia-spectrum disorders, attachment assessment would be required. Interventions in some patients aimed at strengthening attachment bonds and modifying interactional patterns may effectively prevent them from developing psychosis or improve psychosocial skills in real life 6 . It could be important for clinicians to identify patients' attachment styles. According to Strand et al. ${ }^{44}$, such knowledge would help professionals to understand better the persons' capacity to handle the distress and to address interventions aiming to target each patient's specific needs.

Acknowledgments: The authors thank Silvia Edo and Nuria Bort-Mora for the insight and support on this project.

\section{References}

1.Sheinbaum T, Bedoya E, Ros-Morente A, Kwapil TR, Barrantes-Vidal N. Association between attachment prototypes and schizotypy dimensions in two independent non-clinical samples of Spanish and American young adults. Psychiatry Res. 2013;210(2):408-13. http://doi.org/10.1016/j.psychres.2013.07.020 
2.Bailey T, Alvarez-Jimenez M, Garcia-Sanchez AM, Hulbert C, Barlow E, Bendall S. Childhood Trauma Is Associated With Severity of Hallucinations and Delusions in Psychotic Disorders: A Systematic Review and Meta-Analysis. Schizophr Bull. 2018;44(5):1111-22. https://doi.org/10.1093/schbul/sbx161

3.Bowlby J. The role of attachment in personality development. In: Bowlby J, editor. A Secure Base: Parent-Child Attachment and Healthy Human Development. London: Routledge; 1988. p. 119-36.

4.Bifulco A, Thomas G. Understanding adult attachment in family relationships: Research, assessment and intervention. London: Taylor and Francis; 2012.

5.Hardy A, Emsley R, Freeman D, Bebbington P, Garety PA, Kuipers EE, et al. Psychological Mechanisms Mediating Effects Between Trauma and Psychotic Symptoms: The Role of Affect Regulation, Intrusive Trauma Memory, Beliefs, and Depression. Schizophr Bull. 2016;42(suppl_1):S34-43. https://doi.org/10.1093/ schbul/sbv175

6.Rajkumar RP. Childhood attachment and schizophrenia: The "attachmentdevelopmental-cognitive" (ADC) hypothesis. Med Hypotheses. 2014;83(3):276-81. http://doi.org/10.1016/j.mehy.2014.05.017

7.Gumley AI, Taylor HEF, Schwannauer M, MacBeth A. A systematic review of attachment and psychosis: Measurement, construct validity and outcomes. Acta Psychiatr Scand. 2014;129:257-74. http://doi.org/10.1111/acps.12172

8.Korver-Nieberg N, Berry K, Meijer CJ, De Haan L. Adult attachment and psychotic phenomenology in clinical and non-clinical samples: A systematic review. Psychol Psychother Theory Res Pract. 2014;87(2):127-54. http://doi.org/10.1111/papt. 12010

9.Chatziioannidis S, Andreou C, Agorastos A, Kaprinis S, Malliaris Y, Garyfallos G, et al. The role of attachment anxiety in the relationship between childhood trauma and schizophrenia-spectrum psychosis. Psychiatry Res. 2019;276:223-31. http:// doi.org/10.1016/j.psychres.2019.05.021

10.Ponizovsky AM, Vitenberg E, Baumgarten-Katz I, Grinshpoon A. Attachment styles and affect regulation among outpatients with schizophrenia: Relationships to symptomatology and emotional distress. Psychol Psychother Theory Res Pract. 2013;86(2):164-82. http://doi.org/10.1111/j.2044-8341.2011.02054.x 
11.Wickham S, Sitko K, Bentall RP. Insecure attachment is associated with paranoia but not hallucinations in psychotic patients: The mediating role of negative selfesteem. Psychol Med. 2015;45(7):1495-507. http://doi.org/10.1017/ S0033291714002633

12.Berry K, Wearden A, Barrowclough C, Oakland L, Bradley J. An investigation of adult attachment and the nature of relationships with voices. $\mathrm{Br} J$ Clin Psychol. 2012;51(3):280-91. http://doi.org/10.1111/j.2044-8260.2011.02027.x

13.Donohoe G, Spoletini I, McGlade N, Behan C, Hayden J, O’Donoghue T, et al. Are relational style and neuropsychological performance predictors of social attributions in chronic schizophrenia? Psychiatry Res. 2008;161(1), 19-27. http:// doi.org/10.1016/j.psychres.2007.10.001

14.MacBeth A, Gumley A, Schwannauer M, Fisher R. Attachment states of mind, mentalization, and their correlates in a first-episode psychosis sample. Psychol Psychother Theory Res Pract. 2011;84(1):42-57. http://doi.org/doi: $10.1348 / 147608310 \times 530246$

15.Berry K, Barrowclough C, Wearden A. Attachment theory: A framework for understanding symptoms and interpersonal relationships in psychosis. Behav Res Ther. 2008;46(12):1275-82. http://doi.org/10.1016/j.brat.2008.08.009

16.Gawęda Ł, Pionke R, Krężołek M, Prochwicz K, Kłosowska J, Frydecka D, et al. Selfdisturbances, cognitive biases and insecure attachment as mechanisms of the relationship between traumatic life events and psychotic-like experiences in nonclinical adults - A path analysis. Psychiatry Res. 2018;259:571-8. http://doi.org/ 10.1016/j.psychres.2017.11.009

17.McGonagle G, Bucci S, Varese F, Raphael J, Berry K. Is adult attachment associated with engagement with services? A systematic literature review. J Ment Heal. 2019;1-12. Available from: https://doi.org/10.1080/09638237.2019.1608922

18. White R, Haddock G, Varese F. Supporting the intimate relationship needs of service users with psychosis: what are the barriers and facilitators? J Ment Heal [Internet]. 2019;e8928;1-7. https://doi.org/10.1080/09638237.2019.1608928

19.Carr SC, Hardy A, Fornells-Ambrojo M. Relationship between attachment style and symptom severity across the psychosis spectrum: A meta-analysis. Clin Psychol Rev. 2018;59:145-58. http://doi.org/10.1016/j.cpr.2017.12.001 
20.Rössler W, Ajdacic-Gross V, Rodgers S, Haker H, Müller M. Childhood trauma as a risk factor for the onset of subclinical psychotic experiences: Exploring the mediating effect of stress sensitivity in a cross-sectional epidemiological community study. Schizophr Res. 2016;172(1-3):46-53. http://doi.org/10.1016/j.schres. 2016.02.006

21. Rokita KI, Dauvermann MR, Donohoe G. Early life experiences and social cognition in major psychiatric disorders: A systematic review. Eur Psychiatry. 2018;53:123-133. https://doi.org/10.1016/j.eurpsy.2018.06.006

22.Debbané M, Salaminios G, Luyten P, Badoud D, Armando M, Solida Tozzi A, et al. Attachment, Neurobiology, and Mentalizing along the Psychosis Continuum. Front Hum Neurosci. 2016;10:406. https://doi.org/10.3389/fnhum.2016.00406

23.Pilton M, Bucci S, McManus J, Hayward M, Emsley R, Berry K. Does insecure attachment mediate the relationship between trauma and voice-hearing in psychosis? Psychiatry Res. 2016;246:776-82. http://doi.org/10.1016/j.psychres. 2016.10.050

24.Popovic D, Schmitt A, Kaurani L, Senner F, Papiol S, Malchow B, et al. Childhood Trauma in Schizophrenia: Current Findings and Research Perspectives. Front Neurosci. 2019;13:274. http://doi.org/10.3389/fnins.2019.00274

25.Sheinbaum T, Bifulco A, Ballespí S, Mitjavila M, Kwapil TR, Barrantes-Vidal N. Interview investigation of insecure attachment styles as mediators between poor childhood care and schizophrenia-spectrum phenomenology. PLoS One. 2015;10(8). http://doi.org/10.1371/journal.pone.0135150

26.Aydın 0, Balıkçı K, Taş C, Ünal-Aydın P, Taneli F, Esen-Danacı A. Assessing the relationship between attachment, parental attitude and plasma oxytocin in schizophrenia patients and their unaffected siblings. Nord J Psychiatry. 2019;73:51-57. https://doi.org/10.1080/08039488.2018.1554698

27.Speck LG, Schöner J, Bermpohl F, Heinz A, Gallinat J, Majić T, et al. Endogenous oxytocin response to film scenes of attachment and loss is pronounced in schizophrenia. Soc Cogn Affect Neurosci. 2019;14(1):109-17. https://doi.org/ $10.1093 /$ scan/nsy110

28.Lacasa F, Muela A. Guía para la aplicación e interpretación del cuestionario de apego CaMir-R. Psicopatol Salud Ment. 2014;24:83-93. 
29. Crowell JA. The adult attachment interview. In: Farnfield S, Holmes P, eds. The Routledge Handbook of Attachment: Assessment. London: Routledge; 2014. p. 144-55.

30.Molina P, Casonato M, Sala MN, Testa S. The Factor Structure of the CA-MIR as Evaluated Using Confirmatory Factor Analysis [Internet]. Front Psychol. 2018;9:190. https://doi.org/10.3389/fpsyg.2018.00190

31.Pierrehumbert B, Karmaniola A, Sieye A, Meister C, Miljkovitch R, et al. Les modeles de relations: Developpement d'un autoquestionnaire d'attachement pour adultes. Psychiatr l'Enfant. 1996;39(1):161-206.

32.Balluerka N, Lacasa F, Gorostiaga A, Muela A. CAMIR-R: Versión Reducida del cuestionario CAMIR. Psicothema [Internet]. 2011;23:486-94. Available from: http://www.psicothema.com/psicothema.asp?id=3913

33.American Psychiatric Association. Diagnostic and Statistical Manual of Mental Disorders: DSM-5. Washintong DC: American Psychiatric Association; 2014.

34.Kay SR, Fiszbein A, Opler LA. The positive and negative syndrome scale (PANSS) for schizophrenia. Schizophr Bull. 1987;13(2):261-76. https://doi.org/10.1093/ schbul/13.2.261

35.Peralta V, Cuesta MJ. Psychometric properties of the Positive and Negative Syndrome Scale (PANSS) in schizophrenia. Psychiatry Res. 1994;53(1):31-40. https:// doi.org/10.1016/0165-1781(94)90093-0

36.Alonso J, Olivares JM, Ciudad A, Manresa JM, Casado A, Gilaberte I. Desarrollo y validación de la versión corta de la Escala de Funcionamiento Social en esquizofrenia para su uso en la práctica clínica. Actas Esp Psiquiatr. 2008;36(2): 102-10.

37.Torres A, Olivares JM. Validation of the Spanish version of the social functioning scale. Actas Esp Psiquiatr. 2005;33(4):216-20.

38.JASP Team. JASP (Version 0.11.1) [Computer software]. 2019 [accessed 1 March 2020]. Available from: https://jasp-stats.org/

39. Rosseel Y. Lavaan: An R Package for Structural Equation Modeling. J Stat Software. 2012;48(2):1-36. http://dx.doi.org/10.18637/jss.v048.i02 
40.Biesanz JC, Falk CF, Savalei V. Assessing Mediational Models: Testing and Interval Estimation for Indirect Effects. Multivariate Behav Res. 2010;45(4):661-701. http://doi.org/10.1080/00273171.2010.498292

41.Gibson LE, Alloy LB, Ellman LM. Trauma and the psychosis spectrum: A review of symptom specificity and explanatory mechanisms. Clin Psychol Rev. 2016;49:92105. http://doi.org/10.1016/j.cpr.2016.08.003

42.Simpson S, Phillips L, Baksheev G, Garner B, Markulev C, Phassouliotis C, et al. Stability of retrospective self-reports of childhood trauma in first-episode psychosis. Early Interv Psychiatry. 2019;13(4):908-13. https://doi.org/10.1111/ eip. 12700

43.Sheinbaum T, Kwapil TR, Ballespí S, Mitjavila M, Chun CA, Silvia PJ, et al. Attachment style predicts affect, cognitive appraisals, and social functioning in daily life. Front Psychol. 2015;6:296. https://doi.org/10.3389/fpsyg.2015.00296

44.Strand J, Goulding A, Tidefors I. Attachment styles and symptoms in individuals with psychosis. Nord J Psychiatry. 2015;69(1):67-72. http://doi.org/ $10.3109 / 08039488.2014 .929740$

45.Blair MA, Nitzburg G, DeRosse P, Karlsgodt KH. Relationship between executive function, attachment style and psychotic like experiences in typically developing youth. Schizophr Res. 2018;197:428-33. http://doi.org/10.1016/j.schres. 2018.02.026

46.DeGangi GA. Addressing Attachment and Problems of Intimacy: How to Build Healthy Emotional Connections. In: DeGangi GA, ed. The Dysregulated Adult: Elsevier Inc; 2012. p. 381-28. https://doi.org/10.1016/B978-0-12-385011-9.00010-9

47.Isvoranu AM, van Borkulo CD, Boyette LL, Wigman JTW, Vinkers CH, Borsboom D, et al. A Network Approach to Psychosis: Pathways Between Childhood Trauma and Psychotic Symptoms. Schizophr Bull. 2016;43(1):187-96. https://doi.org/ $10.1093 / \mathrm{schbul} / \mathrm{sbw} 055$ 


\section{Capítulo 7 Estudio 3}

\section{La centralidad del apego seguro en una red interactiva entre los síntomas psicóticos, la cognición y las dimensiones del apego ${ }^{7}$}

\section{Resumen}

La investigación en el campo de la psicosis sugiere en término generales que los síntomas, los déficits neurocognitivos, la cognición social, los sesgos cognitivos y las experiencias de apego se influyen mútuamente. Sin embargo, poco se sabe si alguna de estas construcciones juega un papel más central que otras a medida que interactúan. Con el objetivo de clarificar esta cuestión, realizamos un análisis de red para explorar la interacción entre un conjunto de variables relacionadas con el apego, los dominios de la cognición y los síntomas psicóticos en una pequeña muestra de persona con estables trastornos del espectro de la esquizofrenia $(n=30)$. Los resultados muestran evidencia preliminar sobre una estructura de red en la que el apego seguro y la psicopatología general son los nódulos más centrales de la red de interacción. Más allá de la importancia de los síntomas, estos resultados preliminares, sugieren la necesidad de evaluar las dimensiones del apego y los dominios cognitivos para diseñar intervenciones específicas para promover la recuperación en personas con trastornos del espectro de la esquizofrenia.

${ }^{7}$ El estudio que se incluye en el presente capítulo ha sido aceptado, con modificaciones, para su publicación en la revista Journal of Psychiatric Research.

Pena-Garijo, J., \& Monfort-Escrig, C. (2021). The centrality of secure attachment within an interacting network of symptoms, cognition, and attachment dimensions in persons with schizophrenia-spectrum disorders: A preliminary study. Journal of Psychiatric Research. In press. https://doi.org/10.1016/ j.jpsychires.2021.01.002 


\title{
The centrality of secure attachment within an interacting network of symptoms, cognition, and attachment dimensions in persons with schizophrenia-spectrum disorders: A preliminary study.
}

\author{
Josep Pena-Garijo ${ }^{a} \mathrm{~b}^{*}$ and Cristina Monfort-Escriga \\ a Jaume I University. Castellon de la Plana (Spain); ${ }^{b}$ Mental Health Service. University Hospital \\ Doctor Peset. Valencia (Spain).
}

\begin{abstract}
Background: Research in the field of psychosis broadly suggests that symptoms, neurocognitive deficits, social cognition, cognitive biases, and attachment experiences influence each other. However, little is known if any of these constructions play a more central role than others as they interact.

Method: To clarify this issue, we conducted a "network" analysis to explore the interplay among a set of variables related to attachment, cognition domains, and psychotic symptoms in a small sample of persons with stabilised schizophreniaspectrum disorders $(n=23)$.

Results: The study provides preliminary evidence about a network structure in which secure attachment and general psychopathology are the most central nodes within the interacting network.

Conclusions: Beyond the importance of symptoms, our results, although preliminary, suggest the need to assess attachment dimensions, and cognition domains to design specific interventions to promote recovery in persons with schizophrenia-spectrum disorders.
\end{abstract}

Keywords: schizophrenia; neurocognition; social cognition; attachment dimensions; network analysis. 


\section{Introduction}

Correlational and theoretical studies in the field of psychosis broadly suggest that symptoms, neurocognitive deficits, social cognition, and cognitive biases influence each other (Pena-Garijo \& Monfort-Escrig, 2020a; 2020b). However, little is known about the paths through which these constructs play a relatively more or less central role than others as they interact (Hasson-Ohayon et al., 2018).

On the other hand, attachment patterns and early-life experiences are relevant to understand the role of environmental factors in the vulnerability and development of psychosis (Bailey et al., 2018; Sheinbaum et al., 2013). Attachment measures correlate with social functioning and interpersonal problems (Berry et al., 2008); are related with social cognition as a mediator between insecure attachment and clinical and functioning problems in psychosis (Korver-Nieberg et al., 2014), and in individuals with psychotic-like experiences (Gawęda et al., 2018). Recent studies about attachment highlight the risk to develop psychotic experiences (Bailey et al., 2018; Rössler et al., 2016) and deficits in social cognitive skills (Rokita et al., 2018).

Despite the large body of research exploring the associations between cognition, psychotic symptoms and attachment styles, more work is required to clarify how they relate to one another (Brown et al., 2014; Korver-Nieberg et al., 2014).

In this sense, "network" approaches improve the limitations of traditional research strategies, while offering promising methodological alternatives (Borsboom \& Cramer, 2013). To date, the "network approach" has been used to explore different psychiatric disorders, including depression, social anxiety, personality disorders and more recently, psychosis (van Rooijen et al., 2017). A recent review of the literature revealed seven studies focusing on psychosisrelated symptoms (Contreras et al., 2019). We found two more studies not included in the review: one exploring the centrality of symptoms, neurocognition, social cognition and metacognition (Hasson-Ohayon et al., 2018), and another 
one assessing the interplay among psychopathology, cognition, functional capacity, and real-life functioning (Galderisi et al., 2018). However, none of these studies included attachment dimensions in the network analysis.

Our study aimed to explore the relative associations among three constructs that have demonstrated to be influent on psychosis: attachment dimensions, cognition domains and psychotic symptoms. For this purpose, we performed a network analysis in a small sample of persons with stabilised schizophrenia-spectrum disorders. Network analysis is a relatively novel approach and has been suggested as an adequate methodology to study mental health problems (Fried et al., 2017), even in small sample sizes (Jones et al., 2018). The inclusion of attachment dimensions in a network analysis is, from our knowledge, a novelty in the field of psychosis.

\section{Methods}

\section{Participants and procedure}

Twenty-three adults diagnosed with a psychotic disorder according to the DSM-5 (APA, 2013) participated voluntarily in the study. Participants were recruited via public mental health centres from Castellon and Valencia (Spain). They were clinically stabilised, with a medication-stabilised condition, and no history of drug abuse in the last four weeks. Fourteen (61\%) had a diagnosis of schizophrenia; 5 (21.7\%), schizoaffective disorder and 4 (17.4\%) were nonspecified psychosis. Nine (39\%) were attended in a rehabilitation centre, and 14 (61\%) were outpatients. The current study is part of a doctorate thesis project approved by Jaume I University (Castellon, Spain) and complied with the Declaration of Helsinki ethical standards. The aim and conditions of the study were verbal and written described to participants, and all of them provided written informed consent. 


\section{Measures}

\section{Attachment dimensions}

We used the reduced Spanish version of CAMIR (from French; Cartes, Models Individueles de Rèlation; Pierrehumbert et al., 1996). Balluerka et al. (2011) developed the reduced version in the Spanish population (CAMIR-R). It is a self-report questionnaire aimed at measuring attachment cognitions. It is based on subjects' evaluations of past and present attachment experiences and family functioning. It evaluates seven factors: Security (availability of the attachment figures), Family concern, Parental interference, Value of parental authority, Parental permissiveness, Self-sufficiency and resentment against parents, and Child traumatism. These factors are related to three attachment styles: secure, preoccupied, and avoidant. In a previous study (unpublished work), Cronbach's alpha coefficients were calculated to test the adequacy of the instrument in a psychotic sample. The results showed that CAMIR-R is reliable for use in persons with psychosis with alpha' coefficients between 0.63 and 0.90 , except for the value of parental authority $(a=0.45)$. Thus, we did not use it in the analyses. Coefficients between 0.63 and 0.90 were considered appropriate for scales of less than eight items and were like those reported by Balluerka et al. (2011) with the Spanish population.

\section{Symptoms}

We used the Positive and Negative Syndrome Scale (PANSS) (Kay et al., 1987; Peralta \& Cuesta, 1994). The measure showed to be reliable with Cronbach's alpha coefficients of 0.73 for the Positive Syndrome Scale, 0.83 for the Negative Syndrome, and 0.79 for the General Psychopathology.

\section{Neurocognition}

The Screen for Cognition Impairment Psychiatric Scale (SCIP-S; Pino et al., 2008) is a neuropsychological test designed for screening of cognitive 
impairments in psychiatric patients. It provides five scores: immediate and delayed verbal learning, working memory, verbal fluency, and processing speed, and an overall score. To avoid an excess of variables, we used the overall score (percentile) to summarise neurocognitive performance.

\section{Theory of Mind}

The Hinting Task (Corcoran et al., 1995) was designed to assess the theory of mind (ToM) in people diagnosed with schizophrenia and provides an overall score for ten or five stories. The Spanish version achieved good internal consistency with alpha coefficients of 0.64 for controls, and 0.69 for participants with psychosis (Gil et al., 2012).

\section{Emotion recognition}

The PERE (from Spanish; Prueba de Evaluación de Reconocimiento de Emociones) includes 56 pictures to evaluate the perception of six basic emotions: joy, sadness, anger, surprise, fear, disgust, and a neutral expression (Gil-Sanz et al., 2017). All photos had an accuracy higher than $89 \%$ and test-retest reliability between 0.80 and 1.32 for controls, and between 0.61 and 1.92 for outpatients. We used the total number of adequately recognised emotions expressions.

\section{Jumping to conclusions bias (JTC)}

The Beads Task is a probabilistic reasoning task in which the subject sees an image of two jars filled with balls of two different colours in different proportions (Dudley \& Over, 2003). A computerised version with a ball's ratio of 60:40 was used. Participants are informed that one of the jars has been randomly selected and the balls will be extracted and displayed one by one on the screen. The task consists of deciding which jar the balls come from, being able to see as many balls as necessary until they are sure of their decision. We used the total number of draws to decide. Less than two draws to decide was used as a threshold to consider a JTC bias (Garety et al., 2011). 


\section{Attributional biases}

The Spanish version of the Attributional Style Questionnaire (ASQ; Peterson et al., 1982; Sanjuán et al., 2009) obtained Cronbach' alpha between 0.74 and 0.80 (Sanjuán at al., 2013). For the current study, we used the selfserving bias, a score that would reflect the tendency to over-attribute positive events to internal causes and adverse events to external causes. An increased selfserving bias is related to delusional experiences (Monfort-Escrig \& Pena-Garijo, 2020).

\section{Data analysis}

Statistical analyses were conducted using the 23.0 version of SPSS, and the 0.13.1 version of JASP (JASP Team, 2020). JASP software included the R Package for network analyses (Rosseel, 2012).

\section{Network analysis}

The partial correlation network shows the remaining association between two nodes after controlling for all other associations (Hasson-Ohayon et al., 2018). The net associations between every two nodes are like regression coefficients (Epskamp et al., 2018). Partial correlations threshold was turned into " 0 " to allow more edges to be estimated. Such estimations tend to include spurious correlations. To minimise it, we used a regularisation technique called LASSO: "least absolute shrinkage and selection operator" (Friedman et al., 2010). LASSO type of network involves the estimation of the model with an extra penalty for complex models and has been used to meet the challenges of small samples in psychology research. The LASSO tuning parameter is typically set between 0 and 0.5. The tuning hyperparameter $\gamma$ of Extended Bayesian Information Criteria was set manually to 0 , to increase the sensitivity of the procedure. It controls the sparsity of the estimated network. Setting it to 0 will cause the regular Bayesian Information Criteria to be used. 
To examine the relative centrality of each construct within the network, we calculated standardised centrality measures. "Betweenness" is measured by calculating how often a variable lies on the shortest path between any combination of two nodes. "Closeness" is the average distance from the node of interest to all other nodes. "Strength" (degree) is the sum of the weighted number and strength of all connections of a specific node to all other nodes (Epskamp et al., 2018).

Finally, to test the reliability of our model, we used some new methods recommended by Epskamp et al. (2018) for estimating psychological networks and their accuracy.

\section{Network construction}

We included three clusters of variables:

Symptoms: positive, negative, and general psychopathology.

Attachment dimensions: security, family concern, parental interference, selfsufficiency, and child traumatism.

Cognition domains: neurocognition, ToM, emotion recognition, and draws to decide (JTC).

We removed permissiveness and self-serving bias from the analysis because they did not show any correlation with the other variables after controlling for all other associations.

The layout of the network was generated via the Fruchterman-Reingold algorithm (Fruchterman \& Reingold, 1991).

\section{Results}

Table 1 shows the characteristics of the participants. All variables included in the analysis were normally distributed, except for the PANSS negative score. The sample was mostly made up of men that were prone to have more symptoms $(r=-0.48 ; p<0.05)$. 
Table 1. Demographic and clinical characteristics of the sample $(N=23)$

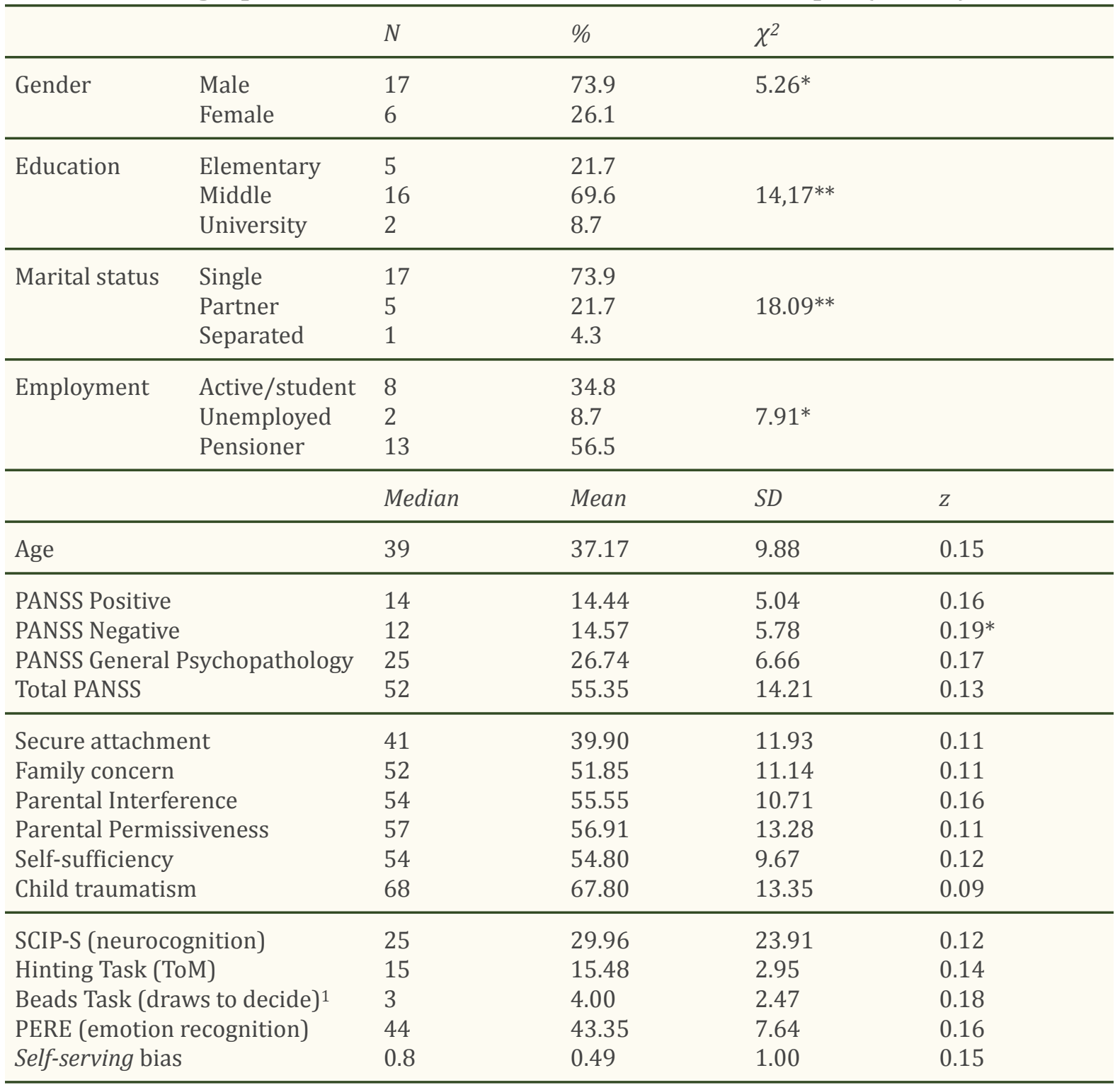

PANSS: Positive and Negative Syndrome Scale. SCIP-S: Screen Cognitive Impairments in Psychiatry-Scale (overall percentile score). $S D$ : Standard Deviation. $\chi^{2}$ : chi-squared test. $Z$ : Kolmogorov-Smirnov test (Lilliefors significance correction).

$* p<0.05$;* $p<0.01$.

${ }^{1}$ A JTC bias (draws to decision $\leq 2$ ) was observed in 7 (30.4\%) participants.

Figure 1 represents the estimated network model. Secure attachment (SA) resulted in the most central node, followed by general psychopathology.

Table 2 shows the weights matrix among the variables included in the network. 


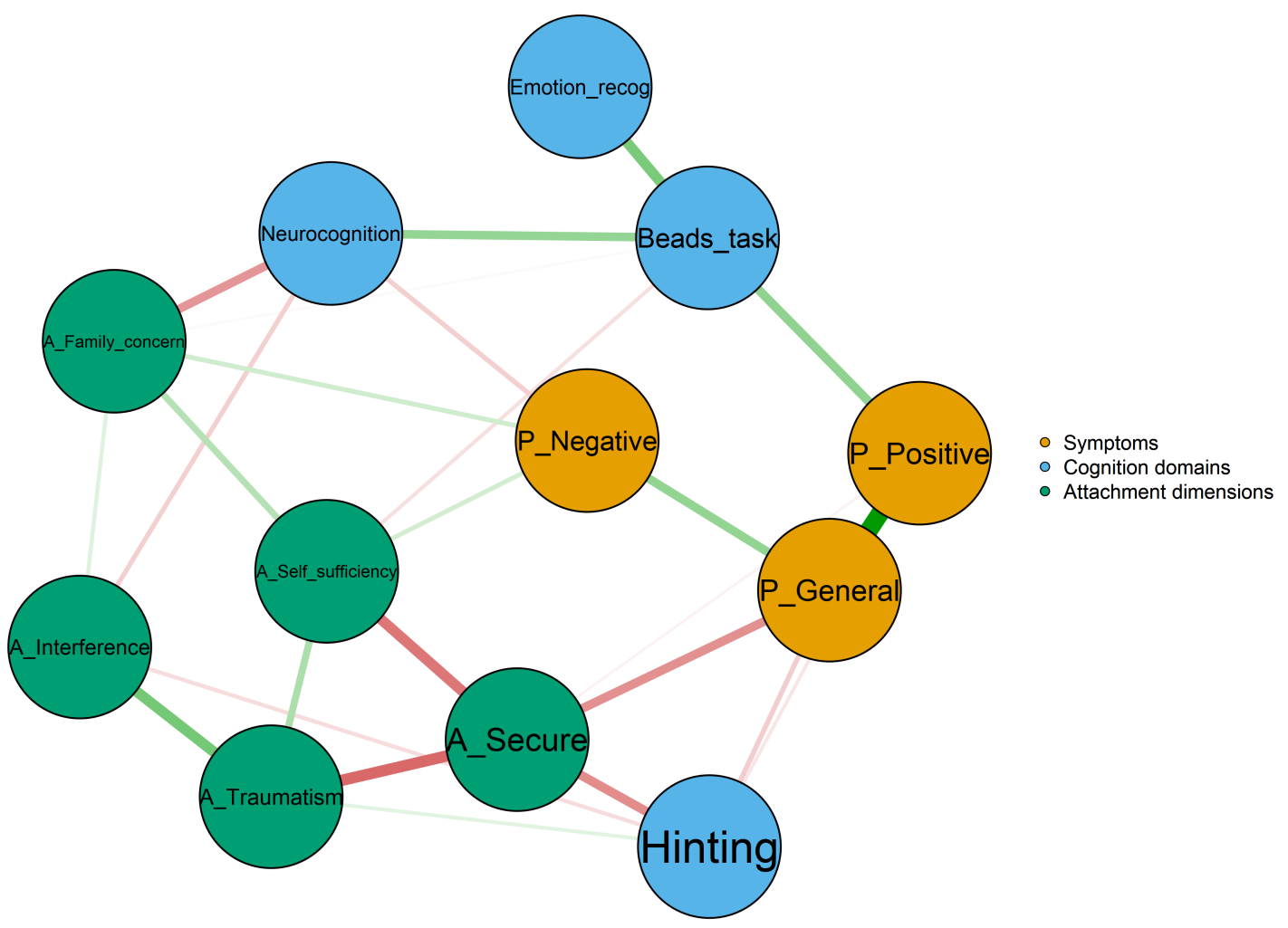

Figure 1. The network model of symptoms, cognition, and attachment dimensions. Each edge within the network corresponds to a partial correlation between two individual items. The thickness of an edge represents the absolute magnitude of the correlation (the thicker the edge, the stronger the connection). Green lines represent positive relations, whereas red ones represent a negative relation. Weights values are shown in Table 2.

Table 2. Weights matrix among the variables included in the network model.

\begin{tabular}{|c|c|c|c|c|c|c|c|c|c|c|c|c|}
\hline & Fam & Inter & Sec & Self & Trau & Neur & Gen & Neg & Pos & Bead & Emo & ToM \\
\hline \multicolumn{13}{|l|}{ Family concern } \\
\hline Interference & 0.069 & & & & & & & & & & & \\
\hline Secure attachment & 0.000 & 0.000 & & & & & & & & & & \\
\hline Self-sufficiency & 0.156 & 0.000 & -0.288 & & & & & & & & & \\
\hline Child Traumatism & 0.000 & 0.291 & -0.317 & 0.175 & & & & & & & & \\
\hline Neurocognition & -0.223 & -0.098 & 0.000 & 0.000 & 0.000 & & & & & & & \\
\hline PANSS General & 0.000 & 0.000 & -0.234 & 0.000 & 0.000 & 0.000 & & & & & & \\
\hline PANSS Negative & 0.100 & 0.000 & 0.000 & 0.092 & 0.000 & -0.102 & 0.228 & & & & & \\
\hline PANSS Positive & 0.000 & 0.000 & -0.027 & 0.000 & 0.000 & 0.000 & 0.536 & 0.000 & & & & \\
\hline Beads Task & -0.012 & 0.000 & 0.000 & -0.006 & 0.000 & 0.224 & 0.000 & 0.000 & 0.234 & & & \\
\hline Emotion recognition & 0.000 & 0.000 & 0.000 & 0.000 & 0.000 & 0.000 & 0.000 & 0.000 & 0.008 & 0.280 & & \\
\hline Theory of Mind & 0.000 & -0.073 & -0.242 & 0.000 & 0.063 & 0.000 & -0.106 & 0.000 & -0.005 & 0.000 & 0.000 & \\
\hline
\end{tabular}

NOTE: Values are standardised coefficients. The partial correlation network shows the remaining association between two nodes after controlling for all other associations. 
Figure 2 shows the centrality measures. These measures are based on the pattern of the connections in which the node of interest plays a role. SA had the best measures, followed by general psychopathology.

Figure 3 represents the edge stability of the network using the Confidence Intervals (95\%) after bootstrapping method (1000 replications). It compares the bootstrap mean with the original sample.

Figure 4 represents the centrality stability of the network. It shows the correlations between the original centrality indices in the whole sample and those estimated in subgroups obtained by dropping increasing percentages of subjects. Centrality indices have an average correlation with the original indices of at least 0.70 with the randomly defined subgroups up to $75 \%$ of case-dropping (i.e., sampled people $=25 \%$ ).
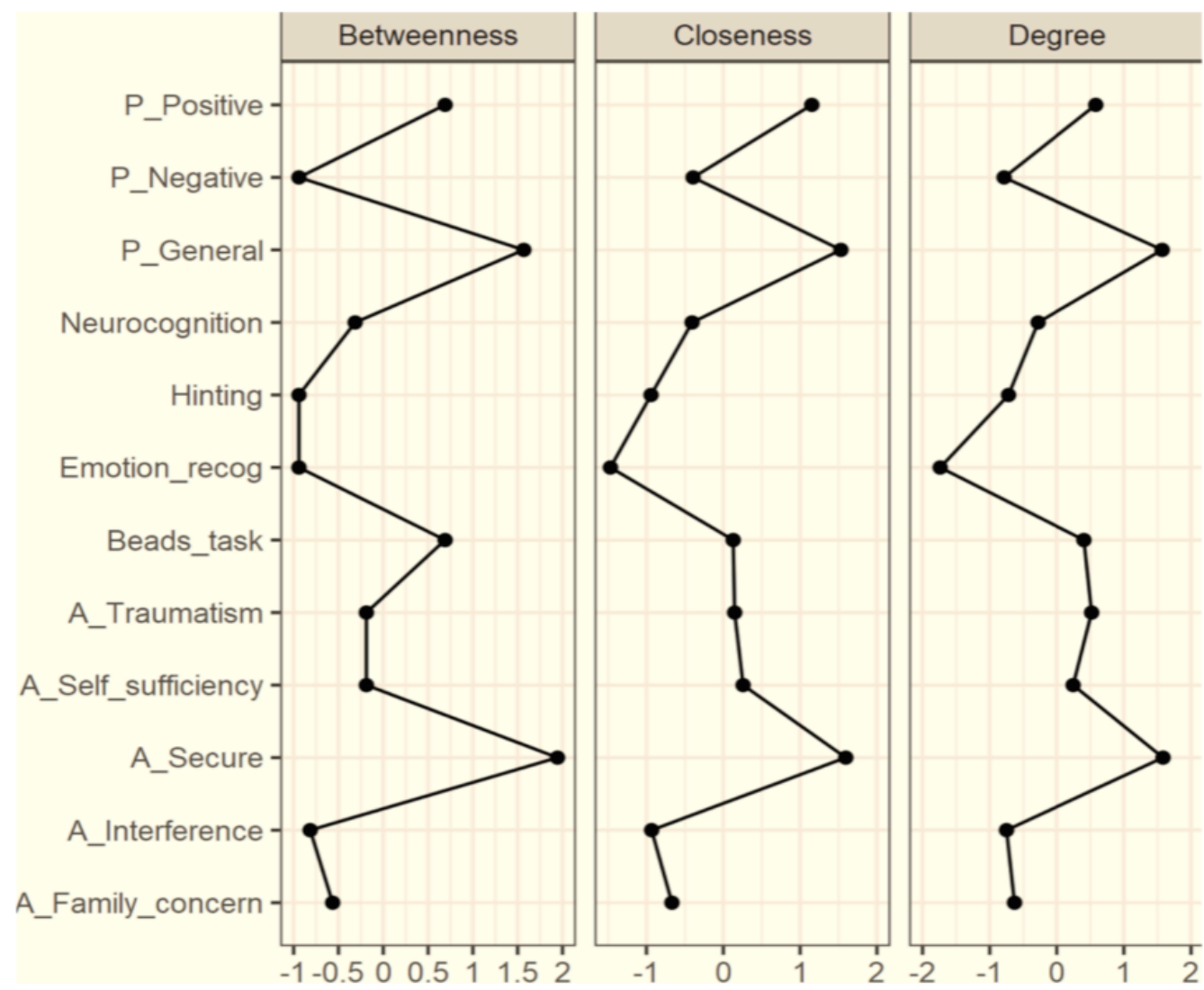

Figure 2. Centrality Plot. Secure attachment is the most influential node, following by PANSS general psychopathology. 


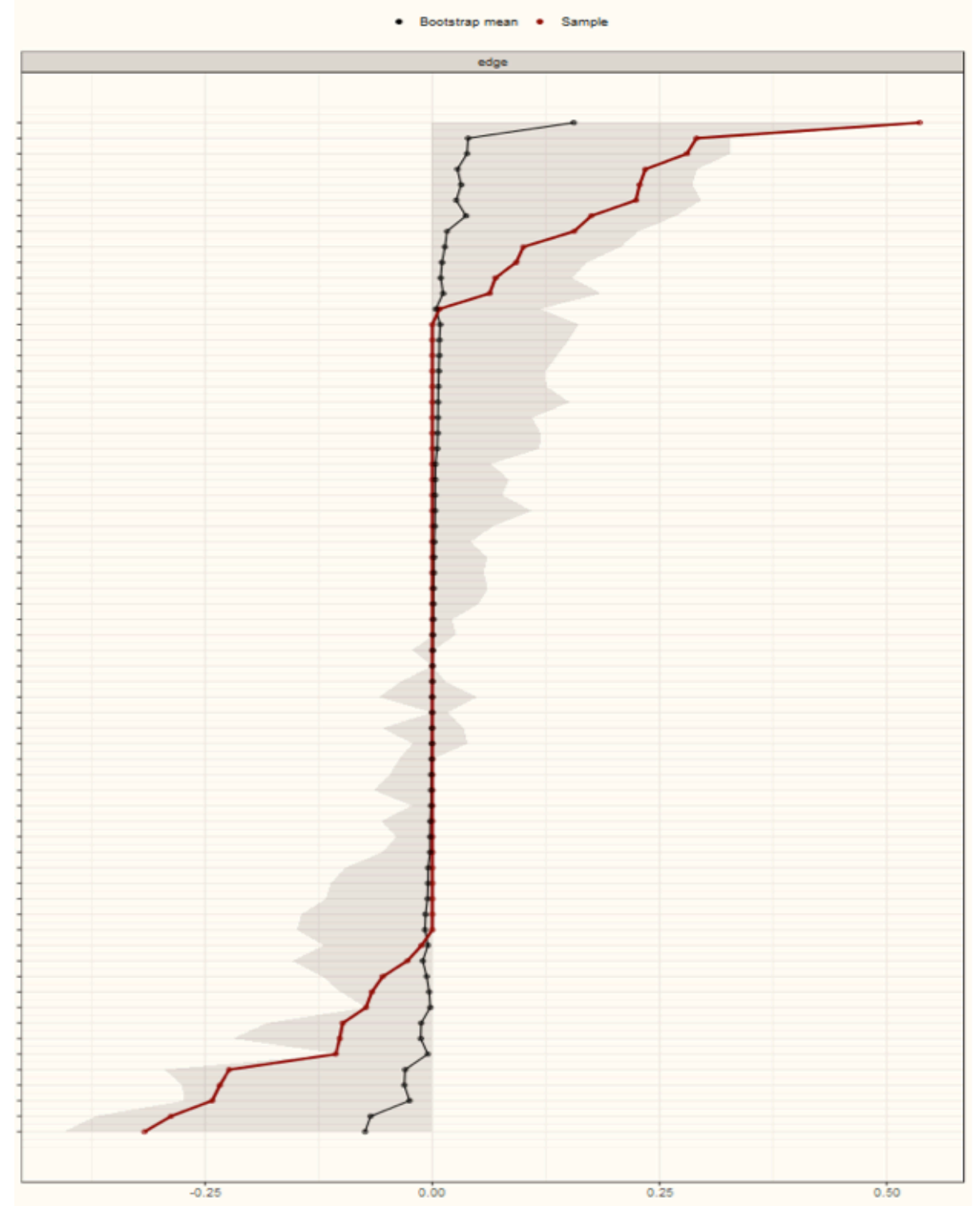

Figure 3. Edge stability. It represents the edge stability of the network using the CIs after bootstrapping method. It compares the bootstrap mean with the original sample. The bootstrapped $\mathrm{CI}$ is the variation that covers $95 \%$ of the cases (1000 replications). Redline represents the original sample. Blackline is the bootstrap mean. The grey area shows the CIs. 


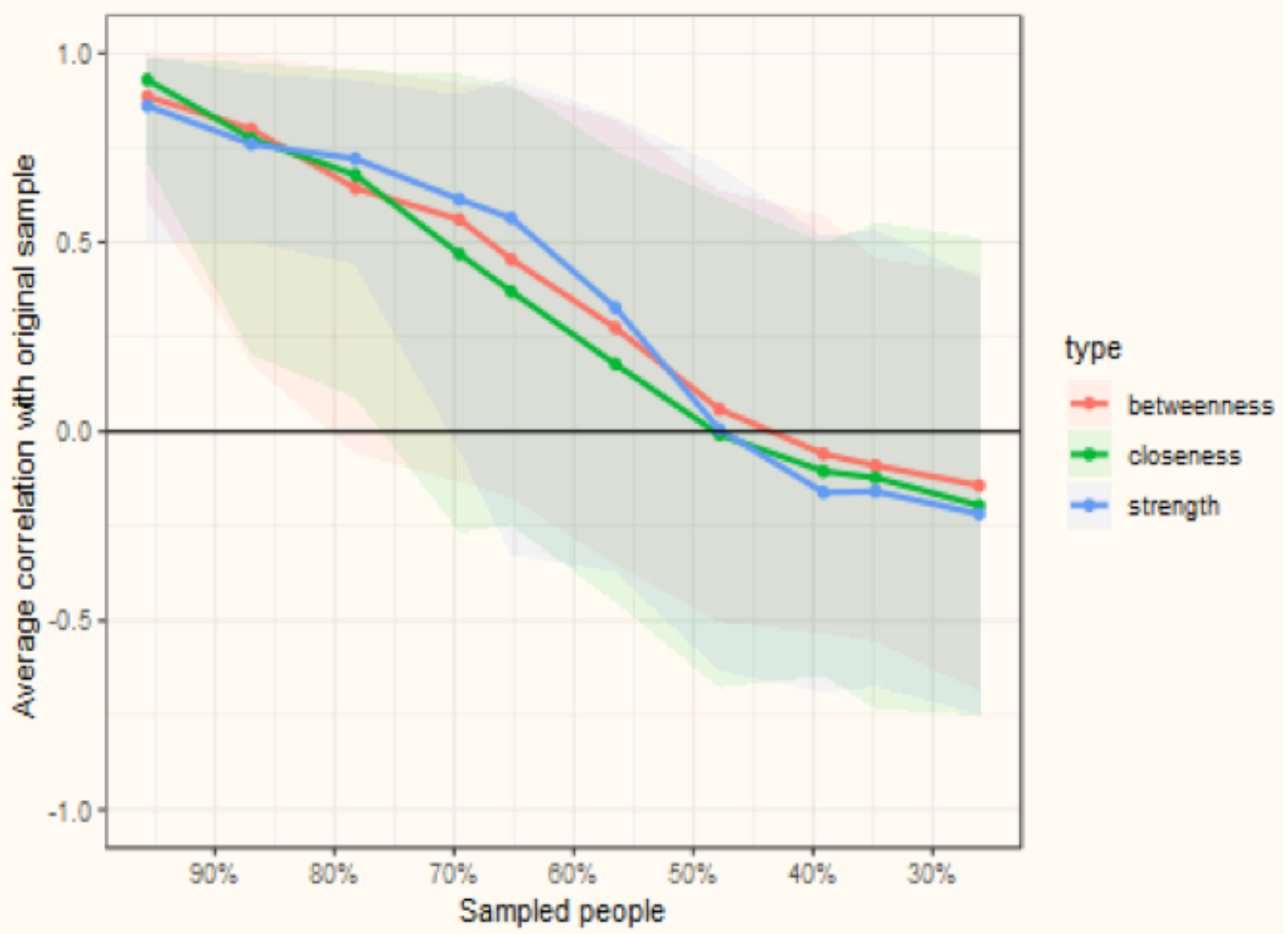

Figure 4. Centrality Stability. Correlations between the original network centrality indices in the whole sample and those estimated in subgroups obtained by dropping increasing percentages of subjects. Average correlations are reported in the $y$-axis and percentages of sampled people in the $x$-axis.

The results should not be interpreted as significance tests, but only as a method to estimate the accuracy of the network generated from the current sample (Epskamp et al., 2018).

\section{Discussion}

Network approaches offer promising methodological alternatives to traditional research strategies and possibilities to guide and evaluate therapeutic interventions (Borsboom \& Cramer, 2013).

The SA appears as the most central node within the interacting network, followed by general psychopathology. SA could be defined as how persons experience availability and support from their attachment figures. Persons with schizophrenia tend to have an insecure attachment style (Bailey et al., 2018), but the associations with symptom dimensions are not clear (Chatziioannidis et al., 
2019). Some works confirm that environmental factors, such as adverse childhood experiences, and daily-life skills are associated with network symptoms in schizophrenia (Galderisi et al., 2018; Isvoranu et al., 2017). In the current report, neurocognition is related to cognitive biases, negative symptoms, and preoccupied attachment (Hasson-Ohayon et al., 2018).

Importantly, SA is negatively correlated to general psychopathology and partially mediates the association between ToM, self-sufficiency (avoidant attachment), and childhood trauma with general psychopathology. As well, family concern (preoccupied) is positively linked to negative symptoms and inversely to neurocognition.

Surprisingly, SA and ToM are inversely associated, contrary to previous literature. We think that this relationship is partially explained because of the characteristics of the sample (from early-psychosis to old patients). Although most of the research shows that the higher SA, the better ToM (Szpak \& Białecka-Pikul, 2020), the relationship between attachment and ToM is likely to change throughout the life-span, particularly between adolescence and adulthood (Hünefeldt et al., 2013).

Lastly, the observed association between beads task and positive symptoms could seem unusual. Beads task is often used to assess the JTC bias regarding its predictive role in the formation of delusional believes, but not necessarily related to "positive syndrome" (e.g. Moritz et al., 2018). Nevertheless, when we analyzed the correlation between beads task and delusions, separated from other positive symptoms, the results showed the association in the expected direction (results not shown). We also find strong associations between beads task and neurocognition and emotion recognition (Garety et al., 2013). Furthermore, over $30 \%$ of participants exhibited a JTC bias, according to previous literature (Dudley et al., 2016). 


\section{Limitations and future research}

Although this is a preliminary report, the small sample size was the main limitation of our study and restricted the number of variables to include in the analysis. Nevertheless, network analysis has been suggested as a good approach to study mental health problems, even using small sample sizes (Jones et al., 2018). Second, separate analyses by gender were not feasible in the current dataset. Lastly, given the novelty of network models in psychopathology, rigorous methods for assessing the reliability of the estimated networks are required. We used some procedures recommended by Epskamp et al. (2018). Results should be interpreted with caution due to the relatively wide confidence intervals in edge stability, although the results in the stability of centrality measures are acceptable. Nevertheless, there is still no agreement on the best indicators of centrality in psychopathology (Bringmann et al., 2019).

Future research should attempt to include more ecological measures, rigorous methods for assessing the reliability of the models, larger and equal samples, relationships with daily-life skills, and a broader set of clinical variables to improve these limitations.

\section{Clinical implications}

Our findings add evidence to the "attachment-developmental-cognitive" hypothesis of schizophrenia (Rajkumar, 2014), that suggests how the attachment may represent a construct capable of bridging different levels of analysis in psychosis.

As well, if SA and general psychopathology are nodes through which other variables interact, specific interventions on them could influence the entire network as they are expected to affect the related nodes (Hasson-Ohayon et al., 2018).

Furthermore, some investigations have observed that secure adult attachment is related to how persons with psychosis engage with mental health services (McGonagle et al., 2019) which is an important clinical issue. 


\section{Conclusions}

Recently, the conceptualization of the dynamic system of psychopathology has gained ground, leading to the development of "network models" (van Rooijen et al., 2017). This report provides preliminary evidence about a network structure in which secure attachment and general psychopathology are central nodes within an interacting network of symptoms, cognition, and attachment dimensions in psychosis.

Beyond the importance of symptoms, our results, although preliminary, suggest the need to assess attachment experiences and cognition domains to improve specific interventions to promote recovery in persons with schizophrenia-spectrum disorders.

\section{References}

American Psychiatric Association. (2013). Diagnostic and statistical manual of mental disorders (5th ed.). Arlington, VA: Author.

Bailey, T., Alvarez-Jimenez, M., Garcia-Sanchez, A. M., Hulbert, C., Barlow, E., \& Bendall, S. (2018). Childhood Trauma Is Associated With Severity of Hallucinations and Delusions in Psychotic Disorders: A Systematic Review and Meta-Analysis. Schizophrenia Bulletin, 44(5), 1111-1122. https://doi.org/10.1093/schbul/sbx161

Balluerka, N., Lacasa, F., Gorostiaga, A., \& Muela, A. (2011). CAMIR-R: Versión Reducida del cuestionario CAMIR. Psicothema, 23, 486-494. Retrieved from http:// www.psicothema.com/psicothema.asp?id=3913

Berry, K., Barrowclough, C., \& Wearden, A. (2008). Attachment theory: A framework for understanding symptoms and interpersonal relationships in psychosis. Behaviour Research and Therapy, 46(12), 1275-1282. https://doi.org/10.1016/j.brat. 2008.08.009

Borsboom, D., \& Cramer, A. O. J. (2013). Network Analysis: An Integrative Approach to the Structure of Psychopathology. Annual Review of Clinical Psychology, 9(1), 91-121. https://doi.org/10.1146/annurev-clinpsy-050212-185608

Bringmann, L. F., Elmer, T., Epskamp, S., Krause, R. W., Schoch, D., Wichers, M., Wigman, J. T. W., \& Snippe, E. (2019). What do centrality measures measure in psychological 
networks? Journal of Abnormal Psychology, 128(8), 892-903. https://doi.org/ $10.1037 / \mathrm{abn} 0000446$

Brown, E. C., Tas, C., Can, H., Esen-Danaci, A., \& Brüne, M. (2014). A closer look at the relationship between the subdomains of social functioning, social cognition and symptomatology in clinically stable patients with schizophrenia. Comprehensive Psychiatry, 55(1), 25-32. https://doi.org/10.1016/j.comppsych.2013.10.001

Chatziioannidis, S., Andreou, C., Agorastos, A., Kaprinis, S., Malliaris, Y., Garyfallos, G., \& Bozikas, V. P. (2019). The role of attachment anxiety in the relationship between childhood trauma and schizophrenia-spectrum psychosis. Psychiatry Research, 276, 223-231. https://doi.org/10.1016/j.psychres.2019.05.021

Contreras, A., Nieto, I., Valiente, C., Espinosa, R., \& Vazquez, C. (2019). The study of psychopathology from the network analysis perspective: A systematic review. Psychotherapy and Psychosomatics, 88, 71-83. https://doi.org/10.1159/000497425

Corcoran, R., Mercer, G., \& Frith, C. D. (1995). Schizophrenia, symptomatology and social inference: Investigating "theory of mind" in people with schizophrenia. Schizophrenia Research, 17(1), 5-13. https://doi.org/10.1016/0920-9964(95)00024-G

Dudley, R. E. J., \& Over, D. E. (2003). People with delusions jump to conclusions: A theoretical account of research findings on the reasoning of people with delusions. Clinical Psychology and Psychotherapy, 10, 263-274. https://doi.org/10.1002/cpp. 376

Dudley, R., Taylor, P., Wickham, S., \& Hutton, P. (2016). Psychosis, Delusions and the "Jumping to Conclusions" Reasoning Bias: A Systematic Review and Metaanalysis. Schizophrenia Bulletin, 42(3), 652-665. https://doi.org/10.1093/schbul/ sbv150

Epskamp, S., Borsboom, D., \& Fried, E. I. (2018). Estimating psychological networks and their accuracy: A tutorial paper. Behavior Research Methods, 50(1), 195-212. https:// doi.org/10.3758/s13428-017-0862-1

Fried, E. I., van Borkulo, C. D., Cramer, A. O. J., Boschloo, L., Schoevers, R. A., \& Borsboom, D. (2017). Mental disorders as networks of problems: a review of recent insights. Social Psychiatry and Psychiatric Epidemiology, 52, 1-10. https://doi.org/10.1007/ s00127-016-1319-z 
Friedman, J., Hastie, T., \& Tibshirani, R. (2010). Applications of the lasso and grouped lasso to the estimation of sparse graphical models. Technical Report, 1-22.

Fruchterman, T. M. J., \& Reingold, E. M. (1991). Graph drawing by force-directed placement. Software: Practice and Experience, 21(11), 1129-1164. https://doi.org/ $10.1002 /$ spe.4380211102

Galderisi, S., Rucci, P., Kirkpatrick, B., Mucci, A., Gibertoni, D., Rocca, P., ... Psychoses, for the I. N. for R. on. (2018). Interplay Among Psychopathologic Variables, Personal Resources, Context-Related Factors, and Real-life Functioning in Individuals With Schizophrenia: A Network Analysis. JAMA Psychiatry, 75(4), 396-404. https:// doi.org/10.1001/jamapsychiatry.2017.4607

Garety, P., Freeman, D., Jolley, S., Ross, K., Waller, H., \& Dunn, G. (2011). Jumping to conclusions: The psychology of delusional reasoning. Advances in Psychiatric Treatment, 17, 332-339. https://doi.org/10.1192/apt.bp.109.007104

Garety, P., Joyce, E., Jolley, S., Emsley, R., Waller, H., Kuipers, E., ... Freeman, D. (2013). Neuropsychological functioning and jumping to conclusions in delusions. Schizophrenia Research, 150(2-3), 570-574. https://doi.org/10.1016/ j.schres.2013.08.035

Gawęda, Ł., Pionke, R., Krężołek, M., Prochwicz, K., Kłosowska, J., Frydecka, D., ... Nelson, B. (2018). Self-disturbances, cognitive biases and insecure attachment as mechanisms of the relationship between traumatic life events and psychotic-like experiences in non-clinical adults - A path analysis. Psychiatry Research, 259, 571-578. https://doi.org/10.1016/j.psychres.2017.11.009

Gil-Sanz, D., Fernández-Modamio, M., Bengochea-Seco, R., Arrieta-Rodríguez, M., González-Fraile, E., Pérez-Fuentes, G., ... Santos-Zorrozúa, B. (2017). PERE: Una nueva herramienta para valorar el reconocimiento de las emociones básicas y su aplicación en la esquizofrenia. Revista de Psicopatologia y Psicologia Clinica, 22(2), 85-93. https://doi.org/10.5944/rppc.vol.22.num.2.2017.17244

Gil, D., Fernández-Modamio, M., Bengochea, R., \& Arrieta, M. (2012). Adaptación al español de la prueba de teoría de la mente Hinting Task. Revista de Psiquiatría y Salud Mental, 5(2), 79-88. https://doi.org/10.1016/j.rpsm.2011.11.004

Hasson-Ohayon, I., Goldzweig, G., Lavi-Rotenberg, A., Luther, L., \& Lysaker, P. H. (2018). The centrality of cognitive symptoms and metacognition within the interacting 
network of symptoms, neurocognition, social cognition and metacognition in schizophrenia. Schizophrenia Research, 202, 260-266. https://doi.org/10.1016/ j.schres.2018.07.007

Hünefeldt, T., Laghi, F., Ortu, F., \& Belardinelli, M. O. (2013). The relationship between 'theory of mind' and attachment-related anxiety and avoidance in Italian adolescents. Journal of Adolescence, 36(3), 613-621. https://doi.org/https://doi.org/10.1016/ j.adolescence.2013.03.012

Isvoranu, A. M., Van Borkulo, C. D., Boyette, L. Lou, Wigman, J. T. W., Vinkers, C. H., Borsboom, D., ... Myin-Germeys, I. (2017). A network approach to psychosis: Pathways between childhood trauma and psychotic symptoms. Schizophrenia Bulletin, 43, 187-196.. https://doi.org/10.1093/schbul/sbw055

JASP Team (2020). JASP (Version 0.13.1). [Computer Software]. https://jasp-stats.org/

Jones, P. J., Mair, P., Riemann, B. C., Mugno, B. L., \& McNally, R. J. (2018). A network perspective on comorbid depression in adolescents with obsessive-compulsive disorder. Journal of Anxiety Disorders, 53, 1-8. https://doi.org/https://doi.org/ 10.1016/j.janxdis.2017.09.008

Kay, S. R., Fiszbein, A., \& Opler, L. A. (1987). The positive and negative syndrome scale (PANSS) for schizophrenia. Schizophrenia Bulletin, 13(2), 261-276. https://doi.org/ $10.1093 /$ schbul/13.2.261

Korver-Nieberg, N., Berry, K., Meijer, C. J., \& De Haan, L. (2014). Adult attachment and psychotic phenomenology in clinical and non-clinical samples: A systematic review. Psychology and Psychotherapy: Theory, Research and Practice, 87, 127-154. https:// doi.org/10.1111/papt.12010

McGonagle, G., Bucci, S., Varese, F., Raphael, J., \& Berry, K. (2019). Is adult attachment associated with engagement with services? A systematic literature review. Journal of Mental Health, 1-12. https://doi.org/10.1080/09638237.2019.1608922

Monfort-Escrig, C., \& Pena-Garijo, J. (2020). Attributional styles and social functioning in schizophrenia. Is the learned helplessness model suitable? Clinica y Salud. Ahead of print. https://doi.org/10.5093/clysa2020a21

Moritz, S., Bentall, R. P., Kolbeck, K., \& Roesch-Ely, D. (2018). Monocausal attribution and its relationship with reasoning biases in schizophrenia. Schizophrenia Research, 193, 77-82. https://doi.org/10.1016/j.schres.2017.06.057 
Pena-Garijo, J., \& Monfort-Escrig, C. (2020a). Cognition in schizophrenia. State of the art (Part I): assessment methods and neural correlates. Revista Asociación Española de Neuropsiquiatría, 40, 109-130. Retrieved from http://www.revistaaen.es/index.php/ aen/article/view/17121

Pena-Garijo, J., \& Monfort-Escrig, C. (2020b). Cognition in schizophrenia. State of the art (Part II): cognitive biases, explanatory models, and intervention programs. Revista Asociación Española de Neuropsiquiatría, 40, 131-154. Retrieved from http:// www.revistaaen.es/index.php/aen/article/view/17122

Peralta, V., \& Cuesta, M. J. (1994). Psychometric properties of the Positive and Negative Syndrome Scale (PANSS) in schizophrenia. Psychiatry Research, 53(1), 31-40. https:// doi.org/10.1016/0165-1781(94)90093-0

Peterson, C., Semmel, A., von Baeyer, C., Abramson, L. Y., Metalsky, G. I., \& Seligman, M. E. P. (1982). The attributional Style Questionnaire. Cognitive Therapy and Research, 6(3), 287-299. https://doi.org/10.1007/BF01173577

Pierrehumbert, B., Pierrehumbert, B., Karmaniola, A., Sieye, A., Meister, C., Miljkovitch, R., \& Halfon, O. (1996). Les modeles de relations: Developpement d'un autoquestionnaire d'attachement pour adultes. Psychiatrie de l'Enfant, 39(1), 161206.

Pino, O., Guilera, G., Rojo, J. E., Gómez-Benito, J., Bernardo, M., Crespo-Facorro, B., ... Rejas, J. (2008). Spanish version of the Screen for Cognitive Impairment in Psychiatry (SCIP-S): Psychometric properties of a brief scale for cognitive evaluation in schizophrenia. Schizophrenia Research, 99(1-3), 139-148. https://doi.org/10.1016/ j.schres.2007.09.012

Rajkumar, R. P. (2014). Childhood attachment and schizophrenia: The "attachmentdevelopmental-cognitive" (ADC) hypothesis. Medical Hypotheses, 83(3), 276-281. https://doi.org/10.1016/j.mehy.2014.05.017

Rokita, K. I., Dauvermann, M. R., \& Donohoe, G. (2018). Early life experiences and social cognition in major psychiatric disorders: A systematic review. European Psychiatry, 53, 123-133. https://doi.org/10.1016/j.eurpsy.2018.06.006

Rosseel, Y. (2012). lavaan: An R Package for Structural Equation Modeling. Journal of Statistical Software, 48(2), 1-36. Retrieved from https://www.jstatsoft.org/v048/i02 
Rössler, W., Ajdacic-Gross, V., Rodgers, S., Haker, H., \& Müller, M. (2016). Childhood trauma as a risk factor for the onset of subclinical psychotic experiences: Exploring the mediating effect of stress sensitivity in a cross-sectional epidemiological community study. Schizophrenia Research, 172(1-3), 46-53. https://doi.org/ 10.1016/j.schres.2016.02.006

Sanjuán, P., Fraguas, D., Magallares, A., \& Merchán-Naranjo, J. (2009). Depressive symptomatology and attributional style in patients with schizophrenia. Clinical Schizophrenia and Related Psychoses, 3(1), 31-38. https://doi.org/10.3371/CSRP. 3.1 .3

Sanjuán, P., Magallares, A., González, J. L., \& Pérez-García, A. (2013). A study on the validity of the Spanish version of the Attributional Style Questionnaire for negative situations. Revista de Psicopatologia y Psicologia Clinica. https://doi.org/10.5944/ rppc.vol.18.num.1.2013.12763

Sheinbaum, T., Bedoya, E., Ros-Morente, A., Kwapil, T. R., \& Barrantes-Vidal, N. (2013). Association between attachment prototypes and schizotypy dimensions in two independent non-clinical samples of Spanish and American young adults. Psychiatry Research, 210(2), 408-413. https://doi.org/10.1016/j.psychres.2013.07.020

Szpak, M., \& Białecka-Pikul, M. (2020). Links between attachment and theory of mind in childhood: Meta-analytic review. Social Development, 29(3), 653-673. https://doi.org/ $10.1111 /$ sode. 12432

van Rooijen, G., Isvoranu, A. M., Meijer, C. J., van Borkulo, C. D., Ruhé, H. G., \& de Haan, L. (2017). A symptom network structure of the psychosis spectrum. Schizophrenia Research, 189, 75-83. https://doi.org/10.1016/j.schres.2017.02.018 


\section{Capítulo 8 Estudio 4}

\section{Predictores del funcionamiento social en personas con esquizofrenia 8}

\section{Resumen}

Los déficits en funcionamiento social (FS) se observan con frecuencia en la esquizofrenia, y se acepta su relación con la cognición y las experiencias de apego. Para explorar cómo estos constructos se ven afectados a través de diferentes condiciones clínicas, realizamos un análisis de varianza (ANOVA) en 45 individuos divididos en tres subgrupos: personas con psicosis estabilizada ( $n=$ 17), psicosis temprana $(n=11)$ y un grupo control $(n=17)$ en diversas dimensiones de la cognición y el apego. A continuación, realizamos un análisis de mediación para probar si los síntomas psicóticos median su influencia sobre el FS dentro del grupo clínico. Se encontraron diferencias significativas que sugieren un continuum de deterioro a través de las tres condiciones. Entre los pacientes, los síntomas mediaron parcialmente la influencia de "teoría de la mente" y del apego seguro sobre el FS, mientras que la neurocognición se vinculó al FS, independientemente de los síntomas. Los resultados sugieren que la cognición y el apego podrían ser importantes objetivos terapéuticos para mejorar el FS de las personas con trastornos del espectro de la esquizofrenia.

\footnotetext{
8 El estudio incluido en el presente capítulo ha sido enviado para su publicación a la revista Behavioural Neurology y se encuentra en proceso de revisión.
} 


\title{
Neurocognition, "theory of mind", and secure attachment predict social functioning in persons with schizophrenia spectrum disorders: A mediation analysis and a comparative study in a sample of the Spanish population
}

\author{
Cristina Monfort-Escrig and Josep Pena-Garijo \\ Jaume I University, Castellón de la Plana, Spain
}

\begin{abstract}
Impairments in social functioning (SF) are frequently seen in schizophrenia, and their relationships with cognition and attachment experiences are commonly accepted. To explore how these constructs are affected across several clinical conditions, we performed a one-way analysis of variance (ANOVA) in forty-five individuals divided into three subgroups: persons with stabilised psychosis $(n=$ $17)$, another one with early psychosis $(n=11)$, and a healthy group $(n=17)$ in cognition and attachment dimensions. Next, we conducted a mediation analysis to test whether psychotic symptoms mediate their influence on SF, within the clinical group. Significant differences were found, suggesting a continuum of impairment across the three conditions. Within patients, symptoms partially mediated the influence of "theory of mind" and secure attachment on SF, whereas neurocognition was linked to SF, regardless of symptoms. The results suggest that cognition and attachment could be important therapeutic targets to improve SF in persons with schizophrenia spectrum disorders.
\end{abstract}

Keywords: Schizophrenia. Social functioning. Cognition. Attachment. Earlypsychosis. Mediation analysis. 


\section{Introduction}

Relevant studies in the field of psychosis broadly suggest that symptoms, neurocognitive deficits, social cognition, and cognitive biases influence each other (Pena-Garijo \& Monfort-Escrig, 2020a; 2020b). Moreover, "theory of mind" (ToM), emotion recognition, reasoning biases, and attributional styles have demonstrated to be influent in clinical and social outcomes in psychosis-related disorders (Browne et al., 2016; Buck et al., 2016; Mallawaarachchi et al., 2019), as well as neurocognition (Bora et al., 2017).

On the other hand, attachment patterns and early-life experiences are relevant to understand the role of psychosocial and environmental factors in the vulnerability and development of psychosis (Bailey et al., 2018; Rokita et al., 2018; Sheinbaum et al., 2013). Attachment measures are related with social cognition as a mediator between insecure attachment and clinical and functioning problems in psychosis (Korver-Nieberg et al., 2014), and in individuals with psychotic-like experiences (Gawęda et al., 2018).

Despite the vast body of research exploring the associations between cognition, psychotic symptoms, and attachment styles, more work is required to clarify how they are associated with social functioning (SF) (Brown et al., 2014; Korver-Nieberg et al., 2014), as well as how they are affected compared with the healthy population, or across different clinical conditions. For instance, several studies report impairments in social cognition across the psychosis continuum and phases of illness (Browne et al., 2016; Kamel \& AlQahtani, 2019; Ludwig et al., 2017).

To clarify these issues, we conducted two separate studies. To explore how SF, neurocognition, social cognition, and attachment dimensions are affected across several clinical conditions, we performed a one-way analysis of variance (ANOVA) in a sample of forty-five Spanish people distributed into three subgroups: a group of persons with stabilised psychosis, another one with earlypsychosis episodes, and a healthy control one. After, we conducted a mediation analysis within the clinical group to investigate how symptoms, cognition 
domains, and attachment dimensions influence a measure of SF by using structural equation modelling (SEM) (Rosseel, 2012). Mediation and pathway analyses have been used to study possible paths between cognition and symptoms (e.g., Lam et al., 2014), and between attachment and psychotic experiences (Gawęda et al., 2018; Korver-Nieberg et al., 2014). We expected to replicate previous studies in the field of psychosis, demonstrating that persons with psychotic disorders will show significant impairments in SF, neurocognition, social cognition, and attachment dimensions compared with the healthy population. Moreover, we hypothesised a possible "gradation" of deficits across the three studied conditions. Finally, although we considered mediation analysis to be partially exploratory, we hypothesised that the influence of attachment and cognition on SF would be partially mediated by symptom severity.

\section{Methods}

\section{Participants and procedure}

Forty-five adults participated voluntarily in the study. The clinical group was composed of twenty-eight persons diagnosed with a psychotic disorder according to the DSM-5 (American Psychiatric Association, 2014). Participants were recruited via public mental health centres from Castellon and Valencia (Spain). They were clinically stabilised, with a medication-stabilised condition, and with no history of drug abuse in the last four weeks. Nine $(32.1 \%)$ were attended in a day rehabilitation centre, and 19 (67.9\%) were outpatients. The nonclinical group involved 17 individuals with no history of mental health problems, recruited as a convenience sample (age and gender) in the same geographic region. The current study is part of a doctorate thesis approved by the Jaume I University Deontological Committee and complied with the Declaration of Helsinki ethical standards. The aims and conditions of the study were verbal and written described to prospective participants, and all of them provided written informed consent. 


\section{Instruments}

To assess attachment dimensions, we used the reduced Spanish version of CAMIR (from French; Cartes, Models Individueles de Rèlation) (Pierrehumbert et al., 1996). The CAMIR-R (Balluerka et al., 2011) is a self-report questionnaire aimed at measuring attachment cognitions. It is based on subjects' evaluations of past and present attachment experiences and family functioning. It consists of 32 items rated on a five-point Likert-type scale and evaluates seven factors: Security (availability and support of the attachment figures), Family concern, Parental interference, Value of parental authority, Parental permissiveness, Selfsufficiency, and resentment against parents, and Child traumatism. These factors are related to three attachment styles: secure, preoccupied, and avoidant. CAMIR$\mathrm{R}$ dimensions provide a valid and reliable assessment of attachment representations and the family functioning conception (Balluerka et al., 2011). In a previous study, Cronbach's alpha coefficients were calculated to test the adequacy of the instrument in the psychotic population (Monfort-Escrig \& PenaGarijo, unpublished work). The results showed that CAMIR-R is reliable for use in people with psychosis with alphas' coefficients between 0.63 and 0.90 except for the value of parental authority ( $a=0.45$ ). Thus, we did not use it in the analyses. The coefficients between 0.63 and 0.90 were considered appropriate for scales of less than eight items and were like those reported by Balluerka et al. (2011) with the Spanish population.

We used the Positive and Negative Syndrome Scale (PANSS) (Kay et al., 1987; Peralta \& Cuesta, 1994) to assess symptom severity. The measure showed to be reliable with Cronbach's alpha coefficients of 0.73 for the Positive Syndrome Scale, 0.83 for the Negative Syndrome Scale, and 0.79 for the General Psychopathology Scale (Kay et al., 1987).

Neurocognition was evaluated through the Screen for Cognition Impairment Psychiatric Scale (SCIP-S; Pino et al., 2008). It is a short neuropsychological test designed for screening of cognitive impairment in psychiatric patients. The test provides scores for five factors: immediate and 
delayed verbal learning, working memory, verbal fluency, and processing speed, and an overall score. To avoid an excess of variables, we used the total percentile score to summarize neurocognitive performance.

We selected the Spanish version of the Hinting Task to evaluate "the theory of mind" (ToM) (Corcoran et al., 1995; Gil et al., 2012). It was explicitly designed to assess ToM in people diagnosed with schizophrenia and provides an overall score for ten stories, and another one for five stories. The Spanish version achieved good internal consistency with alpha coefficients of 0.64 for controls and 0.69 for participants with psychosis (Gil et al., 2012).

The PERE (from Spanish; Prueba de Evaluación de Reconocimiento de Emociones) includes 56 pictures (28 men and 28 women) to evaluate the perception of six basic emotions: joy, sadness, anger, surprise, fear, disgust, and a neutral expression (Gil-Sanz et al., 2017). All photos had an accuracy higher than $89 \%$ and test-retest reliability between 0.80 and 1.32 for controls, and between 0.61 and 1.92 for outpatients. We used the total number of correctly recognized emotion expressions as an emotional recognition indicator.

The Beads Task is a probabilistic reasoning task in which the subject sees an image of two jars filled with balls of two different colours in different proportions (Dudley \& Over, 2003). A computerized version with a ball's ratio of 60:40 was used. Participants are informed that one of the jars has been randomly selected and the balls will be extracted from it and displayed one by one on the screen. The task consists of deciding which jar the balls come from, being able to see as many balls as necessary until they are entirely sure of their decision. Less than two draws to decide is often used as a threshold to consider a jumping to conclusions (JTC) bias (Garety et al., 2011).

The Spanish version of the Attributional Style Questionnaire (ASQ; Peterson et al., 1982; Sanjuán et al., 2009) obtained Cronbach' alpha between 0.74 and 0.80 (Sanjuán et al., 2013). In the current study, we used the self-serving bias, a score that would reflect the tendency to overattribute positive events to internal causes and adverse events to external causes. An increased self-serving 
bias is related to delusional experiences (Candido \& Romney, 1990; cited by Moritz et al., 2018). Additionally, we used the composite index, a score that reflects an "optimistic-pessimistic" attributional style and has demonstrated to be related to depressive symptoms in schizophrenia (Monfort-Escrig \& PenaGarijo, in press).

To assess social functioning, we used the reduced Spanish version of the Social Functioning Scale (SFS-R; Alonso et al., 2008). SFS (Torres \& Olivares, 2005) was designed to evaluate SF essential to schizophrenia through a clinical interview. The original 78 items were reduced to 15 . The SFS-R assesses five areas (social isolation, interpersonal communication, independence execution, independence-competence, and employment occupation), and allows a total score. The short form had a Cronbach' alpha of 0.76 and proved to be reliable for use in clinical practice. In our study, we obtained a Global SF score by summing the current employment occupation status (scores from 1 to 3 ) to the overall SFS$\mathrm{R}$ score (excluding the original employment occupation items).

\section{Statistical analysis}

The statistical analyses were conducted using the 23.0 version of SPSS and the 0.13.1 version of JASP (JASP Team, 2020). JASP software included the $\mathrm{R}$ Package for mediation analysis and structural equation modelling (Rosseel, 2012).

\section{Comparisons between groups}

We conducted a one-way ANOVA to compare the three groups. Given the small sample size, unequal groups, and considering that several variables were not normally distributed, we decided to use nonparametric tests. The KruskalWallis test was used to check significant differences between groups. As the assumption of homogeneity was not met, Welch correction was used, and partial $\eta 2$ (partial eta-square) was selected as an estimate of the effect size. For post hoc comparisons, Dunn correction was applied. It is a nonparametric test that can be 
used for testing small subsets of pairs. This post hoc test is a follow-up for the Kruskal-Wallis test. It provides its own p-value, and p-values corrected with the Bonferroni and Holm methods.

\section{Mediation analysis}

To test whether symptoms would be a possible mediator between cognition and attachment and SF, we conducted two correlative analyses within the clinical sample $(n=23)$. First, we performed a linear regression analysis to explore the possible predictors of SF, entering all variables as independent variables to be considered in the model (except symptom dimensions). To avoid collinearity problems with the enter method, we used the backward method to remove one by one all variables that did not get enough significance in each step, until we obtained a final model in which all remaining variables were statistically significant $(\mathrm{p}<0.05)$ in predicting the dependent variable $(\mathrm{SF})$. Next, we conducted a mediation analysis to determine the direct and indirect effects of attachment dimensions and cognition domains to predict SF through symptom severity as the hypothesised mediator. Mediation analysis in JASP is described by Rosseel (2012). We introduced all variables suggested by the regression model (neurocognition, ToM, secure attachment, and parental interference) as predictors; symptom severity (PANSS total score) was introduced as the mediator variable and $\mathrm{SF}$ as the predicted variable. Command options to calculate estimates were turned into standardised estimates, and missing values were handled as Full Information of Maximum Likelihood. For testing whether indirect effects exist, we used the bootstrap method (1000 replications). The Confidence Intervals (CI) were then computed using the bias-corrected percentile method, as suggested by Biesanz et al. (2010). The pathway analysis was conducted with SEM by using the lavaan syntax (Rosseel, 2012). We verified the goodness of model fit following the guidelines from literature (Hu \& Bentler, 1999): RMSEA < 0.06 (Root Mean Square Error of Approximation); SRMR $<0.08$ (Standardised 
Root Mean Square Residual); CFI > 0.95 (Confirmatory Fit Index); GFI > 0.95 (Goodness of Fit Index) and TLI > 0.95 (Tucker-Lewis Index).

Table 1. Demographic and clinical characteristics of the sample $(N=45)$

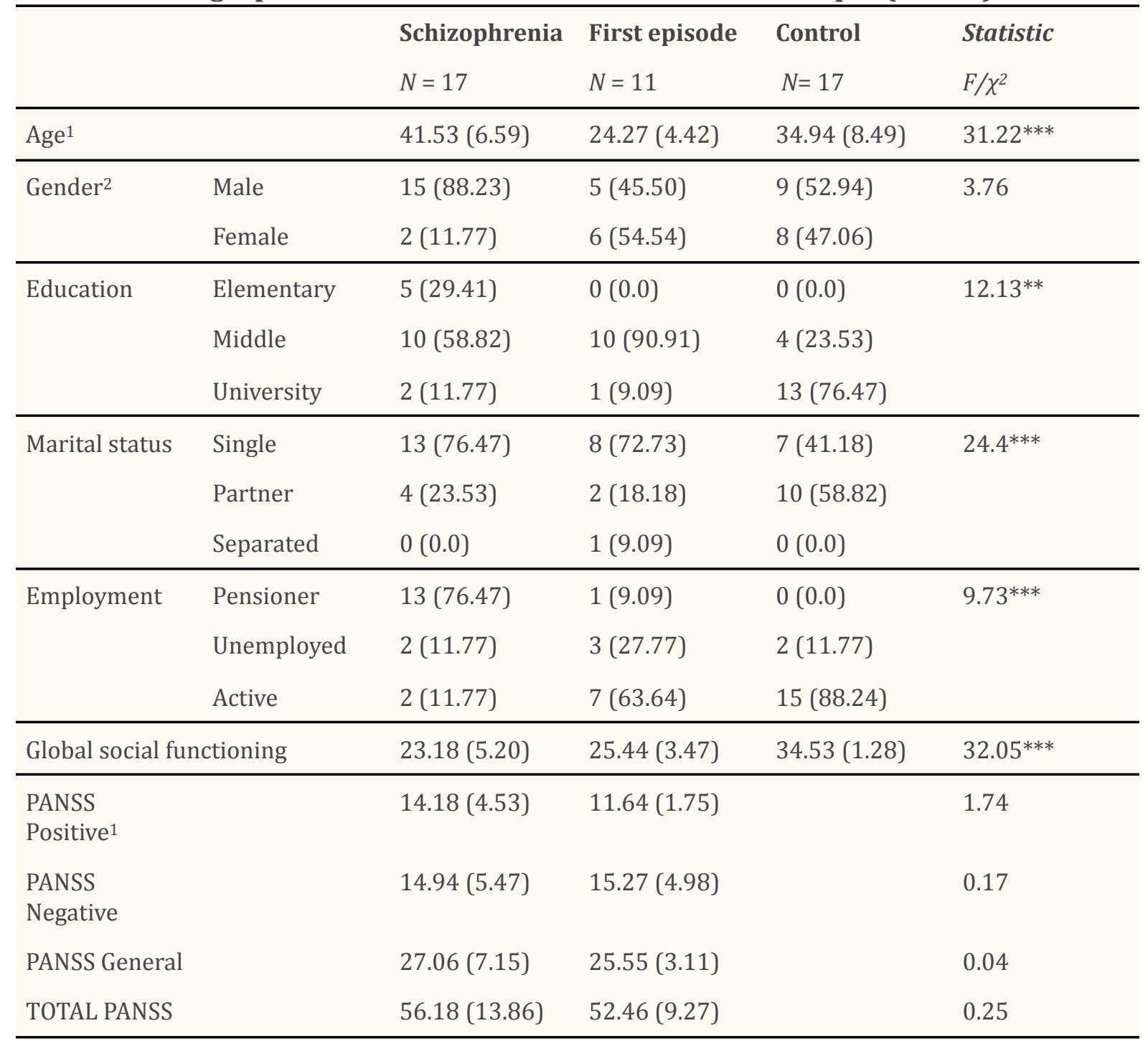

${ }^{1}$ Mean (Standard deviation). ${ }^{2}$ Frequency (\%). PANSS. Positive and Negative Syndrome Scale.

${ }^{*} p<0.01 ; * * p 0.001$

\section{Results}

No differences in gender $(W=194.00 ; p=0.21)$ and age $(W=237.50 ; p=$ $0.99)$ were found between the clinical sample $(n=28)$ and the control one $(n=$ 17). However, when we compared the three separated subgroups, differences in age appeared to be statistically significant. No differences in PANSS dimensions were found between the two clinical conditions (see Table 1). 
Table 2. Comparisons between groups. Summary of ANOVA

\begin{tabular}{|c|c|c|c|c|c|c|c|}
\hline Variable & Group & Mean & SD & Post hoc comparisons & $z$ & $\boldsymbol{F}$ & $p n^{2}$ \\
\hline \multicolumn{8}{|l|}{ Cognition domains } \\
\hline \multirow[t]{3}{*}{ Neurocognition } & Control & 89.47 & 10.24 & Schizophrenia - First episode & $-1.67^{*}$ & & \\
\hline & First episode & 50.09 & 25.76 & Schizophrenia - Control & $-5.49 * * *$ & $30.84 * * *$ & 0.69 \\
\hline & Schizophrenia & 25.59 & 22.19 & First episode - Control & $-3.19^{* * *}$ & & \\
\hline \multirow[t]{3}{*}{ Beads task } & Control & 14.59 & 4.17 & Schizophrenia - First episode & -0.98 & & \\
\hline & First episode & 5.00 & 2.83 & Schizophrenia - Control & $-5.41^{* * *}$ & $31.72^{* * *}$ & 0.74 \\
\hline & Schizophrenia & 3.41 & 1.91 & First episode - Control & $-3.81^{* * *}$ & & \\
\hline \multirow[t]{3}{*}{ Theory of Mind } & Control & 19.94 & 0.24 & Schizophrenia - First episode & 1.02 & & \\
\hline & First episode & 14.55 & 2.42 & Schizophrenia - Control & $-4.61^{* * *}$ & $32.96^{* * *}$ & 0.52 \\
\hline & Schizophrenia & 16.03 & 3.03 & First episode - Control & $-5.11^{* * *}$ & & \\
\hline \multirow{3}{*}{$\begin{array}{l}\text { Emotion } \\
\text { recognition }\end{array}$} & Control & 53.59 & 1.70 & Schizophrenia - First episode & $-2.02^{*}$ & & \\
\hline & First episode & 48.46 & 3.05 & Schizophrenia - Control & $-5.23 * * *$ & $28.22^{* * *}$ & 0.53 \\
\hline & Schizophrenia & 41.47 & 7.82 & First episode - Control & $-2.68^{* *}$ & & \\
\hline \multirow[t]{3}{*}{ Optimistic style } & Control & 5.53 & 1.58 & Schizophrenia - First episode & $-1.91^{*}$ & & \\
\hline & First episode & 3.33 & 1.62 & Schizophrenia - Control & $-5.01^{* * *}$ & $25.19 * * *$ & 0.50 \\
\hline & Schizophrenia & 1.23 & 2.36 & First episode - Control & $-2.39 * *$ & & \\
\hline \multicolumn{8}{|c|}{ Attachment dimensions } \\
\hline \multirow[t]{3}{*}{ Secure attachment } & Control & 53.45 & 6.38 & Schizophrenia - First episode & -1.19 & & \\
\hline & First episode & 44.14 & 15.56 & Schizophrenia - Control & $-3.76^{* * *}$ & $14.15^{* * *}$ & 0.34 \\
\hline & Schizophrenia & 38.65 & 11.30 & First episode - Control & -1.35 & & \\
\hline \multirow[t]{3}{*}{ Family concern } & Control & 39.58 & 11.69 & Schizophrenia - First episode & $2.63^{* *}$ & & \\
\hline & First episode & 39.56 & 11.55 & Schizophrenia - Control & $3.63^{* * *}$ & $15.41^{* * *}$ & 0.38 \\
\hline & Schizophrenia & 55.47 & 8.72 & First episode - Control & -0.19 & & \\
\hline \multicolumn{8}{|c|}{ Social functioning areas } \\
\hline \multirow[t]{3}{*}{ Social isolation } & Control & 5.88 & 0.33 & Schizophrenia - First episode & -0.49 & & \\
\hline & First episode & 3.76 & 0.73 & Schizophrenia - Control & $-5.24 * * *$ & $31.71^{* * *}$ & 0.67 \\
\hline & Schizophrenia & 3.41 & 1.18 & First episode - Control & $-4.16^{* * *}$ & & \\
\hline \multirow{3}{*}{$\begin{array}{l}\text { Interpersonal } \\
\text { comm. }\end{array}$} & Control & 9.18 & 0.73 & Schizophrenia - First episode & 0.09 & & \\
\hline & First episode & 5.59 & 1.61 & Schizophrenia - Control & $-4.85^{* * *}$ & $29.69^{* * *}$ & 0.59 \\
\hline & Schizophrenia & 5.59 & 1.97 & First episode - Control & $-4.38^{* * *}$ & & \\
\hline \multirow{3}{*}{$\begin{array}{l}\text { Independence- } \\
\text { exec. }\end{array}$} & Control & 8.94 & 0.24 & Schizophrenia - First episode & 0.09 & & \\
\hline & First episode & 7.18 & 0.55 & Schizophrenia - Control & $-4.57^{* * *}$ & $25.97^{* * *}$ & 0.44 \\
\hline & Schizophrenia & 6.41 & 2.06 & First episode - Control & $-4.06^{* * *}$ & & \\
\hline \multirow{3}{*}{$\begin{array}{l}\text { Independence- } \\
\text { comp. }\end{array}$} & Control & 7.65 & 0.70 & Schizophrenia - First episode & -0.55 & & \\
\hline & First episode & 6.86 & 1.21 & Schizophrenia - Control & $-2.74^{* *}$ & $8.06^{*}$ & 0.16 \\
\hline & Schizophrenia & 6.41 & 1.73 & First episode - Control & $-1.88^{*}$ & & \\
\hline
\end{tabular}

Only comparisons with significant differences in the Kruskal-Wallis test are shown. Z-scores are values in Dunn post hoc tests. $F$ is the Kruskal-Wallis statistic. Partial $n^{2}$ represents the effect sizes.

${ }^{*} p<0.05 ;{ }^{* *} p<0.01 ;{ }^{* * *} p<0.001$. 

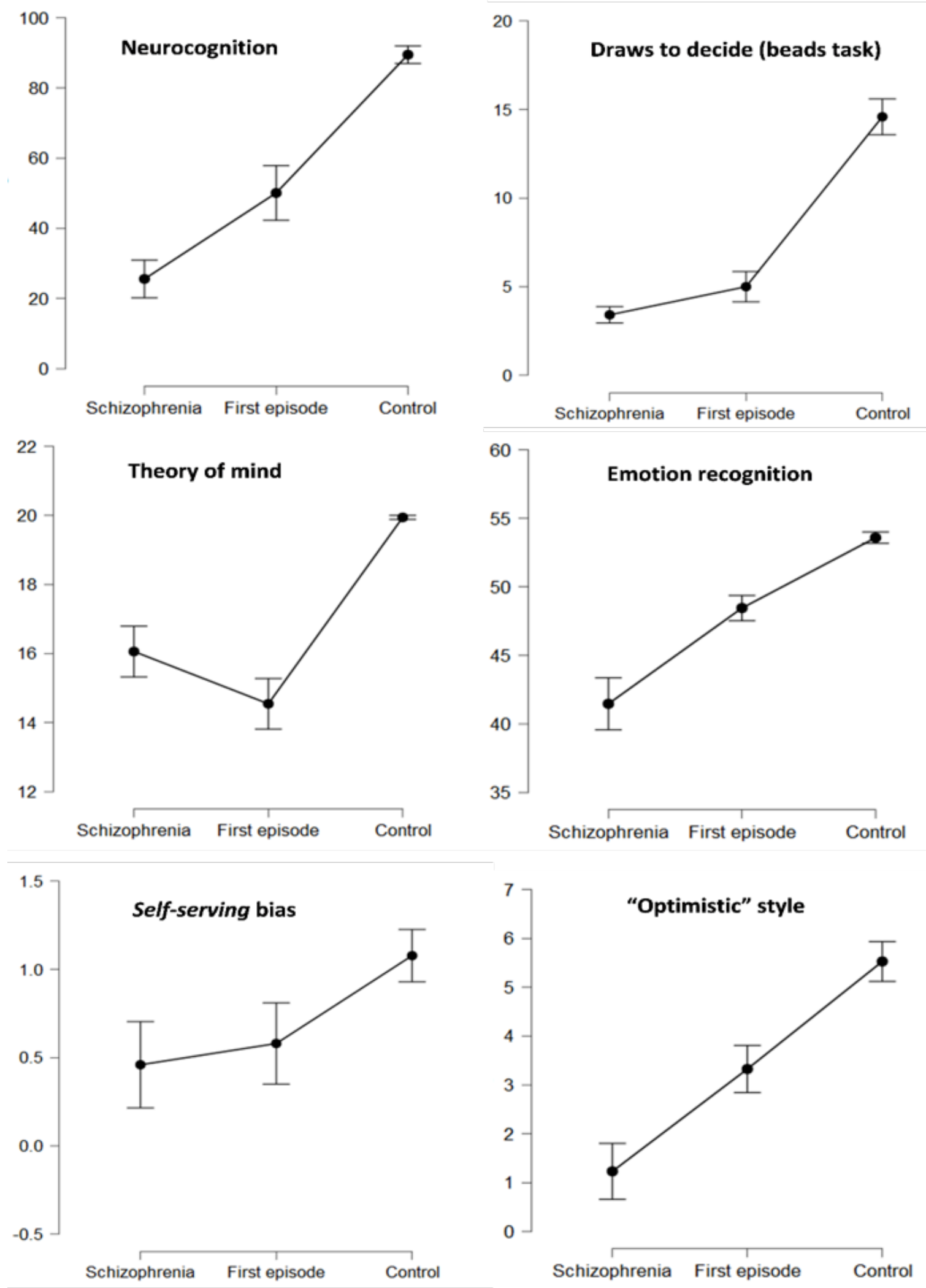

Figure 1. Comparisons between groups in cognition domains. Bars represent standard errors. 

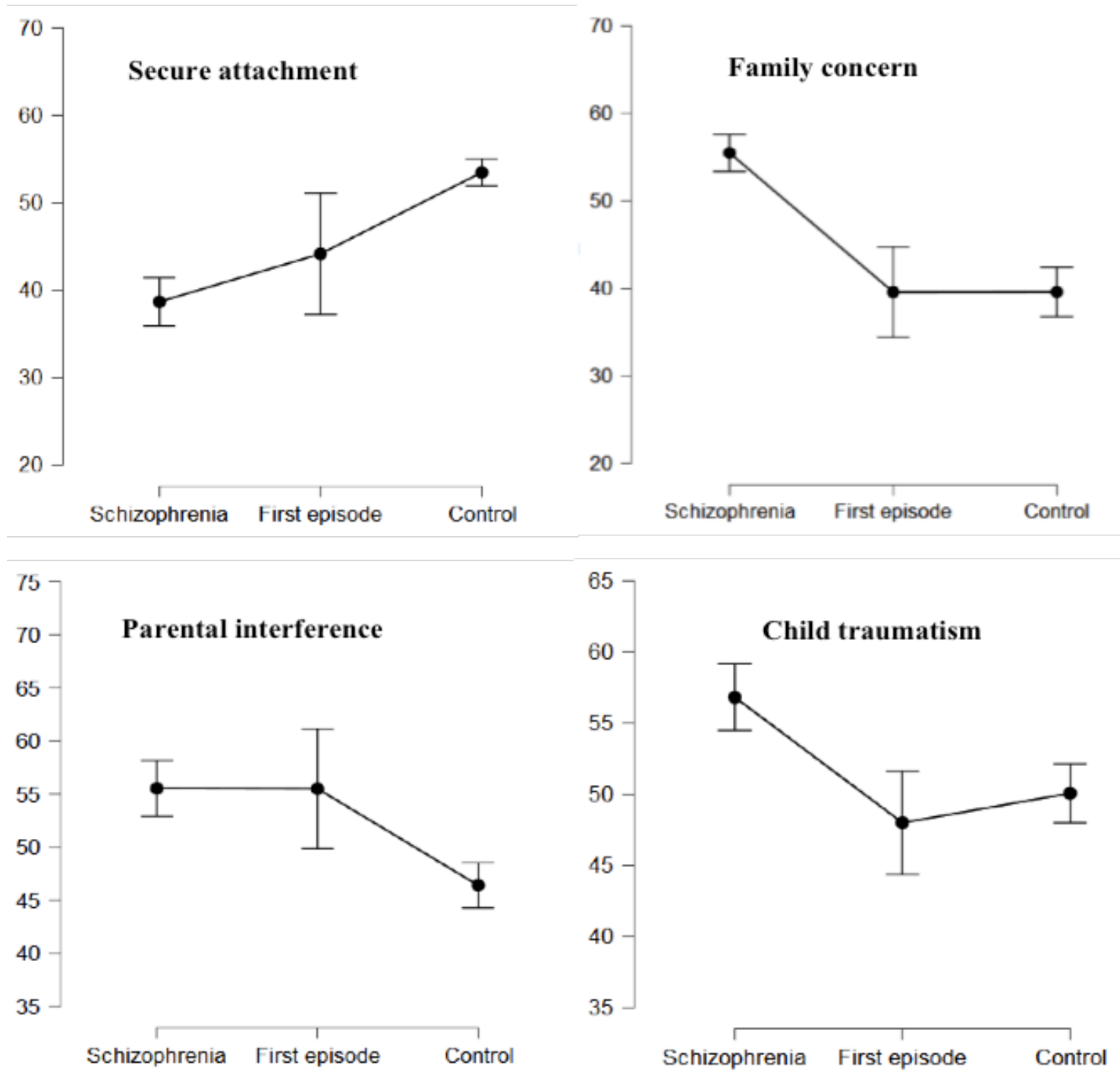

Figure 2. Comparisons between groups in attachment dimensions. Bars represent standard error.

\section{Differences between groups}

The differences between groups were significant for neurocognition, ToM, "draws to decide" (beads task), "optimistic" style (ASQ composite), and emotion recognition with large effect sizes (all partial $\eta^{2}>0.45 ; p<0.001$ ) (see Table 2). Secure attachment and family concern (related to preoccupied attachment) also differentiated between groups (partial $\eta^{2}>0.30 ; p<0.001$ ) (see Figure 1). Social functioning was more impaired in both clinical groups than in the healthy one with a large effect size (partial $\eta^{2}=0.67 ; p<0.001$ ) (see Figure 2). The differences between the stabilised psychosis group and the control one were significant $(z=-5.43 ; p<0.001)$, as well as between the first episode and control 
$(z=-3.83 ; p<0.001)$. No differences were found between both clinical conditions (see Figure 3).
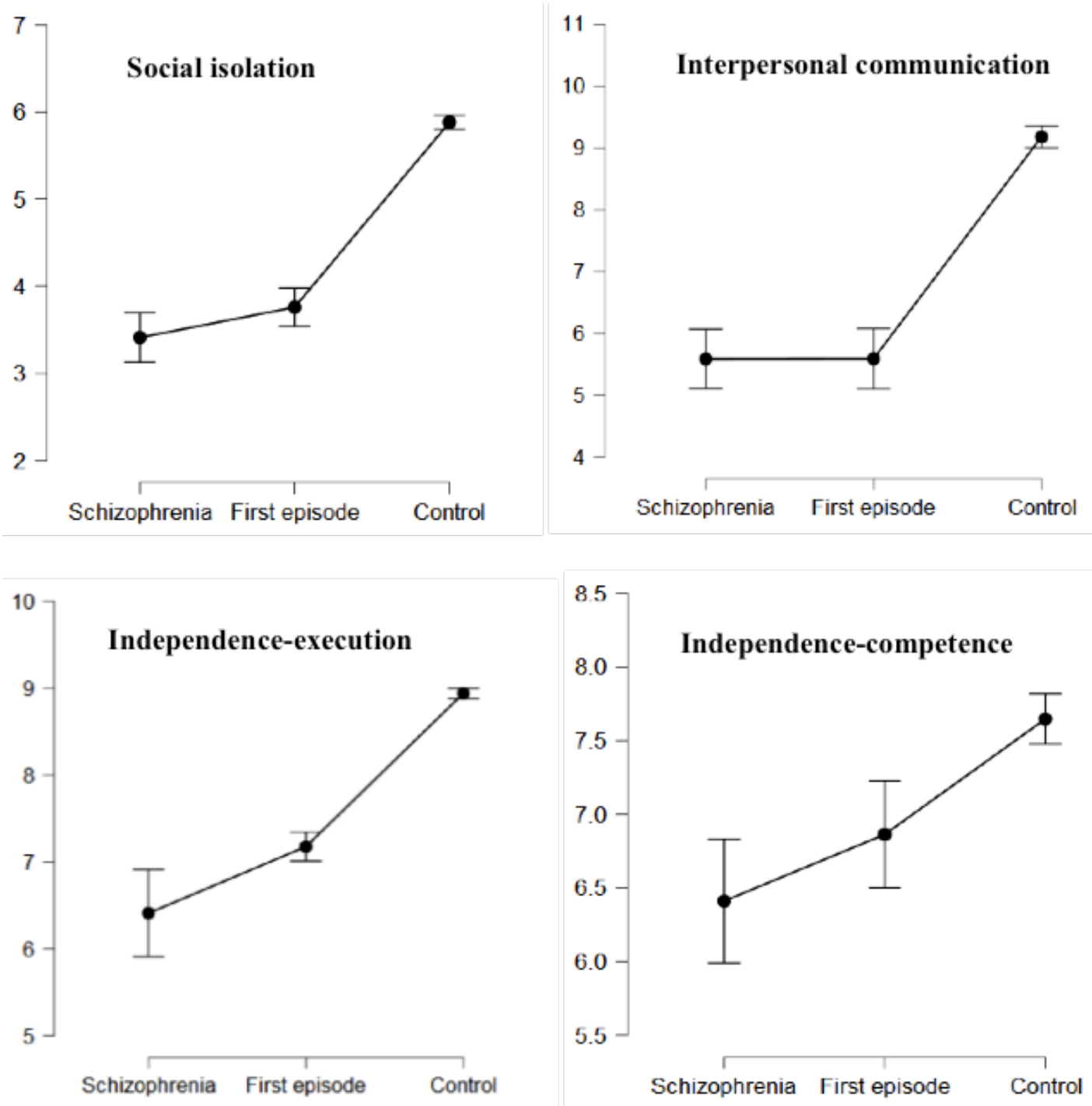

Figure 3. Comparisons between groups in social functioning areas. Bars represent standard errors.

\section{Mediation analysis}

Only the clinical participants that completed all assessment measures were included to carry out the mediation analysis $(n=23)$ (see Table 3 ). The mediation model: symptom severity (PANSS total) partially mediated the linkage between ToM and SA to SF, while neurocognition and parental interference were linked to SF regardless of symptoms (see Figure 4). The lower tables show the results of the mediation analysis: after testing whether indirect effects exist, total effects 
remained significant for all variables. The mediation model obtained excellent fit indexes: $\chi^{2}(2)=0.41 ; p=0.81 . \mathrm{CFI}=1.00 . \mathrm{TLI}=0.99 . \mathrm{GFI}=0.99 . \mathrm{SRMR}=0.025$; RMSN $=0.00(95 \% \mathrm{CI}=0.00-0.26)$. The model explained $67 \%$ of the variance in Global SF.

Table 3. Final linear regression model ${ }^{1}$

\begin{tabular}{llllllll}
\hline Predicted variable: Global Social Functioning & & & \\
& & & & & \multicolumn{2}{l}{$95 \%$ CI for $B$} & \\
\cline { 5 - 6 } Predictors & $B$ & $\beta$ & $p$-value & Lower & Upper & VIF \\
\hline Theory of Mind & 1.037 & 0.597 & 0.003 & 0.411 & 1.663 & 1.319 \\
Secure attachment & 0.198 & 0.459 & 0.013 & 0.047 & 0.348 & 1.242 \\
Neurocognition & 0.120 & 0.557 & 0,003 & 0.047 & 0.192 & 1.163 \\
Parental interference & 0.263 & 0.549 & 0.003 & 0.100 & 0.426 & 1.185 \\
\hline
\end{tabular}

Model fit. $R^{2}=0.60 . R M S E=3.59$. ANOVA: $F(4,18)=6.73 ; p=0.002$.

$B$. No standardised coefficients; $\beta$. Standardised coefficients; CI. Confidence Interval; VIF. Variance Inflation Factor.

${ }^{1}$ Only the final model is shown. Using the backward method, predictors that do not reach significance in each step are removed one by one until all remaining predictors are statistically significant $(p<0.05)$. 


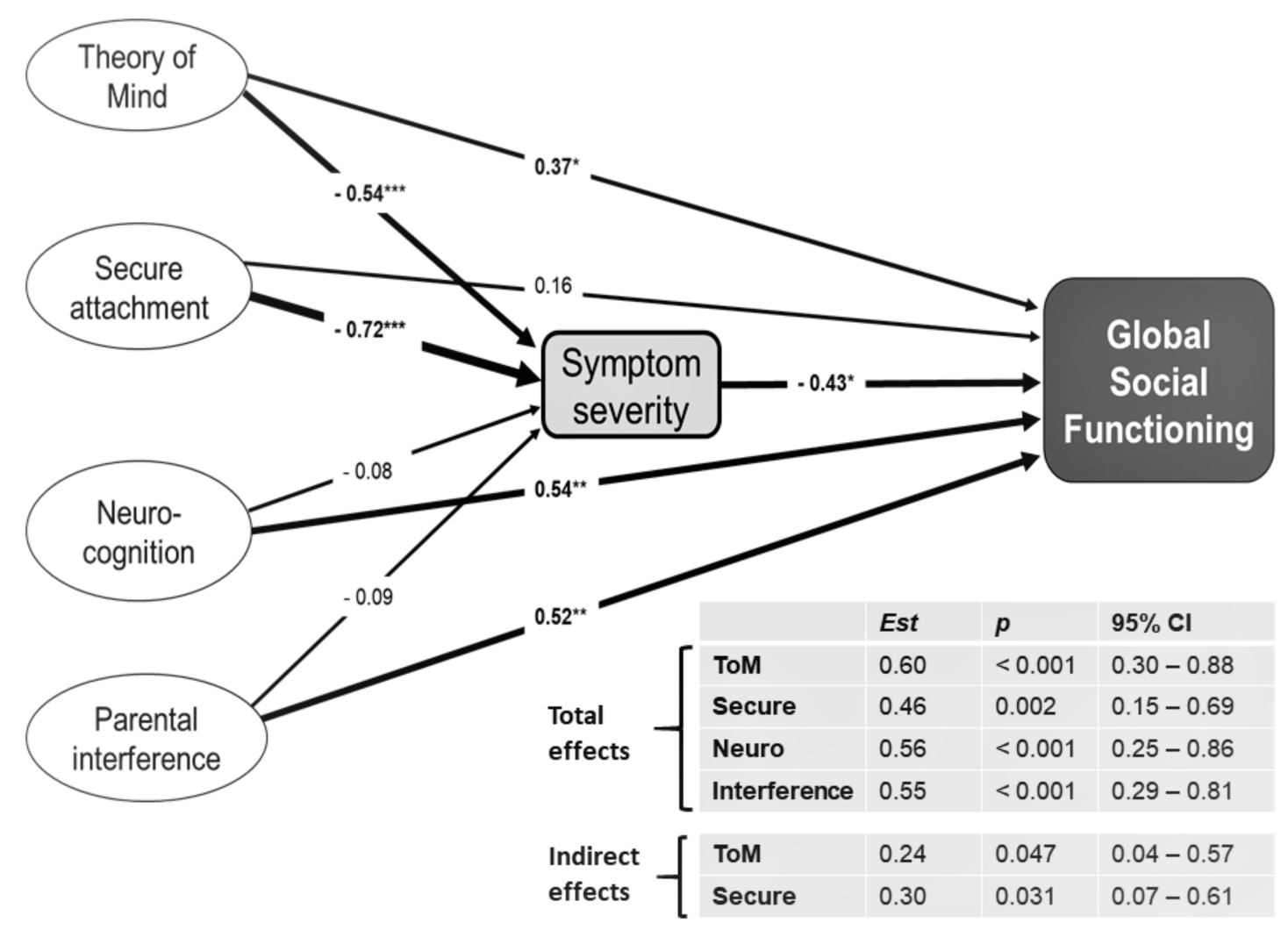

Figure 4. Mediation model. Symptom severity (PANSS total score) partially mediates the influence of Theory of Mind (ToM) and secure attachment on global social functioning (SF). Neurocognition and parental interference influence on SF regardless of symptoms. After testing whether indirect effects exist, total effects remained significant for all variables. The lower table shows indirect and total effects on SF. Est: Standardised coefficients. CI: Confidence Interval. ${ }^{*} p<0.05 ;{ }^{* *} p<0.01$; ${ }^{* *} p<0.001$. The model explained $67 \%$ of the variance in Global Social Functioning.

NOTE: Delta method standard errors, bias-corrected percentile bootstrap confidence intervals (1000 replications).

\section{Discussion}

Impairments in SF are frequently seen in schizophrenia (e.g. Lam et al., 2014). Differences between groups in all SF areas were found with large effect sizes, indicating relevant impairments in SF in both groups of persons with psychosis, as well as in neurocognition and social cognition domains. As expected, attachment differentiates between people with psychosis and healthy population, being more insecure in people with psychosis. However, contrary to previous literature, people with early psychosis have lower scores in child traumatism than stabilised psychosis and normal groups. Nevertheless, stabilised patients show more child traumatism than the normal population, according to 
the high rates of attachment disrupting events that individuals with psychosis tend to experience (Bailey et al., 2018). Exposure to traumatic life experiences significantly impacts on the pathogenesis of psychotic experiences and can lead to psychosis through many pathways that intersect with other genetic or environmental risk conditions (Popovic et al., 2019). This unexpected result could be explained because the small sample of participants with first episodes in which we assessed childhood trauma, should be insufficient to provide enough statistical power to show actual differences. Moreover, Lacasa \& Muela (2014) considered that the dimension of "child traumatism", provided by CAMIR-R, is not adequate to evaluate traumatic experiences. Therefore, CAMIR-R cannot evaluate the disorganised (fearful) style because it may involve lapses in monitoring of logical thinking or discourse.

Importantly, our results highlight a continuum in the severity of cognitive impairment from healthy people to stabilised psychotic persons, being less significant in individuals with early psychosis episodes than those with stabilised psychosis and differentiating clinical from healthy groups. These differences are more evident in neurocognition, draw to decide (beads task), emotion recognition, "optimistic" attributional style, and SF, but not regarding self-serving bias. However, attributional biases have been consistently found in schizophrenia (Monfort-Escrig \& Pena-Garijo, in press) and are considered as a risk factor for developing delusional believes (Moritz et al., 2018). Although the Kruskal-Wallis test indicated that differences between groups were not significant, a small but significant difference was found between the clinical and the healthy group (results not shown). Likewise, the observed continuum was not found in the case of ToM. Many studies report impairments in ToM across the psychosis continuum and phase of illness (Browne et al., 2016; Kamel \& AlQahtani, 2019; Ludwig et al., 2017). In the current work, we find significant differences between both clinical groups and the control one, but the first episode group obtained worse scores than the stabilised psychosis condition. This is an important issue, given that measuring ToM is essential for understanding and rehabilitating social cognitive 
impairments in persons with schizophrenia (Tan et al., 2018), especially in early psychosis (Mallawaarachchi et al., 2019).

Regarding predictors of SF, the relationship between neurocognition and social cognition with functional outcomes in schizophrenia is commonly accepted (Fett et al., 2011; Javed \& Charles, 2018; Kamel \& AlQahtani, 2019). In the current report, neurocognition predicts a better SF, regardless of symptom severity. Persons with psychosis often get low scores in neuropsychological tests (Jirsaraie et al., 2018) and there is consistent evidence for a significant relationship between the deficit syndrome and more severe neurocognitive impairment in schizophrenia (Bora et al., 2017). Despite the evidence, some authors note that neurocognitive deficits may be oversized, given that contextual factors due to symptoms during the assessment, may be masking the results (Beck et al., 2018). Unfortunately, this issue has not been considered sufficiently in most of the research (Pena-Garijo \& Monfort-Escrig, 2020a).

Social cognition is significantly impaired in individuals with schizophrenia and is a better predictor of poor functional outcomes than symptoms, or neurocognitive deficits are (Lam et al., 2014). The influence of ToM on SF appears to be partially mediated by symptoms. Our results suggest two routes: a direct path from ToM to SF, and an indirect one through symptom severity. Impairments in ToM have been broadly suggested as a predictor of psychotic symptoms (Brown et al., 2014; Rominger et al., 2016; Vaskinn et al., 2018) and have a stronger association with SF than other social cognition domains (Fett et al., 2011). Moreover, cognitive-behavioural interventions and others addressed to social cognition domains have demonstrated that changes in them could improve symptoms and SF in psychiatric patients (Horan \& Green, 2019; Javed \& Charles, 2018).

Finally, the influence of secure attachment on SF is partially mediated by symptom severity. Persons who experienced availability and support from their attachment figures tend to have fewer symptoms and, indirectly, a better SF. Furthermore, although people with a preoccupied attachment style may have 
maladaptive interpersonal patterns, their continued engagement in social relationships may act as a buffer against symptoms becoming clinically significant (Blair et al., 2018). This fact could explain the positive relationship that we find between parental interference (preoccupied attachment) and SF.

Our study presents some limitations. First, the small sample size does not allow to generalise the results easily. Moreover, most of the participants were male, and gender differences indicated that men were prone to have more symptoms. Unfortunately, separate analyses in the current dataset were not feasible. Second, the cross-sectional study design hinders the determination of causal relationships because it is impossible to know whether cognition or attachment experiences that patients relate preceded the onset of the symptoms and influenced SF outcomes or, on the contrary, the clinical condition modified the cognition and attachment-related memories. The methodology of the study, using mediation analyses, partially improves this limitation. Third, previous reports find cognitive biases and preoccupied attachment as mediators between childhood trauma and risk of psychosis (e.g., Gawęda et al., 2018). Although CAMIR-R provides a dimension called "child traumatism", it is not adequate to evaluate traumatic experiences (Lacasa \& Muela, 2014). Therefore, no "direct" measure of childhood trauma was available to include in our mediation model, which is another limitation. Finally, attachment, neurocognition and social cognition may account for an essential but limited part of real-life functioning in schizophrenia, and, therefore, these constructs need to be investigated more ecologically.

Future research should attempt to use better measures, larger and equal samples, comparisons with other psychopathological conditions, longitudinal designs, and a broader set of variables to improve these limitations.

\section{Conclusions}

Persons with psychotic disorders show substantial impairments in social functioning, neurocognition, and social cognition. Moreover, insecure attachment 
differentiates between clinical and healthy people. Our results highlight a continuum in the severity of impairments in cognition domains across the clinical conditions. Finally, neurocognition influences on social functioning, regardless of symptom severity, whereas psychotic symptoms partially mediate the influence of "theory of mind" and secure attachment. Results, although preliminary, suggest that cognition and attachment dimensions could be important targets to design therapeutic interventions to improve social functioning in persons with schizophrenia spectrum disorders, particularly in early psychosis.

\section{References}

Alonso, J., Olivares, J. M., Ciudad, A., Manresa, J. M., Casado, A., \& Gilaberte, I. (2008). Desarrollo y validación de la versión corta de la Escala de Funcionamiento Social en esquizofrenia para su uso en la práctica clínica. Actas Españolas de Psiquiatría, 36, 102-110.

American Psychiatric Association. (2014). Diagnostic and Statistical Manual of Mental Disorders (5th ed.). Arlington, VA: American Psychiatric Association.

Bailey, T., Alvarez-Jimenez, M., Garcia-Sanchez, A. M., Hulbert, C., Barlow, E., \& Bendall, S. (2018). Childhood Trauma Is Associated With Severity of Hallucinations and Delusions in Psychotic Disorders: A Systematic Review and Meta-Analysis. Schizophrenia Bulletin, 44,1111-1122. https://doi.org/10.1093/schbul/sbx161

Balluerka, N., Lacasa, F., Gorostiaga, A., \& Muela, A., 2011. CAMIR-R: Versión Reducida del cuestionario CAMIR. Psicothema, 23, 486-494. Retrieved from: http:// www.psicothema.com/psicothema.asp?id=3913

Beck, A. T., Himelstein, R., Bredemeier, K., Silverstein, S. M., \& Grant, P. (2018). What accounts for poor functioning in people with schizophrenia: A re-evaluation of the contributions of neurocognitive v. attitudinal and motivational factors. Psychological Medicine, 48, 2776-2785. https://doi.org/10.1017/S0033291718000442

Biesanz, J. C., Falk, C. F., \& Savalei, V. (2010). Assessing Mediational Models: Testing and Interval Estimation for Indirect Effects. Multivariate Behavioral Research, 45, 661701. https://doi.org/10.1080/00273171.2010.498292 
Blair, M. A., Nitzburg, G., DeRosse, P., \& Karlsgodt, K. H. (2018). Relationship between executive function, attachment style, and psychotic-like experiences in typically developing youth. Schizophrenia Research, 197, 428-433. https://doi.org/10.1016/ j.schres.2018.02.026

Bora, E., Binnur Akdede, B., \& Alptekin, K. (2017). Neurocognitive impairment in deficit and non-deficit schizophrenia: a meta-analysis. Psychological medicine, 47(14), 24012413. https://doi.org/10.1017/S0033291717000952

Brown, E. C., Tas, C., Can, H., Esen-Danaci, A., \& Brüne, M. (2014). A closer look at the relationship between the subdomains of social functioning, social cognition and symptomatology in clinically stable patients with schizophrenia. Comprehensive Psychiatry, 55, 25-32. https://doi.org/10.1016/j.comppsych.2013.10.001

Browne, J., Penn, D. L., Raykov, T., Pinkham, A. E., Kelsven, S., Buck, B., \& Harvey, P. D. (2016). Social cognition in schizophrenia: Factor structure of emotion processing and theory of mind. Psychiatry Research, 242, 150-156. https://doi.org/10.1016/ j.psychres.2016.05.034

Buck, B. E., Healey, K. M., Gagen, E. C., Roberts, D. L., \& Penn, D. L. (2016). Social cognition in schizophrenia: factor structure, clinical and functional correlates. Journal of Mental Health, 25, 330-337. https://doi.org/10.3109/09638237.2015.1124397

Candido, C. L., \& Romney, D. M. (1990). Attributional style in paranoid vs. depressed patients. British Journal of Medical Psychology, 63(4), 355-363. https://doi.org/ 10.1111/j.2044-8341.1990.tb01630.x

Corcoran, R., Mercer, G., \& Frith, C. D. (1995). Schizophrenia, symptomatology and social inference: Investigating "theory of mind" in people with schizophrenia. Schizophrenia Research, 17, 5-13. https://doi.org/10.1016/0920-9964(95)00024-G

Dudley, R. E. J., \& Over, D. E. (2003). People with delusions jump to conclusions: A theoretical account of research findings on the reasoning of people with delusions. Clinical Psychology and Psychotherapy, 10, 263-274. https://doi.org/10.1002/cpp.376

Fett, A. K. J., Viechtbauer, W., Dominguez, M. G., Penn, D. L., van Os, J., \& Krabbendam, L. (2011). The relationship between neurocognition and social cognition with functional outcomes in schizophrenia: A meta-analysis. Neuroscience \& Biobehavioral Reviews, 35, 573-588. https://doi.org/10.1016/J.NEUBIOREV.2010.07.001 
Garety, P., Freeman, D., Jolley, S., Ross, K., Waller, H., \& Dunn, G. (2011). Jumping to conclusions: The psychology of delusional reasoning. Advances in Psychiatric Treatment, 17, 332-339. https://doi.org/10.1192/apt.bp.109.007104

Gawęda, Ł., Pionke, R., Krężołek, M., Prochwicz, K., Kłosowska, J., Frydecka, D. ... Nelson, B. (2018). Self-disturbances, cognitive biases and insecure attachment as mechanisms of the relationship between traumatic life events and psychotic-like experiences in non-clinical adults - A path analysis. Psychiatry Research, 259, 571578. https://doi.org/10.1016/j.psychres.2017.11.009

Gil, D., Fernández-Modamio, M., Bengochea, R., \& Arrieta, M. (2012). Adaptación al español de la prueba de teoría de la mente Hinting Task. Revista de Psiquiatría y Salud Mental, 5, 79-88. https://doi.org/10.1016/j.rpsm.2011.11.004

Gil-Sanz, D., Fernández-Modamio, M., Bengochea-Seco, R., Arrieta-Rodríguez, M., González-Fraile, E., Pérez-Fuentes, G. ... Santos-Zorrozúa, B. (2017). PERE: Una nueva herramienta para valorar el reconocimiento de las emociones básicas y su aplicación en la esquizofrenia. Revista de Psicopatología y Psicología Clínica, 22, 85-93. https:// doi.org/10.5944/rppc.vol.22.num.2.2017.17244

Horan, W. P., \& Green, M. F. (2019). Treatment of social cognition in schizophrenia: Current status and future directions. Schizophrenia Research, 203, 3-11. https:// doi.org/10.1016/j.schres.2017.07.013

Hu, L. T., \& Bentler, P. M. (1999). Cutoff criteria for fit indexes in covariance structure analysis: Conventional criteria versus new alternatives. Structural Equation Modeling, 6, 1-55. https://doi.org/10.1080/10705519909540118

JASP Team (2020). JASP (Version 0.13.1) [Computer software]. https://jasp-stats.org/

Javed, A., \& Charles, A. (2018). The importance of social cognition in improving functional outcomes in schizophrenia. Frontiers in Psychiatry, 9, 1-14. https:// doi.org/10.3389/fpsyt.2018.00157

Jirsaraie, R. J., Sheffield, J. M., \& Barch, D. M. (2018). Neural correlates of global and specific cognitive deficits in schizophrenia. Schizophrenia Research, 201, 237-242. https://doi.org/10.1016/j.schres.2018.06.017

Kamel, N., \& AlQahtani, F. (2019). Social Cognition in Schizophrenia: A Review Study. Open Journal of Psychiatry, 9(2), 81-97. https://doi.org/10.4236/ojpsych. 2019.92007 
Kay, S. R., Fiszbein, A., \& Opler, L. A. (1987). The positive and negative syndrome scale (PANSS) for schizophrenia. Schizophrenia Bulletin, 13, 261-276. https://doi.org/ $10.1093 /$ schbul/13.2.261

Korver-Nieberg, N., Berry, K., Meijer, C. J., \& De Haan, L. (2014). Adult attachment and psychotic phenomenology in clinical and non-clinical samples: A systematic review. Psychology and Psychotherapy:Theory, Research and Practice, 87, 127-154. https:// doi.org/10.1111/papt.12010

Lacasa, F., \& Muela, A. (2014). Guía para la aplicación e interpretación del cuestionario de apego CaMir-R. Psicopatología y Salud Mental, 24, 83-93.

Lam, B. Y., Raine, A., \& Lee, T. M. (2014). The relationship between neurocognition and symptomatology in people with schizophrenia: Social cognition as the mediator. $B M C$ Psychiatry, 14, 138. https://doi.org/10.1186/1471-244X-14-138

Ludwig, K. A., Pinkham, A. E., Harvey, P. D., Kelsven, S., \& Penn, D. L. (2017). Social cognition psychometric evaluation (SCOPE) in people with early psychosis: A preliminary study. Schizophrenia Research, 190, 136-143. https://doi.org/10.1016/ j.schres.2017.03.001

Mallawaarachchi, S. R., Cotton, S. M., Anderson, J., Killackey, E., \& Allott, K. A. (2019). Exploring the use of the Hinting Task in first-episode psychosis. Cognitive Neuropsychiatry, 24, 65-79. https://doi.org/10.1080/13546805.2019.1568864

Monfort-Escrig, C., \& Pena-Garijo, J. (in press). Attributional styles and Social Functioning in Schizophrenia. Is the Learned Helplessness model suitable? Clínica y Salud.

Moritz, S., Bentall, R. P., Kolbeck, K., \& Roesch-Ely, D. (2018). Monocausal attribution and its relationship with reasoning biases in schizophrenia. Schizophrenia Research, 193, 77-82. https://doi.org/10.1016/j.schres.2017.06.057

Pena-Garijo, J., \& Monfort-Escrig, C. (2020a). Cognition in schizophrenia. State of the art (Part I): assessment methods and neural correlates. Revista Asociación Española de Neuropsiquiatría, 40, 109-130. Retrieved from: http://www.revistaaen.es/index.php/ aen/article/view/17121

Pena-Garijo, J., \& Monfort-Escrig, C. (2020b). Cognition in schizophrenia. State of the art (Part II): cognitive biases, explanatory models, and intervention programs. Revista 
Asociación Española de Neuropsiquiatría, 40, 131-154. Retrieved from: http:// www.revistaaen.es/index.php/aen/article/view/17122

Peralta, V., \& Cuesta, M. J. (1994). Psychometric properties of the Positive and Negative Syndrome Scale (PANSS) in schizophrenia. Psychiatry Research, 53, 31-40. https:// doi.org/10.1016/0165-1781(94)90093-0

Peterson, C., Semmel, A., von Baeyer, C., Abramson, L. Y., Metalsky, G. I., \& Seligman, M. E. P. (1982). The attributional Style Questionnaire. Cognitive Therapy and Research, 6, 287-299. https://doi.org/10.1007/BF01173577

Pierrehumbert, B., Karmaniola, A., Sieye, A., Meister, C., Miljkovitch, R., \& Halfon, 0. (1996). Les modeles de relations: Developpement d'un autoquestionnaire d'attachement pour adultes. Psychiatrie de l'Enfant, 39, 161-206.

Pino, O., Guilera, G., Rojo, J. E., Gómez-Benito, J., Bernardo, M., Crespo-Facorro, B. ... Rejas, J. (2008). Spanish version of the Screen for Cognitive Impairment in Psychiatry (SCIPS): Psychometric properties of a brief scale for cognitive evaluation in schizophrenia. Schizophrenia Research, 99, 139-148. https://doi.org/10.1016/j.schres.2007.09.012

Popovic, D., Schmitt, A., Kaurani, L., Senner, F., Papiol, S., Malchow, B. ... Falkai, P. (2019). Childhood Trauma in Schizophrenia: Current Findings and Research Perspectives. Frontiers in Neuroscience, 13, 274. https://doi.org/10.3389/fnins.2019.00274

Rokita, K. I., Dauvermann, M. R., \& Donohoe, G. (2018). Early life experiences and social cognition in major psychiatric disorders: A systematic review. European Psychiatry, 53, 123-133. https://doi.org/10.1016/j.eurpsy.2018.06.006

Rominger, C., Bleier, A., Fitz, W., Marksteiner, J., Fink, A., Papousek, I., \& Weiss, E. M. (2016). Auditory top-down control and affective theory of mind in schizophrenia with and without hallucinations. Schizophrenia Research, 174, 192-196. https:// doi.org/10.1016/J.SCHRES.2016.05.006

Rosseel, Y. (2012). lavaan: An R Package for Structural Equation Modeling. Journal of Statistical Software, 48(2), 1-36. http://dx.doi.org/10.18637/jss.v048.i02

Sanjuán, P., Fraguas, D., Magallares, A., \& Merchán-Naranjo, J. (2009). Depressive symptomatology and attributional style in patients with schizophrenia. Clinical Schizophrenia and Related Psychoses, 3, 31-38. https://doi.org/10.3371/CSRP.3.1.3

Sanjuán, P., Magallares, A., González, J. L., \& Pérez-García, A. (2013). A study on the validity of the Spanish version of the Attributional Style Questionnaire for negative 
situations. Revista de Psicopatologia y Psicologia Clinica, 18(1), 61-71. https:// doi.org/10.5944/rppc.vol.18.num.1.2013.12763

Sheinbaum, T., Bedoya, E., Ros-Morente, A., Kwapil, T. R., \& Barrantes-Vidal, N. (2013). Association between attachment prototypes and schizotypy dimensions in two independent non-clinical samples of Spanish and American young adults. Psychiatry Research, 210, 408-413. https://doi.org/10.1016/j.psychres.2013.07.020

Tan, B. L., Lee, S. A., \& Lee, J. (2018). Social cognitive interventions for people with schizophrenia: A systematic review. Asian Journal of Psychiatry, 35, 115-131. https:// doi.org/10.1016/j.ajp.2016.06.013

Torres, A., \& Olivares, J. M. (2005). Validation of the Spanish version of the social functioning scale. Actas Espanolas de Psiquiatria, 33(4), 216-220.

Vaskinn, A., Andersson, S., Østefjells, T., Andreassen, O. A., \& Sundet, K. (2018). Emotion perception, non-social cognition and symptoms as predictors of theory of mind in schizophrenia. Comprehensive Psychiatry, 85, 1-7. https://doi.org/10.1016/ j.comppsych.2018.05.002 


\section{Discusión general}

\section{Principales hallazgos}

Los resultados sobre los estilos atribucionales proporcionan evidencia sobre la utilidad y la adecuación del Modelo reformulado de la Indefensión Aprendida (Abramson et al., 1978) y su forma más articulada, la Teoría de la Depresión por Deseperanza (Davidson, Lesser, Parente y Fiszdon, 2018; Haeffel et al., 2017), para predecir depresión en personas con trastornos del espectro de la esquizofrenia. Los resultados se mantuvieron significativos después de calcular correlaciones parciales con el fin de controlar los síntomas psicóticos. Siendo este un hallazgo importante que apoya la hipótesis sobre la idoneidad del Modelo de la Indefensión Aprendida para predecir la depresión en personas con trastornos del espectro de la esquizofrenia, independientemente de los síntomas psicóticos.

Las personas con trastornos del espectro de la esquizofrenia, en comparación con un grupo de población no clínica, mostraron un mayor sesgo de atribución negativo en concordancia con nuestras hipótesis. Además, estas personas muestran un sesgo de atribución "pesimista" como un rasgo diferencial importante en comparación al grupo control, con un gran tamaño del efecto. Por otro lado, nuestros resultados demuestran la hipótesis de que las personas con trastornos del espectro de la esquizofrenia tienen un estilo de atribución "depresivo"; no obstante, no se encontraron diferencias en la atribución interna para situaciones negativas. Los resultados muestran una tendencia a ver los eventos de la vida como el resultado de causas inestables o impredecibles (sesgo de atribución inestable) y no un sesgo de externalización de eventos negativos, lo que predice mejor la gravedad de los síntomas positivos, según nuestra hipótesis y de acuerdo con los hallazgos de Lysaker, Lancaster, Nees y Davis (2004). La creencia de que las causas de situaciones positivas y negativas son generalizables y se relacionan con muchas áreas de la vida (estilo de atribución global) predice 
un mejor funcionamiento social general y una mejor comunicación interpersonal en las personas con trastornos del espectro de la esquizofrenia. Sin embargo, en contraste con Lysaker et al., (2004), los síntomas negativos no se relacionan con un peor funcionamiento social, aunque un sesgo de atribución inestable se relaciona significativamente con el predominio de síntomas negativos. Puntuaciones altas en psicopatología general también predicen una menor independencia para las actividades de la vida diaria. Un mayor nivel educativo, una mejor situación laboral y una atribución estable de situaciones positivas se relacionan con un menor aislamiento social. Las personas con trastornos psicóticos que creen que los eventos positivos de su vida son estables y predecibles, disfrutan de mejores relaciones sociales y están menos aisladas según Lysaker et al., (2004), lo que sugiere que el estilo de atribución estable es el más relacionado con las diferentes medidas del funcionamiento social. Finalmente, las personas con trastornos del espectro de la esquizofrenia que muestran un estilo atribucional positivo (atribución interna, estable y global) tienen más probabilidades de permanecer activas.

El objetivo principal del estudio de las dimensiones del apego se enfocaba a probar la adecuación de la medida CAMIR-R para evaluar patrones de apego en muestra clínica y no clínica. El CAMIR-R obtuvo buenos coeficientes de consistencia interna, por lo que se mostró fiable para su uso en muestras de personas con trastornos del espectro de la esquizofrenia. Como sugerían nuestras hipótesis, se encontraron diferencias entre personas con psicosis y población no clínica, siendo el apego más inseguro en personas con psicosis. Los resultados muestran un mayor apego inseguro en el grupo clínico con mayor porcentaje de estilo evitativo, en concordancia con revisiones recientes (Gumley, Taylor, Schwannauer y MacBeth, 2014; Korver-Nieberg, Berry, Meijer y De Haan, 2014; Carr, Hardy y Fornells-Ambrojo, 2018). Los síntomas negativos de la esquizofrenia a menudo se presentan como tipos de comportamiento de "evitación" y dan como resultado una falta de relaciones interpersonales significativas (Crowell, 2014). 
Además, se observó que las dimensiones del apego predicen varias áreas del funcionamiento social y de manera independiente a los síntomas psicóticos. Se encontraron diferencias entre los grupos en todas las áreas del funcionamiento social con grandes tamaños del efecto, lo que indica déficits importantes en el funcionamiento social en personas con psicosis. El apego seguro correlacionó positivamente con todas las áreas del funcionamiento social, considerando la muestra completa. La preocupación familiar (apego preocupado), la autosuficiencia (apego evitativo) y el traumatismo infantil se relacionan con un peor funcionamiento social. Nuestros resultados son consistentes con hallazgos recientes que demuestran cómo las predicciones derivadas de la teoría del apego juegan un papel en el flujo natural de la vida real (Sheinbaum et al., 2015).

La psicopatología general predijo un peor funcionamiento social general, mientras que la interferencia de los padres predijo mejores resultados. Aunque las personas con un estilo de apego preocupado pueden tener patrones interpersonales desadaptativos, su participación continua en las relaciones sociales puede actuar como un amortiguador contra los síntomas que se vuelven clínicamente significativos (Blair, Nitzburg, DeRosse y Karlsgodt, 2018) y, debido a las características de la muestra (la mayoría pacientes estables de largo plazo), podríamos suponer que las relaciones familiares podrían proporcionar suficiente apoyo social. Los hallazgos también demuestran que las personas que experimentaron un apego preocupado tienden a tener niveles más altos de aislamiento social (Korver-Nieberg, Berry, Meijer y DeHaan, 2014). Encontramos que los síntomas negativos predicen una peor comunicación interpersonal y menores niveles de independencia-competencia en la vida diaria, según estudios previos (Lacasa y Muela, 2014).

Según nuestra hipótesis, los análisis confirman que varias dimensiones del apego están vinculadas al funcionamiento social, independientemente de los síntomas. Los modelos detallados para áreas específicas del funcionamiento social muestran cómo la preocupación familiar está relacionada con el aislamiento social y la situación laboral, independientemente de los síntomas. 
Asimismo, la interferencia de los padres está ligada a la comunicación interpersonal y la permisividad de los padres con el nivel educativo. A pesar de su papel potencial como predictor del funcionamiento social, el apego solo representa una parte limitada en el funcionamiento de la vida real y, por lo tanto, se necesita más investigación para aclarar este problema. Estudios anteriores indican que la interacción entre los sesgos cognitivos, las autoperturbaciones y el apego inseguro puede ser de gran importancia en el inicio de las experiencias psicóticas (Sheinbaum et al., 2015).

Los resultados sobre la centralidad del apego seguro dentro de la red interactiva, están en la línea de nuestra hipótesis, donde el apego seguro aparece como el rol central dentro de la red de interacción, seguido por la psicopatología general. La red interactiva muestra cómo los nodos que pertenecen a cada construcción, generalmente, se presentan fuertemente asociados y próximos entre sí, a excepción de la ToM. En general, observamos que: el apego seguro y la ToM se correlacionan negativamente con la psicopatología general; la preocupación familiar (apego preocupado) se relaciona positivamente con los síntomas negativos e inversamente con la neurocognición; la neurocognición se correlaciona inversamente con los síntomas negativos; y el apego seguro y la ToM están inversamente correlacionados. Esta última correlación supone un resultado inesperado; entendemos que esta relación puede explicarse por las características de la muestra, que incluye pacientes con primer episodio y pacientes crónicos. Es probable que la relación entre el apego y la ToM cambie a lo largo de la vida, en particular entre la adolescencia y la edad adulta (Hünefeldt et al., 2013).

Nuestros resultados concuerdan con la hipótesis planteada e indican que la influencia del apego seguro sobre el funcionamiento social está mediada por la gravedad de los síntomas. Las personas que experimentaron disponibilidad y apoyo de sus figuras de apego tienden a tener menos síntomas e, indirectamente, un mejor funcionamiento social. Además, aunque las personas con un estilo de 
apego preocupado pueden tener patrones interpersonales desadaptativos, su compromiso continuo en las relaciones sociales puede actuar como un amortiguador contra los síntomas que se vuelven clínicamente significativos (Blair et al., 2018). Este hecho podría explicar la correlación positiva que encontramos entre la interferencia de los padres (apego preocupado) y el funcionamiento social.

Los déficits en el funcionamiento social se observan con frecuencia en la esquizofrenia en relación con la neurocognición y la cognición social (Fett et al., 2011; Javed y Charles, 2018; Kamel y AlQahtani, 2019; Lam et al., 2014). Nuestros resultados indican que la neurocognición predice un mejor funcionamiento social, independientemente de la gravedad de los síntomas. Las personas con psicosis suelen obtener puntuaciones bajas en las pruebas neuropsicológicas (Aleman, 2014; Jirsaraie et al., 2018; Wojciechowska et al., 2016). A pesar de la evidencia, algunos autores señalan que los déficits neurocognitivos pueden estar sobredimensionados, dado que factores contextuales como la motivación, la ansiedad o la distracción por síntomas durante la evaluación, pueden estar enmascarando los resultados (Beck et al., 2018; Moritz et al., 2017).

Por último, la influencia de la ToM en el funcionamiento social parece estar parcialmente mediada por los síntomas. Nuestros resultados sugieren dos rutas: una ruta directa de la ToM al funcionamiento social, y una ruta indirecta a través de la gravedad de los síntomas. Los déficits en ToM se han sugerido ampliamente como un predictor de síntomas psicóticos (Brown et al., 2014; Fretland et al., 2015; Rominger et al., 2016; Vaskinn et al., 2018) y tienen una asociación más fuerte con el funcionamiento social que otros dominios de la cognición social (Fett et al., 2011). Además, la ToM también se ve afectada a lo largo del continuo de la psicosis y la fase de la enfermedad (Browne et al., 2016; Green et al., 2015; Kamel y AlQahtani, 2019; Ludwig et al., 2017; Mallawaarachchi et al., 2019; Pinkham et al., 2017). 
Los resultados sobre los déficits en el funcionamiento social en personas con trastornos del espectro de la esquizofrenia, muestran diferencias entre los grupos en todas las áreas del funcionamiento social con grandes tamaños del efecto. En concordancia con nuestras hipótesis, esto indica la existencia de déficits relevantes en el funcionamiento social en personas con psicosis, así como en los dominios de la neurocognición y la cognición social.

Como se ha observado en los estudios anteriores, el apego diferencia entre personas con psicosis y población no clínica, siendo más inseguro en personas con psicosis. Sin embargo, contrariamente a la literatura, las personas con psicosis temprana muestran puntuaciones más bajas en traumatismo infantil que en personas con psicosis estable y del grupo no clínico. Este resultado inesperado podría explicarse porque la pequeña muestra de participantes con primeros episodios, podría ser insuficiente para proporcionar adecuado poder estadístico para mostrar las diferencias actuales. No obstante, la dimensión de "traumatismo infantil" del CAMIR-R no es adecuada para evaluar las experiencias traumática (Lacasa y Muela, 2014), por lo que no se puede evaluar el estilo desorganizado (temeroso) al poder implicar lapsos en el seguimiento del pensamiento lógico o del discurso.

Es importante destacar que nuestros resultados muestran un continuo en la gravedad del deterioro cognitivo de personas sanas a personas psicóticas estables, siendo menos significativo en individuos con episodios de psicosis temprana que en aquellos con psicosis estable y, diferenciando los grupos clínicos del no clínico. Estas diferencias son más evidentes en la neurocognición, extraer para decidir (beads task), reconocimiento de emociones, estilo atribucional "optimista" y funcionamiento social, pero no en lo que respecta al sesgo personalizador. No obstante, en la esquizofrenia se han encontrado sesgos atribucionales y se consideran un factor de riesgo para el desarrollo de las creencias delirantes. Por otro lado, en el continuo no se observó la ToM aunque la literatura informa de déficits en la ToM a lo largo del continuo de la psicosis y la fase de la enfermedad (Browne et al. 2016; Kamel y AlQahtani, 2019; Ludwig et al., 2017). En el trabajo actual encontramos diferencias significativas entre ambos 
grupos clínicos y el grupo control, pero el grupo de pacientes con primer episodio obtuvo peores puntuaciones en comparación al grupo de pacientes con psicosis estable.

En relación a los predictores del funcionamiento social, como se ha mencionado en el estudio anterior, la neurocognición predijo un mejor funcionamiento social, independientemente de la gravedad de los síntomas. Por otro lado, la influencia de la ToM en el funcionamiento social parece estar parcialmente mediada por los síntomas y se sugieren dos rutas explicativas, mencionadas anteriormente. Finalmente, la influencia del apego seguro en el funcionamiento social está, igualmente, mediada parcialmente por la gravedad de los síntomas.

\section{Fortalezas}

La investigación aquí presentada, ofrece una serie de fortalezas que consideramos importantes señalar. En primer lugar, respecto a los estilos atribucionales, nuestros resultados actualizan los estudios clásicos basados en la teoría de la atribución del Modelo de Indefensión Aprendida. En este sentido, nuestro estudio aporta evidencia de que el Modelo reformulado de Indefensión Aprendida proporciona utilidad para predecir la depresión en personas con trastornos del espectro de la esquizofrenia, independientemente de los síntomas psicóticos. El mecanismo subyacente parece implicar un sesgo "pesimista" hacia la interpretación de los acontecimientos de la vida.

En segundo lugar, hasta donde sabemos, este es el primer estudio que utiliza CAMIR para evaluar los estilos de apego en personas con trastornos psicóticos. El instrumento demostró idoneidad para su uso en muestras psicóticas, mostrando índices de consistencia interna aceptables. La metodología del estudio, utilizando análisis de mediación, permite concluir que varias dimensiones del apego influyen en el funcionamiento social, independientemente de la gravedad de los síntomas. 
En tercer término, esta puede ser también la primera investigación que aborda simultáneamente el análisis de redes y de mediación. En primer lugar, para identificar el papel más o menos central que los síntomas psicóticos, la cognición y las dimensiones del apego podrían desempeñar dentro de una red interactiva para explicar la fenomenología psicótica. Y, a continuación, explorar si la gravedad de los síntomas media la influencia de las variables seleccionadas para predecir el funcionamiento social en los trastornos del espectro de la esquizofrenia. Los enfoques de red ofrecen alternativas metodológicas prometedoras a las estrategias de investigación tradicionales, que generalmente se basan en la idea de que los síntomas son manifestaciones de algún factor subyacente común. Además, estas técnicas pueden ofrecer posibilidades para guiar y evaluar intervenciones terapéuticas (Borsboom y Cramer, 2013).

Nuestros estudios proporcionan evidencia preliminar sobre una estructura de red en la que el apego seguro y la psicopatología general son los nodos más centrales dentro de la red interactiva de síntomas, cognición y dimensiones de apego en los trastornos del espectro de la esquizofrenia. Además, la neurocognición tiene un fuerte efecto directo sobre el funcionamiento social, mientras que los síntomas psicóticos median parcialmente los efectos del apego seguro y la ToM. Finalmente, la inclusión de algunos procedimientos nuevos para evaluar la fiabilidad de los modelos propuestos agrega un plus de bondad a nuestro estudio y mejora parcialmente las limitaciones debido al pequeño tamaño de la muestra. Más allá de la importancia de los síntomas clínicos, nuestros resultados, aunque preliminares, sugieren la necesidad de evaluar las experiencias de apego, las deficiencias cognitivas y los resultados funcionales para mejorar las intervenciones específicas para promover la recuperación en personas con trastornos del espectro esquizofrénico.

\section{Limitaciones}

Nuestra investigación presenta algunas limitaciones. En primer lugar, el pequeño tamaño de la muestra no permite generalizar los resultados fácilmente. 
El diseño de estudio transversal dificulta la determinación de las relaciones causales, ya que no es posible saber si las experiencias cognitivas, las experiencias de apego y los estilos de atribución que relatan los pacientes precedieron al inicio de los síntomas e influyeron en los resultados del funcionamiento social o, por el contrario, los síntomas modificaron las variables en el momento presente. En los estudios que se incluyó el análisis de mediación esta limitación mejoró parcialmente. La mayoría de los participantes eran hombres y las diferencias de género indicaron que estos eran propensos a tener más síntomas; no obstante, no fue posible realizar análisis separados en el conjunto de datos actual.

Los resultados de investigaciones anteriores encontraron los sesgos cognitivos y el apego preocupado como mediadores entre el trauma infantil y el riesgo de psicosis (por ejemplo, Gaweda et al., 2018). Si bien el CAMIR-R aporta una dimensión denominada "traumatismo infantil" no es adecuada para evaluar las experiencias traumáticas (Lacasa y Muela, 2014). Por lo tanto, no se dispuso de una medida "directa" del trauma infantil para incluir en el modelo de mediación Dada la novedad de los modelos de redes en psicopatología, se requieren métodos rigurosos para evaluar la fiabilidad de las redes estimadas. En este sentido, se utilizaron algunos procedimientos recomendados por Epskamp et al. (2018). Nuestros resultados indicaron que la red generada debe interpretarse con cautela debido a los amplios intervalos de confianza y de la inestabilidad de algunas medidas de centralidad. Por último, el apego, la neurocognición y la cognición social pueden explicar una parte esencial pero limitada del funcionamiento de la vida real en la esquizofrenia y, por lo tanto, estos constructos deben investigarse de manera más ecológica.

\section{Perspectivas de futuro}

Las investigaciones futuras deberían estar basadas en estudios de diseño longitudinal con muestras más apropiadas con el fin de mejorar algunas de las limitaciones mencionadas, así como la utilización de métodos de evaluación más 
ecológicos para evitar las deficiencias que muestran algunos autoinformes. El apego, la neurocognición y la cognición social pueden explicar una parte esencial pero limitada del funcionamiento de la vida real en la esquizofrenia y, por lo tanto, estos constructos deben investigarse de manera más ecológica. La investigación futura debe intentar utilizar mejores medidas, incluir métodos rigurosos para evaluar la fiabilidad de los modelos, muestras más grandes e iguales, comparaciones con otras condiciones psicopatológicas, diseños longitudinales y un conjunto más amplio de variables para mejorar las limitaciones. 


\section{Conclusiones}

- El modelo reformulado de la indefensión aprendida proporciona utilidad para predecir la depresión en personas con trastornos del espectro de la esquizofrenia, independientemente de los síntomas psicóticos. El mecanismo parece implicar un sesgo "pesimista" hacia la interpretación de los acontecimientos de la vida.

- Un sesgo de atribución inestable (pero no externo) resulta más predictivo para explicar los síntomas positivos, y un estilo de atribución global predice un mejor funcionamiento social.

- Por lo que sabemos, este es el primer estudio que utiliza el CAMIR para evaluar estilos de apego en personas con trastornos psicóticos. El instrumento demostró ser adecuado para su uso en muestras de personas con psicosis, mostrando índices de consistencia interna aceptables.

- Las personas con psicosis muestran un patrón de apego inseguro con una mayor prevalencia del estilo evitativo.

- La psicopatología general, los síntomas negativos y algunas dimensiones del apego predicen un peor funcionamiento social.

- Las personas con psicosis que recuerdan haber tenido límites adecuados durante la infancia tienden a ser más competentes y autónomos en sus actividades de la vida diaria y alcanzan un mejor nivel educativo.

- Varias dimensiones del apego influyen en el funcionamiento social, independientemente de la gravedad de los síntomas.

- Recientemente, la conceptualización del sistema dinámico de psicopatología ha ganado terreno, lo que ha llevado al desarrollo de "modelos de red". Este estudio proporciona evidencia preliminar sobre una estructura de red en la que el apego seguro y la psicopatología general son los nodos más centrales 
dentro de una red de síntomas, cognición y dimensiones de apego en la psicosis.

- Las personas con trastornos psicóticos muestran deficiencias sustanciales en el funcionamiento social, la neurocognición y la cognición social. Nuestros resultados ponen de relieve un continuum de gravedad en los déficits a través de diferentes condiciones clínicas.

- Finalmente, la neurocognición influye sobre el funcionamiento social, independientemente de la gravedad de los síntomas, mientras que los síntomas psicóticos median parcialmente la influencia de la "teoría de la mente" y del apego seguro.

Los resultados expuestos en la presente tesis, aunque preliminares, sugieren que las dimensiones de la cognición y el apego podrían ser objetivos sustanciales para diseñar intervenciones terapéuticas para mejorar el funcionamiento social en personas con trastornos del espectro de esquizofrenia. 


\section{Referencias bibliográficas}

Abramson, L. Y., Seligman, M. E. P. y Teasdale, J. (1978). Learned helplessness in humans: Critique and reformulation. Journal of Abnormal Psychology, 87, 49-74.

Achim, A. M. y Lepage, M. (2005). Episodic memory-related activation in schizophrenia: Meta-analysis. The British Journal of Psychiatry, 187(6), 500-509. https://doi.org/ 10.1192/bjp.187.6.500

Adolphs, R. (2009). The social brain: neural basis of social knowledge. Annual Review Psychology, 60:693-716. https://dx.doi.org/10.1146\%2Fannurev.psych. 60.110707.163514

Acosta, F. J., Hernández, J. L., Pereira, J., Herrera, J. y Rodríguez, C. J. (2012). Medication adherence in schizophrenia. World journal of psychiatry, 2(5), 74-82. https://doi.org/ 10.5498/wjp.v2.i5.74

Ainsworth, M. S. (1989). Attachments beyond infancy. American Psychologist, 44(4), 709-716. https://doi.org/10.1037/0003-066X.44.4.709

Aleman, A. (2014). Neurocognitive Basis of Schizophrenia: Information Processing Abnormalities and Clues for Treatment. Advances in Neuroscience, 2014, 104920. http://doi.org/10.1155/2014/104920

Alonso, J., Olivares, J. M., Ciudad, A., Manresa, J. M., Casado, A. y Gilaberte, I. (2008). Desarrollo y validación de la versión corta de la Escala de Funcionamiento Social en esquizofrenia para su uso en la práctica clínica. Actas Españolas de Psiquiatría, 36, 102-110.

Alonso-Arbiol, I., Balluerka, N. y Shaver, P. R. (2007). A Spanish version of the Experiences in Close Relationships (ECR) adult attachment questionnaire. Personal Relationships, 14(1), 45-63. https://doi.org/10.1111/j.1475-6811.2006.00141.x

American Psychiatric Association (2000). Diagnostic and Statistical Manual of Mental Disorders (4rth ed., Text Revision). Washington, DC: American Psychiatric Association. American Psychiatric Association (2013). Diagnostic and Statistical Manual of Mental Disorders (5th ed.). Arlington, VA: American Psychiatric Association. 
American Psychiatric Association (2021). Practice Guideline for the Treatment of Patients With Schizophrenia (3rd ed.). Washington, DC: American Psychiatric Association.

Andreasen N. C. (1984a). Scale for the Assessment of Negative Symptoms. Iowa City: University of Iowa.

Adreasen, N. C. (1984b). Scale for the Assessment of Positive Symptoms. Iowa City: University of Iowa.

Andreou, C., Treszl, A., Roesch-Ely, D., Köther, U., Veckenstedt, R. y Moritz, S. (2014). Investigation of the role of the jumping-to-conclusions bias for short-term functional outcome in schizophrenia. Psychiatry Research, 218(3), 341-347. https://doi.org/ 10.1016/j.psychres.2014.04.040

Aydin, O., Balikci, K., Tas, C., Aydin, P. U., Danaci, A. E., Brüne, M. y Lysaker, P. H. (2016). The developmental origins of metacognitive deficits in schizophrenia. Psychiatry research, 245, 15-21. https://doi.org/10.1016/j.psychres.2016.08.012

Aydin, O., Balikçi, K., Tas, C., Ünal-Aydin, P., Taneli, F. y Esen-Danaci, A. (2019). Assessing the relationship between attachment, parental attitude and plasma oxytocin in schizophrenia patients and their unaffected siblings. Nordic journal of psychiatry, 73, 51-57. https://doi.org/10.1080/08039488.2018.1554698

Bailey, T., Alvarez-Jimenez, M., Garcia-Sanchez, A. M., Hulbert, C., Barlow, E. y Bendall, S. (2018). Childhood Trauma Is Associated With Severity of Hallucinations and Delusions in Psychotic Disorders: A Systematic Review and Meta-Analysis. Schizophrenia bulletin, 44(5), 1111-1122.

Balluerka, N., Lacasa, F., Gorostiaga, A., Muela, A. y Pierrehumbert, B. (2011). Versión reducida del cuestionario Camir (Camir-R) para la evaluación del apego. Psicothema, 23.

Barbato, M., Colijn, M. A., Keefe, R. S., Perkins, D. O., Woods, S. W., Hawkins, K. A., Christensen, B. K. y Addington, J. (2013). The course of cognitive functioning over six months in individuals at clinical high risk for psychosis. Psychiatry research, 206(2-3), 195-199. https://doi.org/10.1016/j.psychres.2012.10.013

Barch, D. M., Bustillo, J., Gaebel, W., Gur, R., Heckers, S., Malaspina, D., Owen, M. J., Schultz, S., Tandon, R., Tsuang, M., Van Os, J. y Carpenter, W. (2013). Logic and justification for dimensional assessment of symptoms and related clinical phenomena in psychosis: 
relevance to DSM-5. Schizophrenia research, 150(1), 15-20. https://doi.org/10.1016/ j.schres.2013.04.027

Barch, D. M. y Ceaser, A. (2012). Cognition in schizophrenia: core psychological and neural mechanisms. Trends in cognitive sciences, 16(1), 27-34. https://doi.org/ 10.1016/j.tics.2011.11.015

Bartholomew, K. y Horowitz, L. M. (1991). Attachment styles among young adults: A test of a four-category model. Journal of Personality and Social Psychology, 61(2), 226244. https://doi.org/10.1037/0022-3514.61.2.226

Bäuml, J., Froböse, T., Kraemer, S., Rentrop, M. y Pitschel-Walz, G. (2006). Psychoeducation: a basic psychotherapeutic intervention for patients with schizophrenia and their families. Schizophrenia bulletin, 32 Suppl 1(Suppl 1), S1-S9. https://doi.org/10.1093/schbul/sbl017

Beck, A., Himelstein, R., Bredemeier, K., Silverstein, S. y Grant, P. (2018). What accounts for poor functioning in people with schizophrenia: a re-evaluation of the contributions of neurocognitive v. attitudinal and motivational factors. Psychological medicine, 48 16, 2776-2785 .

Beck, A. T. y Rector, N. A. (2005). Cognitive approaches to schizophrenia: theory and therapy. Annual review of clinical psychology, 1, 577-606. https://doi.org/10.1146/ annurev.clinpsy.1.102803.144205

Beck, A. T., Rector, N. A., Stolar, N. y Grant, P. (2009). Schizophrenia: Cognitive Theory, Research, and Therapy. (p. 418). New York, NY: Guilford Publications.

Beck, A. T., Ward, C. H., Mendelson, M., Mock, J. y Erbaugh, J. (1961). An inventory for measuring depression. Archives of general psychiatry, 4, 561-571. https://doi.org/ 10.1001/archpsyc.1961.01710120031004

Beck Institute (2019). CBT for schizophrenia. Bala Cynwyd, PA: Beck Institute.

Bentall, R. P., Corcoran, R., Howard, R., Blackwood, N. y Kinderman, P. (2001). Persecutory delusions: a review and theoretical integration. Clinical psychology review, 21(8), 1143-1192. https://doi.org/10.1016/s0272-7358(01)00106-4

Berry, K., Barrowclough, C. y Wearden, A. (2008). Attachment theory: a framework for understanding symptoms and interpersonal relationships in psychosis. Behaviour research and therapy, 46(12), 1275-1282. https://doi.org/10.1016/j.brat. 2008.08.009 
Berry, K., Wearden, A., Barrowclough, C., Oakland, L. y Bradley, J. (2012). An investigation of adult attachment and the nature of relationships with voices. The British journal of clinical psychology, 51(3), 280-291. https://doi.org/10.1111/j. 2044-8260.2011.02027.x

Biedermann, F. y Fleischhacker, W. W. (2016). Psychotic disorders in DSM-5 and ICD-11. CNS spectrums, 21(4), 349-354. https://doi.org/10.1017/S1092852916000316

Biesanz, J. C., Falk, C. F. y Savalei, V. (2010). Assessing Mediational Models: Testing and Interval Estimation for Indirect Effects. Multivariate Behavioral Research, 45, 661701. https://doi.org/10.1080/00273171.2010.498292

Bifulco, A. y Thomas, G. (2012). Understanding Adult Attachment in Family Relationships: Research, Assessment and Intervention (1st Edition). London: Routledge. Taylor \& Francis Group. http://doi.org/https://doi.org/10.4324/9780203094556

Billeke, P. y Aboitiz, F. (2013). Social cognition in schizophrenia: from social stimuli processing to social engagement. Frontiers in psychiatry, 4, 4. https://doi.org/ 10.3389/fpsyt.2013.00004

Blair, M. A., Nitzburg, G., DeRosse, P. y Karlsgodt, K. H. (2018). Relationship between executive function, attachment style, and psychotic-like experiences in typically developing youth. Schizophrenia Research, 197, 428-433. https://doi.org/10.1016/ j.schres.2018.02.026

Bonoldi, I., Simeone, E., Rocchetti, M., Codjoe, L., Rossi, G., Gambi, F., Balottin, U., Caverzasi, E., Politi, P. y Fusar-Poli, P. (2013). Prevalence of self-reported childhood abuse in psychosis: a meta-analysis of retrospective studies. Psychiatry research, 210(1), 8-15. https://doi.org/10.1016/j.psychres.2013.05.003

Bora, E., Binnur Akdede, B. y Alptekin, K. (2017). Neurocognitive impairment in deficit and non-deficit schizophrenia: a meta-analysis. Psychological medicine, 47(14), 24012413. https://doi.org/10.1017/S0033291717000952

Borsboom, D. y Cramer, A. O. (2013). Network analysis: an integrative approach to the structure of psychopathology. Annual review of clinical psychology, 9, 91-121. https:// doi.org/10.1146/annurev-clinpsy-050212-185608

Bowlby, J. (1969). Attachment and Loss (3 Vols). New York, NY: Basic Books. 
Bowlby, J. (1977). The making and breaking of affectional bonds: I. Aetiology and psychopathology in the light of attachment theory. The British Journal of Psychiatry, 130, 201-210. https://doi.org/10.1192/bjp.130.3.201

Bowlby, J. (1980). Attachment and Loss (Vol 3): Loss, Sadness and Depression. New York, NY: Basic Books.

Bowlby, J. (1982). Attachment and Loss (Vol 1): Attachment. New York, NY: Basic Books.

Bowlby, J. (1988). A Secure Base: Clinical Applications of Attachment Theory. London: Routledge.

Brennan, K. A., Clark, C. L. y Shaver, P. R. (1998). Self-report measurement of adult attachment: An integrative overview. En J. A. Simpson \& W. S. Rholes (Eds.), Attachment theory and close relationships (p. 46-76). The Guilford Press.

Brenner, H. D., Hodel, B., Roder, V. y Corrigan, P. (1992). Treatment of cognitive dysfunctions and behavioral deficits in schizophrenia. Schizophrenia bulletin, 18(1), 21-26. https://doi.org/10.1093/schbul/18.1.21

Brekke, J., Kay, D. D., Lee, K. S. y Green, M. F. (2005). Biosocial pathways to functional outcome in schizophrenia. Schizophrenia research, 80(2-3), 213-225. https://doi.org/ 10.1016/j.schres.2005.07.008

Bringmann, L. F., Elmer, T., Epskamp, S., Krause, R. W., Schoch, D., Wichers, M., Wigman, J. T. W. y Snippe, E. (2019). What do centrality measures measure in psychological networks? Journal of Abnormal Psychology, 128(8), 892-903. https://doi.org/ $10.1037 / \mathrm{abn} 0000446$

Brown, E. C., Tas, C. y Brüne, M. (2012). Potential therapeutic avenues to tackle social cognition problems in schizophrenia. Expert review of neurotherapeutics, 12(1), 7181. https://doi.org/10.1586/ern.11.183

Brown, E. C., Tas, C., Can, H., Esen-Danaci, A. y Brüne, M. (2014). A closer look at the relationship between the subdomains of social functioning, social cognition and symptomatology in clinically stable patients with schizophrenia. Comprehensive psychiatry, 55(1), 25-32. https://doi.org/10.1016/j.comppsych.2013.10.001

Browne, J., Penn, D. L., Raykov, T., Pinkham, A. E., Kelsven, S., Buck, B. y Harvey, P. D. (2016). Social cognition in schizophrenia: Factor structure of emotion processing and theory of mind. Psychiatry research, 242, 150-156. https://doi.org/10.1016/ j.psychres.2016.05.034 
Bucci P. (2017). ICD-11 draft diagnostic guidelines open to input by mental health professionals. World psychiatry : official journal of the World Psychiatric Association (WPA), 16(1), 115-116. https://doi.org/10.1002/wps.20402

Buck, B. E., Healey, K. M., Gagen, E. C., Roberts, D. L. y Penn, D. L. (2016). Social cognition in schizophrenia: factor structure, clinical and functional correlates. Journal of mental health (Abingdon, England), 25(4), 330-337. https://doi.org/ 10.3109/09638237.2015.1124397

Brüne M. (2005). "Theory of mind" in schizophrenia: a review of the literature. Schizophrenia bulletin, 31(1), 21-42. https://doi.org/10.1093/schbul/sbi002

Brüne, M., Ozgürdal, S., Ansorge, N., von Reventlow, H. G., Peters, S., Nicolas, V., Tegenthoff, M., Juckel, G. y Lissek, S. (2011). An fMRI study of "theory of mind" in atrisk states of psychosis: comparison with manifest schizophrenia and healthy controls. NeuroImage, 55(1), 329-337. https://doi.org/10.1016/j.neuroimage. 2010.12.018

Brüne, M., Schaub, D., Juckel, G. y Langdon, R. (2011). Social skills and behavioral problems in schizophrenia: the role of mental state attribution, neurocognition and clinical symptomatology. Psychiatry research, 190(1), 9-17. https://doi.org/10.1016/ j.psychres.2010.03.015

Bryson, G., Bell, M. y Lysaker, P. (1997). Affect recognition in schizophrenia: a function of global impairment or a specific cognitive deficit. Psychiatry research, 71(2), 105-113. https://doi.org/10.1016/s0165-1781(97)00050-4

Buchy, L., Woodward, T. S. y Liotti, M. (2007). A cognitive bias against disconfirmatory evidence (BADE) is associated with schizotypy. Schizophrenia research, 90(1-3), 334337. https://doi.org/10.1016/j.schres.2006.11.012

Catalan, A., Angosto, V., Díaz, A., Martínez, N., Guede, D., Pereda, M., Madrazo, A., Bustamante, S., Bilbao, A., Osa, L., Inchausti, L. y Gonzalez-Torres, M. A. (2018). The relationship between theory of mind deficits and neurocognition in first episodepsychosis. Psychiatry research, 268, 361-367. https://doi.org/10.1016/j.psychres. 2018.06.066

Catley, D., Goggin, K., Harris, K. J., Richter, K. P., Williams, K., Patten, C., Resnicow, K., Ellerbeck, E. F., Bradley-Ewing, A., Lee, H. S., Moreno, J. L. y Grobe, J. E. (2016). A Randomized Trial of Motivational Interviewing: Cessation Induction Among Smokers 
With Low Desire to Quit. American journal of preventive medicine, 50(5), 573-583. https://doi.org/10.1016/j.amepre.2015.10.013

Chatziioannidis, S., Andreou, C., Agorastos, A., Kaprinis, S., Malliaris, Y., Garyfallos, G. y Bozikas, V. P. (2019). The role of attachment anxiety in the relationship between childhood trauma and schizophrenia-spectrum psychosis. Psychiatry research, 276, 223-231. https://doi.org/10.1016/j.psychres.2019.05.021

Carr, S. C., Hardy, A. y Fornells-Ambrojo, M. (2018). Relationship between attachment style and symptom severity across the psychosis spectrum: A meta-analysis. Clinical psychology review, 59, 145-158. https://doi.org/10.1016/j.cpr.2017.12.001

Cohen, A. N., Drapalski, A. L., Glynn, S. M., Medoff, D., Fang, L. J. y Dixon, L. B. (2013). Preferences for family involvement in care among consumers with serious mental illness. Psychiatric services (Washington, D.C.), 64(3), 257-263. https://doi.org/ 10.1176/appi.ps.201200176

Combs, D. R., Elerson, K., Penn, D. L., Tiegreen, J. A., Nelson, A., Ledet, S. N. y Basso, M. R. (2009). Stability and generalization of Social Cognition and Interaction Training (SCIT) for schizophrenia: six-month follow-up results. Schizophrenia research, 112(1-3), 196-197. https://doi.org/10.1016/j.schres.2009.04.010

Combs, D. R., Penn, D. L., Wicher, M. y Waldheter, E. (2007). The Ambiguous Intentions Hostility Questionnaire (AIHQ): a new measure for evaluating hostile social-cognitive biases in paranoia. Cognitive neuropsychiatry, 12(2), 128-143. https://doi.org/ $10.1080 / 13546800600787854$

Contreras, A., Nieto, I., Valiente, C., Espinosa, R. y Vazquez, C. (2019). The study of psychopathology from the network analysis perspective: A systematic review. Psychotherapy and Psychosomatics, 88, 71-83. https://doi.org/10.1159/000497425

Corcoran, R., Mercer, G. y Frith, C. D. (1995). Schizophrenia, symptomatology and social inference: investigating "theory of mind" in people with schizophrenia. Schizophrenia research, 17(1), 5-13. https://doi.org/10.1016/0920-9964(95)00024-g

Correll, C. U., Citrome, L., Haddad, P. M., Lauriello, J., Olfson, M., Calloway, S. M. y Kane, J. M. (2016). The Use of Long-Acting Injectable Antipsychotics in Schizophrenia: Evaluating the Evidence. The Journal of clinical psychiatry, 77(suppl 3), 1-24. https:// doi.org/10.4088/JCP.15032su1 
Cotter, J., Bartholomeusz, C., Papas, A., Allott, K., Nelson, B., Yung, A. R. y Thompson, A. (2017). Examining the association between social cognition and functioning in individuals at ultra-high risk for psychosis. Australian \& New Zealand Journal of Psychiatry, 51(1), 83-92. https://doi.org/10.1177/0004867415622691

Crowell, J. A. (2014). The adult attachment interview. En S. Farnfield, P. Holmes (Eds). The Routledge Handbook of Attachment: Assessment (p. 144-55). London: Routledge.

Crowell, J. A. y Treboux, D. (1995). A review of adult attachment measures: Implications for theory and research. Social Development, 4(3), 294-327. https://doi.org/ 10.1111/j.1467-9507.1995.tb00067.x

Davidson, C. A., Lesser, R., Parente, L. T. y Fiszdon, J. M. (2018). Psychometrics of social cognitive measures for psychosis treatment research. Schizophrenia Research, 193, 51-57. https://doi.org/10.1016/J.SCHRES.2017.06.018

Debbané, M., Salaminios, G., Luyten, P., Badoud, D., Armando, M., Solida Tozzi, A., Fonagy, P. y Brent, B. K. (2016). Attachment, Neurobiology, and Mentalizing along the Psychosis Continuum. Frontiers in human neuroscience, 10, 406. https://doi.org/ 10.3389/fnhum.2016.00406

de Jong, S., van Donkersgoed, R., Timmerman, M. E., Aan Het Rot, M., Wunderink, L., Arends, J., van Der Gaag, M., Aleman, A., Lysaker, P. H. y Pijnenborg, G. (2019). Metacognitive reflection and insight therapy (MERIT) for patients with schizophrenia. Psychological medicine, 49(2), 303-313. https://doi.org/10.1017/ S0033291718000855

de Vries, B., van Busschbach, J. T., van der Stouwe, E., Aleman, A., van Dijk, J., Lysaker, P. H., Arends, J., Nijman, S. A. y Pijnenborg, G. (2019). Prevalence Rate and Risk Factors of Victimization in Adult Patients With a Psychotic Disorder: A Systematic Review and Meta-analysis. Schizophrenia bulletin, 45(1), 114-126. https://doi.org/10.1093/ schbul/sby020

Donohoe, G., Spoletini, I., McGlade, N., Behan, C., Hayden, J., O'Donoghue, T., Peel, R., Haq, F., Walker, C., O'Callaghan, E., Spalletta, G., Gill, M. y Corvin, A. (2008). Are relational style and neuropsychological performance predictors of social attributions in chronic schizophrenia?. Psychiatry research, 161(1), 19-27. https://doi.org/10.1016/ j.psychres.2007.10.001 
Drapalski, A. L., Piscitelli, S., Lee, R. J., Medoff, D. y Dixon, L. B. (2018). Family Involvement in the Clinical Care of Clients With First-Episode Psychosis in the RAISE Connection Program. Psychiatric services (Washington, D.C.), 69(3), 358-361. https:// doi.org/10.1176/appi.ps.201700080

Dudley, R. E. J. y Over, D. E. (2003). People with delusions jump to conclusions: a theoretical account of research findings on the reasoning of people with delusions. Clinical Psychohology \& Psychotherapy, 10(5), 263-274. https://doi.org/10.1002/cpp. 376

Easter, M. M., Swanson, J. W., Robertson, A. G., Moser, L. L. y Swartz, M. S. (2017). Facilitation of Psychiatric Advance Directives by Peers and Clinicians on Assertive Community Treatment Teams. Psychiatric services (Washington, D.C.), 68(7), 717-723. https://doi.org/10.1176/appi.ps.201600423

Eichner, C. y Berna, F. (2016). Acceptance and Efficacy of Metacognitive Training (MCT) on Positive Symptoms and Delusions in Patients With Schizophrenia: A Meta-analysis Taking Into Account Important Moderators. Schizophrenia bulletin, 42(4), 952-962. https://doi.org/10.1093/schbul/sbv225

Epskamp, S., Borsboom, D. y Fried, E. I. (2018). Estimating psychological networks and their accuracy: A tutorial paper. Behavior Research Methods, 50(1), 195-212. https:// doi.org/10.3758/s13428-017-0862-1

Extremera, N. y Fernández-Berrocal, P. (2009). Test de Inteligencia Emocional de MayerSalovey-Caruso [Spanish Mayer-Salovey-Caruso Emotional Intelligence Test]. TEA Ediciones: Madrid.

Fanning, J. R., Bell, M. D. y Fiszdon, J. M. (2012). Is it possible to have impaired neurocognition but good social cognition in schizophrenia?. Schizophrenia research, 135(1-3), 68-71. https://doi.org/10.1016/j.schres.2011.12.009

Favrod, J., Rexhaj, S., Bardy, S., Ferrari, P., Hayoz, C., Moritz, S., Conus, P. y Bonsack, C. (2014). Sustained antipsychotic effect of metacognitive training in psychosis: a randomized-controlled study. European psychiatry : the journal of the Association of European Psychiatrists, 29(5), 275-281. https://doi.org/10.1016/j.eurpsy. 2013.08.003

Fernández Fuertes, A. A., Orgaz, B., Fuertes, A. y Carcedo, R. (2011). La evaluación del apego romántico en adolescentes españoles: validación de la versión reducida del 
Experiences in Close Relationships-Revised (ECR-R). Anales De Psicología / Annals of Psychology, 27(3), 827-833. Recuperado a partir de https://revistas.um.es/analesps/ article/view/135561

Ferrando, S. J., Owen, J. A. y Levenson, J. L. (2014). Psychopharmacology. En R. E. Hales, S. C. Yudofsky y L. W. Roberts: The American Psychiatric Publishing Textbook of Psychiatry (6th Ed.) (pp. 929-1003). Washington, DC: American Psychiatric Publishing.

Fett, A. K., Viechtbauer, W., Dominguez, M. D., Penn, D. L., van Os, J. y Krabbendam, L. (2011). The relationship between neurocognition and social cognition with functional outcomes in schizophrenia: a meta-analysis. Neuroscience and biobehavioral reviews, 35(3), 573-588. https://doi.org/10.1016/j.neubiorev. 2010.07.001

Fine, C., Gardner, M., Craigie, J. y Gold, I. (2007). Hopping, skipping or jumping to conclusions? Clarifying the role of the JTC bias in delusions. Cognitive neuropsychiatry, 12(1), 46-77. https://doi.org/10.1080/13546800600750597

Flavell, J. H. (1979). Metacognition and cognitive monitoring: A new area of cognitivedevelopmental inquiry. American Psychologist, 34(10), 906-911. https://doi.org/ 10.1037/0003-066X.34.10.906

Fontanil Gómez, Y., Ezama Coto, E. y Alonso, Y. (2013). Validation of the Scale of Preferences and Expectations in Close Interpersonal Relationships (EPERIC). Psicothema, 25, 275-281. http://doi.org/10.7334/psicothema2012.125

Fraley, R. C., Waller, N. G. y Brennan, K. A. (2000). An item response theory analysis of self-report measures of adult attachment. Journal of personality and social psychology, 78(2), 350-365. https://doi.org/10.1037//0022-3514.78.2.350

Freeman D. (2007). Suspicious minds: the psychology of persecutory delusions. Clinical psychology review, 27(4), 425-457. https://doi.org/10.1016/j.cpr.2006.10.004

Fretland, R. A., Andersson, S., Sundet, K., Andreassen, O. A., Melle, I. y Vaskinn, A. (2015). Theory of mind in schizophrenia: error types and associations with symptoms. Schizophrenia research, 162(1-3), 42-46. https://doi.org/10.1016/j.schres. 2015.01.024

Fried, E. I., van Borkulo, C. D., Cramer, A. O. J., Boschloo, L., Schoevers, R. A. y Borsboom, D. (2017). Mental disorders as networks of problems: a review of recent insights. 
Social Psychiatry and Psychiatric Epidemiology, 52, 1-10. https://doi.org/10.1007/ s00127-016-1319-z

Friedman, J., Hastie, T. y Tibshirani, R. (2010). Applications of the lasso and grouped lasso to the estimation of sparse graphical models. Technical Report, 1-22.

Fruchterman, T. M. J. y Reingold, E. M. (1991). Graph drawing by force-directed placement. Software: Practice and Experience, 21(11), 1129-1164. https://doi.org/ $10.1002 /$ spe.4380211102

Gaebel, W. (2012). Status of Psychotic Disorders in ICD-11. Schizophrenia Bulletin, 38(5), 895-898. https://doi.org/10.1093/schbul/sbs133

Gaebel, W., Zielasek, J. y Cleveland, H. R. (2013). Psychotic disorders in ICD-11. Asian journal of psychiatry, 6(3), 263-265. https://doi.org/10.1016/j.ajp.2013.04.002

García, R. R., Aliste, F. y Soto, G. (2018). Social Cognition in Schizophrenia: Cognitive and Neurobiological Aspects. Cognición social en esquizofrenia: aspectos cognitivos y neurobiológicos. Revista colombiana de psiquiatria, 47(3), 170-176. https://doi.org/ 10.1016/j.rcp.2017.03.004

Garety, P. A., Bebbington, P., Fowler, D., Freeman, D. y Kuipers, E. (2007). Implications for neurobiological research of cognitive models of psychosis: a theoretical paper. Psychological medicine, 37(10), 1377-1391. https://doi.org/10.1017/ S003329170700013X

Garety, P. A. y Freeman, D. (2013). The past and future of delusions research: from the inexplicable to the treatable. The British journal of psychiatry: the journal of mental science, 203(5), 327-333. https://doi.org/10.1192/bjp.bp.113.126953

Garety, P. A., Freeman, D., Jolley, S., Dunn, G., Bebbington, P. E., Fowler, D. G. y Dudley, R. (2005). Reasoning, emotions, and delusional conviction in psychosis. Journal of Abnormal Psychology, 114, 373-384. doi:10.1037/0021-843X.114.3.373

Gawęda, Ł., Pionke, R., Krężołek, M., Prochwicz, K., Kłosowska, J., Frydecka, D., Misiak, B., Kotowicz, K., Samochowiec, A., Mak, M., Błądziński, P., Cechnicki, A. y Nelson, B. (2018). Self-disturbances, cognitive biases and insecure attachment as mechanisms of the relationship between traumatic life events and psychotic-like experiences in non-clinical adults - A path analysis. Psychiatry research, 259, 571-578. https:// doi.org/10.1016/j.psychres.2017.11.009 
George, C., Kaplan, N. \& Main, M. (1985). The Adult Attachment Interview. Unpublished manuscript, University of California, Berkeley.

Gil, D., Fernández-Modamio, M., Bengochea, R. y Arrieta, M. (2012). Adaptación al español de la prueba de teoría de la mente Hinting Task. Revista de Psiquiatría y Salud Mental, 5, 79-88. https://doi.org/10.1016/j.rpsm.2011.11.004

Gil-Sanz, D., Fernández-Modamio, M., Bengochea-Seco, R., Arrieta-Rodríguez, M. y PérezFuentes, G. (2016). Efficacy of the Social Cognition Training Program in a Sample of Outpatients with Schizophrenia. Clinical schizophrenia \& related psychoses, 10(3), 154-162. https://doi.org/10.3371/1935-1232.10.3.154

Gil-Sanz, D., Fernández-Modamio, M., Bengochea-Seco, R., Arrieta-Rodríguez, M., González-Fraile, E., Pérez-Fuentes, G., García-Polavieja, B., Martín-Carrasco, M., de Tojeiro-Roce, J. G. y Santos-Zorrozúa, B. (2017). PERE: Una nueva herramienta para valorar el reconocimiento de las emociones básicas y su aplicación en la esquizofrenia [ERAT: A new tool to assess basic emotions recognition and its application in schizophrenia]. Revista de Psicopatología y Psicología Clínica, 22(2), 85-93. https://doi.org/10.5944/rppc.vol.22.num.2.2017.17244

Gómez-Gastiasoro, A., Zubiaurre-Elorza, L., Peña, J., Ibarretxe-Bilbao, N., Rilo, O., Schretlen, D. J. y Ojeda, N. (2019). Altered frontal white matter asymmetry and its implications for cognition in schizophrenia: A tractography study. NeuroImage. Clinical, 22, 101781. https://doi.org/10.1016/j.nicl.2019.101781

González, L. E., López-Carrilero, R., Barrigón, M. L., Grasa, E., Barajas, A., Pousa, E., González-Higueras, F., Ruiz-Delgado, I., Cid, J., Lorente-Rovira, E., Pélaez, T., Spanish Metacognition Study Group y Ochoa, S. (2018). Neuropsychological functioning and jumping to conclusions in recent onset psychosis patients. Schizophrenia research, 195, 366-371. https://doi.org/10.1016/j.schres.2017.09.039

Green, M. F., Bearden, C. E., Cannon, T. D., Fiske, A. P., Hellemann, G. S., Horan, W. P., Kee, K., Kern, R. S., Lee, J., Sergi, M. J., Subotnik, K. L., Sugar, C. A., Ventura, J., Yee, C. M. y Nuechterlein, K. H. (2012). Social cognition in schizophrenia, Part 1: performance across phase of illness. Schizophrenia bulletin, 38(4), 854-864. https://doi.org/ $10.1093 /$ schbul/sbq171

Green, M. F., Horan, W. P. y Lee, J. (2015). Social cognition in schizophrenia. Nature reviews. Neuroscience, 16(10), 620-631. https://doi.org/10.1038/nrn4005 
Green, M. F., Horan, W. P., Lee, J., McCleery, A., Reddy, L. F. y Wynn, J. K. (2018). Social Disconnection in Schizophrenia and the General Community. Schizophrenia bulletin, 44(2), 242-249. https://doi.org/10.1093/schbul/sbx082

Green, M. F. y Nuechterlein, K. H. (1999). Should schizophrenia be treated as a neurocognitive disorder?. Schizophrenia bulletin, 25(2), 309-319. https://doi.org/ 10.1093/oxfordjournals.schbul.a033380

Green, M. F., Olivier, B., Crawley, J. N., Penn, D. L. y Silverstein, S. (2005). Social cognition in schizophrenia: recommendations from the measurement and treatment research to improve cognition in schizophrenia new approaches conference. Schizophrenia bulletin, 31(4), 882-887. https://doi.org/10.1093/schbul/sbi049

Green, M. F., Penn, D. L., Bentall, R., Carpenter, W. T., Gaebel, W., Gur, R. C., Kring, A. M., Park, S., Silverstein, S. M. y Heinssen, R. (2008). Social cognition in schizophrenia: an NIMH workshop on definitions, assessment, and research opportunities. Schizophrenia bulletin, 34(6), 1211-1220. https://doi.org/10.1093/schbul/sbm145

Griffin, D. W. y Bartholomew, K. (1994). The metaphysics of measurement: The case of adult attachment. En K. Bartholomew \& D. Perlman (Eds.), Advances in personal relationships, Vol. 5. Attachment processes in adulthood (p. 17-52). Jessica Kingsley Publishers.

Gumley, A. I., Taylor, H. E., Schwannauer, M. y MacBeth, A. (2014). A systematic review of attachment and psychosis: measurement, construct validity and outcomes. Acta psychiatrica Scandinavica, 129(4), 257-274. https://doi.org/10.1111/acps.12172

Haeffel, G. J., Hershenberg, R., Goodson, J. T., Hein, S., Square, A., Grigorenko, E. L. y Chapman, J. (2017). The hopelessness theory of depression: Clinical utility and generalizability. Cognitive Therapy and Research, 41, 543-555. https://doi.org/ 10.1007/s10608-017-9833-1

Hajdúk, M., Harvey, P. D., Penn, D. L. y Pinkham, A. E. (2018). Social cognitive impairments in individuals with schizophrenia vary in severity. Journal of psychiatric research, 104, 65-71. https://doi.org/10.1016/j.jpsychires.2018.06.017

Hamann, J. y Heres, S. (2019). Why and How Family Caregivers Should Participate in Shared Decision Making in Mental Health. Psychiatric services (Washington, D.C.), 70(5), 418-421. https://doi.org/10.1176/appi.ps.201800362 
Harder, S. (2014). Attachment in schizophrenia - implications for research, prevention, and treatment. Schizophrenia bulletin, 40(6), 1189-1193. https://doi.org/10.1093/ schbul/sbu133

Hardey, K. (2019). Cognitive behavioral therapy for psychosis (CBTp). Alexandria, VA: National Association of State Mental Health Program Directors.

Harrow, M., Jobe, T.H., Grossman, L. y Faull, R. (2015). The continuous administration of antipsychotic medications over 20-years for schizophrenia: Relapse and recovery. Schizophrenia Bulletin, 41(1), 341. https://dx.doi.org/10.1093/schbul/sbv010

Harvey, P. D. (2011). Assessment of everyday functioning in schizophrenia. Innovations in clinical neuroscience, 8(5), 21-24.

Harvey, P. D., McGurk, S. R., Mahncke, H. y Wykes, T. (2018). Controversies in Computerized Cognitive Training. Biological psychiatry. Cognitive neuroscience and neuroimaging, 3(11), 907-915. https://doi.org/10.1016/j.bpsc.2018.06.008

Harvey, P. D. y Penn, D. (2010). Social cognition: the key factor predicting social outcome in people with schizophrenia?. Psychiatry (Edgmont (Pa. : Township)), 7(2), 41-44.

Hasson-Ohayon, I., Avidan-Msika, M., Mashiach-Eizenberg, M., Kravetz, S., Rozencwaig, S., Shalev, H. y Lysaker, P. H. (2015). Metacognitive and social cognition approaches to understanding the impact of schizophrenia on social quality of life. Schizophrenia research, 161(2-3), 386-391. https://doi.org/10.1016/j.schres.2014.11.008

Hasson-Ohayon, I., Goldzweig, G., Lavi-Rotenberg, A., Luther, L. y Lysaker, P. H. (2018). The centrality of cognitive symptoms and metacognition within the interacting network of symptoms, neurocognition, social cognition and metacognition in schizophrenia. Schizophrenia research, 202, 260-266. https://doi.org/10.1016/ j.schres.2018.07.007

Hazan, C. y Shaver, P. (1987). Romantic love conceptualized as an attachment process. Journal of Personality and Social Psychology, 52(3), 511-524. https://doi.org/ 10.1037/0022-3514.52.3.511

Helfer, B., Samara, M. T., Huhn, M., Klupp, E., Leucht, C., Zhu, Y., Engel, R. R. y Leucht, S. (2016). Efficacy and Safety of Antidepressants Added to Antipsychotics for Schizophrenia: A Systematic Review and Meta-Analysis. The American journal of psychiatry, 173(9), 876-886. https://doi.org/10.1176/appi.ajp.2016.15081035 
Hesse, E. (2008). The adult attachment interview: protocol, method of analysis, and empirical studies. En J. E. Cassidy y P. R. Shaver (Eds.) Handbook of Attachment: Theory, Research, and Clinical Applications (pp. 552-599). New York, NY: Guildford Press.

Hill, S. K., Reilly, J. L., Harris, M. S., Rosen, C., Marvin, R. W., Deleon, O. y Sweeney, J. A. (2009). A comparison of neuropsychological dysfunction in first-episode psychosis patients with unipolar depression, bipolar disorder, and schizophrenia. Schizophrenia research, 113(2-3), 167-175. https://doi.org/10.1016/j.schres.2009.04.020

Hogarty, G. E. y Flesher, S. (1999). Developmental theory for a cognitive enhancement therapy of schizophrenia. Schizophrenia bulletin, 25(4), 677-692. https://doi.org/ 10.1093/oxfordjournals.schbul.a033410

Horan, W. P., Blanchard, J. J., Clark, L. A. y Green, M. F. (2008). Affective traits in schizophrenia and schizotypy. Schizophrenia bulletin, 34(5), 856-874. https:// doi.org/10.1093/schbul/sbn083

Horan, W. P. y Green, M. F. (2019). Treatment of social cognition in schizophrenia: Current status and future directions. Schizophrenia research, 203, 3-11. https:// doi.org/10.1016/j.schres.2017.07.013

Horan, W. P., Green, M. F., DeGroot, M., Fiske, A., Hellemann, G., Kee, K., Kern, R. S., Lee, J., Sergi, M. J., Subotnik, K. L., Sugar, C. A., Ventura, J. y Nuechterlein, K. H. (2012). Social cognition in schizophrenia, Part 2: 12-month stability and prediction of functional outcome in first-episode patients. Schizophrenia bulletin, 38(4), 865-872. https:// doi.org/10.1093/schbul/sbr001

Howes, O. D., McCutcheon, R., Agid, O., de Bartolomeis, A., van Beveren, N. J., Birnbaum, M. L., Bloomfield, M. A., Bressan, R. A., Buchanan, R. W., Carpenter, W. T., Castle, D. J., Citrome, L., Daskalakis, Z. J., Davidson, M., Drake, R. J., Dursun, S., Ebdrup, B. H., Elkis, H., Falkai, P., Fleischacker, W. W., ... Correll, C. U. (2017). Treatment-Resistant Schizophrenia: Treatment Response and Resistance in Psychosis (TRRIP) Working Group Consensus Guidelines on Diagnosis and Terminology. The American journal of psychiatry, 174(3), 216-229. https://doi.org/10.1176/appi.ajp.2016.16050503

Howes, O. D. y Murray, R. M. (2014). Schizophrenia: an integrated sociodevelopmentalcognitive model. Lancet (London, England), 383(9929), 1677-1687. https://doi.org/ 10.1016/S0140-6736(13)62036-X 
Hu, L. T. y Bentler, P. M. (1999). Cutoff criteria for Fit indexes in covariance structure analysis: Conventional criteria versus new alternatives. Structural Equation Modeling, 6, 1-55. https://doi.org/10.1080/10705519909540118

Hünefeldt, T., Laghi, F., Ortu, F. y Belardinelli, M. O. (2013). The relationship between 'theory of mind' and attachment-related anxiety and avoidance in Italian adolescents. Journal of adolescence, 36(3), 613-621. https://doi.org/10.1016/j.adolescence. 2013.03.012

Ihara, K., Morgan, C., Fearon, P., Dazzan, P., Demjaha, A., Lloyd, T., Kirkbride, J. B., Hayhurst, H., Murray, R. M. y Jones, P. B. (2009). The prevalence, diagnostic significance and demographic characteristics of Schneiderian first-rank symptoms in an epidemiological sample of first-episode psychoses. Psychopathology, 42(2), 81-91. https://doi.org/10.1159/000203340

Isaac, C. y Januel, D. (2016). Neural correlates of cognitive improvements following cognitive remediation in schizophrenia: a systematic review of randomized trials. Socioaffective neuroscience \& psychology, 6, 30054. https://doi.org/10.3402/ snp.v6.30054

Isvoranu, A. M., Van Borkulo, C. D., Boyette, L. Lou, Wigman, J. T. W., Vinkers, C. H., Borsboom, D., ... Myin-Germeys, I. (2017). A network approach to psychosis: Pathways between childhood trauma and psychotic symptoms. Schizophrenia Bulletin, 43, 187-196. https://doi.org/10.1093/schbul/sbw055

Jabben, N., Arts, B., van Os, J. y Krabbendam, L. (2010). Neurocognitive Functioning as Intermediary Phenotype and Predictor of Psychosocial Functioning Across the Psychosis Continuum: Studies in Schizophrenia and Bipolar Disorder. The Journal of Clinical Psychiatry, 71, 764-774. http://doi.org/10.4088/JCP.08m04837yel

Jackson G. E. (2008). Chemo brain - a psychotropic drug phenomenon?. Medical hypotheses, 70(3), 572-577. https://doi.org/10.1016/j.mehy.2007.06.019

James, A. V., Hasson-Ohayon, I., Vohs, J., Minor, K. S., Leonhardt, B. L., Buck, K. D., George, S. y Lysaker, P. H. (2016). Metacognition moderates the relationship between dysfunctional self-appraisal and social functioning in prolonged schizophrenia independent of psychopathology. Comprehensive psychiatry, 69, 62-70. https:// doi.org/10.1016/j.comppsych.2016.05.008

JASP Team (2020). JASP (Version 0.13.1) [Computer software]. https://jasp-stats.org/ 
Javed, A. y Charles, A. (2018). The Importance of Social Cognition in Improving Functional Outcomes in Schizophrenia. Frontiers in psychiatry, 9, 157. https:// doi.org/10.3389/fpsyt.2018.00157

Jirsaraie, R. J., Sheffield, J. M. y Barch, D. M. (2018). Neural correlates of global and specific cognitive deficits in schizophrenia. Schizophrenia research, 201, 237-242. https://doi.org/10.1016/j.schres.2018.06.017

Jobe, T. H. y Harrow, M. (2010). Schizophrenia course, long-term outcome, recovery, and prognosis. Current Directions in Psychological Science, 19(4), 220-225. https:// doi.org/10.1177/0963721410378034

Jones, P. J., Mair, P., Riemann, B. C., Mugno, B. L., y McNally, R. J. (2018). A network perspective on comorbid depression in adolescents with obsessive-compulsive disorder. Journal of Anxiety Disorders, 53, 1-8. https://doi.org/https://doi.org/ 10.1016/j.janxdis.2017.09.008

Juárez-Ramos, V., Rubio, J. L., Delpero, C., Mioni, G., Stablum, F. y Gómez-Milán, E. (2014). Jumping to Conclusions bias, BADE and Feedback Sensitivity in schizophrenia and schizotypy. Consciousness and cognition, 26, 133-144. https://doi.org/10.1016/ j.concog.2014.03.006

Kalin, M., Kaplan, S., Gould, F., Pinkham, A. E., Penn, D. L. y Harvey, P. D. (2015). Social cognition, social competence, negative symptoms and social outcomes: Interrelationships in people with schizophrenia. Journal of psychiatric research, 68, 254260. https://doi.org/10.1016/j.jpsychires.2015.07.008

Kamel, N. and AlQahtani, F. (2019) Social Cognition in Schizophrenia: A Review Study. Open Journal of Psychiatry, 9, 81-97. doi: 10.4236/ojpsych.2019.92007.

Kane, J. M., Kishimoto, T. y Correll, C. U. (2013). Non-adherence to medication in patients with psychotic disorders: epidemiology, contributing factors and management strategies. World psychiatry: official journal of the World Psychiatric Association (WPA), 12(3), 216-226. https://doi.org/10.1002/wps.20060

Kay, S. R., Fiszbein, A. y Opler, L. A. (1987). The positive and negative syndrome scale (PANSS) for schizophrenia. Schizophrenia bulletin, 13(2), 261-276. https://doi.org/ $10.1093 / \mathrm{schbul} / 13.2 .261$ 
Kee, K. S., Green, M. F., Mintz, J. y Brekke, J. S. (2003). Is emotion processing a predictor of functional outcome in schizophrenia?. Schizophrenia bulletin, 29(3), 487-497. https://doi.org/10.1093/oxfordjournals.schbul.a007021

Keefe, R. S., Perkins, D. O., Gu, H., Zipursky, R. B., Christensen, B. K. y Lieberman, J. A. (2006). A longitudinal study of neurocognitive function in individuals at-risk for psychosis. Schizophrenia research, 88(1-3), 26-35. https://doi.org/10.1016/j.schres. 2006.06.041

Keeley, J. W., Gaebel, W., First, M. B., Peterson, D. L., Rebello, T., Sharan, P. y Reed, G. M. (2018). Psychotic disorder symptom rating scales: Are dichotomous or multi-point scales more clinically useful?-An ICD-11 field study. Schizophrenia research, 202, 254259. https://doi.org/10.1016/j.schres.2018.07.006

Kemp, K., Zelle, H. y Bonnie, R. J. (2015). Embedding advance directives in routine care for persons with serious mental illness: implementation challenges. Psychiatric services (Washington, D.C.), 66(1), 10-14. https://doi.org/10.1176/appi.ps. 201400276

Kern, R. S., Gold, J. M., Dickinson, D., Green, M. F., Nuechterlein, K. H., Baade, L. E., Keefe, R. S., Mesholam-Gately, R. I., Seidman, L. J., Lee, C., Sugar, C. A. y Marder, S. R. (2011). The MCCB impairment profile for schizophrenia outpatients: results from the MATRICS psychometric and standardization study. Schizophrenia research, 126(1-3), 124-131. https://doi.org/10.1016/j.schres.2010.11.008

Kingdon, D. y Turkington, D. (2019). CBT for psychosis: approaches families can use. Arlington, VA: National Alliance on Mental Health.

Klein, H. S., Kelsven, S. y Pinkham, A. E. (2018). Increased social cognitive bias in subclinical paranoia. Schizophrenia research. Cognition, 12, 74-76. https://doi.org/ 10.1016/j.scog.2018.05.002

Klippel, A., Myin-Germeys, I., Chavez-Baldini, U., Preacher, K. J., Kempton, M., Valmaggia, L., Calem, M., So, S., Beards, S., Hubbard, K., Gayer-Anderson, C., Onyejiaka, A., Wichers, M., McGuire, P., Murray, R., Garety, P., van Os, J., Wykes, T., Morgan, C. y Reininghaus, U. (2017). Modeling the Interplay Between Psychological Processes and Adverse, Stressful Contexts and Experiences in Pathways to Psychosis: An Experience Sampling Study. Schizophrenia bulletin, 43(2), 302-315. https://doi.org/10.1093/ schbul/sbw185 
Kohler, C. G., Turner, T. H., Bilker, W. B., Brensinger, C. M., Siegel, S. J., Kanes, S. J., Gur, R. E. y Gur, R. C. (2003). Facial emotion recognition in schizophrenia: intensity effects and error pattern. The American journal of psychiatry, 160(10), 1768-1774. https:// doi.org/10.1176/appi.ajp.160.10.1768

Korver-Nieberg, N., Berry, K., Meijer, C. J. y de Haan, L. (2014). Adult attachment and psychotic phenomenology in clinical and non-clinical samples: a systematic review. Psychology and psychotherapy, 87(2), 127-154. https://doi.org/10.1111/papt.12010

Kottaram, A., Johnston, L. A., Cocchi, L., Ganella, E. P., Everall, I., Pantelis, C., Kotagiri, R. y Zalesky, A. (2019). Brain network dynamics in schizophrenia: Reduced dynamism of the default mode network. Human brain mapping, 40(7), 2212-2228. https:// doi.org/10.1002/hbm.24519

Kurtz, M. M., Gagen, E., Rocha, N. B., Machado, S. y Penn, D. L. (2016). Comprehensive treatments for social cognitive deficits in schizophrenia: A critical review and effectsize analysis of controlled studies. Clinical psychology review, 43, 80-89. https:// doi.org/10.1016/j.cpr.2015.09.003

Lacasa, F. y Muela, A. (2014). Guía para la aplicación e interpretación del cuestionario de apego CaMir-R. Revista de Psicopatología Y Salud Mental Del Niño Y Del Adolescente, 24, 83-93. http://doi.org/10.13140/RG.2.2.17744.30721

Landa, Y. (2019). Cognitive behavioral therapy for psychosis (CBTp): an introductory manual for clinians. New York: MIRECC VISN 2.

Langdon, R., Still, M., Connors, M. H., Ward, P. B. y Catts, S. V. (2013). Attributional biases, paranoia, and depression in early psychosis. The British journal of clinical psychology, 52(4), 408-423. https://doi.org/10.1111/bjc.12026

Le Boutillier, C., Leamy, M., Bird, V. J., Davidson, L., Williams, J. y Slade, M. (2011). What does recovery mean in practice? A qualitative analysis of international recoveryoriented practice guidance. Psychiatric services (Washington, D.C.), 62(12), 14701476. https://doi.org/10.1176/appi.ps.001312011

Lecomte, T., Leclerc C. y Wykes, T. (2016). A Guidebook for Clinicians. Group CBT for Psychosis. New York: Oxford University Press.

Lee, T. Y., Hong, S. B., Shin, N. Y. y Kwon, J. S. (2015). Social cognitive functioning in prodromal psychosis: A meta-analysis. Schizophrenia research, 164(1-3), 28-34. https://doi.org/10.1016/j.schres.2015.02.008 
Lepage, M., Bodnar, M. y Bowie, C. R. (2014). Neurocognition: clinical and functional outcomes in schizophrenia. Canadian journal of psychiatry. Revue canadienne de psychiatrie, 59(1), 5-12. https://doi.org/10.1177/070674371405900103

Levounis, P., Arnaoutt, B. y Marienfeld, C. (2017). Motivational Interviewing for Clinical Practice. Arlington, VA: American Psychiatric Association Publishing.

Lincoln, T. M., Peter, N., Schäfer, M. y Moritz, S. (2010). From stress to paranoia: an experimental investigation of the moderating and mediating role of reasoning biases. Psychological medicine, 40(1), 169-171. https://doi.org/10.1017/ S003329170999095X

Liu, Y. C., Tang, C. C., Hung, T. T., Tsai, P. C. y Lin, M. F. (2018). The Efficacy of Metacognitive Training for Delusions in Patients With Schizophrenia: A Meta-Analysis of Randomized Controlled Trials Informs Evidence-Based Practice. Worldviews on evidence-based nursing, 15(2), 130-139. https://doi.org/10.1111/wvn.12282

López-Ilundain, J. M., Pérez-Nievas, F., Otero, M. y Mata, I. (2006). Peter's delusions inventory in Spanish general population: Internal reliability, factor structure and association with demographic variables (dimensionality of delusional ideation). Actas Españolas de Psiquiatría, 34(2), 94-104.

Lozano, V., Soriano, M. F., Aznarte, J. I., Gómez-Ariza, C. J. y Bajo, M. T. (2016). Interference control commonalities in patients with schizophrenia, bipolar disorder, and borderline personality disorder. Journal of clinical and experimental neuropsychology, 38(2), 238-250. https://doi.org/10.1080/13803395.2015.1102870

Lucena, G., Cifre, I., Castillo-Garayoa J. A. y Aragonés, E. (2015). Perfil Clínico de Apego (PCA): Elaboración de un sistema de categorías para la evaluación del apego. Aloma: Revista de Psicologia, Ciències de l'Educació i de l'Esport, 33(1).

Lüdtke, T., Kriston, L., Schröder, J., Lincoln, T. M. y Moritz, S. (2017). Negative affect and a fluctuating jumping to conclusions bias predict subsequent paranoia in daily life: An online experience sampling study. Journal of behavior therapy and experimental psychiatry, 56, 106-112. https://doi.org/10.1016/j.jbtep.2016.08.014

Ludwig, K. A., Pinkham, A. E., Harvey, P. D., Kelsven, S. y Penn, D. L. (2017). Social cognition psychometric evaluation (SCOPE) in people with early psychosis: A preliminary study. Schizophrenia research, 190, 136-143. https://doi.org/10.1016/ j.schres.2017.03.001 
Lunt, L., Bramham, J., Morris, R. G., Bullock, P. R., Selway, R. P., Xenitidis, K. y David, A. S. (2012). Prefrontal cortex dysfunction and 'Jumping to Conclusions': bias or deficit?. Journal of neuropsychology, 6(1), 65-78. https://doi.org/10.1111/j. 1748-6653.2011.02005.x

Lysaker, P. H. y Dimaggio, G. (2014). Metacognitive capacities for reflection in schizophrenia: implications for developing treatments. Schizophrenia bulletin, 40(3), 487-491. https://doi.org/10.1093/schbul/sbu038

Lysaker, P. H., Hamm, J. A., Hasson-Ohayon, I., Pattison, M. L. y Leonhardt, B. L. (2018). Promoting recovery from severe mental illness: Implications from research on metacognition and metacognitive reflection and insight therapy. World journal of psychiatry, 8(1), 1-11. https://doi.org/10.5498/wjp.v8.i1.1

Lysaker, P. H. y Hasson-Ohayon, I. (2014). Metacognition in Schizophrenia: introduction to the special issue. The Israel journal of psychiatry and related sciences, 51(1), 4-7.

Lysaker, P. H., Lancaster, R. S., Nees, M. A. y Davis, L. W. (2004). Attributional style and symptoms as predictors of social function in schizophrenia. Journal of Rehabilitation Research and Development, 41(2), 225-232. https://doi.org/10.1682/JRRD. 2004.02.0225

MaBeth, A., Gumley, A., Schwannauer, M. y Fisher, R. (2011). Attachment states of mind, mentalization, and their correlates in a first-episode psychosis sample. Psychology and psychotherapy, 84(1), 42-110. https://doi.org/10.1348/147608310X530246

Mallawaarachchi, S. R., Cotton, S. M., Anderson, J., Killackey, E. y Allott, K. A. (2019). Exploring the use of the Hinting Task in first-episode psychosis. Cognitive Neuropsychiatry, 24(1), 65-79. http://doi.org/10.1080/13546805.2019.1568864

Mancuso, F., Horan, W. P., Kern, R. S. y Green, M. F. (2011). Social cognition in psychosis: multidimensional structure, clinical correlates, and relationship with functional outcome. Schizophrenia research, 125(2-3), 143-151. https://doi.org/10.1016/ j.schres.2010.11.007

Martín-Contero, M.C., Secades-Villa, R., Aparicio-Migueza, A. y Tirapu-Ustárroz, J. (2017). Empatía en el trastorno mental grave. Revista de Neurología, 64(4), 145-52. https:// dx.doi.org/10.33588/rn.6404.2016300 
Martínez, C. y Santelices, M. P. (2005). Evaluación del Apego en el Adulto: Una Revisión. Psykhe (Santiago), 14(1), 181-191. https://dx.doi.org/10.4067/ S0718-22282005000100014

Mayer, J.D., Salovey, P. y Caruso, D. R. (2002). Mayer-Salovey-Caruso Emotional Intelligence Test (MSCEITCV2.0): User's manual. Multi-Health Systems: Toronto.

Mayer, J. D., Salovey, P., Caruso, D. R. y Sitarenios, G. (2003). Measuring emotional intelligence with the MSCEIT V2.0. Emotion (Washington, D.C.), 3(1), 97-105. https:// doi.org/10.1037/1528-3542.3.1.97

Mayseless, 0. (1996). Attachment patterns and their outcomes. Human Development, 39(4), 206-223. https://doi.org/10.1159/000278448

McDonagh, M. S., Dana, T., Selph, S., Devine, E. B., Cantor, A., Bougatsos, C., Blazina, I., Grusing, S., Fu, R., Kopelovich, S. L., Monroe-DeVita, M. y Haupt, D. W. (2017). Treatments for Schizophrenia in Adults: A Systematic Review. Agency for Healthcare Research and Quality (US).

McFarlane W. R. (2016). Family Interventions for Schizophrenia and the Psychoses: A Review. Family process, 55(3), 460-482. https://doi.org/10.1111/famp.12235

McGrath, J., Saha, S., Chant, D. y Welham, J. (2008). Schizophrenia: a concise overview of incidence, prevalence, and mortality. Epidemiologic reviews, 30, 67-76. https:// doi.org/10.1093/epirev/mxn001

McGonagle, G., Bucci, S., Varese, F., Raphael, J. y Berry, K. (2019). Is adult attachment associated with engagement with services? A systematic literature review. Journal of mental health (Abingdon, England), 1-12. Advance online publication. https:// doi.org/10.1080/09638237.2019.1608922

McLean, B. F., Mattiske, J. K. y Balzan, R. P. (2017). Association of the Jumping to Conclusions and Evidence Integration Biases With Delusions in Psychosis: A Detailed Meta-analysis. Schizophrenia bulletin, 43(2), 344-354. https://doi.org/10.1093/ schbul/sbw056

McNally, K. (2011). Definitions of schizophrenia, 1908-1987: The failed essentialism. Theory \& Psychology, 22(1), 91-113. http://doi.org/10.1177/0959354310377821

McNally, K. A. (2016). A Critical History of Schizophrenia. (Palgrave Studies in the Theory and History of Psychology). Palgrave Macmillan: UK. 
Mehta, U. M., Bhagyavathi, H. D., Thirthalli, J., Kumar, K. J. y Gangadhar, B. N. (2014). Neurocognitive predictors of social cognition in remitted schizophrenia. Psychiatry research, 219(2), 268-274. https://doi.org/10.1016/j.psychres.2014.05.055

Melero, R. y Cantero, M. (2008). Los estilos afectivos en la población española: Un cuestionario de evaluación del apego adulto. Clínica Y Salud, 19, 83-100.

Moberget, T., Alnæs, D., Kaufmann, T., Doan, N. T., Córdova-Palomera, A., Norbom, L. B., ... Westlye, L. T. (2019). Cerebellar grey matter volume is associated with cognitive function and psychopathology in adolescence. bioRxiv, 288134. https://doi.org/ 10.1101/288134 http://doi.org/10.1101/288134

Molina, P., Casonato, M., Sala, M. N. y Testa, S. (2018). The Factor Structure of the CA-MIR as Evaluated Using Confirmatory Factor Analysis. Frontiers in Psychology, 9, 190. http://doi.org/10.3389/fpsyg.2018.00190

Moncrieff, J. (2013). Hablando claro. Una introducción a los fármacos psiquiátricos. Harder: Barcelona.

Monfort-Escrig, C. y Pena-Garijo, J. (2016). Aplicación individual del programa de entrenamiento metacognitivo en la esquizofrenia: dos estudios de caso. Agora Salut, 3, 245-55.

Monfort-Escrig, C. y Pena-Garijo, J. (2020). Attributional styles and social functioning in schizophrenia. Is the learned helplessness model suitable? Clinica y Salud. Ahead of print. https://doi.org/10.5093/clysa2020a21

Morgan, V. A., Morgan, F., Galletly, C., Valuri, G., Shah, S. y Jablensky, A. (2016). Sociodemographic, clinical and childhood correlates of adult violent victimisation in a large, national survey sample of people with psychotic disorders. Social Psychiatry and Psychiatric Epidemiology, 51(2), 269-279. http://doi.org/10.1007/ s00127-015-1155-6

Moreno-Küstner, B., Martín, C. y Pastor, L. (2018). Prevalence of psychotic disorders and its association with methodological issues. A systematic review and meta-analyses. PloS one, 13(4), e0195687. https://doi.org/10.1371/journal.pone.0195687

Moritz, S., Bentall, R. P., Kolbeck, K. y Roesch-Ely, D. (2018). Monocausal attribution and its relationship with reasoning biases in schizophrenia. Schizophrenia research, 193, 77-82. https://doi.org/10.1016/j.schres.2017.06.057 
Moritz, S., Kerstan, A., Veckenstedt, R., Randjbar, S., Vitzthum, F., Schmidt, C., Heise, M. y Woodward, T. S. (2011). Further evidence for the efficacy of a metacognitive group training in schizophrenia. Behaviour research and therapy, 49(3), 151-157. https:// doi.org/10.1016/j.brat.2010.11.010

Moritz, S., Klein, J. P., Desler, T., Lill, H., Gallinat, J. y Schneider, B. C. (2017). Neurocognitive deficits in schizophrenia. Are we making mountains out of molehills?. Psychological medicine, 47(15), 2602-2612. https://doi.org/10.1017/ S0033291717000939

Moritz, S. y Lysaker, P. H. (2018). Metacognition - What did James H. Flavell really say and the implications for the conceptualization and design of metacognitive interventions. Schizophrenia Research, 201, 20-26. http://doi.org/10.1016/j.schres. 2018.06.001

Moritz, S., Scheu, F., Andreou, C., Pfueller, U., Weisbrod, M. y Roesch-Ely, D. (2016). Reasoning in psychosis: Risky but not necessarily hasty. Cognitive Neuropsychiatry, 21(2), 91-106. https://doi.org/10.1080/13546805.2015.1136611

Moritz, S., Thompson, S. y Andreou, C. (2014). Illusory Control in Schizophrenia. Journal of Experimental Psychopathology, 5(2), 113-122. http://doi.org/10.5127/jep.036113

Moritz, S., Woodward, T. S., Jelinek, L. y Klinge, R. (2008). Memory and metamemory in schizophrenia: a liberal acceptance account of psychosis. Psychological medicine, 38(6), 825-832. https://doi.org/10.1017/S0033291707002553

Moritz, S., Woodward, T. S. y Lambert, M. (2007). Under what circumstances do patients with schizophrenia jump to conclusions? A liberal acceptance account. The British journal of clinical psychology, 46(Pt 2), 127-137. https://doi.org/ 10.1348/014466506X129862

Moritz, S. y Woodward, T. S. (2005). Jumping to conclusions in delusional and nondelusional schizophrenic patients. The British journal of clinical psychology, 44(Pt 2), 193-207. https://doi.org/10.1348/014466505X35678

Moritz, S., Woodward, T. S. y Hausmann, D. (2006). Incautious reasoning as a pathogenetic factor for the development of psychotic symptoms in schizophrenia. Schizophrenia bulletin, 32(2), 327-331. https://doi.org/10.1093/schbul/sbj034

Morosini, P. L., Magliano, L., Brambilla, L., Ugolini, S. y Pioli, R. (2000). Development, reliability and acceptability of a new version of the DSM-IV Social and Occupational 
Functioning Assessment Scale (SOFAS) to assess routine social functioning. Acta psychiatrica Scandinavica, 101(4), 323-329.Morrison, A. P. (2017). A manualised treatment protocol to guide delivery of evidence-based cognitive therapy for people with distressing psychosis: learning from clinical trials. Psychosis, 9(3), 271-281. http://doi.org/10.1080/17522439.2017.1295098

Morrison, A. P. (2017). A manualised treatment protocol to guide delivery of evidencebased cognitive therapy for people with distressing psychosis: learning from clinical trials. Psychosis, 9(3), 271-281. http://doi.org/10.1080/17522439.2017.1295098

Mueser, K. T., Deavers, F., Penn, D. L. y Cassisi, J. E. (2013). Psychosocial treatments for schizophrenia. Annual review of clinical psychology, 9, 465-497. https://doi.org/ 10.1146/annurev-clinpsy-050212-185620

Mueser, K. T., Penn, D. L., Addington, J., Brunette, M. F., Gingerich, S., Glynn, S. M., Lynde, D. W., Gottlieb, J. D., Meyer-Kalos, P., McGurk, S. R., Cather, C., Saade, S., Robinson, D. G., Schooler, N. R., Rosenheck, R. A. y Kane, J. M. (2015). The NAVIGATE Program for First-Episode Psychosis: Rationale, Overview, and Description of Psychosocial Components. Psychiatric services (Washington, D.C.), 66(7), 680-690. https://doi.org/ 10.1176/appi.ps.201400413

Müller, D. R. y Roder, V. (2010). Integrated psychological therapy and integrated neurocognitive therapy. In: Roder, V. y Medalia, A., editors. Neurocognition and Social Cognition in Schizophrenia Patients. Basic Concepts and Treatment. Basel: Karger, 2010. pp. 118-44.

Murphy, P., Bentall, R., Freeman, D., O'Rourke, S. y Hutton, P. (2018). The paranoia as defence model of persecutory delusions: A systematic review and meta-analysis. Lancet Psychiatry, 5(11), 913-929. https://doi.org/10.1016/ S2215-0366(18)30339-0

Nuechterlein, K. H., Barch, D. M., Gold, J. M., Goldberg, T. E., Green, M. F. y Heaton, R. K. (2004). Identification of separable cognitive factors in schizophrenia. Schizophrenia research, 72(1), 29-39. https://doi.org/10.1016/j.schres.2004.09.007

Ochoa, S., Haro, J. M., Huerta-Ramos, E., Cuevas-Esteban, J., Stephan-Otto, C., Usall, J., Nieto, L. y Brebion, G. (2014). Relation between jumping to conclusions and cognitive functioning in people with schizophrenia in contrast with healthy participants. 
Schizophrenia research, 159(1), 211-217. https://doi.org/10.1016/j.schres. 2014.07.026

Ochsner K. N. (2008). The social-emotional processing stream: five core constructs and their translational potential for schizophrenia and beyond. Biological psychiatry, 64(1), 48-61. https://doi.org/10.1016/j.biopsych.2008.04.024

Ojeda, N., Peña, J., Bengoetxea, E., Guerrero, A., Sanchez, P., Elizagárate, E., ... Eguíluz, J. (2012). [Evidence of the effectiveness of cognitive rehabilitation in psychosis and schizophrenia with the REHACOP programme]. Revista de Neurologia, 54(10), 577586.

Ojeda, N., Peña, J., Bengoetxea, E., Guerrero, A., Sanchez, P., Elizagárate, E., ... Eguíluz, J. (2012). [REHACOP: a cognitive rehabilitation programme in psychosis]. Revista de Neurologia, 54(6), 337-342.

Overall, J. E. y Gorham, D. R. (1962). The Brief Psychiatric Rating Scale. Psychological Reports, 10(3), 799-812. http://doi.org/10.2466/pr0.1962.10.3.799

Palomares, N., Portella, M. J., Díaz-Marsá, M. y López-Micó, C. (2019). Perfil neuropsicológico en primeros episodios de esquizofrenia y trastorno límite de la personalidad: un estudio comparativo. Actas españolas de Psiquiatría, 47(1), 7-15. Disponibe en: https://www.actaspsiquiatria.es/repositorio/21/117/ESP/21-117ESP-7-15-472287.pdf

Pena-Garijo, J. y Monfort-Escrig, C. (2020a). Cognición en la esquizofrenia. Estado actual de la cuestión (I): métodos de evaluación y correlatos neurales. Revista Asociación Española de Neuropsiquiatría, 40, 109-130. Retrieved from http:// www.revistaaen.es/index.php/aen/article/view/17121

Pena-Garijo, J. y Monfort-Escrig, C. (2020b). Cognición en la esquizofrenia. Estado actual de la cuestión (II): sesgos cognitivos, modelos explicativos y programas de intervención. Revista Asociación Española de Neuropsiquiatría, 40, 131-154. Retrieved from http://www.revistaaen.es/index.php/aen/article/view/17122

Pena-Garijo, J., \& Monfort-Escrig, C. (2021). The centrality of secure attachment within an interacting network of symptoms, cognition, and attachment dimensions in persons with schizophrenia-spectrum disorders: A preliminary study. Journal of Psychiatric Research. In press. https://doi.org/10.1016/j.jpsychires.2021.01.002 
Penadés, R., Catalán, R., Puig, O., Masana, G., Pujol, N., Navarro, V., Guarch, J. y Gastó, C. (2010). Executive function needs to be targeted to improve social functioning with Cognitive Remediation Therapy (CRT) in schizophrenia. Psychiatry research, 177(1-2), 41-45. https://doi.org/10.1016/j.psychres.2009.01.032

Penadés, R., Catalán, R., Pujol, N., Masana, G., García-Rizo, C. y Bernardo, M. (2012). The integration of cognitive remediation therapy into the whole psychosocial rehabilitation process: an evidence-based and person-centered approach. Rehabilitation research and practice, 2012, 386895. https://doi.org/ $10.1155 / 2012 / 386895$

Penn, D. L., Roberts, D. L., Combs, D. y Sterne, A. (2007). Best practices: The development of the Social Cognition and Interaction Training program for schizophrenia spectrum disorders. Psychiatric services (Washington, D.C.), 58(4), 449-451. https://doi.org/ 10.1176/ps.2007.58.4.449

Penn, D. L., Sanna, L. J. y Roberts, D. L. (2008). Social cognition in schizophrenia: an overview. Schizophrenia bulletin, 34(3), 408-411. https://doi.org/10.1093/schbul/ $\operatorname{sbn} 014$

Peralta, V. y Cuesta, M. J. (1994). Validación de la escala de los síndromes positivo y negativo (PANSS) en una muestra de esquizofrénicos españoles. Actas Luso-Españolas de Neurología y Psiquiatría, 22(4), 44-50.

Peralta, V. y Cuesta, M. J. (1999). Diagnostic significance of Schneider's first-rank symptoms in schizophrenia. Comparative study between schizophrenic and nonschizophrenic psychotic disorders. The British journal of psychiatry: the journal of mental science, 174, 243-248. https://doi.org/10.1192/bjp.174.3.243

Pérez-Álvarez, M. (2012). Esquizofrenia y cultura moderna: Razones de la locura. Psicothema, 24(1), 1-9.

Peters, E., Joseph, S., Day, S. y Garety, P. (2004). Measuring delusional ideation: the 21item Peters et al. Delusions Inventory (PDI). Schizophrenia bulletin, 30(4), 10051022. https://doi.org/10.1093/oxfordjournals.schbul.a007116

Peterson, C., Semmel, A., von Baeyer, C., Abramson, L. Y., Metalsky, G. I. y Seligman, M. E. P. (1982). The attributional Style Questionnaire. Cognitive Therapy and Research, 6, 287-299. https://doi.org/10.1007/BF01173577 
Peyroux, E., Prost, Z., Danset-Alexandre, C., Brenugat-Herne, L., Carteau-Martin, I., Gaudelus, B., ... Franck, N. (2019). From "under" to "over" social cognition in schizophrenia: Is there distinct profiles of impairments according to negative and positive symptoms? Schizophrenia Research. Cognition, 15, 21-29. http://doi.org/ 10.1016/j.scog.2018.10.001

Pickup, G. J. y Frith, C. D. (2001). Theory of mind impairments in schizophrenia: symptomatology, severity and specificity. Psychological medicine, 31(2), 207-220. https://doi.org/10.1017/s0033291701003385

Pierrehumbert, B., Karmaniola, A., Sieye, A., Meister, C., Miljkovitch, R. y Halfon, 0. (1996). Les modèles de relations: Développement d'un autoquestionnaire d'attachement pour adultes. Psychiatrie de l'Enfant (Vol. 39).

Pilton, M., Bucci, S., McManus, J., Hayward, M., Emsley, R. y Berry, K. (2016). Does insecure attachment mediate the relationship between trauma and voice-hearing in psychosis?. Psychiatry research, 246, 776-782. https://doi.org/10.1016/j.psychres. 2016.10.050

Pinkham, A. E., Harvey, P. D. y Penn, D. L. (2018). Social Cognition Psychometric Evaluation: Results of the Final Validation Study. Schizophrenia bulletin, 44(4), 737748. https://doi.org/10.1093/schbul/sbx117

Pinkham, A. E., Penn, D. L., Green, M. F., Buck, B., Healey, K. y Harvey, P. D. (2014). The social cognition psychometric evaluation study: results of the expert survey and RAND panel. Schizophrenia bulletin, 40(4), 813-823. https://doi.org/10.1093/ schbul/sbt081

Pino, O., Guilera, G., Rojo, J. E., Gómez-Benito, J., Bernardo, M., Crespo-Facorro, B. ... Rejas, J. (2008). Spanish version of the Screen for Cognitive Impairment in Psychiatry (SCIPS): Psychometric properties of a brief scale for cognitive evaluation in schizophrenia. Schizophrenia Research, 99, 139-148. https://doi.org/10.1016/j.schres.2007.09.012

Ponizovsky, A. M., Vitenberg, E., Baumgarten-Katz, I. y Grinshpoon, A. (2013). Attachment styles and affect regulation among outpatients with schizophrenia: relationships to symptomatology and emotional distress. Psychology and psychotherapy, 86(2), 164-182. https://doi.org/10.1111/j.2044-8341.2011.02054.x

Popolo, R., Smith, E., Lysaker, P. H., Lestingi, K., Cavallo, F., Melchiorre, L., Santone, C. y Dimaggio, G. (2017). Metacognitive profiles in schizophrenia and bipolar disorder: 
Comparisons with healthy controls and correlations with negative symptoms. Psychiatry research, 257, 45-50. https://doi.org/10.1016/j.psychres.2017.07.022

Popovic, D., Schmitt, A., Kaurani, L., Senner, F., Papiol, S., Malchow, B., Fischer, A., Schulze, T. G., Koutsouleris, N. y Falkai, P. (2019). Childhood Trauma in Schizophrenia: Current Findings and Research Perspectives. Frontiers in neuroscience, 13, 274. https:// doi.org/10.3389/fnins.2019.00274

Prike, T., Arnold, M. M. y Williamson, P. (2018). The relationship between anomalistic belief and biases of evidence integration and jumping to conclusions. Acta Psychologica, 190, 217-227. http://doi.org/10.1016/j.actpsy.2018.08.006

Quijada, Y. (2012). Impact on treatment outcome in the early stages of psychosis (Tesis doctoral). Universitat Autònoma de Barcelona.

Randjbar, S., Veckenstedt, R., Vitzthum, F., Hottenrott, B. y Moritz, S. (2011). Attributional biases in paranoid schizophrenia: Further evidence for a decreased sense of selfcausation in paranoia. Psychosis, 3(1), 74-85. http://doi.org/ $10.1080 / 17522431003717675$

Randjbar, S., Veckenstedt, R., Vitzthum, F., Hottenrott, B. y Moritz, S. (2011). Attributional biases in paranoid schizophrenia: Further evidence for a decreased sense of selfcausation in paranoia. Psychosis, 3(1), 74-85. http://doi.org/ $10.1080 / 17522431003717675$

Rajkumar, R. P. (2014). Childhood attachment and schizophrenia: The "attachmentdevelopmental-cognitive" (ADC) hypothesis. Medical Hypotheses, 83(3), 276-281. https://doi.org/10.1016/j.mehy.2014.05.017

Read, J., Bentall, R. P. y Fosse, R. (2009). Time to abandon the bio-bio-bio model of psychosis: Exploring the epigenetic and psychological mechanisms by which adverse life events lead to psychotic symptoms. Epidemiologia e psichiatria sociale, 18(4), 299-310.

Read J. (2020). Bad things happen and can drive you crazy: The causal beliefs of 701 people taking antipsychotics. Psychiatry research, 285, 112754. Advance online publication. https://doi.org/10.1016/j.psychres.2020.112754

Rebolleda, C. (2017). Déficits neurocognitivos y en cognición social en pacientes esquizofrénicos con un nivel preservado de funcionamiento intelectual general. Informaciones psiquiátricas, 227:9-23. 
Reed, G. M., First, M. B., Kogan, C. S., Hyman, S. E., Gureje, O., Gaebel, W., Maj, M., Stein, D. J., Maercker, A., Tyrer, P., Claudino, A., Garralda, E., Salvador-Carulla, L., Ray, R., Saunders, J. B., Dua, T., Poznyak, V., Medina-Mora, M. E., Pike, K. M., Ayuso-Mateos, J. L., ... Saxena, S. (2019). Innovations and changes in the ICD-11 classification of mental, behavioural and neurodevelopmental disorders. World psychiatry : official journal of the World Psychiatric Association (WPA), 18(1), 3-19. https://doi.org/10.1002/wps. 20611

Rezansoff, S. N., Moniruzzaman, A., Fazel, S., McCandless, L., Procyshyn, R. y Somers, J. M. (2017). Housing First Improves Adherence to Antipsychotic Medication Among Formerly Homeless Adults With Schizophrenia: Results of a Randomized Controlled Trial. Schizophrenia bulletin, 43(4), 852-861. https://doi.org/10.1093/schbul/ sbw136

Rocha, N. B. y Queirós, C. (2013). Metacognitive and social cognition training (MSCT) in schizophrenia: a preliminary efficacy study. Schizophrenia research, 150(1), 64-68. https://doi.org/10.1016/j.schres.2013.07.057

Roder, V. y Medalia, A. (2010). Neurocognition and Social Cognition in Schizophrenia Patients: Basic Concepts and Treatment. Karger: Basel, Switzerland.

Rodriguez-Jimenez, R., Bagney, A., Garcia-Navarro, C., Aparicio, A. I., Lopez-Anton, R., Moreno-Ortega, M., Jimenez-Arriero, M. A., Santos, J. L., Lobo, A., Kern, R. S., Green, M. F., Nuechterlein, K. H. y Palomo, T. (2012). The MATRICS consensus cognitive battery (MCCB): co-norming and standardization in Spain. Schizophrenia research, 134(2-3), 279-284. https://doi.org/10.1016/j.schres.2011.11.026

Rodríguez-Jiménez, R., Bagney, A., Moreno-Ortega, M., García-Navarro, C., Aparicio, A. I., López-Antón, R., de la Oliva, J., Jiménez-Arriero, M. A., Santos, J. L., Lobo, A. y Palomo, T. (2012). Déficit cognitivo en la esquizofrenia: MATRICS Consensus Cognitive Battery. Revista de Neurología, 55(9):549-55. https://dx.doi.org/10.33588/rn. 5509.2012246

Roisman, G. I., Holland, A., Fortuna, K., Fraley, C., Clausell, E. y Clarke, A. (2007). The Adult Attachment Interview and self-reports of attach- ment style: An empirical rapprochement. Journal of Personality and Social Psychology, 92, 678-697.

Rokita, K. I., Dauvermann, M. R. y Donohoe, G. (2018). Early life experiences and social cognition in major psychiatric disorders: A systematic review. European psychiatry: 
the journal of the Association of European Psychiatrists, 53, 123-133. https://doi.org/ 10.1016/j.eurpsy.2018.06.006

Rominger, C., Bleier, A., Fitz, W., Marksteiner, J., Fink, A., Papousek, I. y Weiss, E. M. (2016). Auditory top-down control and affective theory of mind in schizophrenia with and without hallucinations. Schizophrenia research, 174(1-3), 192-196. https:// doi.org/10.1016/j.schres.2016.05.006

Rosseel, Y. (2012). lavaan: An R Package for Structural Equation Modeling. Journal of Statistical Software, 48(2), 1-36. http://dx.doi.org/10.18637/jss.v048.i02

Rössler, W., Ajdacic-Gross, V., Müller, M., Rodgers, S., Haker, H. y Hengartner, M. P. (2015). Assessing sub-clinical psychosis phenotypes in the general population--a multidimensional approach. Schizophrenia research, 161(2-3), 194-201. https:// doi.org/10.1016/j.schres.2014.11.033

Roy, L., Crocker, A. G., Nicholls, T. L., Latimer, E. A. y Ayllon, A. R. (2014). Criminal behavior and victimization among homeless individuals with severe mental illness: a systematic review. Psychiatric services (Washington, D.C.), 65(6), 739-750. https:// doi.org/10.1176/appi.ps.201200515

Sanjuán, P. y Magallares, A. (2005). Spanish adaptation of Attributional Style Questionnaire: Psychometric properties. Trabajo presentado en la VIII European Conference of Psychological Assessment. Budapest (Hungría), 31 de Agosto - 4 de Septiembre. (Acta de Congreso: pp.: 142).

Sanjuán, P. y Magallares, A. (2006). La relación entre optimismo disposicional y estilo atribucional y su capacidad predictiva en un diseño longitudinal. Revista de Psicología General y Aplicada, 59, 71-89.

Schalinski, I., Breinlinger, S., Hirt, V., Teicher, M. H., Odenwald, M. y Rockstroh, B. (2019). Environmental adversities and psychotic symptoms: The impact of timing of trauma, abuse, and neglect. Schizophrenia research, 205, 4-9. https://doi.org/10.1016/ j.schres.2017.10.034

Schmidt, S. J., Mueller, D. R. y Roder, V. (2011). Social cognition as a mediator variable between neurocognition and functional outcome in schizophrenia: empirical review and new results by structural equation modeling. Schizophrenia bulletin, 37 Suppl 2(Suppl 2), S41-S54. https://doi.org/10.1093/schbul/sbr079 
Sergi, M. J., Green, M. F., Widmark, C., Reist, C., Erhart, S., Braff, D. L., Kee, K. S., Marder, S. R. y Mintz, J. (2007). Social cognition [corrected] and neurocognition: effects of risperidone, olanzapine, and haloperidol. The American journal of psychiatry, 164(10), 1585-1592. https://doi.org/10.1176/appi.ajp.2007.06091515

Sheffield, J. M., Rogers, B. P., Blackford, J. U., Heckers, S. y Woodward, N. D. (2019). Accelerated Aging of Functional Brain Networks Supporting Cognitive Function in Psychotic Disorders. Biological psychiatry, 86(3), 240-248. https://doi.org/10.1016/ j.biopsych.2018.12.016

Sheinbaum, T., Bedoya, E., Ros-Morente, A., Kwapil, T. y Barrantes-Vidal, N. (2013). Association between attachment prototypes and schizotypy dimensions in two independent non-clinical samples of Spanish and American young adults. Psychiatry Research, 210, 408-413. http://doi.org/10.1016/j.psychres.2013.07.020

Sheinbaum, T., Kwapil, T. R., Ballespí, S., Mitjavila, M., Chun, C. A., Silvia, P. J. y BarrantesVidal, N. (2015). Attachment style predicts affect, cognitive appraisals, and social functioning in daily life. Frontiers in psychology, 6, 296. https://doi.org/10.3389/ fpsyg.2015.00296

Shields, L. S., Pathare, S., van der Ham, A. J. y Bunders, J. (2014). A review of barriers to using psychiatric advance directives in clinical practice. Administration and policy in mental health, 41(6), 753-766. https://doi.org/10.1007/s10488-013-0523-3

Sin, J., Gillard, S., Spain, D., Cornelius, V., Chen, T. y Henderson, C. (2017). Effectiveness of psychoeducational interventions for family carers of people with psychosis: A systematic review and meta-analysis. Clinical psychology review, 56, 13-24. https:// doi.org/10.1016/j.cpr.2017.05.002

So, S. H., Tang, V. y Leung, P. W. (2015). Dimensions of Delusions and Attribution Biases along the Continuum of Psychosis. PloS one, 10(12), e0144558. https://doi.org/ 10.1371/journal.pone.0144558

Speck, G. L., Schöner, J., Bermpohl, F., Heinz, A., Gallinat, J., Majic, T. y Montag, C. (2019). Endogenous oxytocin response to film scenes of attachment and loss is pronounced in schizophrenia. Social Cognitive and Affective Neuroscience, 14(1), 109-117. https:// doi.org/10.1093/scan/nsy110

Steele, H., Steele, M. y Murphy, A. (2009). Use of the Adult Attachment Interview to measure process and change in psychotherapy. Psychotherapy research: journal of the 
Society for Psychotherapy Research, 19(6), 633-643. https://doi.org/ $10.1080 / 10503300802609698$

Stein, H., Koontz, A. D., Fonagy, P., Allen, J. G., Fultz, J., Brethour, J. R., Jr., Allen, D. y Evans, R. B. (2002). Adult attachment: What are the underlying dimensions? Psychology and Psychotherapy: Theory, Research and Practice, 75(1), 77-91. https://doi.org/ 10.1348/147608302169562

Strassnig, M., Bowie, C., Pinkham, A. E., Penn, D., Twamley, E. W., Patterson, T. L. y Harvey, P. D. (2018). Which levels of cognitive impairments and negative symptoms are related to functional deficits in schizophrenia? Journal of Psychiatric Research, 104, 124-129. http://doi.org/10.1016/j.jpsychires.2018.06.018

Szpak, M. y Białecka-Pikul, M. (2020). Links between attachment and theory of mind in childhood: Meta-analytic review. Social Development, 29(3), 653-673. https:// doi.org/10.1111/sode.12432

Takeda, T., Nakataki, M., Ohta, M., Hamatani, S., Matsuura, K. y Ohmori, T. (2018). Effect of cognitive function on jumping to conclusion in patients with schizophrenia. Schizophrenia Research. Cognition, 12, 50-55. http://doi.org/10.1016/j.scog. 2018.04.002

Tandon R. (2012). The nosology of schizophrenia: toward DSM-5 and ICD-11. The Psychiatric clinics of North America, 35(3), 557-569. https://doi.org/10.1016/j.psc. 2012.06.001

Tandon, R. y Bruijnzeel, D. (2014). The Evolving Nosology of Schizophrenia: Relevance for Treatment. En: P. G. Janicak, S. R. Marder, R. Tandon y M. Goldman (Eds). Schizophrenia: Recent Advances in Diagnosis and Treatment (pp. 13-23). New York: Springer. http://doi.org/10.1007/978-1-4939-0656-7_2

Tandon, R., Heckers, S., Bustillo, J., Barch, D. M., Gaebel, W., Gur, R. E., Malaspina, D., Owen, M. J., Schultz, S., Tsuang, M., van Os, J. y Carpenter, W. (2013). Catatonia in DSM-5. Schizophrenia research, 150(1), 26-30. https://doi.org/10.1016/j.schres. 2013.04.034

Thomas, E. C., Despeaux, K. E., Drapalski, A. L. y Bennett, M. (2018). Person-Oriented Recovery of Individuals With Serious Mental Illnesses: A Review and Meta-Analysis of Longitudinal Findings. Psychiatric services (Washington, D.C.), 69(3), 259-267. https://doi.org/10.1176/appi.ps.201700058 
Torres, A. y Olivares, J. M. (2005). Validation of the Spanish version of the social functioning scale. Actas Espanolas de Psiquiatria, 33(4), 216-220.

Trotta, A., Murray, R. M. y Fisher, H. L. (2015). The impact of childhood adversity on the persistence of psychotic symptoms: a systematic review and meta-analysis. Psychological medicine, 45(12), 2481-2498. https://doi.org/10.1017/ S0033291715000574

Turkington, D., Kingdon, D. y Weiden, P. J. (2006). Cognitive behavior therapy for schizophrenia. The American journal of psychiatry, 163(3), 365-373. https://doi.org/ 10.1176/appi.ajp.163.3.365

Üstün, Tevfik Bedirhan, Kostanjesek, N, Chatterji, S, Rehm, J \& World Health Organization. (2010). Measuring health and disability: manual for WHO Disability Assessment Schedule (WHODAS 2.0) / edited by T.B. Üstün, N. Kostanjsek, S. Chatterji, J.Rehm. World Health Organization. https://apps.who.int/iris/handle/10665/43974

Valle, R. (2020). La esquizofrenia en la CIE-11: comparación con la CIE-10 y el DSM-5. Revista de Psiquiatría Y Salud Mental, 13(2), 95-104. http://doi.org/10.1016/j.rpsm. 2020.01.001

van der Werf, M., Hanssen, M., Köhler, S., Verkaaik, M., Verhey, F. R., RISE Investigators, van Winkel, R., van Os, J. y Allardyce, J. (2014). Systematic review and collaborative recalculation of 133,693 incident cases of schizophrenia. Psychological medicine, 44(1), 9-16. https://doi.org/10.1017/S0033291712002796

van Hooren, S., Versmissen, D., Janssen, I., Myin-Germeys, I., à Campo, J., Mengelers, R., van Os, J. y Krabbendam, L. (2008). Social cognition and neurocognition as independent domains in psychosis. Schizophrenia research, 103(1-3), 257-265. https://doi.org/10.1016/j.schres.2008.02.022

van Os, J. y Linscott, R. J. (2012). Introduction: The extended psychosis phenotype-relationship with schizophrenia and with ultrahigh risk status for psychosis. Schizophrenia bulletin, 38(2), 227-230. https://doi.org/10.1093/schbul/sbr188

van Os, J. y Reininghaus, U. (2016). Psychosis as a transdiagnostic and extended phenotype in the general population. World psychiatry: official journal of the World Psychiatric Association (WPA), 15(2), 118-124. https://doi.org/10.1002/wps.20310 
van Rooijen, G., Isvoranu, A. M., Meijer, C. J., van Borkulo, C. D., Ruhe, H. G. y de Haan, L. (2017). A symptom network structure of the psychosis spectrum. Schizophrenia Research, 189, 75-83. https://doi.org/10.1016/j.schres.2017.02.018

Varese, F., Smeets, F., Drukker, M., Lieverse, R., Lataster, T., Viechtbauer, W., Read, J., van Os, J. y Bentall, R. P. (2012). Childhood adversities increase the risk of psychosis: a meta-analysis of patient-control, prospective- and cross-sectional cohort studies. Schizophrenia bulletin, 38(4), 661-671. https://doi.org/10.1093/schbul/sbs050

Varghese, D., Scott, J. y McGrath, J. (2008). Correlates of delusion-like experiences in a non-psychotic community sample. The Australian and New Zealand journal of psychiatry, 42(6), 505-508. https://doi.org/10.1080/00048670802050595

Vaskinn, A., Andersson, S., Østefjells, T., Andreassen, O. A. y Sundet, K. (2018). Emotion perception, non-social cognition and symptoms as predictors of theory of mind in schizophrenia. Comprehensive psychiatry, 85, 1-7. https://doi.org/10.1016/ j.comppsych.2018.05.002

Vaskinn, A., Løvgren, A., Egeland, M. K., Feyer, F. K., Østefjells, T., Andreassen, O. A., Melle, I. y Sundet, K. (2019). A randomized controlled trial of training of affect recognition (TAR) in schizophrenia shows lasting effects for theory of mind. European archives of psychiatry and clinical neuroscience, 269(5), 611-620. https://doi.org/10.1007/ s00406-019-00997-z

Vauth, R., Rüsch, N., Wirtz, M. y Corrigan, P. W. (2004). Does social cognition influence the relation between neurocognitive deficits and vocational functioning in schizophrenia?. Psychiatry research, 128(2), 155-165. https://doi.org/10.1016/ j.psychres.2004.05.018

Vázquez-Campo, M., Maroño, Y., Lahera, G., Mateos, R. y García-Caballero, A. (2016). eMotional Training®: Pilot study on a novel online training program on social cognition for patients with schizophrenia. Schizophrenia research. Cognition, 4, 10-17. https://doi.org/10.1016/j.scog.2015.11.007

Velligan, D. I., Weiden, P. J., Sajatovic, M., Scott, J., Carpenter, D., Ross, R. y Docherty, J. P. (2010). Strategies for addressing adherence problems in patients with serious and persistent mental illness: recommendations from the expert consensus guidelines. Journal of psychiatric practice, 16(5), 306-324. https://doi.org/10.1097/01.pra. 0000388626.98662.a0 
Ventura, J., Ered, A., Gretchen-Doorly, D., Subotnik, K. L., Horan, W. P., Hellemann, G. S. y Nuechterlein, K. H. (2015). Theory of mind in the early course of schizophrenia: stability, symptom and neurocognitive correlates, and relationship with functioning. Psychological medicine, 45(10), 2031-2043. https://doi.org/10.1017/ S0033291714003171

Vita, A., Mussoni, C., Deste, G., Ferlenghi, G., Turrina C, Valsecchi P. (2018). Psychopharmacological treatment of cognitive deficits in Schizophrenia and mood disorders. Journal of Psychopathology, 24, 62-72. https://www.jpsychopathol.it/wpcontent/uploads/2018/07/05_Vita-1.pdf

Wickham, S. y Bentall, R. (2016). Are Specific Early-Life Adversities Associated With Specific Symptoms of Psychosis?: A Patient Study Considering Just World Beliefs as a Mediator. The Journal of nervous and mental disease, 204(8), 606-613. https:// doi.org/10.1097/NMD.0000000000000511

Wickham, S., Sitko, K. y Bentall, R. P. (2015). Insecure attachment is associated with paranoia but not hallucinations in psychotic patients: the mediating role of negative self-esteem. Psychological medicine, 45(7), 1495-1507. https://doi.org/10.1017/ S0033291714002633

Wilder, C. M., Elbogen, E. B., Moser, L. L., Swanson, J. W. y Swartz, M. S. (2010). Medication preferences and adherence among individuals with severe mental illness and psychiatric advance directives. Psychiatric services (Washington, D.C.), 61(4), 380385. https://doi.org/10.1176/ps.2010.61.4.380

White, R., Haddock, G. y Varese, F. (2020). Supporting the intimate relationship needs of service users with psychosis: what are the barriers and facilitators?. Journal of mental health (Abingdon, England), 29(3), 314-320.

https://doi.org/10.1080/09638237.2019.1608928

Wojciechowska, K., Kłosińska, M., Kur, A., Wichniak, A. (2016). Neurocognition in patients with schizophrenia. Neuropoznanie u chorych na schizofrenię. Postępy Psychiatrii i Neurologii, 25, 236-47.

World Health Organization. (2018). International Classification of Diseases 11th Revision (ICD-11). World Health Organization [Internet]. https://icd.who.int/en 
Wright, J. H., Turkington, D., Kingdon, D. G. y Basco, M. R. (2009). Cognitive-Behavior Therapy for Severe Mental Illness: An Illustrated Guide. Washington, DC: American Psychiatric Publishing.

Wykes, T. (2018). Cognitive remediation - Where are we now and what should we do next? Official Journal of the Italian Society of Psychopathology, 24, 57-61.

Wykes, T., Huddy, V., Cellard, C., McGurk, S. R. y Czobor, P. (2011). A meta-analysis of cognitive remediation for schizophrenia: methodology and effect sizes. The American journal of psychiatry, 168(5), 472-485. https://doi.org/10.1176/appi.ajp. 2010.10060855

Yarnóz, S. (2008). La Teoría del Apego en la Clínica, I: Evaluación y Clínica. Madrid: Psimática.

Yárnoz Yaben, S., Alonso-Arbiol, I., Plazaola, M. y Sainz de Murieta, L. M. (2001). Apego en adultos y percepción de los otros. Anales De Psicología, 17(2), 159-170. Recuperado a partir de https://revistas.um.es/analesps/article/view/28711

Yárnoz-Yaben, S. y Comino, P. (2011). Evaluación del apego adulto: análisis de la convergencia entre diferentes instrumentos [Assessment of adult attachment: Analysis of the convergence between different instruments]. Acción Psicológica, 8, 6785. http://doi.org/10.5944/ap.8.2.191 
ANEXOS 


\section{ANEXO 1. Hoja informativa}

Univesidad Jaume I, Castellón de la Plana.

Investigadora responsable: Cristina Monfort Escrig - Director: Dr. Josep Pena Garijo

\section{INVESTIGACIÓN DOCTORAL SOBRE SESGOS COGNITIVOS}

Este estudio, en el que se le invita a participar, pertenece al proyecto de investigación doctoral sobre sesgos cognitivos en diferentes trastornos psiquiátricos como la esquizofrenia y la depresión. El estudio de estos componentes del razonamiento puede ayudar a la elaboración de intervenciones y tratamientos más específicos para la mejora de la salud psicológica.

Si usted acepta participar se someterá a una sesión de evaluación. En esta sesión se le pide que realice una serie de pruebas y cuestionarios que permiten identificar componentes relacionados con el estudio: cognición, apego, creencias y emociones.

De acuerdo con la ley 15/1999 de Protección de Datos de Carácter Personal, los datos que se le van a solicitar son los necesarios para cubrir los objetivos del estudio. En ninguno de los informes del estudio aparecerá su nombre y su identidad no será revelada a persona alguna. La información de estos datos solo será conocida por los investigadores que se comprometen a la confidencialidad y que utilizaran los resultados solo con fines científicos (los resultados del estudio pueden ser comunicados en congresos o pueden ser objeto de publicación en revistas científicas).

De acuerdo con la ley vigente, tiene usted derecho a conocer los resultados que haya obtenido en cualquiera de las pruebas que se realicen con usted, para lo cual y una vez analizados se le citará para hacérselos saber.

Su participación en este estudio es voluntaria y en cualquier momento, si lo desea, puede usted renunciar a seguir en el mismo sin que esto le suponga perjuicio alguno.

Ninguno de los investigadores recibe compensación económica por este proyecto.

Firma del investigador 


\section{ANEXO 2. Hoja de consentimiento informado}

Univesidad Jaume I, Castellón de la Plana.

Investigador responsable: Cristina Monfort Escrig - Director: Dr. Josep Pena Garijo

\section{INVESTIGACIÓN DOCTORAL SOBRE SESGOS COGNITIVOS}

\section{HOJA DE CONSENTIMIENTO INFORMADO}

He sido invitado a participar voluntariamente por parte de (el/la profesional responsable), al estudio de investigación doctoral sobre sesgos cognitivos.

He leído la hoja de información acerca de este proyecto que se me ha entregado.

He comprendido las explicaciones ofrecidas por la investigadora Cristina Monfort Escrig y he podido hacer las preguntas que he creído convenientes.

Atendiendo a todas las consideraciones anteriores

Yo D. /Dña.

con DNI:

mayor de edad, acepto participar de forma voluntaria en el mencionado estudio y comprendo que puedo retirarme cuando quiera sin tener que dar explicaciones. Así mismo me comprometo expresamente a decir la verdad en mis respuestas para garantizar la veracidad de los datos que se me solicitan.

Fecha de la aceptación:

Firma del participante

Firma del investigador

RELLENAR SÓLO EN CASO DE DECIDIR ABANDONAR DURANTE LA SESIÓN:

Debido a un cambio en mis circunstancias personales, decido revocar el anterior consentimiento y dejar de participar en el estudio sin que esto tenga ningún tipo de consecuencias negativas para mi. 
ANEXO 3. Hoja de datos

\section{ESTUDIO DE INVESTIGACIÓN SESGOS COGNITIVOS - DOSSIER DE CUESTIONARIOS}

Iniciales:

Código paciente (rellena investigador):

Fecha de hoy:

Edad: años

Sexo:
Hombre
Mujer

Nivel de estudios (indicar hasta qué curso se han completado):

Estado civil:

Soltero

Casado / Pareja

$\square$ Viudo

$\square$ Separado

Estado laboral:

$\square$ Trabaja

Labores Hogar

Paro

Pensionista

Estudiante 
Escala para el Síndrome Positivo y Negativo de la Esquizofrenia (PANSS) Adaptación espanola de Peralta y Cuesta (1994)

\begin{tabular}{|c|c|c|c|c|c|c|c|}
\hline 1. Delirios & 1 & 2 & 3 & 4 & 5 & 6 & 7 \\
\hline 2. Desorganización conceptual & 1 & 2 & 3 & 4 & 5 & 6 & 7 \\
\hline 3. Alucinaciones & 1 & 2 & 3 & 4 & 5 & 6 & 7 \\
\hline 4. Excitación & 1 & 2 & 3 & 4 & 5 & 6 & 7 \\
\hline 5. Grandiosidad & 1 & 2 & 3 & 4 & 5 & 6 & 7 \\
\hline 6. Suspicacia/perjuicio & 1 & 2 & 3 & 4 & 5 & 6 & 7 \\
\hline 7. Hostilidad & 1 & 2 & 3 & 4 & 5 & 6 & 7 \\
\hline Escala positiva (PANSS-P) & \multicolumn{2}{|c|}{ Puntuacion directa: } & \multicolumn{5}{|c|}{ Percentil: } \\
\hline 1. Embotamiento afectivo & 1 & 2 & 3 & 4 & 5 & 6 & 7 \\
\hline 2. Retraimiento emocional & 1 & 2 & 3 & 4 & 5 & 6 & 7 \\
\hline 3. Contacto pobre & 1 & 2 & 3 & 4 & 5 & 6 & 7 \\
\hline 4. Retraimiento social & 1 & 2 & 3 & 4 & 5 & 6 & 7 \\
\hline 5. Pensamiento abstracto & 1 & 2 & 3 & 4 & 5 & 6 & 7 \\
\hline 6. Fluidez de la conversación & 1 & 2 & 3 & 4 & 5 & 6 & 7 \\
\hline 7. Pensamiento estereotipado & 1 & 2 & 3 & 4 & 5 & 6 & 7 \\
\hline Escala negativa (PANSS-N) & \multicolumn{2}{|c|}{ Puntuacion directa: } & \multicolumn{5}{|c|}{ Percentil: } \\
\hline & 1 & 2 & 3 & 4 & 5 & 6 & 7 \\
\hline Escala compuesta (PANSS-C) & \multicolumn{2}{|c|}{ Puntuación directa: } & \multicolumn{5}{|c|}{ Percentil: } \\
\hline 1. Preocupación somática & 1 & 2 & 3 & 4 & 5 & 6 & 7 \\
\hline 2. Ansiedad & 1 & 2 & 3 & 4 & 5 & 6 & 7 \\
\hline 3. Sentimientos de culpa & 1 & 2 & 3 & 4 & 5 & 6 & 7 \\
\hline 4. Tensión motora & 1 & 2 & 3 & 4 & 5 & 6 & 7 \\
\hline 5. Manierismos/posturas & 1 & 2 & 3 & 4 & 5 & 6 & 7 \\
\hline 6. Depresión & 1 & 2 & 3 & 4 & 5 & 6 & 7 \\
\hline 7. Enlentecimiento motor & 1 & 2 & 3 & 4 & 5 & 6 & 7 \\
\hline 8. Falta de colaboración & 1 & 2 & 3 & 4 & 5 & 6 & 7 \\
\hline 9. Pensamientos inusuales & 1 & 2 & 3 & 4 & 5 & 6 & 7 \\
\hline 10. Desorientación & 1 & 2 & 3 & 4 & 5 & 6 & 7 \\
\hline 11. Atención deficiente & 1 & 2 & 3 & 4 & 5 & 6 & 7 \\
\hline 12. Ausencia de insight & 1 & 2 & 3 & 4 & 5 & 6 & 7 \\
\hline 13. Trastornos de volición & 1 & 2 & 3 & 4 & 5 & 6 & 7 \\
\hline 14. Control deficiente de los impulsos & 1 & 2 & 3 & 4 & 5 & 6 & 7 \\
\hline 15. Ensimismamiento & 1 & 2 & 3 & 4 & 5 & 6 & 7 \\
\hline 16. Evitación social activa & 1 & 2 & 3 & 4 & 5 & 6 & 7 \\
\hline \multirow[t]{2}{*}{ Psicopatología general (PANSS-PG) } & \multicolumn{2}{|c|}{ Puntuación directa: } & \multicolumn{5}{|c|}{ Percentil: } \\
\hline & 1 & 2 & 3 & 4 & 5 & 6 & 7 \\
\hline
\end{tabular}




\section{INVENTARIO DE IDEAS DELIRANTES DE PETERS-21 (PDI-21)}

López-llundain, Pérez-Nievas, Otero y Mata (2006)

Este cuestionario ha sido diseñado para medir la frecuencia de determinadas ideas y fenómenos psíquicos que la mayor parte de la gente ha experimentado en algún momento de su vida.

\section{Instrucciones:}

- Es importante que usted conteste a todas las preguntas.

- Cuando usted conteste NO a una pregunta, por favor pase directamente a la siguiente pregunta.

- Cuando usted conteste Sí a una pregunta, estaremos interesados en: a) cuánto le inquietan o molestan estas creencias o experiencias; $b$ ) cuánto piensa en ellas; y c) en qué medida piensa usted que estas creencias o experiencias son ciertas.

- Al final de cada pregunta usted podrá puntuar estos tres aspectos en una escala del 1 al 5 según su intensidad.

1. ¿Tiene alguna vez la sensación de que la gente insinúa cosas sobre usted o le dicen cosas con un doble sentido?

NO Sí (marque la respuesta adecuada)

Si ha contestado $\mathrm{NO} \rightarrow$ pase a la siguiente pregunta

Si ha contestado Sí $\rightarrow$ rodee las cifras que mejor describan cómo se siente:

$\begin{array}{lllllll}\text { No me inquieta en absoluto } & 1 & 2 & 3 & 4 & 5 & \text { Me inquieta muchísimo }\end{array}$

$\begin{array}{lllllll}\text { Casi nunca pienso en ello } & 1 & 2 & 3 & 4 & 5 & \text { Pienso en ello continuamente }\end{array}$

$\begin{array}{lllllll}\text { No creo que sea cierto } & 1 & 2 & 3 & 4 & 5 & \text { Creo que es totalmente cierto }\end{array}$

2. ¿Tiene alguna vez la sensación de que hay cosas que aparecen en la televisión o en el periódico dirigidas especialmente a usted?

NO sí (marque la respuesta adecuada)

Si ha contestado $\mathrm{NO} \rightarrow$ pase a la siguiente pregunta

Si ha contestado Sí $\rightarrow$ rodee las cifras que mejor describan cómo se siente:

$\begin{array}{lllllll}\text { No me inquieta en absoluto } & 1 & 2 & 3 & 4 & 5 & \text { Me inquieta muchísimo } \\ \text { Casi nunca pienso en ello } & 1 & 2 & 3 & 4 & 5 & \text { Pienso en ello continuamente } \\ \text { No creo que sea cierto } & 1 & 2 & 3 & 4 & 5 & \text { Creo que es totalmente cierto }\end{array}$

3. ¿Tiene alguna vez la sensación de que algunas personas no son realmente lo que aparentan ser?

NO Sí (marque la respuesta adecuada)

Si ha contestado $\mathrm{NO} \rightarrow$ pase a la siguiente pregunta

Si ha contestado Sí $\rightarrow$ rodee las cifras que mejor describan cómo se siente:

$\begin{array}{lllllll}\text { No me inquieta en absoluto } & 1 & 2 & 3 & 4 & 5 & \text { Me inquieta muchísimo } \\ \text { Casi nunca pienso en ello } & 1 & 2 & 3 & 4 & 5 & \text { Pienso en ello continuamente } \\ \text { No creo que sea cierto } & 1 & 2 & 3 & 4 & 5 & \text { Creo que es totalmente cierto }\end{array}$


4. ¿Tiene alguna vez la sensación de que está siendo perseguido de algún modo?

Si ha contestado $\mathrm{NO} \rightarrow$ pase a la siguiente pregunta

NO Sí (marque la respuesta adecuada)

Si ha contestado Sí $\rightarrow$ rodee las cifras que mejor describan cómo se siente:

$\begin{array}{llllllll}\text { No me inquieta en absoluto } & 1 & 2 & 3 & 4 & 5 & \text { Me inquieta muchísimo }\end{array}$

$\begin{array}{llllllll}\text { Casi nunca pienso en ello } & 1 & 2 & 3 & 4 & 5 & \text { Pienso en ello continuamente }\end{array}$

$\begin{array}{lllllll}\text { No creo que sea cierto } & 1 & 2 & 3 & 4 & 5 & \text { Creo que es totalmente cierto }\end{array}$

5. ¿Tiene alguna vez la sensación de que existe una conspiración contra usted?

NO Sí (marque la respuesta adecuada)

$\mathrm{Si}$ ha contestado $\mathrm{NO} \rightarrow$ pase a la siguiente pregunta

$\mathrm{Si}$ ha contestado Sí $\rightarrow>$ rodee las cifras que mejor describan cómo se siente:

$\begin{array}{llllllll}\text { No me inquieta en absoluto } & 1 & 2 & 3 & 4 & 5 & \text { Me inquieta muchísimo }\end{array}$

$\begin{array}{llllllll}\text { Casi nunca pienso en ello } & 1 & 2 & 3 & 4 & 5 & \text { Pienso en ello continuamente }\end{array}$

$\begin{array}{lllllll}\text { No creo que sea cierto } & 1 & 2 & 3 & 4 & 5 & \text { Creo que es totalmente cierto }\end{array}$

6. ¿Tiene alguna vez la sensación de ser una persona muy importante o de estar destinado a serlo?

NO Sí (marque la respuesta adecuada)

$\mathrm{Si}$ ha contestado $\mathrm{NO} \rightarrow$ pase a la siguiente pregunta

Si ha contestado Sí $\rightarrow>$ rodee las cifras que mejor describan cómo se siente:

$\begin{array}{lllllll}\text { Casi nunca pienso en ello } & 1 & 2 & 3 & 4 & 5 & \text { Pienso en ello continuamente }\end{array}$

$\begin{array}{lllllll}\text { No creo que sea cierto } & 1 & 2 & 3 & 4 & 5 & \text { Creo que es totalmente cierto }\end{array}$

7. ¿Tiene alguna vez la sensación de ser una persona muy especial, fuera de lo común?

NO Sí (marque la respuesta adecuada)

Si ha contestado $\mathrm{NO} \rightarrow$ pase a la siguiente pregunta

Si ha contestado Sí $\rightarrow>$ rodee las cifras que mejor describan cómo se siente:

$\begin{array}{llllllll}\text { No me inquieta en absoluto } & 1 & 2 & 3 & 4 & 5 & \text { Me inquieta muchísimo }\end{array}$

$\begin{array}{lllllll}\text { Casi nunca pienso en ello } & 1 & 2 & 3 & 4 & 5 & \text { Pienso en ello continuamente }\end{array}$

$\begin{array}{llllllll}\text { No creo que sea cierto } & 1 & 2 & 3 & 4 & 5 & \text { Creo que es totalmente cierto }\end{array}$

8. ¿Tiene alguna vez la sensación de que usted está especialmente cerca de Dios?

Si ha contestado $\mathrm{NO} \rightarrow>$ pase a la siguiente pregunta

NO Sí (marque la respuesta adecuada)

Si ha contestado Sí $\rightarrow$ rodee las cifras que mejor describan cómo se siente:

$\begin{array}{lllllll}\text { No me inquieta en absoluto } & 1 & 2 & 3 & 4 & 5 & \text { Me inquieta muchísimo } \\ \text { Casi nunca pienso en ello } & 1 & 2 & 3 & 4 & 5 & \text { Pienso en ello continuamente } \\ \text { No creo que sea cierto } & 1 & 2 & 3 & 4 & 5 & \text { Creo que es totalmente cierto }\end{array}$


9. ¿Ha llegado usted a pensar que la gente se puede comunicar por telepatía?

NO Sí (marque la respuesta adecuada)

Si ha contestado $\mathrm{NO} \rightarrow$ pase a la siguiente pregunta

Si ha contestado Sí $\rightarrow>$ rodee las cifras que mejor describan cómo se siente:

$\begin{array}{llllllll}\text { No me inquieta en absoluto } & 1 & 2 & 3 & 4 & 5 & \text { Me inquieta muchísimo }\end{array}$

$\begin{array}{llllllll}\text { Casi nunca pienso en ello } & 1 & 2 & 3 & 4 & 5 & \text { Pienso en ello continuamente }\end{array}$

$\begin{array}{lllllll}\text { No creo que sea cierto } & 1 & 2 & 3 & 4 & 5 & \text { Creo que es totalmente cierto }\end{array}$

10. ¿Tiene alguna vez la sensación de algunos aparatos eléctricos, como los ordenadores, pueden influenciar a distancia su forma de pensar?

$\mathrm{Si}$ ha contestado $\mathrm{NO} \rightarrow$ pase a la siguiente pregunta

NO Sí (marque la respuesta adecuada)

Si ha contestado Sí $\rightarrow>$ rodee las cifras que mejor describan cómo se siente:

$\begin{array}{llllllll}\text { No me inquieta en absoluto } & 1 & 2 & 3 & 4 & 5 & \text { Me inquieta muchísimo }\end{array}$

$\begin{array}{llllllll}\text { Casi nunca pienso en ello } & 1 & 2 & 3 & 4 & 5 & \text { Pienso en ello continuamente }\end{array}$

$\begin{array}{llllllll}\text { No creo que sea cierto } & 1 & 2 & 3 & 4 & 5 & \text { Creo que es totalmente cierto }\end{array}$

11. ¿Tiene alguna vez la sensación de que usted ha sido, de algún modo, elegido por Dios?

NO Sí (marque la respuesta adecuada)

$\mathrm{Si}$ ha contestado $\mathrm{NO} \rightarrow$ pase a la siguiente pregunta

Si ha contestado Sí $\rightarrow>$ rodee las cifras que mejor describan cómo se siente:

$\begin{array}{llllllll}\text { No me inquieta en absoluto } & 1 & 2 & 3 & 4 & 5 & \text { Me inquieta muchísimo }\end{array}$

$\begin{array}{lllllll}\text { Casi nunca pienso en ello } & 1 & 2 & 3 & 4 & 5 & \text { Pienso en ello continuamente }\end{array}$

$\begin{array}{lllllll}\text { No creo que sea cierto } & 1 & 2 & 3 & 4 & 5 & \text { Creo que es totalmente cierto }\end{array}$

12. ¿Cree usted en el poder de la brujería, del vudú y de las fuerzas ocultas?

NO Sí (marque la respuesta adecuada)

$\mathrm{Si}$ ha contestado $\mathrm{NO} \rightarrow$ pase a la siguiente pregunta

Si ha contestado Sí $\rightarrow>$ rodee las cifras que mejor describan cómo se siente:

No me inquieta en absoluto

Casi nunca pienso en ello

$\begin{array}{lllll}1 & 2 & 3 & 4 & 5\end{array}$

Pienso en ello continuamente

No creo que sea cierto

$\begin{array}{lllll}1 & 2 & 3 & 4 & 5\end{array}$

Creo que es totalmente cierto

13. ¿Está usted a menudo preocupado porque su pareja le pueda ser infiel?

NO Sí (marque la respuesta adecuada)

$\mathrm{Si}$ ha contestado $\mathrm{NO} \rightarrow$ pase a la siguiente pregunta

Si ha contestado Sí $\rightarrow>$ rodee las cifras que mejor describan cómo se siente:

$\begin{array}{llllllll}\text { No me inquieta en absoluto } & 1 & 2 & 3 & 4 & 5 & \text { Me inquieta muchísimo }\end{array}$

$\begin{array}{llllllll}\text { Casi nunca pienso en ello } & 1 & 2 & 3 & 4 & 5 & \text { Pienso en ello continuamente }\end{array}$

$\begin{array}{llllllll}\text { No creo que sea cierto } & 1 & 2 & 3 & 4 & 5 & \text { Creo que es totalmente cierto }\end{array}$ 
14. ¿Tiene alguna vez la sensación de que usted ha cometido más pecados que la mayoría de la gente?

NO Sí (marque la respuesta adecuada)

$\mathrm{Si}$ ha contestado $\mathrm{NO} \rightarrow>$ pase a la siguiente pregunta

Si ha contestado Sí $\rightarrow$ rodee las cifras que mejor describan cómo se siente:

$\begin{array}{llllllll}\text { No me inquieta en absoluto } & 1 & 2 & 3 & 4 & 5 & \text { Me inquieta muchísimo }\end{array}$

$\begin{array}{llllllll}\text { Casi nunca pienso en ello } & 1 & 2 & 3 & 4 & 5 & \text { Pienso en ello continuamente }\end{array}$

$\begin{array}{llllllll}\text { No creo que sea cierto } & 1 & 2 & 3 & 4 & 5 & \text { Creo que es totalmente cierto }\end{array}$

15. ¿Tiene alguna vez la sensación de que la gente le mira de forma extraña por su aspecto o apariencia?

NO Sí (marque la respuesta adecuada)

Si ha contestado $\mathrm{NO} \rightarrow$ pase a la siguiente pregunta

Si ha contestado Sí $\rightarrow>$ rodee las cifras que mejor describan cómo se siente:

$\begin{array}{llllllll}\text { No me inquieta en absoluto } & 1 & 2 & 3 & 4 & 5 & \text { Me inquieta muchísimo }\end{array}$

$\begin{array}{llllllll}\text { Casi nunca pienso en ello } & 1 & 2 & 3 & 4 & 5 & \text { Pienso en ello continuamente }\end{array}$

$\begin{array}{llllllll}\text { No creo que sea cierto } & 1 & 2 & 3 & 4 & 5 & \text { Creo que es totalmente cierto }\end{array}$

16. ¿Tiene alguna vez la sensación de que no tiene ningún pensamiento en su cabeza?

NO Sí (marque la respuesta adecuada)

$\mathrm{Si}$ ha contestado $\mathrm{NO} \rightarrow$ pase a la siguiente pregunta

Si ha contestado Sí $\rightarrow>$ rodee las cifras que mejor describan cómo se siente:

No me inquieta en absoluto

$12-3$

$45 \quad$ Me inquieta muchísimo

Casi nunca pienso en ello

$\begin{array}{lllll}1 & 2 & 3 & 4 & 5\end{array}$

Pienso en ello continuamente

No creo que sea cierto

$\begin{array}{lllll}1 & 2 & 3 & 4 & 5\end{array}$

Creo que es totalmente cierto

17. ¿Tiene alguna vez la sensación de que el mundo está a punto de terminar?

NO Sí (marque la respuesta adecuada)

$\mathrm{Si}$ ha contestado $\mathrm{NO} \rightarrow>$ pase a la siguiente pregunta

Si ha contestado Sí $\rightarrow>$ rodee las cifras que mejor describan cómo se siente:

\begin{tabular}{|c|c|c|c|c|c|c|}
\hline No me inquieta en absoluto & 1 & 2 & 3 & 4 & 5 & Me inquieta muchísimo \\
\hline Casi nunca pienso en ello & 1 & $?$ & 3 & 4 & 5 & Pienso en ello continuamente \\
\hline No creo que sea cierto & 1 & 2 & 3 & 4 & 5 & Creo que es totalmente cierto \\
\hline \multicolumn{7}{|c|}{$\begin{array}{l}\text { 18. ¿Tiene alguna vez la sensación de tener pensamientos en su cabeza que usted no reconoce como } \\
\text { propios? }\end{array}$} \\
\hline \multicolumn{7}{|c|}{$\begin{array}{l}\text { Si ha contestado NO } \rightarrow \text { p pase a la siguiente pregunta } \\
\text { Si ha contestado Sí } \rightarrow>\text { rodee las cifras que mejor describan cómo se siente: }\end{array}$} \\
\hline No me inquieta en absoluto & 1 & 2 & 3 & 4 & 5 & Me inquieta muchísimo \\
\hline Casi nunca pienso en ello & 1 & 2 & 3 & 4 & 5 & Pienso en ello continuamente \\
\hline No creo que sea cierto & 1 & 2 & 3 & 4 & 5 & Creo que es totalmente cierto \\
\hline
\end{tabular}


19. ¿Han sido alguna vez sus pensamientos tan intensamente vividos que le ha llegado a preocupar el que otras personas le pudieran oír?

NO Sí (marque la respuesta adecuada)

Si ha contestado $\mathrm{NO} \rightarrow$ pase a la siguiente pregunta

Si ha contestado Sí $\rightarrow>$ rodee las cifras que mejor describan cómo se siente:

$\begin{array}{llllllll}\text { No me inquieta en absoluto } & 1 & 2 & 3 & 4 & 5 & \text { Me inquieta muchísimo }\end{array}$

$\begin{array}{llllllll}\text { Casi nunca pienso en ello } & 1 & 2 & 3 & 4 & 5 & \text { Pienso en ello continuamente }\end{array}$

$\begin{array}{lllllll}\text { No creo que sea cierto } & 1 & 2 & 3 & 4 & 5 & \text { Creo que es totalmente cierto }\end{array}$

20. ¿Tiene alguna vez la sensación de oír sus propios pensamientos repetidos como por un eco?

NO Sí (marque la respuesta adecuada)

Si ha contestado $\mathrm{NO} \rightarrow$ pase a la siguiente pregunta

Si ha contestado Sí $\rightarrow>$ rodee las cifras que mejor describan cómo se siente:

$\begin{array}{llllllll}\text { No me inquieta en absoluto } & 1 & 2 & 3 & 4 & 5 & \text { Me inquieta muchísimo }\end{array}$

$\begin{array}{lllllll}\text { Casi nunca pienso en ello } & 1 & 2 & 3 & 4 & 5 & \text { Pienso en ello continuamente }\end{array}$

$\begin{array}{llllllll}\text { No creo que sea cierto } & 1 & 2 & 3 & 4 & 5 & \text { Creo que es totalmente cierto }\end{array}$

21. ¿Tiene alguna vez la sensación de ser como un robot o un zombi, como si su cuerpo no obedeciera a su propia voluntad?

$\mathrm{Si}$ ha contestado $\mathrm{NO} \rightarrow$ pase a la siguiente pregunta

Si ha contestado Sí $\rightarrow>$ rodee las cifras que mejor describan cómo se siente:

$\begin{array}{llllllll}\text { No me inquieta en absoluto } & 1 & 2 & 3 & 4 & 5 & \text { Me inquieta muchísimo }\end{array}$

$\begin{array}{llllllll}\text { Casi nunca pienso en ello } & 1 & 2 & 3 & 4 & 5 & \text { Pienso en ello continuamente }\end{array}$

$\begin{array}{lllllll}\text { No creo que sea cierto } & 1 & 2 & 3 & 4 & 5 & \text { Creo que es totalmente cierto }\end{array}$ 


\section{ANEXO 6. BDI-II}

\section{INVENTARIO DE DEPRESIÓN DE BECK (BDI-II)}

Adaptación española de Sanz, Perdigón y Vázquez (2003)

Instrucciones: Este cuestionario consta de 21 grupos de afirmaciones. Por favor, lea con atención cada uno de ellos cuidadosamente. Luego elija uno de cada grupo, el que mejor describa el modo como se ha sentido las últimas dos semanas, incluyendo el día de hoy. Marque con un círculo el número correspondiente al enunciado elegido. $\mathrm{Si}$ varios enunciados de un mismo grupo le parecen igualmente apropiados, marque el número más alto. Verifique que no haya elegido más de uno por grupo, incluyendo el ítem 16 (cambios en los hábitos de sueño) y el ítem 18 (cambios en el apetito).

\section{Tristeza}

0 No me siento triste.

1 Me siento triste gran parte del tiempo.

2 Me siento triste todo el tiempo.

3 Me siento tan triste o soy tan infeliz que no puedo soportarlo.

\section{Pesimismo}

0 No estoy desalentado respecto a mi futuro.

1 Me siento más desalentado respecto de mi futuro que lo que solía estarlo.

2 No espero que las cosas funcionen para mi.

3 Siento que no hay esperanza para mi futuro y que sólo puede empeorar.

\section{Fracaso}

0 No me siento como un fracasado.

1 He fracasado más de lo que hubiera debido.

2 Cuando miro hacia atrás, veo muchos fracasos.

3 Siento que como persona soy un fracaso total.

\section{Pérdida de placer}

0 Obtengo tanto placer como siempre por las cosas de las que disfruto.

1 No disfruto tanto de las cosas como solía hacerlo.

2 Obtengo muy poco placer de las cosas que solía disfrutar.

3 No puedo obtener ningún placer de las cosas de las que solía disfrutar.

\section{Sentimientos de culpa}

0 No me siento particularmente culpable.

$1 \mathrm{Me}$ siento culpable respecto de varias cosas que he hecho o que debería haber hecho.

2 Me siento bastante culpable la mayor parte del tiempo.

3 Me siento culpable todo el tiempo. 


\section{Sentimientos de castigo}

0 No siento que este siendo castigado

1 Siento que tal vez pueda ser castigado.

2 Espero ser castigado.

3 Siento que estoy siendo castigado.

\section{Disconformidad con uno mismo}

0 Siento acerca de mi lo mismo que siempre.

1 He perdido la confianza en mí mismo.

2 Estoy decepcionado conmigo mismo.

3 No me gusto a mí mismo.

\section{Autocrítica}

0 No me critico ni me culpo más de lo habitual.

1 Estoy más crítico conmigo mismo de lo que solía estarlo.

2 Me critico a mí mismo por todos mis errores.

3 Me culpo a mí mismo por todo lo malo que sucede.

\section{Pensamientos o deseos suicidas}

0 No tengo ningún pensamiento de matarme.

1 He tenido pensamientos de matarme, pero no lo haría.

2 Querría matarme.

3 Me mataría si tuviera la oportunidad de hacerlo.

\section{Llanto}

0 No lloro más de lo que solía hacerlo.

1 Lloro más de lo que solía hacerlo.

2 Lloro por cualquier pequeñez.

3 Siento ganas de llorar pero no puedo.

\section{Agitación}

0 No estoy más inquieto o tenso que lo habitual.

1 Me siento más inquieto o tenso que lo habitual.

2 Estoy tan inquieto o agitado que me es difícil quedarme quieto.

3 Estoy tan inquieto o agitado que tengo que estar siempre en movimiento o haciendo algo.

\section{Pérdida de interés}

0 No he perdido el interés en otras actividades o personas.

1 Estoy menos interesado que antes en otras personas o cosas.

2 He perdido casi todo el interés en otras personas o cosas.

$3 \mathrm{Me}$ es difícil interesarme por algo. 


\section{Indecisión}

0 Tomo mis propias decisiones tan bien como siempre.

1 Me resulta más difícil que de costumbre tomar decisiones

2 Encuentro mucha más dificultad que antes para tomar decisiones.

3 Tengo problemas para tomar cualquier decisión.

\section{Desvalorización}

0 No siento que yo no sea valioso.

1 No me considero a mi mismo tan valioso y útil como solía considerarme.

2 Me siento menos valioso cuando me comparo con otros.

3 Siento que no valgo nada.

\section{Pérdida de energía}

0 Tengo tanta energía como siempre.

1. Tengo menos energía que la que solía tener.

2. No tengo suficiente energía para hacer demasiado

3. No tengo energía suficiente para hacer nada.

\section{Cambios en los hábitos de sueño}

0 No he experimentado ningún cambio en mis hábitos de sueño.

1a Duermo un poco más que lo habitual.

$1 b$ Duermo un poco menos que lo habitual.

2a Duermo mucho más que lo habitual.

$2 b$ Duermo mucho menos que lo habitual.

3a Duermo la mayor parte del día.

$3 b$ Me despierto 1-2 horas más temprano y no puedo volver a dormirme.

\section{Irritabilidad}

0 No estoy tan irritable que lo habitual.

1 Estoy más irritable que lo habitual.

2 Estoy mucho más irritable que lo habitual.

3 Estoy irritable todo el tiempo. 


\section{Cambios en el apetito}

0 No he experimentado ningún cambio en mi apetito.

1a Mi apetito es un poco menor que lo habitual.

$1 b \mathrm{Mi}$ apetito es un poco mayor que lo habitual.

2a Mi apetito es mucho menor que antes.

$2 b \mathrm{Mi}$ apetito es mucho mayor que lo habitual

3a No tengo apetito en absoluto.

$3 b$ Quiero comer todo el día.

\section{Dificultad de concentración}

0 Puedo concentrarme tan bien como siempre.

1 No puedo concentrarme tan bien como habitualmente.

2 Me es difícil mantener la mente en algo por mucho tiempo.

3 Encuentro que no puedo concentrarme en nada.

\section{Cansancio o fatiga}

0 No estoy más cansado o fatigado que lo habitual.

1 Me fatigo o me canso más fácilmente que lo habitual.

2 Estoy demasiado fatigado o cansado para hacer muchas de las cosas que solía hacer.

3 Estoy demasiado fatigado o cansado para hacer la mayoría de las cosas que solía hacer.

\section{Pérdida de interés en el sexo}

0 No he notado ningún cambio reciente en mi interés por el sexo.

1Estoy menos interesado en el sexo de lo que solía estarlo.

2 Estoy mucho menos interesado en el sexo.

3 He perdido completamente el interés en el sexo.

\section{Puntuación total:}




\section{ESCALA REDUCIDA DE FUNCIONAMIENTO SOCIAL (SFS)}

Alonso, J. y cols. (2008)

\section{Aislamiento - Actividades sociales}

\begin{tabular}{ll} 
¿Con qué frecuencia sale de casa? (por cualquier razón) & $0=$ Nunca \\
& $1=$ Raramente \\
2 & $=$ A veces \\
& $3=$ A menudo \\
\hline ¿Cómo reacciona ante la presencia de extraños? & $0=$ Los evita \\
$1=$ Se siente nervioso \\
$2=$ Los acepta \\
$3=$ Le agradan
\end{tabular}

\section{Comunicación interpersonal}

$\begin{array}{ll}\text { En estos momentos, ¿le resulta fácil o difícil hablar con la } & 3=\text { Bastante fácil } \\ \text { gente? } & 2=\text { Normal } \\ 1=\text { Bastante difícil } \\ 0=\text { Muy difícil }\end{array}$

Conducta prosocial: marque en cada columna la frecuencia con la que ha participado en cualquiera de las siguiente actividades durante los últimos 3 meses.

\begin{tabular}{|ll}
\hline Exposición & $0=$ Nunca \\
& $1=$ Raramente \\
2 & $=$ A veces \\
& $3=$ A menudo \\
\hline Ir a fiestas & $0=$ Nunca \\
& $1=$ Raramente \\
2 & $=$ A veces \\
& $3=$ A menudo \\
Discoteca & $0=$ Nunca \\
& $1=$ Raramente \\
& $2=$ A veces \\
$3=$ A menudo \\
Asistir a alguna actividad de la iglesia & $0=$ Nunca \\
& $1=$ Raramente \\
$2=$ A veces \\
$3=$ A menudo
\end{tabular}

Independencia - Ejecución

Marque en cada ítem la frecuencia con que ha realizado las siguientes actividades durante los últimos 3 meses.

\begin{tabular}{|ll}
\hline Lavar la propia ropa & $0=$ Nunca \\
& $1=$ Raramente \\
& $2=$ A veces \\
& $3=$ A menudo \\
& $0=$ Nunca \\
& $1=$ Raramente \\
Utilizar dinero & $2=$ A veces \\
$3=$ A menudo \\
\hline
\end{tabular}




\begin{tabular}{|ll}
\hline Disponer y administrar el propio dinero & $0=$ Nunca \\
& $1=$ Raramente \\
$2=$ A veces \\
$3=$ A menudo
\end{tabular}

\section{Independencia - Competencia: decir si es capaz de hacer}

\begin{tabular}{|c|c|}
\hline Cocinar para sí mismo & $\begin{array}{l}3=\text { Adecuadamente }, \text { no necesita ayuda } \\
2=\text { Necesita ayuda o que le estimulen } \\
1=\text { Incapaz } . \text { Necesita mucha ayuda } \\
0=\text { No sabe }\end{array}$ \\
\hline Fregar los platos, etc. & $\begin{array}{l}3=\text { Adecuadamente }, \text { no necesita ayuda } \\
2=\text { Necesita ayuda } \text { o que le estimulen } \\
1=\text { Incapaz } . \text { Necesita mucha ayuda } \\
0=\text { No sabe }\end{array}$ \\
\hline
\end{tabular}

Actividades del tiempo libre: marque donde corresponda en cada columna para indicar con qué frecuencia ha hecho alguna de las siguientes actividades durante los últimos 3 meses.

Arreglar cosas (bici, coche, etc.)

$$
\begin{aligned}
& 0=\text { Nunca } \\
& 1=\text { Raramente } \\
& 2=\text { A veces } \\
& 3=\text { A menudo }
\end{aligned}
$$

\section{Empleo - Ocupación}

¿Tiene usted en estos momentos un empleo regular? (Incluye terapia ocupacional, rehabilitación o cursos de reciclaje)

¿Está usted dado de bajo como incapacitado?

$$
1 \text { = Sí }
$$

$2=$ No

$$
1 \text { = Sí }
$$$$
2=\text { No }
$$ 


\section{ANEXO 8. CAMIR-R}

\section{CAMIR-R}

Balluerka, N., Lacasa, F. (2011)

Este cuestionario trata sobre las ideas y sentimientos que tienes de tus relaciones personales y familiares. Tanto del presente, como de tu infancia. Lee cada frase y rodea el número de la opción que mejor describa tus ideas y sentimientos.

\begin{tabular}{|c|c|c|c|c|c|c|}
\hline & & $\begin{array}{l}\text { Muy de } \\
\text { acuerdo }\end{array}$ & $\begin{array}{l}\text { De } \\
\text { acuerdo }\end{array}$ & Indeciso & $\begin{array}{l}\text { En } \\
\text { desacuer } \\
\text { do }\end{array}$ & $\begin{array}{l}\text { Muy en } \\
\text { desacuer } \\
\text { do }\end{array}$ \\
\hline 1 & $\begin{array}{l}\text { Las amenazas de separación, de traslado a } \\
\text { otro lugar, o de ruptura de los lazos familiares } \\
\text { son parte de mis recuerdos infantiles. }\end{array}$ & 5 & 4 & 3 & 2 & 1 \\
\hline 2 & $\begin{array}{l}\text { Mis padres eran incapaces de tener autoridad } \\
\text { cuando era necesario. }\end{array}$ & 5 & 4 & 3 & 2 & 1 \\
\hline 3 & $\begin{array}{l}\text { En caso de necesidad, estoy seguro(a) de que } \\
\text { puedo contar con mis seres queridos para } \\
\text { encontrar consuelo. }\end{array}$ & 5 & 4 & 3 & 2 & 1 \\
\hline 4 & $\begin{array}{l}\text { Desearía que mis hijos fueran más autónomos } \\
\text { de los que yo lo he sido. }\end{array}$ & 5 & 4 & 3 & 2 & 1 \\
\hline 5 & $\begin{array}{l}\text { En la vida de familia, el respeto a los padres } \\
\text { es muy importante. }\end{array}$ & 5 & 4 & 3 & 2 & 1 \\
\hline 6 & $\begin{array}{l}\text { Cuando yo era niño(a), sabía que siempre } \\
\text { encontraría consuelo en mis seres queridos. }\end{array}$ & 5 & 4 & 3 & 2 & 1 \\
\hline 7 & $\begin{array}{l}\text { Las relaciones con mis eres queridos durante } \\
\text { mi niñez, me parecen, en general, positivas. }\end{array}$ & 5 & 4 & 3 & 2 & 1 \\
\hline 8 & $\begin{array}{l}\text { Detesto el sentimiento de depender de los } \\
\text { demás. }\end{array}$ & 5 & 4 & 3 & 2 & 1 \\
\hline 9 & $\begin{array}{l}\text { Sólo cuento conmigo mismo para resolver mis } \\
\text { problemas. }\end{array}$ & 5 & 4 & 3 & 2 & 1 \\
\hline 10 & $\begin{array}{l}\text { Cuando yo era niño(a), a menudo, mis seres } \\
\text { queridos se mostraban impacientes e } \\
\text { irritables. }\end{array}$ & 5 & 4 & 3 & 2 & 1 \\
\hline 11 & $\begin{array}{l}\text { Mis seres queridos siempre me han dado lo } \\
\text { mejor de sí mismos. }\end{array}$ & 5 & 4 & 3 & 2 & 1 \\
\hline 12 & $\begin{array}{l}\text { No puedo concentrarme sobre otra cosa, } \\
\text { sabiendo que alguno de mis seres queridos } \\
\text { tiene problemas. }\end{array}$ & 5 & 4 & 3 & 2 & 1 \\
\hline 13 & $\begin{array}{l}\text { Cuando yo era niño(a), encontré suficiente } \\
\text { cariño en mis seres queridos como para no } \\
\text { buscarlo en otra parte. }\end{array}$ & 5 & 4 & 3 & 2 & 1 \\
\hline 14 & $\begin{array}{l}\text { Siempre estoy preocupado(a) por la pena que } \\
\text { puedo causar a mis seres queridos al dejarlos. }\end{array}$ & 5 & 4 & 3 & 2 & 1 \\
\hline 15 & $\begin{array}{l}\text { Cuando era niño(a), tenían una actitud de } \\
\text { dejarme hacer. }\end{array}$ & 5 & 4 & 3 & 2 & 1 \\
\hline
\end{tabular}




\begin{tabular}{|c|c|c|c|c|c|c|}
\hline & & $\begin{array}{l}\text { Muy de } \\
\text { acuerdo }\end{array}$ & $\begin{array}{l}\text { De } \\
\text { acuerdo }\end{array}$ & Indeciso & $\begin{array}{l}\text { En } \\
\text { desacuer } \\
\text { do }\end{array}$ & $\begin{array}{l}\text { Muy en } \\
\text { desacuer } \\
\text { do }\end{array}$ \\
\hline 16 & $\begin{array}{l}\text { De adolescente, nadie de mi entorno entendía } \\
\text { del todo mis preocupaciones. }\end{array}$ & 5 & 4 & 3 & 2 & 1 \\
\hline 17 & $\begin{array}{l}\text { Cuando yo era niño(a), teníamos mucha } \\
\text { dificultad para tomar decisiones en familia. }\end{array}$ & 5 & 4 & 3 & 2 & 1 \\
\hline 18 & $\begin{array}{l}\text { Tengo la sensación de que nunca superaría la } \\
\text { muerte de uno de mis seres queridos. }\end{array}$ & 5 & 4 & 3 & 2 & 1 \\
\hline 19 & $\begin{array}{l}\text { Los niños deben sentir que existe una } \\
\text { autoridad respetada dentro de la familia. }\end{array}$ & 5 & 4 & 3 & 2 & 1 \\
\hline 20 & $\begin{array}{l}\text { Mis padres no se han dado cuento que un } \\
\text { niño(a) cuando crece tiene necesidad de tener } \\
\text { vida propia. }\end{array}$ & 5 & 4 & 3 & 2 & 1 \\
\hline 21 & Siento confianza en mis seres queridos. & 5 & 4 & 3 & 2 & 1 \\
\hline 22 & $\begin{array}{l}\text { Mis padre me han dado demasiada libertad } \\
\text { para hacer todo lo que yo quería. }\end{array}$ & 5 & 4 & 3 & 2 & 1 \\
\hline 23 & $\begin{array}{l}\text { Cuando yo era niño(a), tuve que enfrentarme a } \\
\text { la violencia de uno de mis seres queridos. }\end{array}$ & 5 & 4 & 3 & 2 & 1 \\
\hline 24 & $\begin{array}{l}\text { A partir de mi experiencia de niño(a), he } \\
\text { compre ndido que nunca som os } \\
\text { suficientemente buenos para los padres. }\end{array}$ & 5 & 4 & 3 & 2 & 1 \\
\hline 25 & $\begin{array}{l}\text { Cuando yo era niño(a), se preocuparon tanto } \\
\text { por mi salud y mi seguridad, que me sentía } \\
\text { aprisionado(a). }\end{array}$ & 5 & 4 & 3 & 2 & 1 \\
\hline 26 & $\begin{array}{l}\text { Cuando me alejo de mis seres queridos, no } \\
\text { me siento bien conmigo mismo. }\end{array}$ & 5 & 4 & 3 & 2 & 1 \\
\hline 27 & $\begin{array}{l}\text { Mis padres no podía evitar controlarlo todo: mi } \\
\text { apariencia, mis resultados escolares e incluso } \\
\text { mis amigos. }\end{array}$ & 5 & 4 & 3 & 2 & 1 \\
\hline 28 & $\begin{array}{l}\text { Cuando era niño(a), había peleas } \\
\text { insoportables en casa. }\end{array}$ & 5 & 4 & 3 & 2 & 1 \\
\hline 29 & Es importante que el niño aprende a obedecer. & 5 & 4 & 3 & 2 & 1 \\
\hline 30 & $\begin{array}{l}\text { Cuando yo era niño(a), mis seres queridos me } \\
\text { hacian sentir que les gustaba compartir su } \\
\text { tiempo conmigo. }\end{array}$ & 5 & 4 & 3 & 2 & 1 \\
\hline 31 & $\begin{array}{l}\text { La idea de separación momentánea con uno } \\
\text { de mis seres queridos, me deja una sensación } \\
\text { de inquietud. }\end{array}$ & 5 & 4 & 3 & 2 & 1 \\
\hline 32 & $\begin{array}{l}\text { A menudo, me siento preocupado(a), sin } \\
\text { razón, por la salud de mis seres queridos. }\end{array}$ & 5 & 4 & 3 & 2 & 1 \\
\hline
\end{tabular}


ANEXO 9. ASQ

CUESTIONARIO SOBRE EL ESTILO DE ATRIBUCIONES (ASQ)

Peterson, Semmel, Seligman y cols. (1982)

Instrucciones:

1. Lea cada situación e image lo más vívidamente posible que le está sucediendo a usted.

2. Cuál cree usted que es la causa más importante de la situación en caso que le suceda a usted.

3. Escriba esta causa en los espacios reservados para ello.

4. Responda a las tres preguntas acerca de la causa, en una escala del 1 al 7 , haciendo un círculo en un número por pregunta. No haga círculo en las palabras.

5. Pase a la situación siguiente.

Situación 1: Se encuentra con un amigo(a) que le da cumplidos o lo felicita por como se lo ve, por la forma en que luce.

1. Escriba la causa más importante de este cumplido:

2. La causa del cumplido que le dio su amigo(a), ¿es debido a algo acerca de usted o a algo acerca de otra gente o de otras circunstancias?

Totalmente debido a otra gente 1 23 34 5 6 Totalmente debido o circunstancias

3. En el futuro, cuando esté con su amigo(a), ¿estará presente esta misma causa otra vez?
Nunca estará
1
23
4
56
7
Siempre estará presente otra vez presente

4. La causa, ¿es algo que sólo afecta o influye en la relación con amigos(as), o es algo que influye también otras áreas de su vida?

$\begin{array}{clllllllll}\begin{array}{l}\text { Influye sólo esta } \\ \text { situación particular }\end{array} & 1 & 2 & 3 & 4 & 5 & 6 & 7 & \begin{array}{c}\text { Influye en todas las } \\ \text { situaciones en mi } \\ \text { vida }\end{array}\end{array}$


1. Escriba la causa más importante:

2. La causa de la falta de resultados positivos ¿es debida a algo acerca de usted o a algo debido a otra gente o circunstancias?

Totalmente debido a otra gente

$$
\begin{array}{llllllll}
1 & 2 & 3 & 4 & 5 & 6 & 7
\end{array}
$$

Totalmente debido o circunstancias

3. En el futuro, cuando vuelva a buscar trabajo, ¿estará presente esta misma causa otra vez?
Nunca estará
1
23
$\begin{array}{lllll}3 & 4 & 5 & 6 & 7\end{array}$ presente otra vez

4. Esta causa, ¿solamente influye en buscar trabajo o también influencia en otras áreas de su vida? Influye sólo esta situación particular $\begin{array}{llllllll}1 & 2 & 3 & 4 & 5 & 6 & 7\end{array}$ Influye en todas las situaciones en mi vida

\section{Situación 3: Tiene mucho éxito y buenos resultados.}

1. Escriba la causa más importante de esto:

2. La causa de que tenga éxito en lo que está emprendiendo, ¿es debido a algo acerca de usted o a algo acerca de otra gente o de otras circunstancias?

Totalmente debido a otra gente o circunstancias

3. En el futuro, ¿estima que esta causa estará presente otra vez?
Nunca estará
12
3
4
56
7

Totalmente debido a mí

4. Esta causa, ¿es algo que solamente influye en que pueda tener buenos logros o éxitos (dinero o empleo o estudios) o también influencia en otras áreas de su vida?

Influye sólo esta situación particular

1

$\begin{array}{lllllll}2 & 3 & 4 & 5 & 6 & 7\end{array}$

Influye en todas las situaciones en mi vida 
1. Escriba la causa más importante:

2. La causa de que usted no lo ayude, ¿es debido a algo acerca de usted o a algo acerca de otra gente o de otras circunstancias?

Totalmente debido a otra gente

$\begin{array}{lllllll}1 & 2 & 3 & 4 & 5 & 6 & 7\end{array}$

Totalmente debido o circunstancias

3. En el futuro, cuando un amigo acuda a usted con un problema, ¿esta causa estará otra vez presente?
Nunca estará
1
23
$4 \quad 5$
6
7
Siempre estará presente otra vez presente

4. La causa, ¿es algo que solamente afecta o influye cuando un amigo(a) acude a usted con un problema, o también influencia otras áreas de su vida?

Influye sólo esta situación particular $\begin{array}{llll}1 & 2 & 3 & 4\end{array}$
Influye en todas las situaciones en mi vida

Situación 5: Da una importante charla frente a un grupo y la audiencia reacciona negativamente hacia usted.

1. Escriba cuál considera que es la causa más importante de esto:

2. La causa de la reacción negativa de la audiencia, ¿es algo referido a usted o es algo relacionado con otras personas o circunstancias?

Totalmente debido a otra gente

o circunstancias

3. En el futuro, cuando de una charla otra vez ¿esta causa estará otra vez presente?

\section{Nunca estará} presente otra vez
4. La causa, ¿es algo que solamente influye en dar charlas o hablar frente a un grupo, o también influencia en otras áreas de su vida?

Influye sólo esta situación particular

$\begin{array}{lllllll}1 & 2 & 3 & 4 & 5 & 6 & 7\end{array}$

$\begin{array}{lllllll}1 & 2 & 3 & 4 & 5 & 6 & 7\end{array}$

\section{(1)}

Siempre estará presente 


\section{Situación 6: Usted hace un proyecto que es altamente valorado.}

1. Escriba cuál es la causa más importante de esta valoración:

2. La causa de que sea apreciado y valorado, ¿es debido a algo relativo a usted o a algo acerca de otra gente o circunstancias?

Totalmente debido a otra gente

$\begin{array}{lllllll}1 & 2 & 3 & 4 & 5 & 6 & 7\end{array}$

Totalmente debido o circunstancias

3. En el futuro, cuando elabore un proyecto nuevamente, ¿estará la causa presente otra vez?
Nunca estará
12
3
45
$6 \quad 7$
Siempre estará presente otra vez

4. La causa, ¿solamente influye o afecta el hacer proyectos, o también influencia otras áreas de su vida? Influye sólo esta situación particular

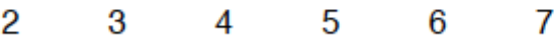

Influye en todas las situaciones en mi vida

\section{Situación 7: Se encuentra con un amigo(a) que actúa hostilmente hacia usted.}

1. Escriba la causa que considera más importante:

2. La causa de que su amigo(a) actúe hostil, ¿es debido a algo acerca de usted, o a algo debido a otra gente o circunstancias?

$\begin{array}{cccccccccc}\begin{array}{c}\text { Totalmente } \\ \text { debido a otra gente }\end{array} & 1 & 2 & 3 & 4 & 5 & 6 & 7 & \text { Totalmente debido } \\ \text { a mí }\end{array}$

3. En el futuro, cuando interactúe con amigos(as), ¿esta causa estará presente otra vez?

$\begin{array}{cllllllll}\begin{array}{c}\text { Nunca estará } \\ \text { presente otra vez }\end{array} & 1 & 2 & 3 & 4 & 5 & 6 & 7 & \begin{array}{c}\text { Siempre estará } \\ \text { presente }\end{array}\end{array}$

4. La causa, ¿es algo que que solamente influye en la interacción y relación con los amigos(as), o también influye en otras áreas de su vida?

$\begin{array}{clllllllll}\begin{array}{l}\text { Influye sólo esta } \\ \text { situación particular }\end{array} & 1 & 2 & 3 & 4 & 5 & 6 & 7 & \begin{array}{c}\text { Influye en todas las } \\ \text { situaciones en mi } \\ \text { vida }\end{array}\end{array}$


1. Escriba cuál es la causa que considera más importante:

2. La causa de no lograr el nivel esperado del trabajo, ¿es algo relativo a usted, o más bien tiene que ver con otra gente o circunstancias?

Totalmente debido a otra gente

1

$\begin{array}{llllll}2 & 3 & 4 & 5 & 6 & 7\end{array}$

Totalmente debido o circunstancias

3. Cuando en el futuro tenga que hacer un trabajo en el que la gente le pone expectativas, ¿estará esta causa presente otra vez?
Nunca estará
1
23
45
67
presente otra vez

4. La causa, ¿es algo que solamente influye el hacer el trabajo que los demás esperan de usted, o también influencia otras áreas de su vida?

$\begin{array}{clllllllll}\begin{array}{c}\text { Influye sólo esta } \\ \text { situación particular }\end{array} & 1 & 2 & 3 & 4 & 5 & 6 & 7 & \begin{array}{c}\text { Influye en todas las } \\ \text { situaciones en mi } \\ \text { vida }\end{array}\end{array}$

\section{Situación 9: Su cónyuge o novio(a) le ha estado tratando con amor y consideración.}

1. Escriba cuál considera es la causa más importante de esto:

2. El que su pareja le esté tratando más amorosamente, ¿es debido a razones relacionadas con usted o es algo relacionado con otra gente $\mathrm{u}$ otras circunstancias?

$\begin{array}{cccccccccc}\begin{array}{c}\text { Totalmente } \\ \text { debido a otra gente }\end{array} & 1 & 2 & 3 & 4 & 5 & 6 & 7 & \text { Totalmente debido } \\ \text { a mí }\end{array}$

3. En las futuras interacciones con su pareja, ¿estará presente esta causa otra vez?

$\begin{array}{cllllllll}\begin{array}{c}\text { Nunca estará } \\ \text { presente otra vez }\end{array} & 1 & 2 & 3 & 4 & 5 & 6 & 7 & \begin{array}{c}\text { Siempre estará } \\ \text { presente }\end{array}\end{array}$

4. La causa, ¿es algo que que solamente afecta o influye en cómo le trata su pareja, o es algo que influye en otras áreas de su vida?

Influye sólo esta situación particular

$\begin{array}{llllllll}1 & 2 & 3 & 4 & 5 & 6 & 7\end{array}$

Influye en todas las situaciones en mi vida 
Situación 10: Aspira ingresar a una actividad que valora, como por ejemplo, un trabajo importante o una admisión en una buena universidad. Postula y al final lo obtiene.

1. Escriba la causa que considere más importante para este logro:

2. ¿Es la causa de obtener esta posición debido a usted o es debido a otras personas o circunstancias?

Totalmente

debido a otra gente

$\begin{array}{lllllll}1 & 2 & 3 & 4 & 5 & 6 & 7\end{array}$

Totalmente debido

o circunstancias

3. En el futuro, cuando trate de lograr otra posición importante, ¿esta causa estará presente otra vez?

$\begin{array}{cccccccccc}\begin{array}{c}\text { Nunca estará } \\ \text { presente otra vez }\end{array} & 1 & 2 & 3 & 4 & 5 & 6 & 7 & \begin{array}{c}\text { Siempre estará } \\ \text { presente }\end{array}\end{array}$

4. Esta causa, ¿es algo que que solamente influye para aspirar a una posición y lograrla, o también influye en otras áreas de su vida?

$\begin{array}{lllllllll}\begin{array}{l}\text { Influye sólo esta } \\ \text { situación particular }\end{array} & 1 & 2 & 3 & 4 & 5 & 6 & 7 & \begin{array}{c}\text { Influye en todas las } \\ \text { situaciones en mi } \\ \text { vida }\end{array}\end{array}$

\section{Situación 11: Sale con alguna o algunas personas (cita, reunión social) y siente que le fue mal.}

1. Escriba cuál considera que es la causa más importante para esto:

2. ¿Es la causa de que le vaya mal en este encuentro algo que se debe a usted, o es algo debido a otra gente o circunstancias?

Totalmente debido a otra gente

1

$\begin{array}{llllll}2 & 3 & 4 & 5 & 6 & 7\end{array}$

Totalmente debido

o circunstancias

3. En el futuro, cuando tenga otros encuentros, ¿estará esta causa otra vez presente?
Nunca estará
1
23
45
6
7
presente otra vez

4. ¿Es la causa algo que solamente influye en estos encuentros, o es algo que también influye en otras áreas de su vida?

Influye sólo esta

situación particular

Influye en todas las situaciones en mi vida 
Situación 12: Se le mejoran las condiciones laborales o de situación económica.

1. Escriba cuál estima que es la causa más importante:

2. ¿Es la causa de la mejoría algo debido a usted o es algo debido a otra gente o circunstancias?

Totalmente debido a otra gente

$\begin{array}{lllllll}1 & 2 & 3 & 4 & 5 & 6 & 7\end{array}$

Totalmente debido o circunstancias

3. En el futuro, en su trabajo o actividades, ¿esta causa estará presente nuevamente?
Nunca estará
12
3
34
Siempre estará presente otra vez presente

4. ¿Es esta causa algo que solamente afecta el obtener un mejoramiento en las condiciones laborales o influencia también otras áreas de su vida?

Influye sólo esta situación particular

$\begin{array}{lllllll}1 & 2 & 3 & 4 & 5 & 6 & 7\end{array}$

Influye en todas las situaciones en mi vida 


\section{Test de las Insinuaciones - Adaptación española de la Hinting Task}

(Gil, Fernández-Modamio, Bengochea y Arrieta, 2012)

\section{A.1. Instrucciones}

Voy a leerle un total de 10 historias en las que aparecen dos personas. Cada historia termina con uno de los personajes diciendo algo. Cuando haya terminado de leer las historias, voy a hacerle algunas preguntas sobre lo que dijo el personaje.

\begin{tabular}{|l|l|l|}
\hline Historia & Respuesta literal 1 y puntuación & Respuesta literal 2 y puntuación \\
\hline 1-Viaje largo y caluroso & & \\
\hline 2-Baño sucio & & \\
\hline 3-Caramelos & & \\
\hline 4-Camisa arrugada & & \\
\hline 5-jSin un duro! & & \\
\hline 6-Proyecto de trabajo & & \\
\hline 7-Regalo de cumpleaños & & \\
\hline 8-Adornos & & \\
\hline 9-Juego de trenes & & \\
\hline 10-Maletas pesadas & & \\
\hline
\end{tabular}

La puntuación en cada historia puede ser de 0, 1 o 2 / La puntuación total de las 10 historias va de 0 a 20

\section{A.2. Puntuación}

\section{A.2.1. HISTORIA 1}

Jorge llega a la oficina de Ángela después de un largo y caluroso viaje por la autopista. Ángela inmediatamente empieza a hablar de algunas ideas de negocios. Jorge interrumpe a Ángela diciendo:

"Uf, ha sido un largo y caluroso viaje por la autopista".

PREGUNTA: ¿Qué quiere decir realmente Jorge cuando dice esto?

A.2.1.1. Respuesta literal. Respuesta criterio: Jorge quiere decir “ ¿Puedo tomar algo?» o « ¿Puedo tomarme unos minutos para descansar de mi viaje antes de que empecemos a hablar de negocios?". Esta respuesta se puntuaría con un 2 y se pasaría a la historia siguiente.

Si no se da una respuesta correcta con la primera insinuación (por ejemplo, el sujeto responde tan solo algo como "Él quiere decir exactamente lo que dice»), entonces se introduce la siguiente parte de la insinuación:

AÑADIR: Jorge continúa diciendo: «Estoy sediento».

PREGUNTA: ¿Qué quiere Jorge que haga Ángela? 


\section{A.2.2. HISTORIA 2}

Marisa va a cuarto de baño para darse una ducha. Ana acaba de darse un baño. Marisa se da cuenta de que el cuarto de baño está sucio, así que llama a Ana y le dice:

“¿No pudiste encontrar el limpiador, Ana?».

PREGUNTA: ¿Qué quiere decir realmente Marisa cuando dice esto?

1.1.1.1. Respuesta literal. Respuesta criterio: Marisa quiere decir « ¿Por qué no limpiaste el baño?" o "Ve y limpia el baño ahora". Esta respuesta se puntuaría con un 2 y se pasaría a la historia siguiente.

$\mathrm{Si}$ el sujeto no da la respuesta correcta, AÑADIR: Marisa continúa diciendo: «A veces eres muy vaga, Ana".

PREGUNTA: ¿Qué quiere Marisa que haga Ana?

1.1.1.2. Respuesta literal. Respuesta criterio: Marisa quiere que Ana limpie el baño. Esta respuesta puntuaría 1. Cualquier otra respuesta sería un 0.

\section{A.2.3. HISTORIA 3}

Javier va al supermercado con su madre. Llegan a la sección de chucherías y Javier dice: "Mira, esos caramelos parecen riquísimos".

PREGUNTA: ¿Qué quiere decir realmente Javier cuando dice esto?

A.2.3.1. Respuesta literal. Respuesta criterio: Javier quiere decir «Por favor, mamá, cómprame algunos caramelos». Esta respuesta se puntuaría con un 2 y se pasaría a la historia siguiente.

Si el sujeto no da la respuesta correcta, AÑADIR: Javier continúa diciendo: «Estoy hambriento mamá».

PREGUNTA: ¿Qué quiere Javier que haga su madre?

A.2.3.2. Respuesta literal. Respuesta criterio: Javier quiere que su madre le compre unos caramelos. Esta respuesta puntuaría 1. Cualquier otra respuesta sería un 0.

\section{A.2.4. HISTORIA 4}

Pablo tiene que ir a una entrevista y se le está haciendo tarde. Mientras está limpiándose los zapatos, le dice a su mujer, Julia:

"Quiero ponerme esa camisa azul, pero está muy arrugada".

PREGUNTA: ¿Qué quiere decir realmente Pablo cuando dice esto?

A.2.4.1. Respuesta literal. Respuesta criterio: Pablo quiere decir "¿Podrías plancharme la camisa, por favor?". Esta respuesta se puntuaría con un 2 y se pasaría a la historia siguiente.

Si el sujeto no da la respuesta correcta, AÑADIR: Pablo continúa diciendo: «Está en la cesta de la ropa para planchar».

PREGUNTA: ¿Qué quiere Pablo que haga Julia?

A.2.4.2. Respuesta literal. Respuesta criterio: Pablo quiere que Julia le planche la camisa. Esta respuesta puntuaría 1. Cualquier otra respuesta sería un 0. 


\section{A.2.5. HISTORIA 5}

Lucía está sin un duro, pero quiere salir por la noche. Lucía sabe que David acaba de cobrar. Ella le dice:

"Estoy sin un duro, las cosas son tan caras en estos tiempos".

PREGUNTA: ¿Qué quiere decir realmente Lucía cuando dice esto?

A.2.5.1. Respuesta literal. Respuesta criterio: Lucía quiere decir “¿Me prestas algo de dinero, David?» o "¿Me invitas a salir esta noche?». Esta respuesta se puntuaría con un 2 y se pasaría a la historia siguiente.

Si el sujeto no da la respuesta correcta, AÑADIR: Lucía continúa diciendo: «Bien, supongo que tendré que quedarme sin salir esta noche".

PREGUNTA: ¿Qué quiere Lucía que haga David?

A.2.5.2. Respuesta literal. Respuesta criterio: Lucía quiere que David le preste dinero, o la invite a salir. Esta respuesta puntuaría 1. Cualquier otra respuesta sería un 0.

\section{A.2.6. HISTORIA 6}

Daniel quiere llevar a cabo un proyecto en su trabajo pero Ricardo, su jefe, ha pedido a otra persona que lo realice. Daniel le dice:

"Qué lástima, no estoy demasiado ocupado en este momento".

PREGUNTA: ¿Qué quiere decir realmente Daniel cuando dice esto?

A.2.6.1. Respuesta literal. Respuesta criterio: Daniel quiere decir «Por favor, Ricardo, cambia de opinión y dame el proyecto a mí». Esta respuesta se puntuaría con un 2 y se pasaría a la historia siguiente.

Si el sujeto no da la respuesta correcta, AÑADIR: Daniel continúa diciendo: «Este proyecto es ideal para mí».

PREGUNTA: ¿Qué quiere Daniel que haga Ricardo?

A.2.6.2. Respuesta literal. Respuesta criterio: Daniel quiere que Ricardo cambie de opinión y le dé a él el proyecto. Esta respuesta puntuaría 1. Cualquier otra respuesta sería un 0 .

\section{A.2.7. HISTORIA 7}

Dentro de poco es el cumpleaños de Rebeca. Ella le dice a su padre:

"Me encantan los animales, sobre todo, los perros".

PREGUNTA: ¿Qué quiere decir realmente Rebeca cuando dice esto?

A.2.7.1. Respuesta literal. Respuesta criterio: Rebeca quiere decir "¿Me comprarías un perro para mi cumpleaños, papá?». Esta respuesta se puntuaría con un 2 y se pasaría a la historia siguiente.

Si el sujeto no da la respuesta correcta, AÑADIR: Rebeca continúa diciendo: «¿La tienda de mascotas estará abierta el día de mi cumpleaños, papá?».

PREGUNTA: ¿Qué quiere Rebeca que haga su padre?

A.2.7.2. Respuesta literal. Respuesta criterio: Rebeca quiere que su padre le compre un perro para su cumpleaños, o que le diga que le comprará un perro. Esta respuesta puntuaría 1. Cualquier otra respuesta sería un 0. 


\section{A.2.8. HISTORIA 8}

Beatriz y Miguel se han mudado a su nueva casa hace una semana. Beatriz ha estado desempaquetando algunos adornos. Ella le dice a Miguel:

"¿Has desempaquetado aquellas estanterías que compramos, Miguel?».

PREGUNTA: ¿Qué quiere decir realmente Beatriz cuando dice esto?

A.2.8.1. Respuesta literal. Respuesta criterio: Beatriz quiere decir "¿Pondrías las estanterías ahora, por favor?». Esta respuesta se puntuaría con un 2 y se pasaría a la historia siguiente.

Si el sujeto no da la respuesta correcta, AÑADIR: Beatriz continúa diciendo: «Si quieres que algo se haga, debes hacerlo tú misma».

PREGUNTA: ¿Qué quiere Beatriz que haga Miguel?

A.2.8.2. Respuesta literal. Respuesta criterio: Beatriz quiere que Miguel coloque las estanterías. Esta respuesta puntuaría 1. Cualquier otra respuesta sería un 0.

\section{A.2.9. HISTORIA 9}

Jessica y Manuel están jugando con un juego de trenes. Jessica tiene el tren azul y Manuel el tren rojo. Jessica le dice a Manuel:

«No me gusta este tren".

PREGUNTA: ¿Qué quiere decir realmente Jessica cuando dice esto?

A.2.9.1. Respuesta literal. Respuesta criterio: Jessica quiere decir "Quiero tu tren, y tú puedes tener el mío". Esta respuesta se puntuaría con un 2 y se pasaría a la historia siguiente.

$\mathrm{Si}$ el sujeto no da la respuesta correcta, AÑADIR: Jessica continúa diciendo: «El rojo es mi color favorito".

PREGUNTA: ¿Qué quiere Jessica que haga Manuel?

A.2.9.2. Respuesta literal. Respuesta criterio: Jessica quiere intercambiar los trenes. Esta respuesta puntuaría 1. Cualquier otra respuesta sería un 0.

\section{A.2.10. HISTORIA 10}

Patricia acaba de bajar del tren con tres pesadas maletas. Juan está detrás de ella. Patricia le dice a Juan:

"¡Caramba!, estas maletas son una molestia".

PREGUNTA: ¿Qué quiere decir realmente Patricia cuando dice esto?

A.2.10.1. Respuesta literal. Respuesta criterio: Patricia quiere decir "¿Podrías ayudarme con mi equipaje, por favor?». Esta respuesta se puntuaría con un 2 y se finalizaría la prueba.

Si el sujeto no da la respuesta correcta, AÑADIR: Patricia continúa diciendo: «No sé si podré arreglarme con las tres".

PREGUNTA: ¿Qué quiere Patricia que haga Juan?

A.2.10.2. Respuesta literal. Respuesta criterio: Marisa quiere que Juan le ayude con las maletas. Esta respuesta puntuaría 1. Cualquier otra respuesta sería un 0. 


\section{PERE - Prueba de Evaluación de Reconocimiento de Emociones}

Gil-Sanz et al. (2017)

\begin{tabular}{|c|c|c|c|c|c|c|c|}
\hline 1 & Alegría & Tristeza & Enfado & Sorpresa & Miedo & Asco & Neutra \\
\hline 2 & Alegría & Tristeza & Enfado & Sorpresa & Miedo & Asco & Neutra \\
\hline 3 & Alegría & Tristeza & Enfado & Sorpresa & Miedo & Asco & Neutra \\
\hline 4 & Alegría & Tristeza & Enfado & Sorpresa & Miedo & Asco & Neutra \\
\hline 5 & Alegría & Tristeza & Enfado & Sorpresa & Miedo & Asco & Neutra \\
\hline 6 & Alegría & Tristeza & Enfado & Sorpresa & Miedo & Asco & Neutra \\
\hline 7 & Alegría & Tristeza & Enfado & Sorpresa & Miedo & Asco & Neutra \\
\hline 8 & Alegría & Tristeza & Enfado & Sorpresa & Miedo & Asco & Neutra \\
\hline 9 & Alegría & Tristeza & Enfado & Sorpresa & Miedo & Asco & Neutra \\
\hline 10 & Alegría & Tristeza & Enfado & Sorpresa & Miedo & Asco & Neutra \\
\hline 11 & Alegría & Tristeza & Enfado & Sorpresa & Miedo & Asco & Neutra \\
\hline 12 & Alegría & Tristeza & Enfado & Sorpresa & Miedo & Asco & Neutra \\
\hline 13 & Alegría & Tristeza & Enfado & Sorpresa & Miedo & Asco & Neutra \\
\hline 14 & Alegría & Tristeza & Enfado & Sorpresa & Miedo & Asco & Neutra \\
\hline 15 & Alegría & Tristeza & Enfado & Sorpresa & Miedo & Asco & Neutra \\
\hline 16 & Alegría & Tristeza & Enfado & Sorpresa & Miedo & Asco & Neutra \\
\hline 17 & Alegría & Tristeza & Enfado & Sorpresa & Miedo & Asco & Neutra \\
\hline 18 & Alegría & Tristeza & Enfado & Sorpresa & Miedo & Asco & Neutra \\
\hline 19 & Alegría & Tristeza & Enfado & Sorpresa & Miedo & Asco & Neutra \\
\hline 20 & Alegría & Tristeza & Enfado & Sorpresa & Miedo & Asco & Neutra \\
\hline 21 & Alegría & Tristeza & Enfado & Sorpresa & Miedo & Asco & Neutra \\
\hline 22 & Alegría & Tristeza & Enfado & Sorpresa & Miedo & Asco & Neutra \\
\hline 23 & Alegría & Tristeza & Enfado & Sorpresa & Miedo & Asco & Neutra \\
\hline 24 & Alegría & Tristeza & Enfado & Sorpresa & Miedo & Asco & Neutra \\
\hline 25 & Alegría & Tristeza & Enfado & Sorpresa & Miedo & Asco & Neutra \\
\hline 26 & Alegría & Tristeza & Enfado & Sorpresa & Miedo & Asco & Neutra \\
\hline 27 & Alegría & Tristeza & Enfado & Sorpresa & Miedo & Asco & Neutra \\
\hline 28 & Alegría & Tristeza & Enfado & Sorpresa & Miedo & Asco & Neutra \\
\hline 29 & Alegría & Tristeza & Enfado & Sorpresa & Miedo & Asco & Neutra \\
\hline
\end{tabular}




\begin{tabular}{|c|c|c|c|c|c|c|c|}
\hline 30 & Alegría & Tristeza & Enfado & Sorpresa & Miedo & Asco & Neutra \\
\hline 31 & Alegría & Tristeza & Enfado & Sorpresa & Miedo & Asco & Neutra \\
\hline 32 & Alegría & Tristeza & Enfado & Sorpresa & Miedo & Asco & Neutra \\
\hline 33 & Alegría & Tristeza & Enfado & Sorpresa & Miedo & Asco & Neutra \\
\hline 34 & Alegría & Tristeza & Enfado & Sorpresa & Miedo & Asco & Neutra \\
\hline 35 & Alegría & Tristeza & Enfado & Sorpresa & Miedo & Asco & Neutra \\
\hline 36 & Alegría & Tristeza & Enfado & Sorpresa & Miedo & Asco & Neutra \\
\hline 37 & Alegría & Tristeza & Enfado & Sorpresa & Miedo & Asco & Neutra \\
\hline 38 & Alegría & Tristeza & Enfado & Sorpresa & Miedo & Asco & Neutra \\
\hline 39 & Alegría & Tristeza & Enfado & Sorpresa & Miedo & Asco & Neutra \\
\hline 40 & Alegría & Tristeza & Enfado & Sorpresa & Miedo & Asco & Neutra \\
\hline 41 & Alegría & Tristeza & Enfado & Sorpresa & Miedo & Asco & Neutra \\
\hline 42 & Alegría & Tristeza & Enfado & Sorpresa & Miedo & Asco & Neutra \\
\hline 43 & Alegría & Tristeza & Enfado & Sorpresa & Miedo & Asco & Neutra \\
\hline 44 & Alegría & Tristeza & Enfado & Sorpresa & Miedo & Asco & Neutra \\
\hline 45 & Alegría & Tristeza & Enfado & Sorpresa & Miedo & Asco & Neutra \\
\hline 46 & Alegría & Tristeza & Enfado & Sorpresa & Miedo & Asco & Neutra \\
\hline 47 & Alegría & Tristeza & Enfado & Sorpresa & Miedo & Asco & Neutra \\
\hline 48 & Alegría & Tristeza & Enfado & Sorpresa & Miedo & Asco & Neutra \\
\hline 49 & Alegría & Tristeza & Enfado & Sorpresa & Miedo & Asco & Neutra \\
\hline 50 & Alegría & Tristeza & Enfado & Sorpresa & Miedo & Asco & Neutra \\
\hline 51 & Alegría & Tristeza & Enfado & Sorpresa & Miedo & Asco & Neutra \\
\hline 52 & Alegría & Tristeza & Enfado & Sorpresa & Miedo & Asco & Neutra \\
\hline 53 & Alegría & Tristeza & Enfado & Sorpresa & Miedo & Asco & Neutra \\
\hline 54 & Alegría & Tristeza & Enfado & Sorpresa & Miedo & Asco & Neutra \\
\hline 55 & Alegría & Tristeza & Enfado & Sorpresa & Miedo & Asco & Neutra \\
\hline 56 & Alegría & Tristeza & Enfado & Sorpresa & Miedo & Asco & Neutra \\
\hline
\end{tabular}

A continuación, se muestra un ejemplo de la aplicación de la prueba: 


\section{Prueba de Evaluación del Reconocimiento de Emociones (PERE)}

A continuación verás 56 fotografías

Debes indicar la emoción que crees que representan 


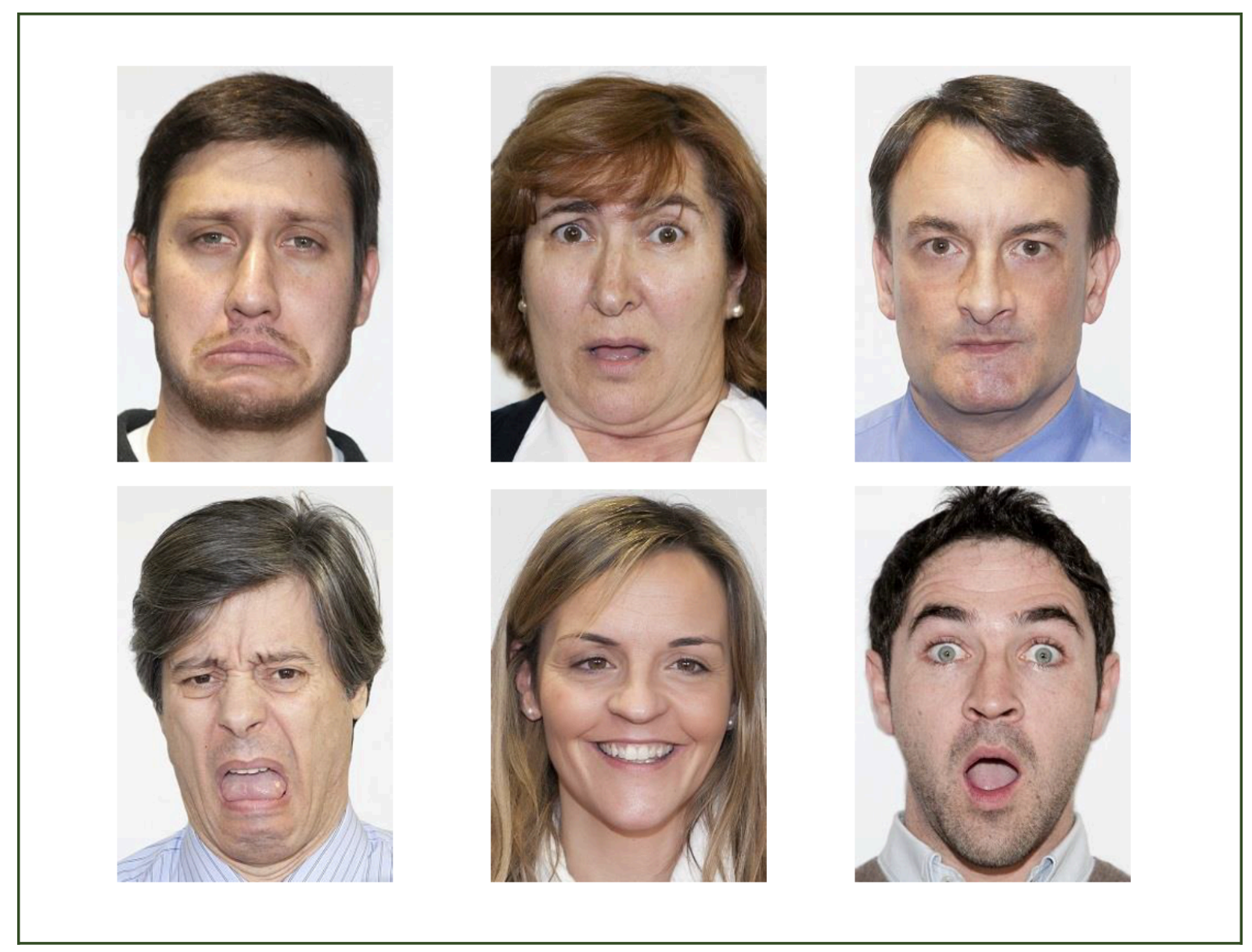


Ejemplo de la versión computarizada de la prueba Beads Task (Dudley y Over, 2003).

\section{Tenemos dos tarros. En el tarro principalmente verde hay 60 bolas verdes y 40 bolas moradas. En el tarro principalmente morado hay 60 bolas moradas y 40 bolas verdes.}

Pulsa la barra espaciadora para continuar 


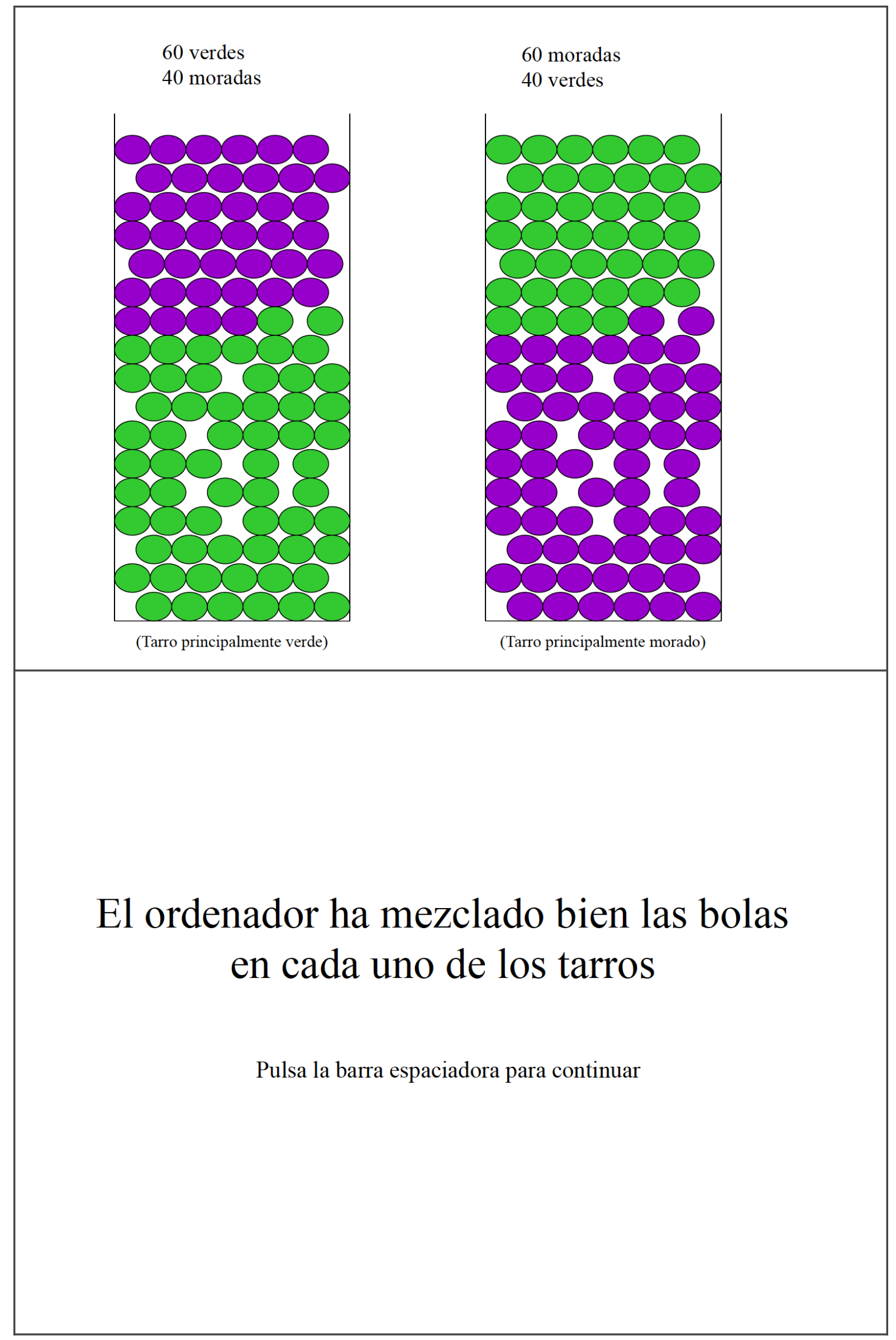




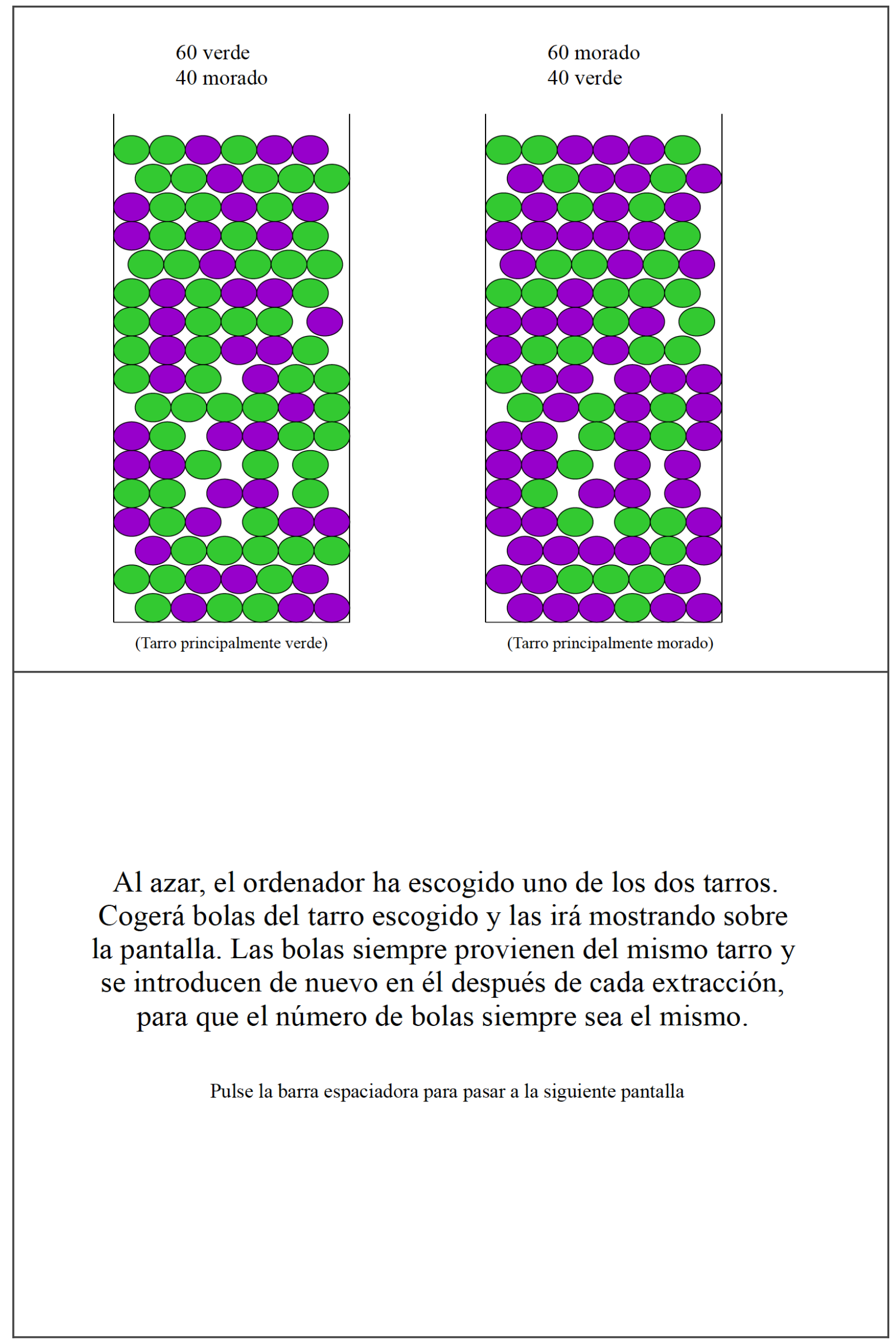


Su tarea consiste en decidir de cuál de los tarros son las bolas. Para ello, puedes ver tantas bolas como quieras antes de tomar la decisión. Por lo tanto, después de que aparezca la primera bola, puede pedir otra o decirme si sabe de qué tarro proviene: el tarro principalmente verde o el tarro principalmente morado.

\section{Pulse la barra espaciadora para continuar}

Ahora verá la primera bola.

Recuerde que puede ver tantas bolas como quiera antes de tomar la decisión sobre el tarro del que provienen.

Tome la decisión cuando esté seguro.

Pulse la barra espaciadora para continuar 
La primera bola es

Pulse barra espaciadora para continuar

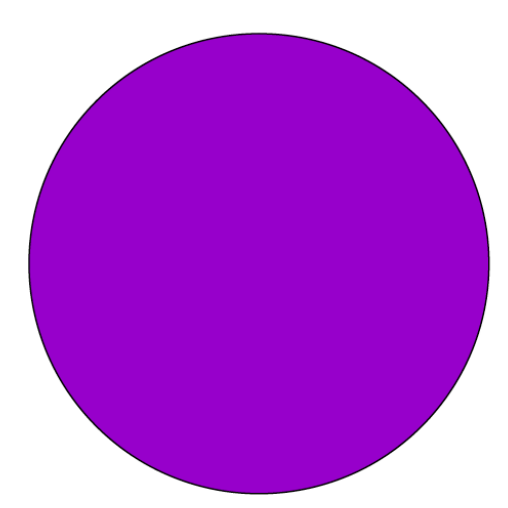

BOLAS PREVIAMENTE VISTAS

- Y así sucesivamente hasta que el/la paciente tome una decisión.

CORRECCIÓN Y PUNTUACIÓN:

- Se anotará el número de bolas que el/la paciente ha necesitado para llegar a una conclusión.

- Si ha tomado la decisión tras la primera o segunda bola se considerará que exhibe un "sesgo de saltar a conclusiones".

- № de bolas:

- Salto a conclusiones: SI $\square$ NO $\square$

- Opcionalmente se le puede preguntar a la persona evaluada qué nivel de confianza tiene sobre esa conclusión (en \%).

- Nivel de certeza: $\square \%$ 


\section{SHP-S}

Datos de identificación

\section{Nombre del evaluado: \\ Nombre del examinador:}

Edad:

Sexo:

Nivel educativo:

Motivo de consulta:

Resumen de resultados

Baremo utilizado:

\section{Forma 1}

Fecha de evaluación:

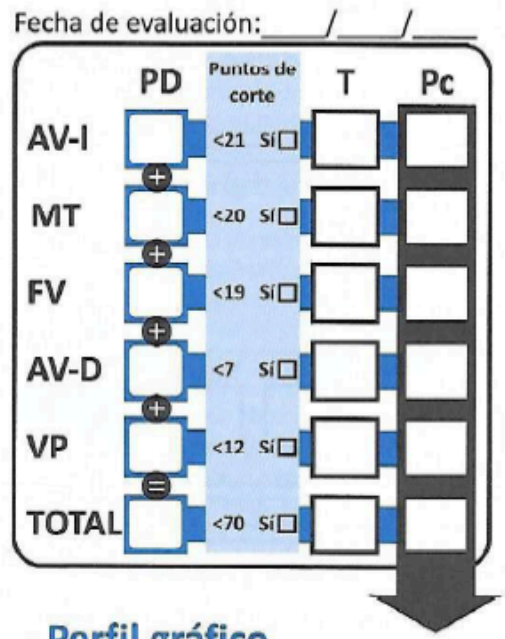

\section{Forma 2}

Fecha de evaluación:

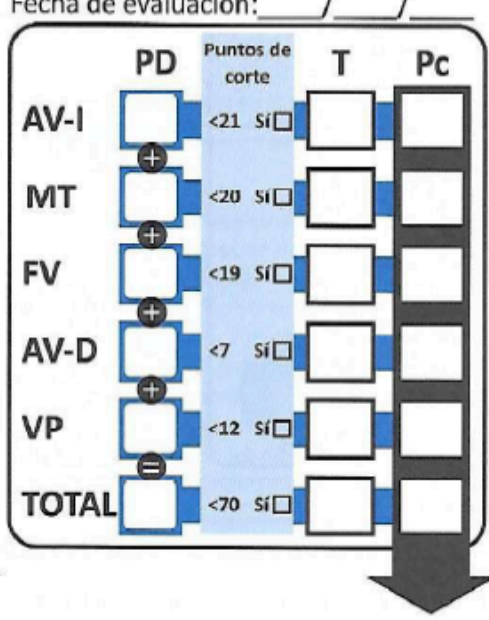

\section{Forma 3}

Fecha de evaluación:

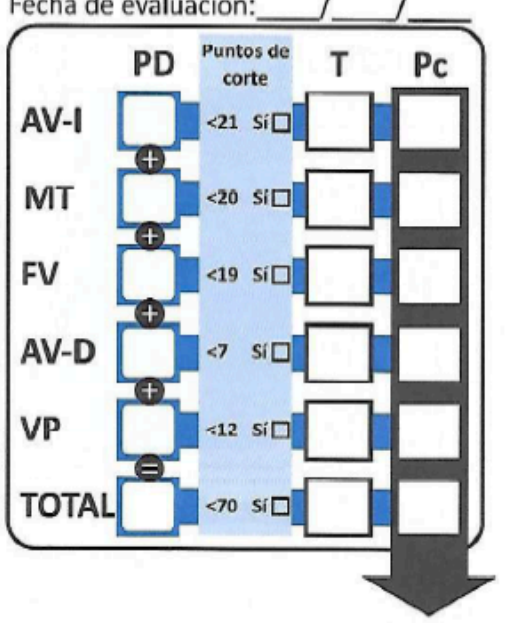

Perfil gráfico

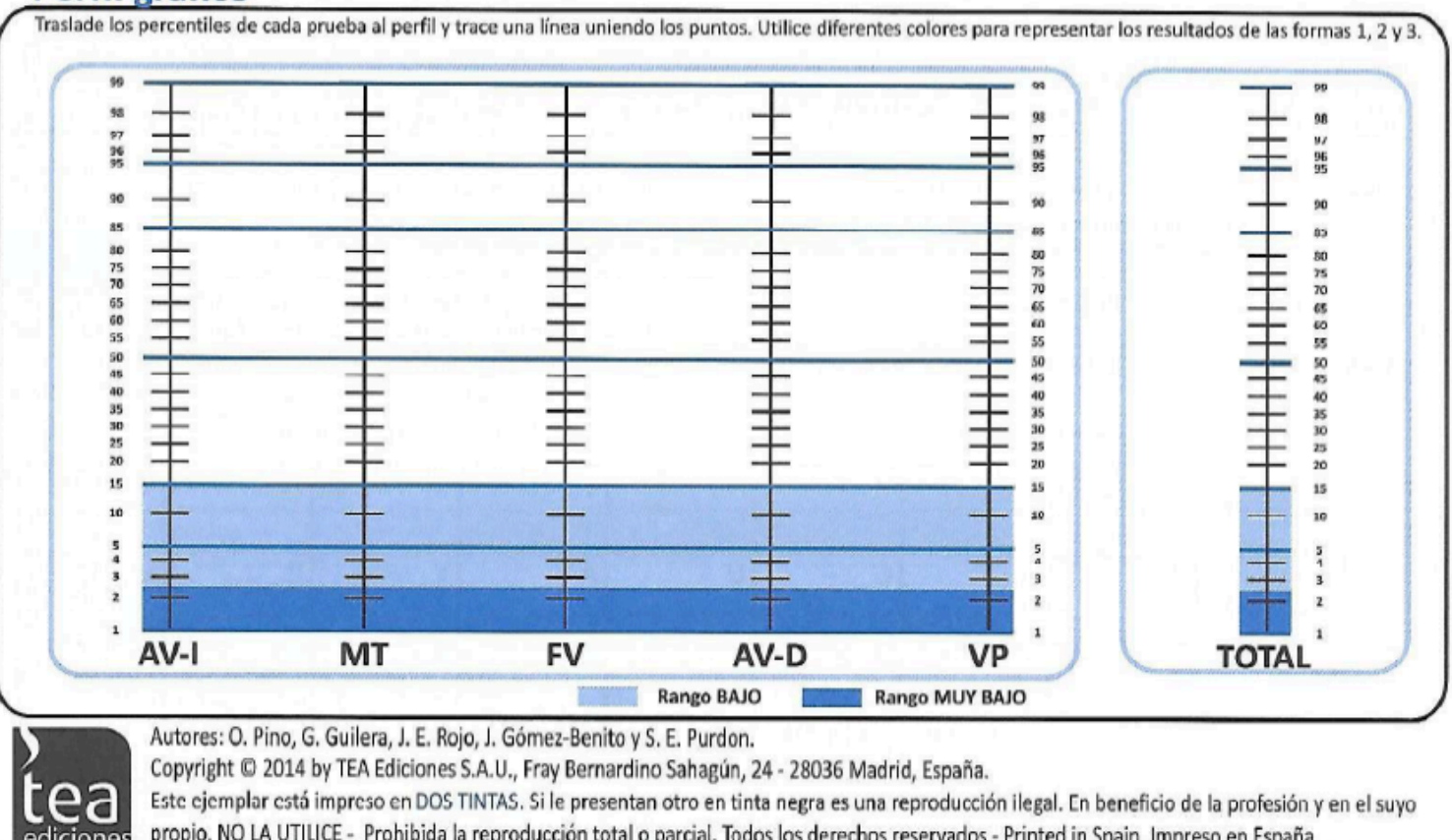




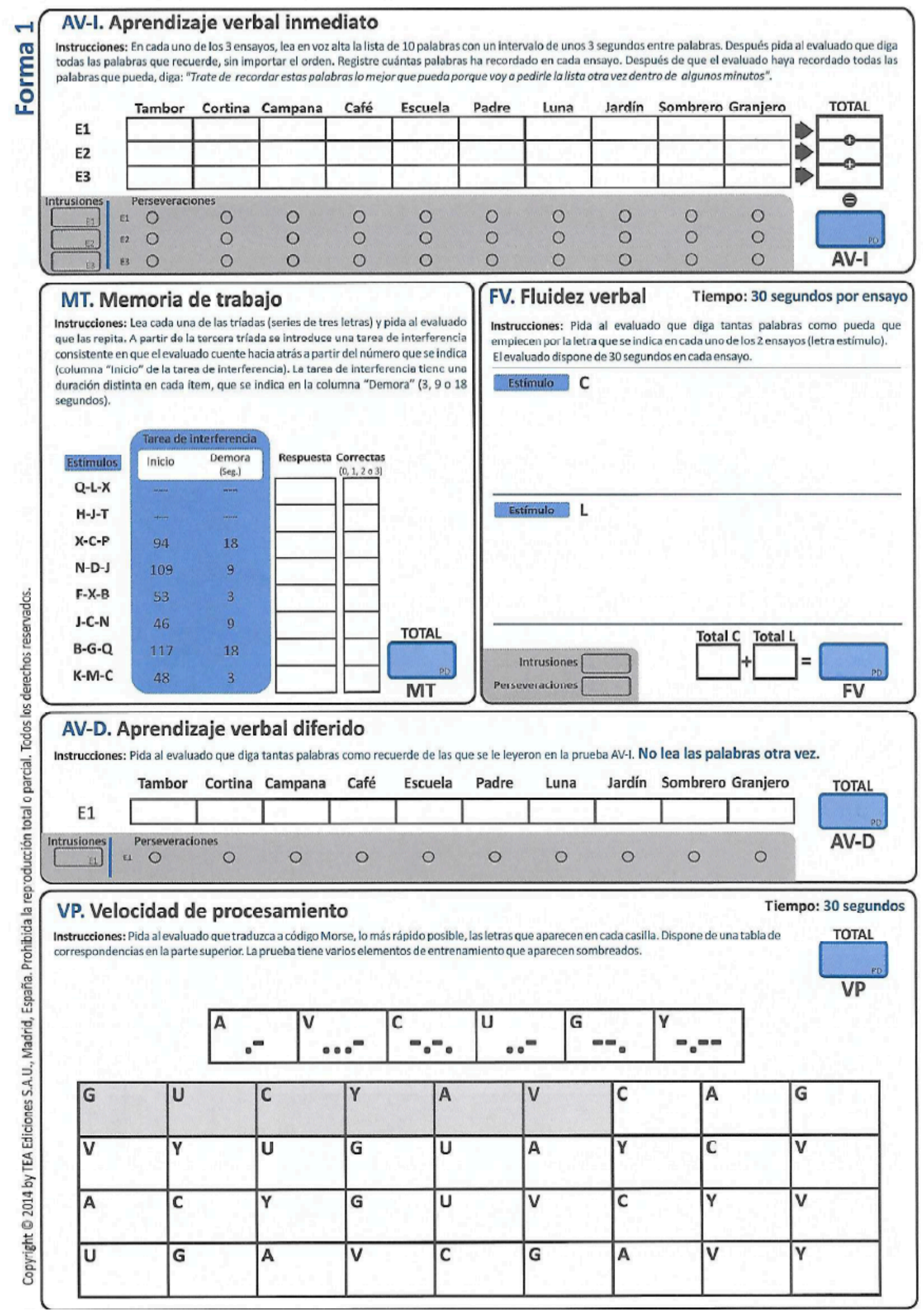




\section{AV-I. Aprendizaje verbal inmediato}

Instrucciones: En cada uno de los 3 ensayos, lea en voz alta la lista de 10 palabras con un intervalo de unos 3 segundos por palabra. Después pida al evaluado que diga todas las palabras que recuerde, sin importar el orden. Registre cuántas palabras ha recordado en cada ensayo.

- Ensayo 1: "Voy a leerle una lista de palabras. A continuación, quisiera que usted me repitiera tantos palabras como recuerde, en cualquier orden".

- Ensayo 2: "Ahora voy a leer la lista de palabras otra vez. Cuando acabe, repita de nuevo tantas palabras como pueda recordar en cualquier orden, incluyendo las que usted ya me ha dicho antes".

- Ensayo 3: "Voy a repetir la lista una vez más. Como antes, cuando acabe, repitame tantas palabras como recuerde, en cualquier ordcn, incluyendo las que usted ya me habia dicho".

Después de que el evaluado haya recordado todas las palabras que pueda, diga: "Trate de recordar estas palabras lo mejor que pueda porque voy a pedirle la lista otra vez dentro de alqunos minutos".

Puntuación: Sume el número total de palabras recordadas en cada ensayo y anótelo en la casilla AV-I.

\section{MT. Memoria de trabajo}

Instrucciones: Lea cada una de las tríadas (serie de tres letras) y pida al evaluado que las repita. A partir de la tercera tríada se introduce una tarea de interferencia consictẹntẹ ę que el evaluado cuente hacia atrás a partir del númere que se indica (columna "Inicie" de la tarea de interferencia). La tarea de interferencia tiene una duración distinta en cada item, que se indica en la columna "Demora" (3, 9018 segundos).

- items 1 y 2: "Le voy a decir algunas letras y cuando haya acabado me gustaria que usted me las repitiera. ¿De acuerdo?".

- ítems 3 a 8: "Ahora voy a decirle algunas letras y un número. Esta vez quisiera que usted contara hacia atrás desde el número hasta que le pregunte por las letras. Por ejemplo, si digo ABC-10 usted contaría hacia atrás en voz alta a partir del 10 hasta que le pregunte por las letras, $y$ entonces usted me diria $A B C$ ".

Puntuación: Registre el número de letras recordadas correctamente en cada tríada $(0,1,203)$. No importa el orden en que las haya dicho. Sume el numero total de letras recordadas en todos los items. Anote el resultado en la casilla MI.

$\begin{array}{ll}\text { FV. Fluidez verbal } & \text { Tiempo: } 30 \text { segundos por ensayo }\end{array}$

Instrucciones: Pida al evaluado que diga tantas palabras como pueda que empiecen por una letra en particular. Se aplican 2 ensayos con letras diferentes (letra estímulo) que se indican en cada caso. El evaluado dispone de 30 segundos en cada ensayo.

- Ensayo 1: "Voy a decir una letra del alfabeto y quisiera que usted me dijera tantas palabras como pueda que comiencen con eso letra. Hay tres normas en esta tarea. La primera regla es que usted no puede utilizar palabras que sean números, por ejemplo, si le dijera la letra "T" no puede decir treinta y uno o treinta y dos. La segunda norma es que usted no puede utilizar ninguna palabra que sea un nombre propio, por ejemplo, el nombre de un lugar, de una persona o de una ciudad. La tercera regla es que usted no debe decir una palabra y realizar derivados, por ejemplo, baile, bailar y bailarín. ¿Tiene alguna duda? La primera letra es _. Ahora digame todas las palabras que pueda que empiecen con la letra ".".

- Ensayo 2: "Ahora digame todas los palabras que pueda que empiecen con la letra _".

Puntuación: Cuente el número de palabras correctamente evocadas en los dos ensayos. No se consideran correctas las repeticiones ni las palabras que Incumplan las normas fijadas (ver manual). Anote el resultado en la casilla FV.

\section{AV-D. Aprendizaje verbal diferido}

Instrucciones: Pida al evaluado que diga tantas palabras como recuerde de las que se le leyeron en la prueba AV-I. No lea las palabras otra vez. - Ensayo 1: "¿Recuerda esa lista de pulubrus yue unteriumente dijimus tres veces? Quisiera que me diljera tantas palabras como recuerde de esa lista, en cualquier orden".

Puntuación: Cuente el número de palabras correctamente recordadas. Anote el resultado en la casilla AV-D.

\section{VP. Velocidad de procesamiento}

Tiempo: 30 segundos

Instrucciones: Pida al evaluado que traduzca a código Morse, lo más rápido posible, las letras que aparecen en cada casilla. Dispone de una tabla de correspondencias en la parte superior. La prueba tiene varios elementos de entrenamiento que aparecen sombreados. El evaluado dispone de 30 segundos para realizar la tarea. El evaluado realiza la tarea en este ejemplar.

- Entrenamiento: "Si usted mira estas letras (señale la tabla de correspondencias) verá que cada letra tiene un código. En la parte de abajo lo que dehe hacer es rnmpletar mala casilla con el código que corresponde a cada letra". Complete tres de las letras verbalizando axplícitamente el proceso, diciendo "Esto es una __ así que pondré __.". Después de completar las tres primeras, diga: "¿l.o entiende? Ahora me gustaria que usted hiciera estos tres ejemplos restantes del área sombreada para practicar", corrigiendo cualquier error del evaluado.

- Prueba: "Cuando le avise, intente rellenar estos (señalando), uno detrás de otro y lo mós rápido que pueda, sin cometer errores. ¿Tiene alguna pregunta? Empiece ya". Comience a cronometrar.

Puntuación: Cuente el número de letras correctamente codificadas. Anote el resultado en la casilla VP.

\section{Transformación de las puntuaciones}

Para obtener las puntuaciones transformadas de cada una de las pruebas siga los siguientes pasos:

1. Obtenga las puntuaciones directas (PD) de cada prueba y traslade estos valores a las casillas correspondientes a la forma aplicada de la tabla dc resultados de la portada del ejemplar.

2. Calcule la puntuación directa total del SCIP-S sumando las puntuaciones directas de las cinco pruebas.

3. Seleccione en el manual el baremo adecuado en función del tipo de comparación que desea realizar (baremos clínicos o baremos para población general) así como de la edad y del nivel educativo.

4. Localice la PD de cada prueba en la tabla y anote el percentil $(\mathrm{PC})$ y la puntuación T correspondiente en la portada del ejemplar.

5. Traslade los percentiles obtenidos en el paso anterior al perfil gráfico de resultados de la portada marcándolos con un punto.

6. Trace una linea uniendo los puntos marcados para obtener el perfil gráfico de las puntuaciones. Si se evalúa a la misma persona en varias

ocasiones utilizando las distintas formas paralelas es recomendable utilizar diferentes colores para representar el perfil de cada evaluación.

Copyright (C) 2014 by TEA Ediciones S.A.U., Madrid, España. Prohibida la reproducción total o parcial. Todos los derechos reservados. 
